\title{
Towards clinical applications in fMRI neurofeedback : learning how to change brain states
}

Citation for published version (APA):

Zilverstand, A. (2014). Towards clinical applications in fMRI neurofeedback : learning how to change brain states. [Doctoral Thesis, Maastricht University]. Maastricht University. https://doi.org/10.26481/dis.20140702az

Document status and date:

Published: 01/01/2014

DOI:

10.26481/dis.20140702az

Document Version:

Publisher's PDF, also known as Version of record

\section{Please check the document version of this publication:}

- A submitted manuscript is the version of the article upon submission and before peer-review. There can be important differences between the submitted version and the official published version of record.

People interested in the research are advised to contact the author for the final version of the publication, or visit the DOI to the publisher's website.

- The final author version and the galley proof are versions of the publication after peer review.

- The final published version features the final layout of the paper including the volume, issue and page numbers.

Link to publication

\footnotetext{
General rights rights.

- You may freely distribute the URL identifying the publication in the public portal. please follow below link for the End User Agreement:

www.umlib.nl/taverne-license

Take down policy

If you believe that this document breaches copyright please contact us at:

repository@maastrichtuniversity.nl

providing details and we will investigate your claim.
}

Copyright and moral rights for the publications made accessible in the public portal are retained by the authors and/or other copyright owners and it is a condition of accessing publications that users recognise and abide by the legal requirements associated with these

- Users may download and print one copy of any publication from the public portal for the purpose of private study or research.

- You may not further distribute the material or use it for any profit-making activity or commercial gain

If the publication is distributed under the terms of Article $25 \mathrm{fa}$ of the Dutch Copyright Act, indicated by the "Taverne" license above, 
Towards clinical applications in fMRI neurofeedback.

Learning how to change brain states.

Anna Zilverstand 
(C) Anna Zilverstand, Maastricht 2014

All rights reserved. No part of this thesis may be reproduced, stored in a retrieval system, or transmitted in any form, or by any means, electronic, mechanical, photocopying, recording or otherwise, without prior written permission of the author.

Cover: Photograph by cgiraldez (http://www.morguefile.com/creative/cgiraldez) Production: CPI Wöhrmann Print Services B.V.

ISBN: 978-94-6203-608-6 
Towards clinical applications in fMRI neurofeedback. Learning how to change brain states.

\section{Dissertation}

to obtain the degree of Doctor at Maastricht University, on the authority of Rector Magnificus, Prof. dr. L.L.G. Soete, in accordance with the decision of the Board of Deans, to be defended in public

on Wednesday $2^{\text {nd }}$ of July, 2014 at $12: 00$ hours

by Anna Zilverstand 


\section{Supervisor}

Prof. Dr. Rainer Goebel

\section{Co-supervisor}

Dr. Bettina Sorger

\section{Assessment Committee}

Prof. Dr. Bernadette Jansma

(Chair)

Prof. Dr. Arnoud Arntz

(University of Amsterdam)

Prof. Dr. David Linden

(Cardiff University, England)

Dr. Nikolaus Weiskopf

(University College London, England)

Dr. Vincent van de Ven

The work presented in this thesis was funded by the BrainGain Smart Mix

Programme of the Dutch Ministry of Economic Affairs and the Dutch Ministry of Education, Culture and Science (grant number: SSM06011). 


\section{Contents}

General Introduction

Chapter 1 Where are we in fMRI neurofeedback therapy:

Real-time fMRI-based brain-computer-interfacing for neurofeedback therapy and compensation of lost motor functions.

Chapter 2 New tools for fMRI neurofeedback:

Windowed correlation: a suitable tool for providing dynamic fMRI-based functional connectivity neurofeedback on task difficulty.

Chapter 3 Measuring anxiety based on the brain:

Quantitative representations of subjective anxiety levels in the brain. A parametric fMRI study in spider phobia.

Chapter 4 fMRI neurofeedback for regulating anxiety:

fMRI neurofeedback enhances efficacy of cognitive reappraisal for anxiety regulation in spider phobia.

Chapter 5 fMRI neurofeedback in ADHD patients:

fMRI-based neurofeedback from anterior cingulate cortex for adults with Attention-Deficit/Hyperactivity Disorder. A proof of concept study.

Summary and Conclusions

Acknowledgments

Curriculum Vitae

Publications 

small interventions can have large effects

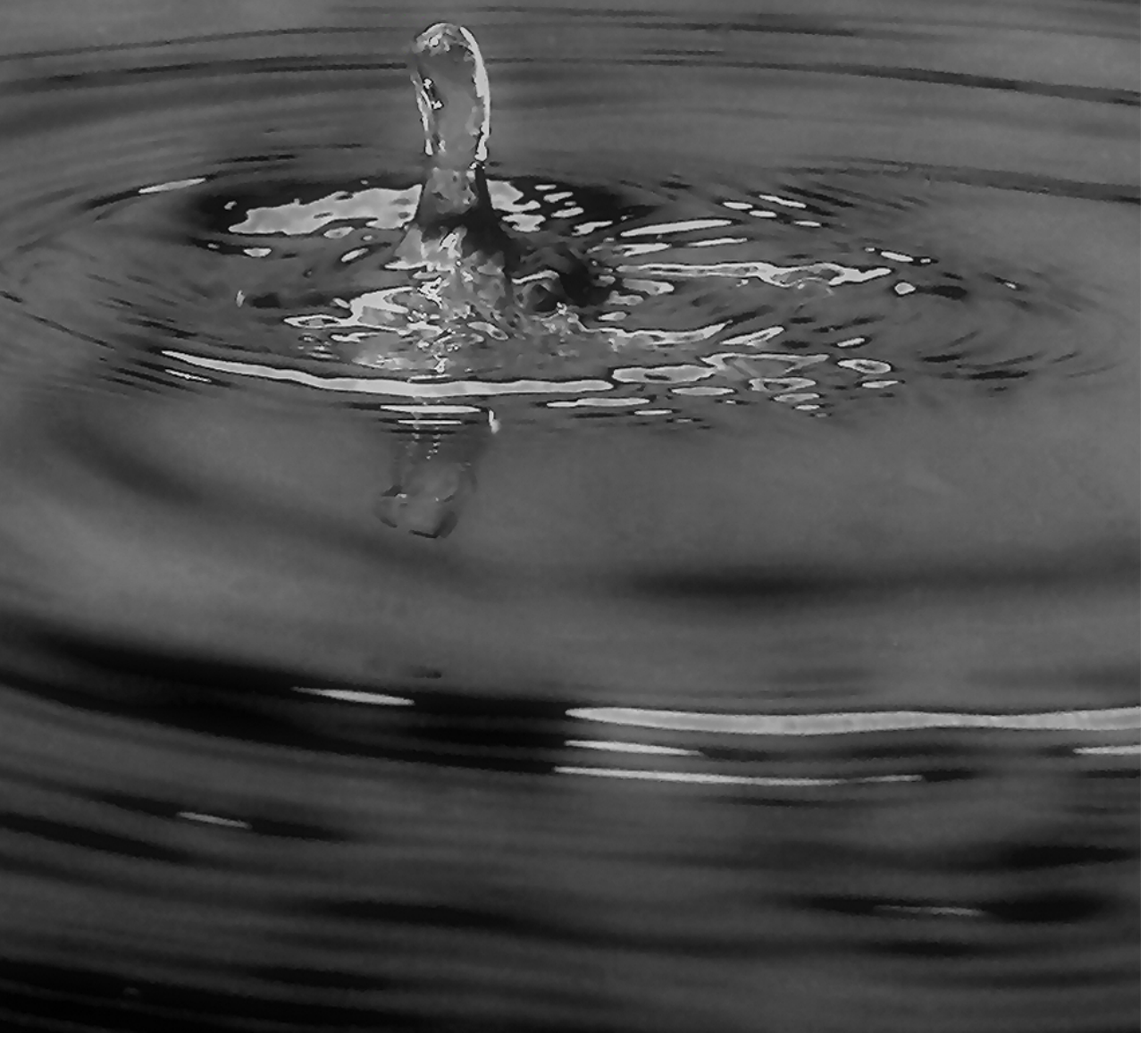



Introduction 


\section{History of mental health treatment}

The idea that a mad person deserves charity, instead of being left to suffer from a message sent by God or astrology, originated in the $18^{\text {th }}$ century (figure 1). For the first time madness was seen as something that could be treated by gentle discipline, order, and well intentioned manipulation, rather than being a defect of the soul that was irreparable (English Heritage Foundation, 2012). Private asylums for mentally ill became business, and support for people with disabilities a Christians' duty (figure 2). Slowly, the notion that an institution is the 'right place' for people who are 'different' became commonplace. A major factor for bringing mental illness to public attention in England was the ill health of George III (1738-1820), who suffered recurrent periods of mania that his physicians were unable to control (Bewley, 2008). The change in

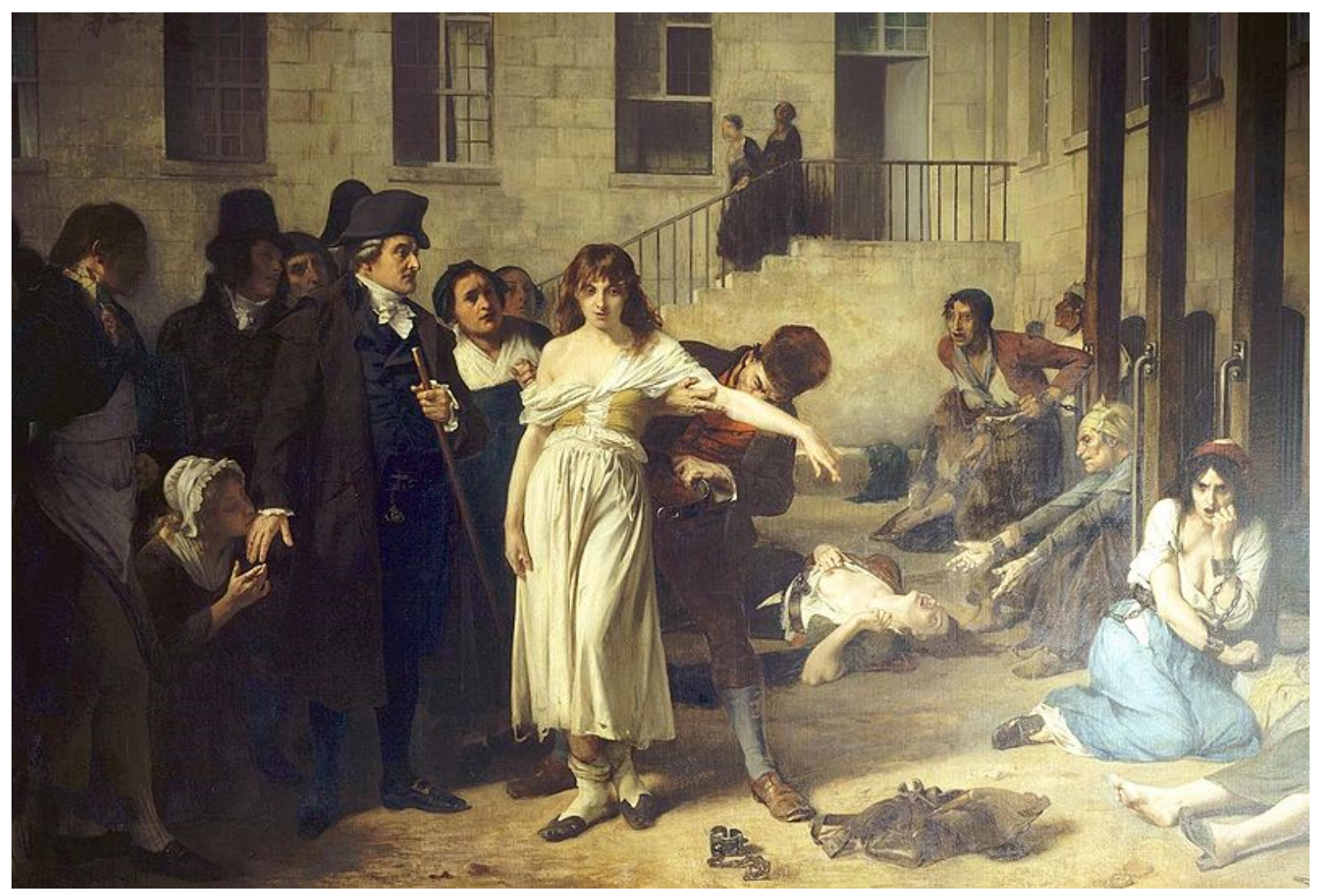

Figure 1. Dr. Pinel at Salpêtrière mental hospital, by Tony Robert-Fleury (1795). "During the Enlightenment attitudes towards the mentally ill began to change, it came to be viewed as a disorder that required compassionate treatment. In the painting Dr. Pinel orders the removal of chains from patients at the Paris Asylum for insane women." ("History of psychiatric institutions," 2014) 
societal context also arouse interest of the medical profession in diagnostics, therapeutic and legal aspects of the care of the insane. In 1808, a bill for 'better care and maintenance of lunatics', was passed in England in an attempt to regulate spreading abuse in private institutions (Bewley, 2008). For the first time comprehensive public financial support for the opening of 'asylums' was available.

By the end of the $19^{\text {th }}$ century, many countries in Europe, and the United States had established a national system for regulating the rapidly growing mental hospitals (Shorter, 1997b). The number of people living in English psychiatric institutions had increased from a few thousand to about 100,000 'idiots and lunatics' (English Heritage Foundation, 2012), and a new class of medical professionals, the 'alienists' (later

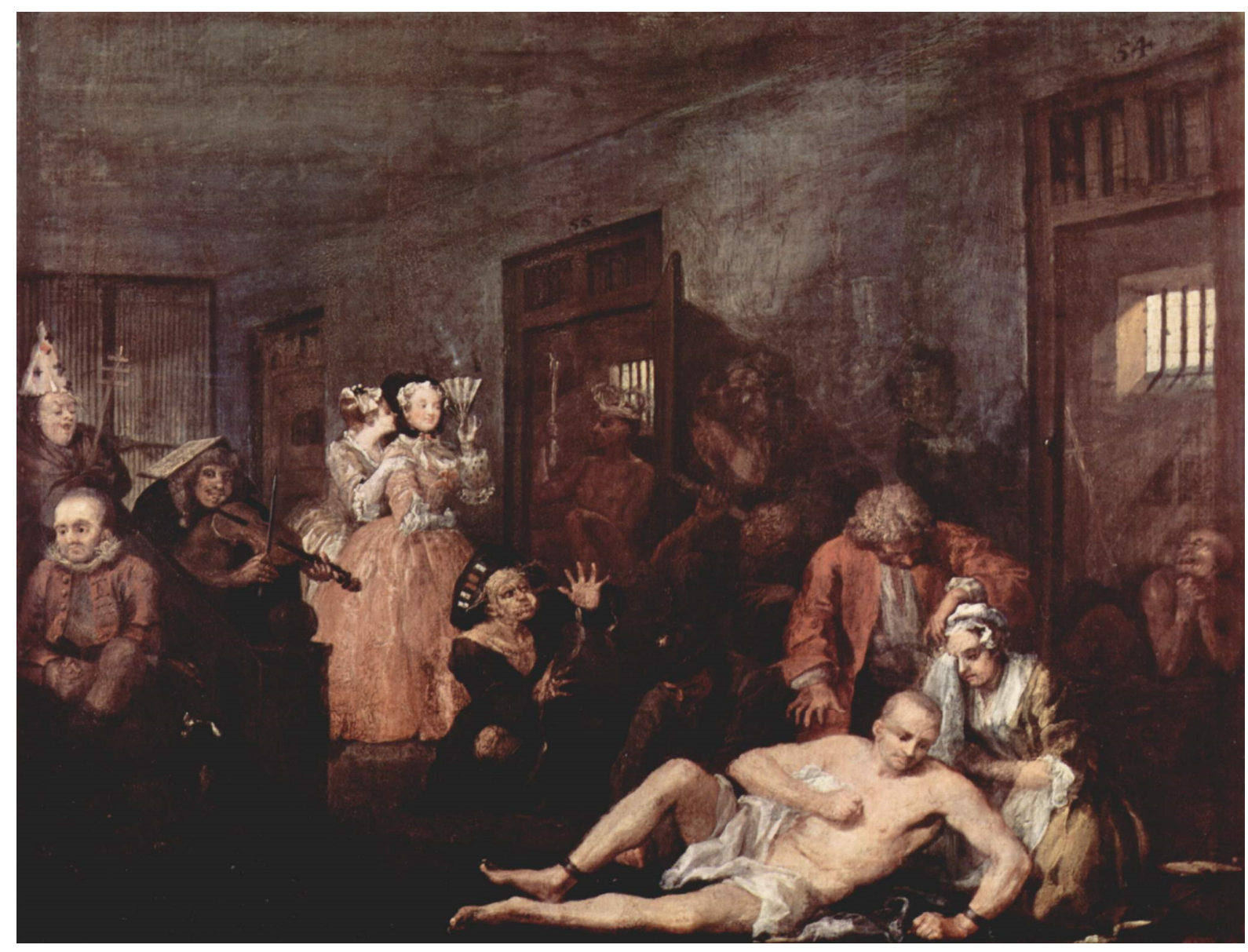

Figure 2. Bethlehem Royal Hospital for mental illness, by William Hogarth (1733). "The painting shows the fall of Tom Rakewell, son of a rich merchant, who ends his days insane and violent in Bethlehem Hospital, London's celebrated mental asylum. Only his faithful wife Sarah Young is there to comfort him. The fashionably dressed women in the background have come to the asylum as a social occasion, to be entertained by the bizarre antics of the inmates." ("Rake's Progress," 2014) 
known as a 'psychiatrists') had formed. Physical activity, quietness, and 'moral treatment' were considered the main ingredients of therapy, and well-off patients were assembled to exercise while the poor worked under harsh regimes for their living (English Heritage Foundation, 2012). However, at the end of the $19^{\text {th }}$ century, after a hundred years of growing hospitalization, the hope that mental illness could be easily ameliorated through exercise and morality seemed disappointed, and patients were considered 'incurable' by many (Shorter, 1997b). The 'therapeutic optimism' of the beginning of the century seemed lost. Around this time, Sigmund Freud started his medical career in the Vienna General Hospital, where he worked in the 'lunatic asylum' for a short time. Freud started collecting detailed descriptions of clinical pictures, and worked on developing psychoanalysis as a treatment in his private practice. After a culture of therapeutic nihilism, his approach gave hope to physicians all over the world, coming to dominate the field, and establishing a psychological approach to treatment for the first time (Mayou, 1989).

In general the beginning of the $20^{\text {th }}$ century was a time of radical and experimental treatments. Schizophrenia was being treated by inducing insulin comas, an unpleasant and dangerous procedure, which supposedly stimulated the automatic nervous system, and only lead to improvements in some cases (Jones, 2000; Norman, 1938). Barbiturates were given to psychiatric patients to induce day-long deep sleep as one of the first experimental psychopharmacological treatment (Shorter, 1997a). However, mixed results and casual fatalities rapidly dampened first enthusiasm. Lobotomy, a surgical procedure for severing connections to and from the prefrontal cortex, became a popular treatment after initial reports of successes (Mashour, Walker, \& Martuza, 2005; Shorter, 1997a). Unfortunately, follow up studies showed that the side effects were often more debilitating than the initial disease (Mashour et al., 2005). Another treatment invented in the first half of the $20^{\text {th }}$ century was electroconvulsive shock therapy, which induces temporary seizures electrically, and is still considered an effective short-term treatment of severe depression today (Shorter \& Healy, 2007; UK ECT Review Group, 2003). Also, shortly after the first recordings of the human brain's electric potentials with electroencephalography in 1924 (Berger, 1933; Jung \& Berger, 1979), researchers realized that it was possible to reinforce rhythmic brain activation pattern characteristic for sleep, and that this affected the alertness of the experimental participants (Jasper \& Shagass, 1941; Knott \& Henry, 1941). It was later shown that repeated reinforcement of these brain rhythms 


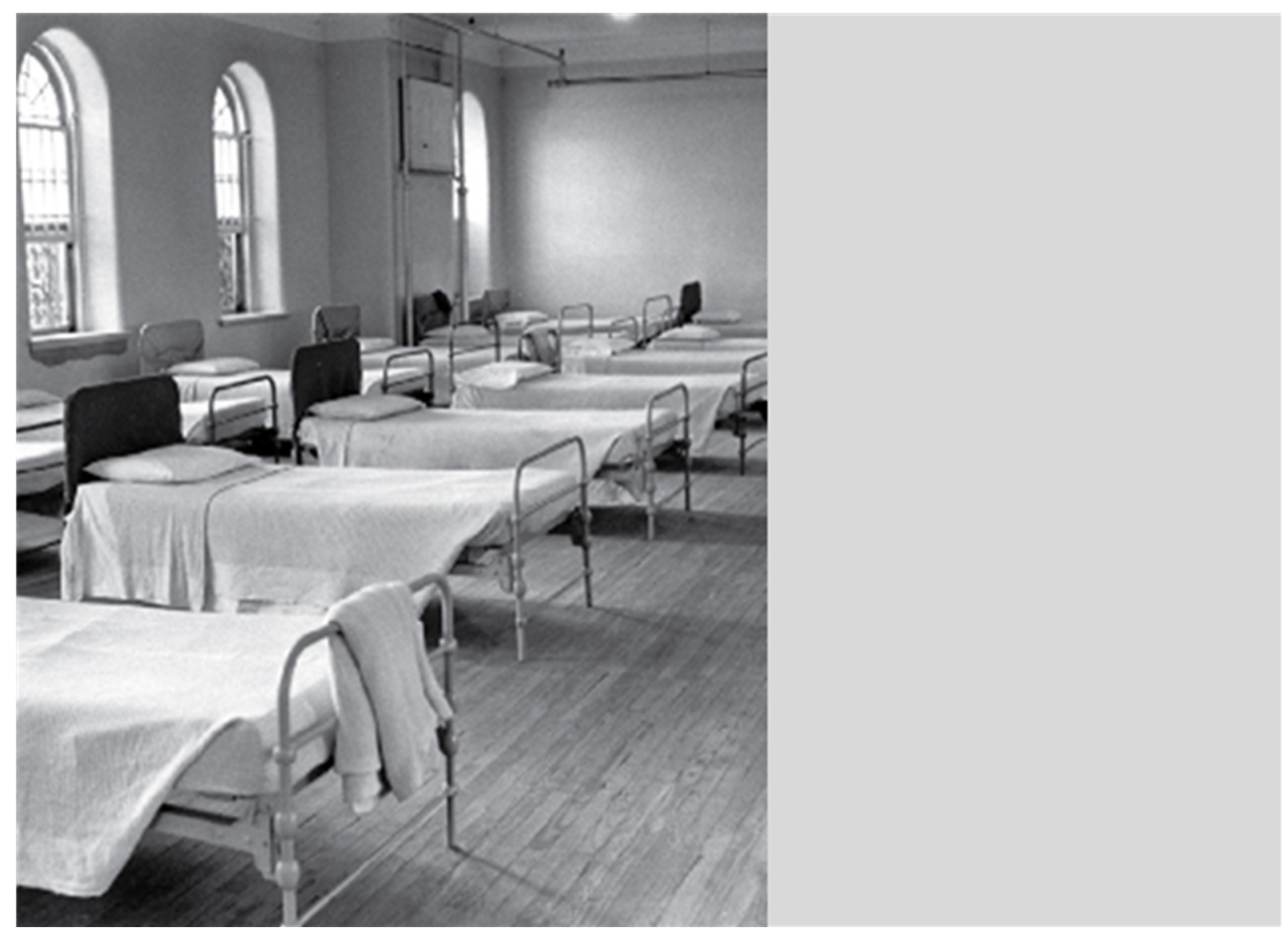

Figure 3. Dormitory in New York State Psychiatric Hospital.

"Willard Psychiatric Hospital admitted its first patients in 1869. Many of the early residents arrived after years of incarceration and mistreatment in dismal almshouses, some after allegedly failing to improve in other state institutions. Before long, Willard grew into a sizeable village, relying heavily on unpaid patient labor to sustain its operation. Factory-sized brick buildings housed patients, while the more opulent residences were designated for doctors and other staff. In the early 20th century, the patient census rose steadily, with over-crowded wards and deteriorating conditions. By 1950, New York operated 30 state hospitals with more than 120,000 patients. Willard Psychiatric Hospital in New York reached an all-time high with 4076 patients in 1955. ... By 1974, Willard's census had declined to less than a thousand, and the patient population dwindled to a few hundred by the time the facility closed in 1995 . More than 50,000 patients were admitted to Willard during its 126 -year history, and nearly half of those died there." (Penney \& Stastny, 2006) 
prolonged subsequent periods of sleep in cats (Sterman, Howe, \& Macdonald, 1970), and shortened sleep onset latency in humans (Hoedlmoser et al., 2008). Another therapeutic invention of the first half of the $20^{\text {th }}$ century, which would have a dramatic impact, was the synthetizing of the first antipsychotic drug, chlorpromazine, known as 'Largactil' and 'Thorazine', in France in 1950 in a search for new sedative drugs. French, Canadian, and American psychiatrists started administering antipsychotics to schizophrenic patients and found that it had a remarkable impact. For the first time patients suffering from schizophrenia were discharged from psychiatric institutions. In the following decade, through a combination of advances in drug treatment, and greater emphasis on human rights, the residential psychiatric population in the United States dropped by 30 per cent (Lawton-Smith \& McCulloch, 2013; Thuillier, 1999) (figure 3). Drug treatment also revolutionized the handling of depression and bipolar disorder, as the severe side effects of electroconvulsive shock therapy could now be avoided in most cases (López-Muñoz \& Alamo, 2009). In general, the first decades of the $20^{\text {th }}$ century were marked by a shift towards physiological treatments, and for the first time mental health problems were seen as a biological dysfunction of the brain.

The efforts in optimization of treatment were continued in the coming decades. In the 1960s first attempts for a clinical use of 'EEG-neurofeedback', the reinforcement of selected brain activation patterns measured with electroencephalography, showed that epilepsy patients could be trained to suppress seizure activity (Sterman \& Friar, 1972). Today, this technique is still applied in epilepsy patients who do not respond to drug treatment, and has been shown to be successful in $74 \%$ of refractory epilepsy patients (Tan et al., 2009). Also, the clinical potential of using EEG-neurofeedback to treat children with attention-deficit/hyperactivity disorder has been investigated intensively, mostly with positive results (Arns, Heinrich, \& Strehl, 2014). Furthermore, advances in the 1960s were marked by an improvement of drug treatment through the invention of new agents with less side effects, and the next big revolution in the treatment of mental disorders, the development of cognitive therapy. The inventor of cognitive therapy, Aaron T. Beck, had been trained on Freud's psychodynamic theories, and embarked on a series of experiments designed to test the notion that depression was a consequence of unconscious anger (Hollon, 1998). What Beck found was that depression did not seem to be driven by suppressed anger, but that patients demonstrated typical thoughts and beliefs, which maintained their negative emotions and behavior (Beck, 1967). Most importantly, he demonstrated that changing 
their cognitions by 'cognitive therapy' improved the depression (Rush, Beck, Kovacs, \& Hollon, 1977). Today, cognitive therapy is successfully applied in depression, anxiety disorders, stress disorder, anger management, and chronic pain management (Butler, Chapman, Forman, \& Beck, 2006). Notably, this approach has not replaced prior treatments, but patients today are offered many different efficacious treatment options.

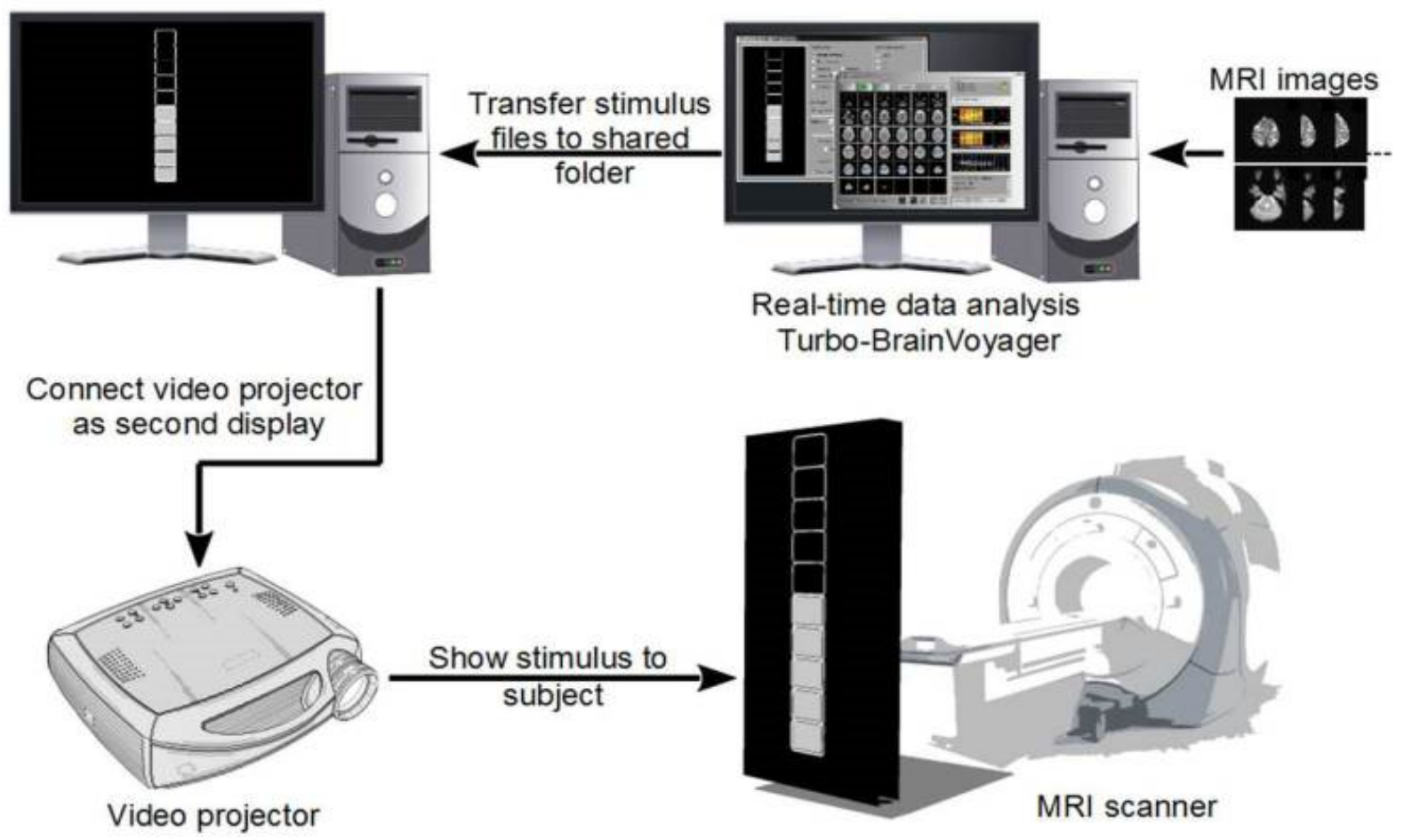

Figure 4. Exemplary setup of real-time fMRI neurofeedback experiment.

This figure illustrates the data flow during an online/real-time fMRI experiment. Following acquisition of functional data, the images are reconstructed, and processed in real-time. The analysis software transfers its output to a stimulation computer, where the feedback display is generated (here thermometer display). The feedback is then presented via a projector to the participant. The whole cycle requires less than the duration of one imaging volume, allowing for fMRI neurofeedback experiments, during which participants learn to modulate their individual fMRI-derived brain signals, to gain voluntary control over brain processing. (Figure courtesy of Michael Lührs, from http://www.adaptivebrain.eu/Pages/Fellows/MichaelLuehrs.aspx) 
While the advances in treatment of mental health in the last century are humbling, we surely have not reached the end of this journey. Prevalence of mental disorders remain high at an estimated 18-36\% worldwide (Kessler et al., 2011). And, even with a variety of treatment options available, a treatment gap is still existent due to lack of efficacious treatments for some patient groups (Ost, 2008), and underfunding of the mental health sector (Kohn, Saxena, Levav, \& Saraceno, 2004; World Health Organization, 2013). The World Health Organization has therefore called for implementing cost-effective, affordable, and feasible interventions for epilepsy, depression, and psychosis, which are already available (Knapp, Mcdaid, \& Parsonage, 2011; World Health Organization, 2013). It remains to clinical research to further investigate and optimize treatments for other large patient groups, such as patients with somatoform disorders, anxiety disorders, problems with anger management, or addictions, who cannot be optimally treated today. In short, we need other novel treatment options.

\section{Neurofeedback therapy}

Successful clinical applications of EEG-neurofeedback in epilepsy patients, and children with attention-deficit/hyperactivity disorder (Arns, de Ridder, Strehl, Breteler, \& Coenen, 2009; Tan et al., 2009) have spurred interest into the development of other neurofeedback methods, as for example neurofeedback based on functional magnetic resonance imaging (fMRI). Functional magnetic resonance imaging (fMRI) has enabled us to 'look into the brain' while someone is actively engaging in perception, motion, thoughts, or emotion, measuring brain functioning on a millimeter scale across the whole brain. The technique estimates local inflow of oxygen-rich blood by quantifying the amount of oxygenated blood cells through their magnetic properties (Kwong et al., 1992; Ogawa, Lee, Nayak, \& Glynn, 1990). Since its invention in the early 1990s, the number of fMRI studies on human brain processing during cognition, emotion and behavior have increased exponentially (Bandettini, 2012). From the first years of fMRI research, several research groups have been working on the development of real-time fMRI techniques that allow for online data processing and analysis during scanning (Cox, Jesmanowicz, \& Hyde, 1995; Weiskopf, 2012) (figure 4). After the initial introduction of online methods, growing computational power and 
smart algorithms have made real-time fMRI data analysis increasingly powerful (Goebel, 2012; Weiskopf, 2012).

The rapidly increasing knowledge and fast development of real-time fMRI methods has encouraged interest in possible applications, such as facilitating learning in a therapeutic context by providing participants with 'neurofeedback' information on their current brain processes (Goebel, Zilverstand, \& Sorger, 2010; Linden, 2006; Weiskopf, 2012). fMRI technology has given us access to a type of information, which never has been available before, and may be used in a novel way. The general idea is that providing participants with relevant information on ongoing brain processes may enable them to directly and specifically target processes of interest. In a treatment context, the aim is gaining voluntary control over therapeutically relevant processes. Until now, experiments with healthy volunteers have shown that meaningful information can be derived from the fMRI signal, as the modulation of specific brain signals led to related changes in behavior, emotion, and cognition (Goebel et al., 2010; Weiskopf, 2012). In a decade of research, it has been exemplified in healthy participants that improvements in the language, motor and cognitive domain can be achieved by fMRI neurofeedback training, and that the feedback signal was a crucial aspect of learning in these trainings (Goebel et al., 2010; Weiskopf, 2012). It has therefore been shown in first 'proof-of-concept' studies that 'changing the brain' indeed may change cognition and behavior accordingly.

\section{Learning in neurofeedback therapy}

Two different learning mechanisms, reinforcement learning (operant conditioning), and representational learning have been implicated in neurofeedback trainings (Sulzer et al., 2013). While reinforcement learning is the mere associative process of learning stimulus-response mappings, representational learning entails a more elaborate process of extracting useful information from experience, and representing this information in memory, involving more cognitive resources (Sulzer et al., 2013). In the context of a neurofeedback training, reinforcement learning would for instance take place when behavior is strengthened by the presence of a reinforcing stimulus, as for example when the participant achieves the set target level on a visual feedback display. This process would be dominant in a training aiming at up- or down-regulation 
of activation levels within brain regions, or networks implicated in pathology, with a focus on learning to control pathological activation levels during continuous presentation of neurofeedback. Representational learning, on the other hand, is the effortful monitoring and integration of the feedback information over time, putting a higher cognitive demand on the neurofeedback participant, and supporting learning on a higher cognitive, more complex level. This process would be dominant when neurofeedback is used as a tool for shaping a cognitive therapeutic process, as for example during facilitation of learning to apply a cognitive strategy for emotion regulation. Representational learning would also be the dominant learning mechanism when the neurofeedback is presented intermittent, in-between short training periods. Importantly, as the two different learning mechanisms put different cognitive demands on the participants, neurofeedback trainings may be tailored towards the target group by choosing an implementation requiring adequate levels of cognitive demand.

\section{Current research}

In a clinical context, fMRI-based neurofeedback trainings have been first applied in chronic pain patients (DeCharms et al., 2005). Patients had reduced pain symptoms after learning to gain control over activation levels in a brain region involved in conscious pain perception, while being provided continuous neurofeedback (DeCharms et al., 2005). This was the first study to verify that an increase in control on a neural level can directly influence clinical symptoms, and in the following years clinical research on fMRI-based neurofeedback increased rapidly (Sulzer et al., 2013). A second pioneering clinical study demonstrated that tinnitus patients were able to voluntarily attenuate the response of the hyper-activated auditory cortex when provided continuous feedback, which lead to a mild reduction of tinnitus symptoms (Haller, Birbaumer, \& Veit, 2010). Another exemplary trial with Parkinson's patients, established that patients were also able to up-regulate chronically under-activated motor regions following a similar approach, which led to a sustained improvement of symptoms and motor skills (Subramanian et al., 2011). A first clinical study with depressed patients demonstrated that depressed patients improved their ability to generate positive emotions by a fMRI neurofeedback guided training, which led to a reduction of clinical symptoms of depression (Linden et al., 2012). Importantly, this 
study explicitly used neurofeedback as a tool for shaping cognitive strategies, therefore implementing an approach, which exploits both reinforcement and representational learning. The approach was recently replicated in another group of depressed patients (Young et al., 2014). Overall, a number of pioneering clinical applications of $\mathrm{fMRI}$ neurofeedback trainings have thus provided exciting first evidence that neurofeedback may be an efficient tool in a therapeutic process, either as a tool for gaining control over pathological activation levels, or as a tool for shaping cognitive strategies. In the presented research, these studies have been further extended by a neurofeedback training for learning anxiety regulation with intermittent neurofeedback (chapter 4), and a training for learning to up-regulate activation levels in brain regions implicated in attention-deficit/hyperactivity disorder (chapter 5).

Methodological developments for improving online analysis methods of the fMRI signal (Weiskopf, 2012), and the development of new indicators relevant for a therapeutic context are also ongoing (Zilverstand, Sorger, Zimmermann, Kaas, \& Goebel, 2014). The interest in developing new indicators for fMRI neurofeedback trainings stems amongst other from recent clinical neuroscience research showing that brain connectivity biomarkers belong to the most relevant biomarkers for clinical disorders (Bullmore, 2012). While measures of functional brain connectivity have not been used as feedback signals in neurofeedback trainings so far, interest in using them as an extension of currently implemented fMRI online analysis tools has been repeatedly expressed (Goebel et al., 2010; Sitaram et al., 2007; Weiskopf, 2012; Weiskopf et al., 2005), and first implementations have recently been suggested (Koush et al., 2013; Zilverstand et al., 2014). This exemplifies that fMRI neurofeedback trainings are constantly being further developed, with the goal of providing patients with the most relevant information on ongoing brain processes, to maximize information content and optimize learning (Zilverstand et al., 2014). Another important goals are to tailor clinical implementations of $\mathrm{fMRI}$ neurofeedback trainings towards the target group, and ground them theoretically in clinical neuroscience (Linden, 2006). Clinical applications of neurofeedback trainings should be inspired and informed by state-of-the art clinical neuroscience research, as exemplified by an implementation of a fMRI neurofeedback training for anxiety regulation in spider phobia, in which the choice of the feedback target region was based on a study on quantitative representations of subjective anxiety levels in the brain (chapter 3,4 ). In general, aside from methodological improvements of real-time techniques, more clinical 
neuroscience studies elucidating the online mechanisms of therapeutic change, rather than merely examining pre-post treatment differences offline, seem warranted in order to further improve neurofeedback applications (chapter 4).

\section{Research overview}

In the presented PhD thesis different aspects of clinical applications of fMRI neurofeedback are investigated. In chapter 1, a review of fMRI-based neurofeedback approaches in the clinical context at the outset of the presented PhD research is presented. In chapter $\mathbf{2}$, the potential of using functional brain connectivity biomarkers as feedback information in fMRI neurofeedback trainings is investigated. In chapter 3, the representation of subjective anxiety levels in the brain in spider phobia is examined by fMRI, to improve our understanding of the functional roles of the involved brain regions. In chapter 4 , the possibility of enhancing the efficacy of cognitive reappraisal during anxiety provocation by $\mathrm{fMRI}$-based neurofeedback is explored in spider phobia. Finally, in chapter 5 , the effects of self-modulation of anterior cingulate cortex activation levels guided by fMRI neurofeedback is examined in adults with attention-deficit/hyperactivity disorder. All results are discussed with respect to their impact on designing future neurofeedback trainings for therapy. 


\section{References}

Arns, M., de Ridder, S., Strehl, U., Breteler, M., \& Coenen, A. (2009). Efficacy of neurofeedback treatment in ADHD: the effects on inattention, impulsivity and hyperactivity: a meta-analysis. Clinical EEG and Neuroscience, 40(3), 180-189.

Arns, M., Heinrich, H., \& Strehl, U. (2014). Evaluation of neurofeedback in ADHD: the long and winding road. Biological Psychology, 95, 108-15. doi:10.1016/j.biopsycho.2013.11.013

Bandettini, P. a. (2012). Functional MRI: A confluence of fortunate circumstances. Neurolmage, 61, 3-11. doi:10.1016/j.neuroimage.2012.01.130

Beck, A. T. (1967). Depression: Clinical, experimental, and theoretical aspects. New York: Harper \& Row.

Berger, H. (1933). Über das Elektrenkephalogramm des Menschen. Archiv Für Psychiatrie Und Nervenkrankheiten, 87(1), 527-570.

Bewley, T. (2008). Historical background 1780-1840. In Madness to Mental IIIness. A History of the Royal College of Psychiatrists Historical background (pp. 4-9). London: RCPsych Publications.

Bullmore, E. (2012). The future of functional MRI in clinical medicine. Neurolmage, 62(2), 1267-71. doi:10.1016/j.neuroimage.2012.01.026

Butler, A. C., Chapman, J. E., Forman, E. M., \& Beck, A. T. (2006). The empirical status of cognitive-behavioral therapy: a review of meta-analyses. Clinical Psychology Review, 26(1), 17-31. doi:10.1016/j.cpr.2005.07.003

Cox, R. W., Jesmanowicz, A., \& Hyde, J. S. (1995). Real-Time Functional Magnetic Resonance Imaging. Magnetic Resonance in Medicine, 33(2), 230-236. doi:10.1002/mrm.1910330213

DeCharms, R. C., Maeda, F., Glover, G. H., Ludlow, D., Pauly, J. M., Soneji, D., ... Mackey, S. C. (2005). Control over brain activation and pain learned by using realtime functional MRI. Proceedings of the National Academy of Sciences of the United States of America, 102(51), 18626-31. doi:10.1073/pnas.0505210102

English Heritage Foundation. (2012). Disability History. English Heritage Foundation. Retrieved April 01, 2014, from https://www.english-heritage.org.uk

Goebel, R. (2012). BrainVoyager - Past, present, future. Neurolmage, 62(2), 748-756.

Goebel, R., Zilverstand, A., \& Sorger, B. (2010). Real-time fMRI-based brain-computer interfacing for neurofeedback therapy and compensation of lost motor functions. Imaging in Medicine, 2(4), 407-415. doi:10.2217/iim.10.35

Haller, S., Birbaumer, N., \& Veit, R. (2010). Real-time fMRI feedback training may improve chronic tinnitus. European Radiology, 20(3), 696-703. doi:10.1007/s00330-009-1595-z

History of psychiatric institutions. (2014). Wikipedia. Retrieved April 02, 2014, from http://en.wikipedia.org/wiki/

Hoedlmoser, K., Pecherstorfer, T., Gruber, G., Anderer, P., Doppelmayr, M., Klimesch, W., \& Schabus, M. (2008). Instrumental conditioning of human sensorimotor 
rhythm (12-15 Hz) and its impact on sleep as well as declarative learning. Sleep, 31(10), 1401-1408.

Hollon, S. D. (1998). Aaron T. Beck: The cognitive revolution in theory and therapy. In L. G. Castonguay, J. C. Muran, L. Angus, J. A. Hayes, N. Ladany, \& T. Anderson (Eds.), Bringing psychotherapy research to life: Understanding change through the work of leading clinical researchers (pp. 63-74). Washington: American Psychological Association. doi:10.1037/12137-006

Jasper, H., \& Shagass, C. (1941). Conditioning of the occipital alpha rhythm in man. Journal of Experimental Psychology, 28(5), 373-388.

Jones, K. (2000). Insulin coma therapy in schizophrenia. Journal of the Royal Society of Medicine, 93, 147-149.

Jung, R., \& Berger, W. (1979). Fiftieth anniversary of Hans Berger's publication of the electroencephalogram. His first records in 1924-1931. Archiv Für Psychiatrie Und Nervenkrankheiten, 227(4), 279-300. doi:10.1007/BF00344814

Kessler, R. C., Aguilar-Gaxiola, S., Alonso, J., Chatterji, S., Lee, S., Ormel, J., ... Wang, P. S. (2011). The Global Burden of Mental Disorders: An Update from the WHO. Epidemiologia E Psichiatria Sociale, 18(1), 23-33.

Knapp, M., Mcdaid, D., \& Parsonage, M. (2011). Mental Health Promotion and Prevention: The Economic Case. London.

Knott, J. R., \& Henry, C. E. (1941). The conditioning of the blocking of the alpha rhythm of the human electroencephalogram. Journal of Experimental Psychology, 28(2), 134-144.

Kohn, R., Saxena, S., Levav, I., \& Saraceno, B. (2004). The treatment gap in mental health care. Bulletin of the World Health Organization, 82(11), 858-66. doi:/S0042-96862004001100011

Koush, Y., Rosa, M. J., Robineau, F., Heinen, K., W Rieger, S., Weiskopf, N., ... Scharnowski, F. (2013). Connectivity-based neurofeedback: dynamic causal modeling for real-time fMRI. Neurolmage, 81, 422-30. doi:10.1016/j.neuroimage.2013.05.010

Kwong, K. K., Belliveau, J. W., Chesler, D. a, Goldberg, I. E., Weisskoff, R. M., Poncelet, B. P., ... Turner, R. (1992). Dynamic magnetic resonance imaging of human brain activity during primary sensory stimulation. Proceedings of the National Academy of Sciences of the United States of America, 89(12), 5675-9.

Lawton-Smith, S., \& McCulloch, A. (2013). A brief history of specialist mental health services (pp. 1-12). Retrieved from http://www.mentalhealth.org.uk/

Linden, D. E. J. (2006). How psychotherapy changes the brain--the contribution of functional neuroimaging. Molecular Psychiatry, 11(6), 528-38. doi:10.1038/sj.mp.4001816

Linden, D. E. J., Habes, I., Johnston, S. J., Linden, S., Tatineni, R., Subramanian, L., ... Goebel, R. (2012). Real-time self-regulation of emotion networks in patients with depression. PloS One, 7(6), e38115. doi:10.1371/journal.pone.0038115

López-Muñoz, F., \& Alamo, C. (2009). Monoaminergic neurotransmission: the history of the discovery of antidepressants from 1950s until today. Current Pharmaceutical Design, 15(14), 1563-86. 
Mashour, G. A., Walker, E. E., \& Martuza, R. L. (2005). Psychosurgery: past, present, and future. Brain Research Reviews, 48(3), 409-19. doi:10.1016/j.brainresrev.2004.09.002

Mayou, R. (1989). The history of general hospital psychiatry. The British Journal of Psychiatry, 155(6), 764-776. doi:10.1192/bjp.155.6.764

Norman, L. E. (1938). The insulin shock treatment of schizophrenia. The Canadian Medical Association Journal, 39(3), 229-236.

Ogawa, S., Lee, T. M., Nayak, a S., \& Glynn, P. (1990). Oxygenation-sensitive contrast in magnetic resonance image of rodent brain at high magnetic fields. Magnetic Resonance in Medicine, 14(1), 68-78. doi:10.1002/mrm.1910140108

Ost, L.-G. (2008). Cognitive behavior therapy for anxiety disorders: 40 years of progress. Nordic Journal of Psychiatry, 62(1), 5-10. doi:10.1080/08039480802315590

Penney, D., \& Stastny, P. (2006). The Lives They Left Behind. Suitcases from a State Hospital Attic. Retrieved April 01, 2014, from http://www.suitcaseexhibit.org/

Rake's Progress. (2014). Wikipedia. Retrieved April 01, 2014, from http://en.wikipedia.org/wiki/

Rush, A. J., Beck, A. T., Kovacs, M., \& Hollon, S. (1977). Comparative efficacy of cognitive therapy and pharmacotherapy in the treatment of depressed outpatients. Cognitive Therapy and Research.

Shorter, E. (1997a). Alternatives. In A History of Psychiatry: From the Era of the Asylum to the Age of Prozac (pp. 190-238). New York: John Wiley \& Sons.

Shorter, E. (1997b). The Asylum Era. In A History of Psychiatry: From the Era of the Asylum to the Age of Prozac (pp. 33-68). New York: John Wiley \& Sons.

Shorter, E., \& Healy, D. (2007). Shock Therapy. A History of Electroconvulsive Treatment in Mental IIIness. New Brunswick, New Jersey, and London: Rutgers University Press.

Sitaram, R., Caria, A., Veit, R., Gaber, T., Rota, G., Kuebler, A., \& Birbaumer, N. (2007). FMRI brain-computer interface: a tool for neuroscientific research and treatment. Computational Intelligence and Neuroscience, 2007, 25487. doi:10.1155/2007/25487

Sterman, M. B., \& Friar, L. (1972). Suppression of seizures in an epileptic following sensorimotor EEG feedback training. Electroencephalography and Clinical Neurophysiology, 33, 89-95.

Sterman, M. B., Howe, R. C., \& Macdonald, L. R. (1970). Facilitation of spindle-burst sleep by conditioning of electroencephalographic activity while awake. Science, 167(921), 1146-1148.

Subramanian, L., Hindle, J. V, Johnston, S., Roberts, M. V, Husain, M., Goebel, R., \& Linden, D. (2011). Real-time functional magnetic resonance imaging neurofeedback for treatment of Parkinson's disease. The Journal of Neuroscience: The Official Journal of the Society for Neuroscience, 31(45), 16309-17. doi:10.1523/JNEUROSCI.3498-11.2011 
Sulzer, J., Haller, S., Scharnowski, F., Weiskopf, N., Birbaumer, N., Blefari, M. L., ... Sitaram, R. (2013). Real-time fMRI neurofeedback: progress and challenges. Neurolmage, 76, 386-99. doi:10.1016/j.neuroimage.2013.03.033

Tan, G., Thornby, J., Hammond, D. C., Strehl, U., Canady, B., Arnemann, K., \& Kaiser, D. A. (2009). Meta-analysis of EEG biofeedback in treating epilepsy. Clinical EEG and Neuroscience : Official Journal of the EEG and Clinical Neuroscience Society (ENCS), 40(3), 173-179. doi:10.1177/155005940904000310

Thuillier, J. (1999). Ten Years That Changed the Face of Mental IIIness. (D. Healy, Ed.). London: Martin Dunitz Ltd.

UK ECT Review Group. (2003). Efficacy and safety of electroconvulsive therapy in depressive disorders: a systematic review and meta-analysis. Lancet, 361(9360), 799-808. doi:10.1016/S0140-6736(03)12705-5

Weiskopf, N. (2012). Real-time fMRI and its application to neurofeedback. Neurolmage, 62(2), 682-92. doi:10.1016/j.neuroimage.2011.10.009

Weiskopf, N., Scharnowski, F., Veit, R., Goebel, R., Birbaumer, N., \& Mathiak, K. (2005). Self-regulation of local brain activity using real-time functional magnetic resonance imaging (fMRI). Journal of Physiology, Paris, 98(4-6), 357-73. doi:10.1016/j.jphysparis.2005.09.019

World Health Organization. (2013). Investing in mental health: evidence for action. Mental health today (Brighton, England) (pp. 14-23). Geneva, Switzerland. Retrieved from http://www.ncbi.nlm.nih.gov/pubmed/23659013

Young, K. D., Zotev, V., Phillips, R., Misaki, M., Yuan, H., Drevets, W. C., \& Bodurka, J. (2014). Real-time FMRI neurofeedback training of amygdala activity in patients with major depressive disorder. PloS One, 9(2), $e 88785$. doi:10.1371/journal.pone.0088785

Zilverstand, A., Sorger, B., Zimmermann, J., Kaas, A., \& Goebel, R. (2014). Windowed Correlation: A Suitable Tool for Providing Dynamic fMRI-Based Functional Connectivity Neurofeedback on Task Difficulty. PLoS ONE, 9(1), e85929. doi:10.1371/journal.pone.0085929 


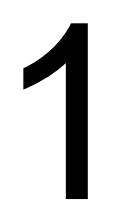

Where are we in fMRI neurofeedback therapy:

"Real-time fMRI-based brain-computer-interfacing for neurofeedback therapy and compensation of lost motor functions"

Based on: Goebel, R., Zilverstand, A., \& Sorger, B. (2010). Real-time fMRI-based brain-computer interfacing for neurofeedback therapy and compensation of lost motor functions. Imaging in Medicine, 2(4), 407-415. 


\section{Summary}

Real-time functional magnetic resonance imaging (rt-fMRI) allows for brain-computer interfaces (BCls) based on hemodynamic brain signals opening up various novel clinical applications. For example, rt-fMRI-based neurofeedback has been suggested as a novel tool for the treatment of neurological and psychopathological disorders. As opposed to conventional offline applications, neurofeedback requires the analysis of fMRI signals online in order to allow providing participants information about their brain activation during an ongoing MRI scan. Recent research supports the idea that an improvement of symptoms of diseases can be achieved if patients are trained with rtfMRI-based neurofeedback to change their brain activation patterns. Rt-fMRI also enables online 'brain reading' applications that can be exploited to develop alternative communication and control devices for patients with severe motor impairments (e.g., 'locked-in' patients). Although other, especially electroencephalography-based $\mathrm{BCl}$ methods have been successfully used in this context, rt-fMRI-based methods may enable robust communication and control in cases where traditional approaches do not provide satisfactory results. 


\section{Introduction}

Since its invention in the early 1990s, functional magnetic resonance imaging (fMRI) has rapidly assumed a leading role among the techniques used to localize brain activation. FMRI is a noninvasive, functional neuroimaging technique that enables the measurement of brain activation in humans and animals in vivo [1]. Generally, neural activation across the whole brain including deep brain structures can be measured simultaneously with relatively high spatial resolution in the range of a few cubic millimeters (the smallest resolved unit is called a volume element or voxel). As the functional measurements are continuously repeated, e.g., every $2 \mathrm{~s}$, a time course of brain activation can be recorded while a participant is actively engaged in a cognitive, emotional, sensory or motor task. So far, fMRI is not known to be associated with any health risks allowing for repeated and frequent scanning of the same participant.

Neuronal activity requires energy and oxygen that is supplied via capillaries in the immediate vicinity of the neurons. When a particular population of neurons becomes active, several subsequent changes in the vascular system take place: At first, a transient local decrease in the blood oxygenation level occurs caused by the oxygen uptake of the neurons that is sometimes observable as an 'initial dip' in the fMRI response. After a short time of about 3 s the increased local neuronal activity also leads to a strong increase in local blood flow. This response of the vascular system to the increased energy demand is called the hemodynamic response. The hemodynamic response not only compensates quickly for the slightly increased oxygen extraction rate but it is so strong that it results in a substantial local oversupply of oxygenated hemoglobin. As oxygenated and deoxygenated hemoglobin have different magnetic properties (susceptibility), the fMRI signal obtained from the particular brain region alters accordingly. This phenomenon was discovered by Ogawa et al. [2] and labeled as the blood oxygenation level-dependent (BOLD) effect. As a result of its functional principles (measuring the neurovascular response rather than neural activity itself), fMRI presents limited spatial and temporal resolution. Moreover, it only provides an indirect measure of neural events. However, several studies that combined intracortical recordings and fMRI have demonstrated a tight coupling between neuronal activity as reflected in local field potentials and the corresponding BOLD signal [3-6].

In conventional/traditional fMRI studies, the obtained data are processed offline. Thus, results become mainly available long after the MRI scanning session has 
actually ended [7]. However, since the mid-1990s, several research groups have been working on the development of real-time fMRI (rt-fMRI) techniques that allow for online data processing and analysis during MRI scanning, immediately after acquisition of a functional image. This became possible through recent technical advances, e.g., in computational power, data acquisition and analysis techniques that have considerably increased the general data processing speed [for reviews of rt-fMRI methods see, e.g., $7,8]$. Introduced by Cox and colleagues [9], rt-fMRI has further substantially improved [10]. Current rt-fMRI procedures include most state-of-the-art data preprocessing and analysis steps of its classical offline counterpart [7]. Figure 1 illustrates the general technical setup and information flow in rt-fMRI experiments.

A variety of potential rt-fMRI applications have been proposed [7, 8], including assurance of data quality and subject compliance during data acquisition, educational purposes, adaptive fMRI experiments and brain-computer interfacing which is the specific topic of the current paper. Generally, a brain-computer interface $(\mathrm{BCl})$ is used to transform brain activation into specific computer signals, such as commands for external devices. Rt-fMRI-based $\mathrm{BCls}$ are currently used in two major ways: One stream of research focuses on the possibility to provide participants with rt-fMRI-based neurofeedback, i.e., information reflecting brain activation in one or more brain regions with the aim to learn to voluntarily modulate it. The second direction deals with the development of alternative communication and control means for patients with severe motor disabilities.

\section{Rt-fMRI-based brain-computer-interfacing for neurofeedback therapy}

A research area emerging in the last years is training and treatment through rt-fMRIbased neurofeedback. Learning based on the highly spatially resolved hemodynamic signal may give way to new neurofeedback applications, complementing older methods as for example electroencephalography-based neurofeedback, which use more global brain signals [11]. In the past ten years, research on rt-fMRI based neurofeedback has moved from feasibility studies that mainly focused on a 'proof of concept' [12-19] towards studies investigating whether normal brain functions can be enhanced in healthy participants [20-23], and towards neurofeedback therapy studies with patients [24, 25] (see Figure 2). These first studies showed that participants were 
able to achieve control over their brain activation [12, 14-18], that rt-fMRI-based neurofeedback was a crucial aspect of learning $[15-18,20,21,24]$, and that participants were able to increase their brain activation linearly throughout the training $[14,15,17,18,20-22,24,25]$. This was remarkable as the total duration of the pure training time of all neurofeedback studies was rather short (six to 30min). In the feasibility studies, the participants were instructed to use simple mental imagery strategies (with the content depending on the target region) as a starting point in the training, and to 'fine-tune' this strategy based on the neurofeedback that they received.

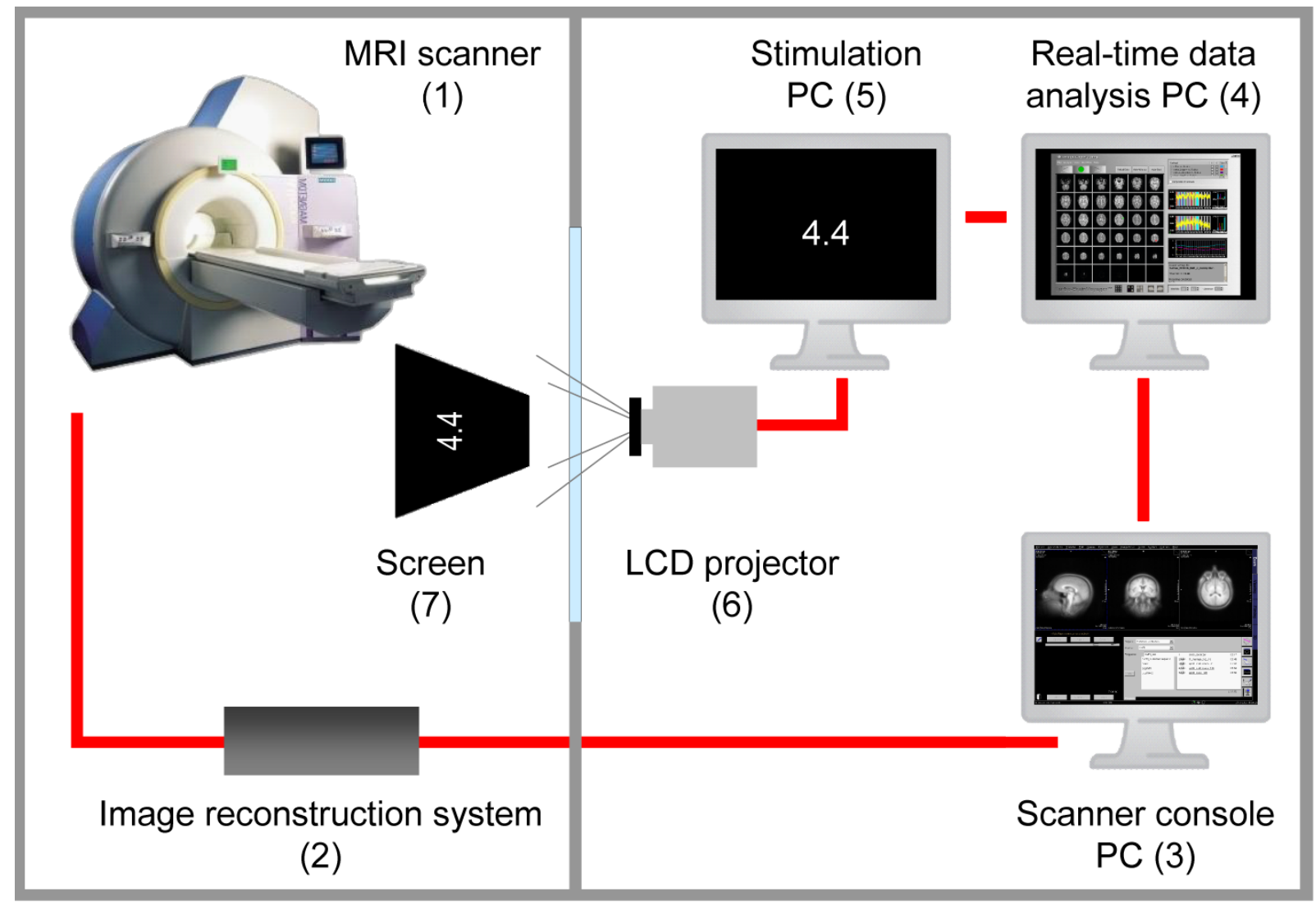

Figure 1. Technical setup and data flow during online/real-time functional MRI.

This figure illustrates the components of the technical setup and the different stages of the data flow during an online/real-time fMRI experiment. Following acquisition of functional data (1), the images are reconstructed (2) and sent to the scanner console's hard disk (3). The PC performing data analysis (4) has instantaneous access to the reconstructed images and the data are being processed. In case of providing neurofeedback information, the analysis software transfers its output to the stimulation PC (5). For example, a custom-developed feedback presentation program generates the visual neurofeedback information (here a numerical representation of the brain activation level) that is presented via the projector (6) on the screen (7) visible by the participant. The whole cycle requires about one TR following data acquisition of a given functional volume. Abbreviations: MRI, magnetic resonance imaging; PC, personal computer; LCD, liquid crystal display; TR, time to repeat. 
In the beginning, the neurofeedback target region was defined based on anatomical criteria [e.g., 14]. In order to optimize the feedback signal, individual target regions were later defined functionally using a task similar to the initial active training strategy.

The success of these feasibility studies lead to a second generation of studies with the goal to modify cognition and behavior through neurofeedback based learning. First studies showed that participants were able to react faster in a finger sequence task and perform better in a word memory task after they learned to up-regulate activation in the supplementary motor area [19, 23]. In a second study it was demonstrated that learning to control the activation of finger and toe representations in motor and somatosensory areas could speed up reaction times in a hand and toe button press task [20]. Additionally it was shown that participants improved their accuracy in a prosodic language task by learning to up-regulate activation in the right inferior frontal gyrus [21]. A recent study investigated whether learning to control amygdala and insula activation through rt-fMRI-based neurofeedback can influence the scores of healthy participants on a mood rating scale [22]. This research may be an important first step towards an rt-fMRI-based neurofeedback treatment for mood disorders. It has long been known that the hyperactivation of the amygdala, as well as the deregulation of brain circuits involved in emotion processing play a crucial role in depression [26]. Amygdala hyperactivation has also been linked to some behavioral patterns observed in depressed patients as for example excessive rumination or the intrusion of emotional memories [26]. Importantly, since pathological brain activation patterns and dysfunctional behavioral patterns are linked, 'changing the brain' in the right direction may change cognition and behavior accordingly. A technique as rt-fMRIbased neurofeedback that gives patients direct access to underlying pathological brain activation could thus be a novel and immediate route in therapy. The most intriguing question is thus, whether it is possible to learn with rt-fMRI-based neurofeedback to substantially influence the complex cognitive processes involved in neurological and psychopathological disorders. If this would be possible, rt-fMRI-based neurofeedback could enable new forms of therapies for patients.

A study that was recently conducted with tinnitus patients showed that rt-fMRIbased neurofeedback learning may directly impact the patients' symptoms. While an rt-fMRI-based neurofeedback study with healthy participants had already shown that it is possible to control activation in auditory brain regions during auditory stimulation [16], the study with tinnitus patients demonstrated that this is also possible when 
tinnitus is the source of a hyperactivation within auditory brain regions [25]. Importantly, a mild decrease of symptoms could be achieved in some patients. Interestingly, in the mentioned studies slightly more complex mental strategies were applied than the ones used in the feasibility studies (that implemented mainly mental imagery). In the study with healthy participants [16], attention manipulation was suggested to control activation in auditory regions during auditory stimulation. In the study with tinnitus patients, participants were advised to use their daily coping strategies [25].

The best known rt-fMRI-based neurofeedback study with patients is the study by deCharms et al. [24]. To investigate the effects of rt-fMRI-based neurofeedback on pain symptoms, this study included healthy participants as well as refractory chronic pain patients, and used several control groups. In healthy participants, the pain was provoked using a hot thermode. All participants were instructed to modulate the activation level in the anterior cingulate cortex through the use of attention-related strategies, reappraisal, and emotion control techniques. This study lead to an interesting observation: While patients primarily learned to increase their brain activation during the training, which was accompanied by an increased pain level, a

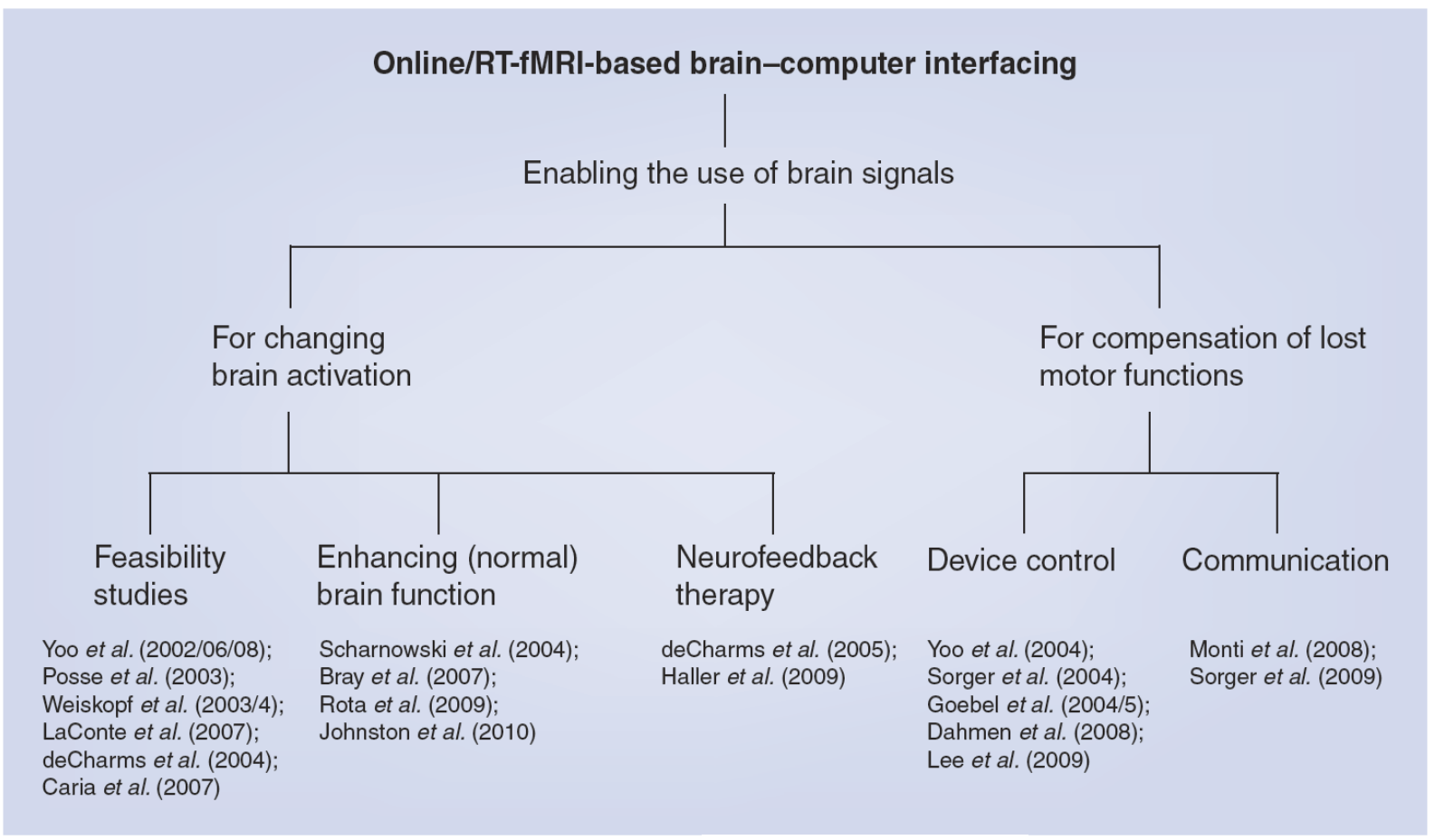

\section{Figure 2. Overview of recent fMRI-based $\mathrm{BCl}$ research.}

The figure provides an overview of the two lines of fMRI-based $\mathrm{BCl}$ research. Moreover, references of corresponding studies are quoted. Abbreviation: fMRI, functional magnetic resonance imaging. 
decrease in pain perception was reported immediately after the training [10]. A 40-60\% reduction of pain symptoms was achieved in the experimental patient group receiving the neurofeedback, while the pain reduction was significantly lower (10-20\%) in the patient control group. Providing patients with the experience that they can cope with an increased level of their symptoms during rt-fMRI-based neurofeedback may be a very potent therapeutic mechanism. Note, however, that the authors themselves noted that there is the possibility that the results of the neurofeedback patient group may be also interpreted as "a trained, controllable form of the typically unconscious placebo effect" [24].

\section{Rt-fMRI-based brain-computer-interfacing for compensation of lost motor functions}

Certain medical conditions (e.g., stroke, progressive neurological diseases) can lead to a central nervous system-induced severe motor paralysis. The extreme case of such motor disability, when a patient suffers from a virtually complete motor deefferentiation, leading to quadriplegia and speech anarthria, has been characterized as the so-called 'locked-in' syndrome [27]. The resulting inability to naturally communicate or to control the environment implies severe ethical and practical problems for affected patients, their relatives and caretakers. Developing motor-independent communication and control means is thus of prime importance. One remaining possibility is the employment of BCls: When the patient's sensory and cognitive functions and associated brain activation are preserved, such that (s)he can intentionally generate distinguishable neuronal responses, a specific coding scheme can be implemented allowing the patient to convey basic thoughts and needs or to control, for example, electromechanical hardware to a limited extent.

One major goal in this context is to increase the number of different commands that can be generated by the $\mathrm{BCl}$ user, measured by the applied brain imaging method, and 'interpreted' (decoded) by the $\mathrm{BCl}$ system as this would increase communication/control flexibility and efficiency. Since human brain functions can be spatially localized and fMRI provides relatively high spatial resolution, this method provides a great opportunity to increase the degrees of freedom in $\mathrm{BCl}$ applications: Separate commands can be encoded by employing different cognitive brain functions. 
Since different cognitive states evoke spatially distinct brain activation patterns that can be disentangled by $\mathrm{fMRI}$ analysis techniques, the original intention of the encoder can be derived.

This possibility has been already tested in several studies with healthy participants. In a pioneering study [28], participants were asked to perform four different mental tasks ('right hand motor imagery', 'left hand motor imagery', 'mental calculation', and 'inner speech') that evoke differential brain activation at four distinct brain locations and were interpreted as four predetermined $\mathrm{BCl}$ commands ("right", "left", "up", and "down"). This allowed the participants to virtually navigate through a simple two-dimensional (2D) maze by solely using repeatedly performed mental processes. Each movement command was based on the average of three trial repetitions and took $2: 15 \mathrm{~min}$. While this study demonstrated feasibility of using rt-fMRI as the basis for a $\mathrm{BCl}$, the transferred information rate was very low (approximately $1 \mathrm{bit} / \mathrm{min})$. Recently, the same research group demonstrated that it is also possible to control 2D movements of a robotic arm by using the same principles [29]. A similar approach was followed by Monti and colleagues [30] in an online fMRI study: Participants were asked autobiographical questions that they were supposed to answer with "yes" or "no" by generating two different mental states ('motor imagery' and 'spatial navigation'). Based on five trial repetitions of the particular mental task, the experimenters were able to infer each participant's answer correctly.

Note however, that only relatively few mental tasks seem to be actually suited to code separate $\mathrm{BCl}$ commands using fMRI signals. So far, only four classes of mental tasks have been successfully explored and employed for this purpose: 'motor imagery', 'spatial navigation', 'mental calculation', and 'inner speech' [28-33]. Thus, additional approaches for increasing the degrees of freedoms for coding separate information units are desired. Therefore, our research group has tested the question whether it might be feasible to hemodynamically encode different commands through using discrete BOLD signal levels. By implementing rt-fMRI-based neurofeedback training, participants succeeded to reach three different target levels within one fMRI session [34]. In a follow-up study, extending the training to four fMRI sessions, up to four levels could be accomplished [35]. However, a reliable differentiation was only possible when data was averaged across many trials, which called the practicability of this approach into question. 
During recent years, our research group has thus concentrated on the possibility to encode a particular $\mathrm{BCl}$ command based on a single mental process only as compared to several trial repetitions which was implemented in all aforementioned studies. That a single internal event can principally be assessed online had been demonstrated before by Posse and colleagues $[13,36]$. In one study, we could demonstrate that participants can play an analogue of the computer game 'pong' simply by adjusting their single-trial brain activation level [37, 38]. In another study [39], participants became able to answer multiple-choice questions (with four answer options) by means of single-trial BOLD responses that were intentionally generated by performing a certain mental task within a particular time window. It is of note that the procedure took less than one minute per answer and did not involve any pretraining as required for most electroencephalography-based communication approaches. Moreover, by further exploiting spatio-temporal BOLD characteristics, we developed a procedure that allows for selecting, hemodynamically encoding and offline decoding any letter of the alphabet based on a single cognitive event [40]. Currently, we are working on setting up an rt-fMRI-based $\mathrm{BCl}$ that allows for reliable letter decoding online, thus enabling participants to communicate any given word during an ongoing MRI session [41].

Brain-computer interfaces based on hemodynamic (vs. neuroelectric) brain signals may constitute an important alternative communication means, for example for patient populations so far not benefiting from electroencephalography-based approaches (e.g., complete locked-in syndrome patients) [see, e.g., 42].

\section{Future perspective}

\section{Rt-fMRI-based neurofeedback therapy}

While it is clear that research on treatment of patients with rt-fMRI-based neurofeedback is still in its early development, the first results convincingly show that this technique may offer fascinating new possibilities. Readjusting the balance in a deregulated brain system as done in the study with tinnitus patients [25] seems to be one possibility. Another idea is that we may change relevant brain systems enduringly simply by using them over and over again in a certain, productive way [43]. This rehearsal of new behaviors may become more effective if patients can be guided with 
rt-fMRI-based neurofeedback in this process. Another promising route to therapy may be that patients can learn to "mimic desired brain states, or mimicking the brain states of others" [10]. This implies of course that we need to be able to define desirable brain states through fundamental research in cognitive and affective neuroscience. Yet another approach to rt-fMRI-based neurofeedback therapy may be to offer patients the possibility to work in a more goal-oriented fashion during therapy. If we would know the neural correlates of therapeutic success in its final stage, we could give patients more freedom in finding their own way towards this goal. This may eventually lead to the discovery of new coping mechanisms. In general it can be concluded that rt-fMRIbased neurofeedback can be used to teach patients how to evoke certain mental states through evoking desirable brain states. However, more cognitive and affective neuroscience research will be necessary to define which brain states correlate to desirable mental states. Since this approach involves a transfer of desirable neural activation states to other participants, further research of advanced brain normalization schemes, such as cortex-based alignment $[44,45]$, is important in order to better relate homologue brain regions across participants' brains. Before regular clinical use, fMRIbased neurofeedback training needs to be evaluated in larger clinical trial studies including appropriate control groups with the goal to assess its effectiveness also with respect to conventional treatment approaches. These trial studies will reveal for which diseases and/or for which patients rt-fMRI-based neurofeedback will be the most appropriate treatment method. Besides the disorders mentioned above (treatment of pain, mood disorders and tinnitus), we expect that several psychiatric conditions will benefit from rt-fMRI-based neurofeedback including particular symptoms of schizophrenia (e.g., reducing the strength of auditory hallucinations), anxiety (e.g., reducing fear during confrontation with anxiety provoking cues), addiction (e.g., reducing craving), and autism (e.g., reducing fear for facial expressions), and reduction of antisocial behavior [46]. For the treatment of some disorders it may turn out that the functional coupling between brain regions is more relevant than information about the mean activation level in small regions or larger networks. Thus, in the future it might be promising to modulate functional connectivity through rt-fMRI-based neurofeedback. 


\section{Rt-fMRI-based communication and control devices}

The success of the first rt-fMRI-based communication tools is based on the rather robust task-related BOLD signal changes, whole-brain coverage and high spatial resolution of fMRI. These properties have been also exploited recently to diagnose the level of awareness in patients with disorders of consciousness [32, 33]. Advances in fMRI data analysis such as better algorithms for the decoding of mental states will further strengthen the role of fMRI for the detection of preserved consciousness in vegetative state patients and for developing robust communication tools for patients with severe motor impairments.

The assignment or 'classification' of activity patterns evoked by mental tasks to specific computer commands or choice selections has been previously performed mostly using signals from pre-localized regions-of-interest (ROIs). An interesting new approach that is currently investigated [47] are multi-voxel pattern classification (MVPC) algorithms that have been used extensively for offline 'brain reading' in recent years [48]. Multi-voxel pattern classifiers are adaptive and learn to associate distributed activation patterns with performed mental tasks. Since multi-voxel pattern classification algorithms are 'learning machines' and more sensitive than univariate (or ROI-based) analyses $[49,50]$, they may lead to $\mathrm{BCls}$ that adapt to the mental states evoked by participants, especially when they are changing over time. In light of these attractive properties, multivariate pattern classifiers are likely becoming an integral component of rt-fMRI-based brain reading systems in the future. Note, however, that good generalization to novel brain states requires that classifiers are trained with a sufficient number of examples before they can assign subsequent brain states to one of the learned mental tasks.

The previous discussions indicates that progress in rt-fMRI-based neurofeedback and communication/external control applications depends not only on technical and methodological advances but also on progress in cognitive and affective neuroscience since improved knowledge of relevant brain structures and their connectivity will be helpful for deciding what brain regions or networks to select in order to optimally achieve certain therapeutic effects - ideally more efficiently than when using traditional (e.g., behavioral) treatments. On the other hand, rt-fMRI-based neurofeedback may also serve as an innovative 'introspective' tool to learn about the functions subserved by specific brain regions. Since participants 'fine-tune' their initially chosen mental tasks to improve voluntary modulation of signals in a targeted brain region, researchers 
can learn about the function of these brain structures by asking participants what they were mentally doing.

\section{From stationary rt-fMRI-based to other mobile hemodynamics-based devices}

While rt-fMRI offers exciting developments for neurofeedback and communication/control devices, $\mathrm{fMRI}$ is an immobile and expensive technology and it would be economically challenging to allow large patient populations to benefit from these innovative tools. There is, however, a related optical technology - functional near infrared spectroscopy (fNIRS) - that measures a similar signal to fMRI [51, 52]. Several studies have already indicated that fNIRS might become a promising additional tool for $\mathrm{BCl}$ purposes [53-55]. It will be important in future years to further investigate the suitability of fNIRS for neurofeedback and communication/external control devices since this technique could be used at the patient's bedside. While fNIRS is portable and much less expensive than fMRI, it only allows, however, measuring activation in brain regions that are rather close to the surface of the head. FNIRS will thus be better suited for replacing fMRI-based communication tools than fMRI-based neurofeedback tools since the latter usually require feedback from rather deep cortical (e.g., cingulate cortex, insula) and sub-cortical brain regions (e.g., amygdala). 


\section{Executive Summary}

- Real-time fMRI (rt-fMRI) differs from other brain-computer interface $(\mathrm{BCl})$ approaches, such as electroencephalography, in providing a high spatial resolution throughout the whole brain allowing for extracting signals from specific cortical and sub-cortical brain regions.

- Learning how to change one's own brain activation pattern through fMRI-based neurofeedback can have a positive impact on cognition and behavior in healthy participants as well as in patients.

- Fundamental research in cognitive and affective neuroscience is necessary to define which brain states are desirable mental states that may be learned through fMRI-based neurofeedback.

- Rt-fMRI-based methods may enable immediate and robust communication and control for 'locked-in' patients, especially in cases in which traditional approaches do not provide satisfactory results.

- Since functional near infrared spectroscopy (fNIRS) is also based on hemodynamic brain signals, it has the potential to bring some benefits of rt-fMRI-based neurofeedback and communication to the patient's bedside. 


\section{Financial disclosure/Acknowledgements}

The first author (RG) is CEO and Chief Software Developer of Brain Innovation, a company that develops neuroimaging software, including "Turbo-BrainVoyager", a software for real-time fMRI data analysis that has been used in several published fMRI neurofeedback studies. Two authors (RG, AZ) gratefully acknowledge the support of the BrainGain Smart Mix Program of the Netherlands Ministry of Economic Affairs and the Netherlands Ministry of Education, Culture and Science. BS was partially funded by the Netherlands Organization for Scientific Research (NWO, Rubicon program, project number: 446-09-010). The authors did not receive writing assistance. 


\section{References}

1. Bandettini, PA, Birn, RM, Donahue, KM: Functional MRI: Background, methodology, limits, and implementation. In: Handbook of Psychophysiology.

Cacioppo, JT, Tassinary, LG, Berntson, GG (Eds.), University Press, Cambridge, 978-1014 (2000).

2. Ogawa, S, Lee, TM, Kay, AR, Tank, DW: Brain magnetic resonance imaging with contrast dependent on blood oxygenation. Proc Natl Acad Sci U S A 87(24), 98689872 (1990).

3. Logothetis, NK, Pauls, J, Augath, M, Trinath, T, Oeltermann, A: Neurophysiological investigation of the basis of the fMRI signal. Nature 412(6843), 150-157 (2001).

4. Logothetis, NK: The neural basis of the blood-oxygen-level-dependent functional magnetic resonance imaging signal. Philos Trans $R$ Soc Lond B Biol Sci 357(1424), 1003-1037 (2002).

5. Logothetis, NK: The underpinnings of the BOLD functional magnetic resonance imaging signal. J Neurosci 23(10), 3963-3971 (2003).

6. Shmuel, A, Augath, M, Oeltermann, A, Logothetis, NK: Negative functional MRI response correlates with decreases in neuronal activity in monkey visual area V1. Nat Neurosci 9(4), 569-577 (2006).

7. Weiskopf, N, Sitaram, R, Josephs, O et al.: Real-time functional magnetic resonance imaging: methods and applications. Magn Reson Imaging 25(6), 9891003 (2007).

8. Bagarinao, E, Nakai, T, Tanaka, Y: Real-time functional MRI: development and emerging applications. Magn Reson Med Sci 5(3), 157-165 (2006).

9. Cox, RW, Jesmanowicz, A, Hyde, JS: Real-time functional magnetic resonance imaging. Magn Reson Med 33(2), 230-236 (1995).

10. deCharms, RC: Applications of real-time fMRI. Nat Rev Neurosci 9(9), 720-729 (2008).

11. Schneider, F, Backes, V, Mathiak, K: Brain imaging: on the way toward a therapeutic discipline. Eur Arch Psychiatry Clin Neurosci 259 Suppl 2, S143-147 (2009).

12. Yoo, SS, Jolesz, FA: Functional MRI for neurofeedback: feasibility study on a hand motor task. Neuroreport 13(11), 1377-1381 (2002).

13. Posse, S, Fitzgerald, D, Gao, $\mathrm{K}$ et al.: Real-time fMRI of temporolimbic regions detects amygdala activation during single-trial self-induced sadness. Neuroimage 18(3), 760-768 (2003).

14. Weiskopf, N, Veit, R, Erb, M et al.: Physiological self-regulation of regional brain activity using real-time functional magnetic resonance imaging (fMRI): methodology and exemplary data. Neuroimage 19(3), 577-586 (2003).

15. deCharms, RC, Christoff, K, Glover, GH, Pauly, JM, Whitfield, S, Gabrieli, JD: Learned regulation of spatially localized brain activation using real-time fMRI. Neuroimage 21(1), 436-443 (2004). 
16. Yoo, SS, O'Leary, HM, Fairneny, T et al.: Increasing cortical activity in auditory areas through neurofeedback functional magnetic resonance imaging. Neuroreport 17(12), 1273-1278 (2006).

17. Caria, $A$, Veit, $R$, Sitaram, $R$ et al.: Regulation of anterior insular cortex activity using real-time fMRI. Neuroimage 35(3), 1238-1246 (2007).

18. Yoo, S, Lee, J, O'Leary, H, Panych, LP, Jolesz, FA: Neurofeedback fMRImediated learning and consolidation of regional brain activation during motor imagery. Int J Imaging Syst Technol 18, 69-78 (2008).

19. Weiskopf, N, Scharnowski, F, Veit, R, Goebel, R, Birbaumer, N, Mathiak, K: Selfregulation of local brain activity using real-time functional magnetic resonance imaging (fMRI). J Physiol Paris 98(4-6), 357-373 (2004).

20. Bray, S, Shimojo, S, O'Doherty, JP: Direct instrumental conditioning of neural activity using functional magnetic resonance imaging-derived reward feedback. $\mathrm{J}$ Neurosci 27(28), 7498-7507 (2007).

21. Rota, G, Sitaram, R, Veit, $R$ et al.: Self-regulation of regional cortical activity using real-time fMRI: the right inferior frontal gyrus and linguistic processing. Hum Brain Mapp 30(5), 1605-1614 (2009).

22. Johnston, SJ, Boehm, SG, Healy, D, Goebel, R, Linden, DE: Neurofeedback: A promising tool for the self-regulation of emotion networks. Neuroimage 49(1), 1066-1072 (2010).

23. Scharnowski, $F$, Weiskopf, $N$, Mathiak, $K$ et al. Self-regulation of the BOLD signal of supplementary motor area (SMA) and parahippocampal place area (PPA): fMRI-neurofeedback and its behavioural consequences. Presented at: 10th Annual Meeting of the Organization for Human Brain Mapping. Budapest, Hungary, 13-17 June 2004.

24. deCharms, RC, Maeda, F, Glover, GH et al.: Control over brain activation and pain learned by using real-time functional MRI. Proc Natl Acad Sci U S A 102(51), 18626-18631 (2005).

25. Haller, S, Birbaumer, N, Veit, R: Real-time fMRI feedback training may improve chronic tinnitus. Eur Radiol 20(3), 696-703 (2009).

26. Davidson, RJ, Pizzagalli, D, Nitschke, JB, Putnam, K: Depression: perspectives from affective neuroscience. Annu Rev Psychol 53, 545-574 (2002).

27. Plum, F, Posner, JB: The diagnosis of stupor and coma. Davis, F.A., Philadelphia, PA, Pages (1966).

28. Yoo, SS, Fairneny, T, Chen, NK et al.: Brain-computer interface using fMRI: spatial navigation by thoughts. Neuroreport 15(10), 1591-1595 (2004).

29. Lee, JH, Ryu, J, Jolesz, FA, Cho, ZH, Yoo, SS: Brain-machine interface via realtime fMRI: Preliminary study on thought-controlled robotic arm. Neurosci Lett 450(1), 1-6 (2009).

30. Monti, MM, Coleman, MR, Owen, AM. 'Brain Reading' with real-time fMRI: communication via detection of brain states in the absence of motor response. 
Presented at: 14th Annual Meeting of the Organization for Human Brain Mapping. Melbourne, Australia, 15-19 June 2008.

31. Boly, M, Coleman, MR, Davis, MH et al.: When thoughts become action: an fMRI paradigm to study volitional brain activity in non-communicative brain injured patients. Neuroimage 36(3), 979-992 (2007).

32. Owen, AM, Coleman, MR, Boly, M, Davis, MH, Laureys, S, Pickard, JD: Detecting awareness in the vegetative state. Science 313(5792), 1402 (2006).

33. Monti, MM, Vanhaudenhuyse, A, Coleman, MR et al: : Willful Modulation of Brain Activity in Disorders of Consciousness. N Engl J Med, (2010).

34. Sorger, B, Bareither, I, Weiskopf, N, Rodriguez, EF, Birbaumer, N, Goebel, R. Voluntary modulation of regional brain activity to different target levels based on real-time fMRI neurofeedback. Presented at: 34th Annual Meeting of the Society for Neuroscience. San Diego, USA, 23-27 October 2004.

35. Dahmen, B, Sorger, B, Sinke, CBA, Goebel, R. When the brain takes BOLD 'steps': Controlling differential brain activation levels via real-time fMRI-based neurofeedback training. Presented at: 14th Annual Meeting of the Organization for Human Brain Mapping. Melbourne, Australia, 15-19 June 2008.

36. Posse, S, Binkofski, F, Schneider, F et al.: A new approach to measure singleevent related brain activity using real-time fMRI: feasibility of sensory, motor, and higher cognitive tasks. Hum Brain Mapp 12(1), 25-41 (2001).

37. Goebel, R, Sorger, B, Kaiser, J, Birbaumer, N, Weiskopf, N. BOLD brain pong: Self regulation of local brain activity during synchronously scanned, interacting subjects. Presented at: 34th Annual Meeting of the Society for Neuroscience. San Diego, USA, 23-27 October 2004.

38. Goebel, R, Sorger, B, Birbaumer, N, Weiskopf, N. Learning to play BOLD Brain Pong: From individual neurofeedback training to brain-brain interactions. Presented at: 11th Annual Meeting of the Organization for Human Brain Mapping. Toronto, Canada, 10-14 June 2005.

39. Sorger, B, Dahmen, B, Reithler, J et al:: Another kind of 'BOLD response': answering multiple-choice questions via online decoded single-trial brain signals. Prog Brain Res 177, 275-292 (2009).

40. Sorger, B, Dahmen, B, Reithler, J, Goebel, R. BOLD communication: When the brain speaks for itself. Presented at: 13th Annual Meeting of the Organization for Human Brain Mapping. Chicago, USA, 2007.

41. Sorger, B, Reithler, J, Dahmen, B, Goebel, R. 'Taking up a dialogue' with the brain: Automated letter decoding from single-trial BOLD responses in real-time. 
Presented at: 15th Annual Meeting of the Organization for Human Brain Mapping. San Francisco, USA, 2009.

42. Birbaumer, N, Cohen, LG: Brain-computer interfaces: communication and restoration of movement in paralysis. J Physiol 579(Pt 3), 621-636 (2007).

43. deCharms, RC: Reading and controlling human brain activation using real-time functional magnetic resonance imaging. Trends Cogn Sci 11(11), 473-481 (2007).

44. Fischl, B, Sereno, MI, Tootell, RB, Dale, AM: High-resolution intersubject averaging and a coordinate system for the cortical surface. Hum Brain Mapp 8(4), 272-284 (1999).

45. Goebel, R, Esposito, F, Formisano, E: Analysis of functional image analysis contest (FIAC) data with brainvoyager QX: From single-subject to cortically aligned group general linear model analysis and self-organizing group independent component analysis. Hum Brain Mapp 27(5), 392-401 (2006).

46. Birbaumer, N. Operant conditioning of the emotional brain in criminal psychopaths. Presented at: 8th Dutch Endo-Neuro-Psycho Meeting. Doorwerth, The Netherlands, 5 June 2009.

47. LaConte, SM, Peltier, SJ, Hu, XP: Real-time fMRI using brain-state classification. Hum Brain Mapp 28(10), 1033-1044 (2007).

48. Haynes, JD, Rees, G: Decoding mental states from brain activity in humans. Nat Rev Neurosci 7(7), 523-534 (2006).

49. Haxby, JV, Gobbini, MI, Furey ML, Ishai A, Schouten JL,, Pietrini, P: Distributed and overlapping representations of faces and objects in ventral temporal cortex. Science 293(5539), 2425-2430 (2001).

50. Kamitani $Y$, Tong F: Decoding the visual and subjective contents of the human brain. nat. Neurosci. 8(5), 679-685 (2005).

51. Irani, F, Platek, SM, Bunce, S, Ruocco, AC, Chute, D: Functional near infrared spectroscopy (fNIRS): an emerging neuroimaging technology with important applications for the study of brain disorders. Clin Neuropsychol 21(1), 9-37 (2007).

52. Villringer, $A$, Chance, B: Non-invasive optical spectroscopy and imaging of human brain function. Trends Neurosci 20(10), 435-442 (1997).

53. Naito, M, Michioka, Y, Ozawa, K, Ito, $\mathrm{Y}$, Kiguchi, M, Kanazawa, T: A Communication Means for Totally Locked-in ALS Patients Based on Changes in 
Cerebral Blood Volume Measured with Near-Infrared Light. IEICE Trans Inf \& Syst E90-D(7), 1028-1037 (2007).

54. Coyle, S, Ward, T, Markham, C, McDarby, G: On the suitability of near-infrared (NIR) systems for next-generation brain-computer interfaces. Physiol Meas 25(4), 815-822 (2004).

55. Sitaram, R, Zhang, H, Guan, C et al.: Temporal classification of multichannel nearinfrared spectroscopy signals of motor imagery for developing a brain-computer interface. Neuroimage 34(4), 1416-1427 (2007).

\section{Reference annotations}

${ }^{*}$ deCharms, RC, Maeda, F, Glover GH et al.: Control over brain activation and pain learned by using real-time functional MRI. Proc Natl Acad Sci U S A. 102, 18626-18631 (2005).

This is the most relevant fMRI neurofeedback studies with patients to date since it a) demonstrated that fMRI neurofeedback is effective in reducing pain perception in patients, and b) compared the findings with several appropriate control groups.

*Owen, AM, Coleman, MR, Boly, M, Davis, MH, Laureys, S, Pickard, JD: Detecting awareness in the vegetative state. Science. 313, 1402 (2006).

This study showed that fMRI can be used to detect consciousness in vegetative state patients exploiting the robustness and whole-brain high-resolution coverage of fMRI.

**Sorger B, Dahmen B, Reithler J et al:: Another kind of 'bold response': Answering multiple-choice questions via online decoded single-trial brain signals. Prog Brain Res 177, 275-292 (2009).

This is the first research article demonstrating a robust possibility to motorindependently communicate basic thoughts and needs based on single-trial fMRI signals.

*Yoo, SS, Fairneny, T, Chen, NK et al.: Brain-computer interface using fMRI: spatial navigation by thoughts. Neuroreport. 15, 1591-1595 (2004).

This is the first fMRI BCl application in which participants performed four different mental tasks to virtually navigate in four directions through a maze; a single action ("up", "down", "left", "right") was performed after analyzing fMRI data of about 2 minutes.

*Weiskopf N, Veit R, Erb M et al.: Physiological self-regulation of regional brain activity using real-time functional magnetic resonance imaging (fMRI): Methodology and exemplary data. Neuroimage 19(3), 577-586 (2003).

This is the first online fMRI neurofeedback study: As opposed to previous applications, feedback was provided for each time point. 


\section{New tools for fMRI neurofeedback:}

"Windowed correlation: a suitable tool for providing dynamic fMRI-based functional connectivity neurofeedback on task difficulty"

Based on: Zilverstand, A., Sorger, B., Zimmermann, J., Kaas, A., \& Goebel, R. (2014). Windowed Correlation: A Suitable Tool for Providing Dynamic fMRI-Based Functional Connectivity Neurofeedback on Task Difficulty. PLOS ONE, 9(1), e85929. 


\section{Abstract}

The goal of neurofeedback training is to provide participants with relevant information on their ongoing brain processes in order to enable them to change these processes in a meaningful way. Under the assumption of an intrinsic brain-behavior link, neurofeedback can be a tool to guide a participant towards a desired behavioral state, such as a healthier state in the case of patients. Current research in clinical neuroscience regarding the most robust indicators of pathological brain processes in psychiatric and neurological disorders indicates that fMRI-based functional connectivity measures may be among the most important biomarkers of disease. The present study therefore investigated the general potential of providing fMRI neurofeedback based on functional correlations, computed from short-window time course data at the level of single task periods. The ability to detect subtle changes in task performance with block-wise functional connectivity measures was evaluated based on imaging data from healthy participants performing a simple motor task, which was systematically varied along two task dimensions representing two different aspects of task difficulty. The results demonstrate that fMRI-based functional connectivity measures may provide a better indicator for an increase in overall (motor) task difficulty than activation level-based measures. Windowed functional correlations thus seem to provide relevant and unique information regarding ongoing brain processes, which is not captured equally well by standard activation level-based neurofeedback measures. Functional connectivity markers, therefore, may indeed provide a valuable tool to enhance and monitor learning within an fMRI neurofeedback setup. 


\section{Introduction}

In neurofeedback training, participants are provided with online feedback on their current individual brain processes. The rationale is that feedback on current brain processing may provide a useful tool for guiding participants towards a desired behavioral state, if the tapped brain correlates are intrinsically linked to relevant phenomena on the behavioral, cognitive and emotional level. Neurofeedback training may thus provide a method for changing brain activation and alleviating symptoms in patients with pathological brain patterns. Preliminary evidence supports the idea that neurofeedback training interventions based on functional magnetic resonance imaging (fMRI) can induce specific changes in behavior, emotion and cognition in healthy participants as well as in patients with psychiatric and neurological disorders $[1,2,3]$. Importantly, previous research demonstrates that effective fMRI-based neurofeedback training is dependent on feeding back the information most relevant for the desired change, for example, giving feedback from brain regions, which are modulated by task performance [2]. However, up to now, different measures derived from fMRI data have not been systematically compared regarding their suitability to provide the most useful or effective feedback. As a first step towards answering this question, the present study aims at investigating whether fMRI-based functional connectivity and activation-level based measures provide the same or different information regarding relevant aspects of different versions of a simple motor task in healthy participants.

The motivation for this study stems from the increased interest in using functional connectivity analysis for investigating biological markers of psychiatric and neurological disorders. It has been claimed that brain connectivity biomarkers are among the most robust indicators of clinical disorders [4]. Deviant functional connectivity patterns in patients have been linked to behavioral, cognitive and emotional symptoms in disorders as diverse as attention deficit hyperactivity disorder [5], schizophrenia [6], autism [7], anxiety [8], mood disorders [9], and movement disorders [10]. For example, research on treatment effects has led to the hypothesis that the most prominent change after successful pharmacological treatment in attention deficit hyperactivity disorder may be the normalization of abnormal taskrelevant functional connectivity patterns, while localized changes of brain activation level seemed to be less indicative [11]. Similarly, a link between a positive treatment response to antidepressant medication and the normalization of cingulate-amygdala 
connectivity has been drawn $[12,13]$. Consequently, the increasing focus on the importance of dysfunctional connectivity networks in psychiatric and neurological diseases during the last decade has also lead to an increased interest in using functional connectivity measures as an fMRI-based neurofeedback signal $[1,2,14,15,16]$. A recent study on the feasibility of using effective connectivity measures within a neurofeedback setup showed that participants are indeed able to voluntarily control such a feedback signal [17]. However, to the best of our knowledge, no systematic comparison regarding the potential of using functional connectivity versus activation level-based measures as a feedback signal in the context of neurofeedback training has been conducted so far.

Functional brain networks emerge when local brain regions interact in order to integrate different task aspects. Two concepts to measure this interaction have been defined in fMRI research: the concept of 'effective connectivity', which aims at measuring how much "influence one neural system exerts over another", and the concept of 'functional connectivity': the mere "statistical dependency among neurophysiological events" [18]. Windowed fMRI correlation measures as implemented here estimate functional connectivity by measuring the amount of common variance in the activation-level changes of two or more circumscribed brain regions during a short time interval. While functional connectivity measures are essentially data-descriptive and not a direct measurement of the underlying neural interactions [18], they have been utilized as an indicator for the ongoing integration on the neuronal level during task performance [19]. Different types of connectivity measures have been categorized into more simple, model-free methods (e.g., correlation) versus more complex modeling methods (e.g., dynamic causal modelling), which may provide more meaningful information (e.g., on the directionality of neural processes), but are computationally more expensive [20]. For real-time data analysis, the feedback signal needs to be computed within relatively short time windows, thus a method providing robust estimates with a few data points may be advantageous in this context. The modeling approach implemented by Koush and colleagues (2013), a dynamic causal modelling approach, required a sliding window with a length of 90 seconds for stable model estimation while statistically stable correlations can be computed based on shorter time windows. Importantly, a systematic comparison regarding the sensitivity of different connectivity methods showed that the correlation method has good sensitivity, performing among the top four of twelve investigated methods [21]. Another 
common division of functional connectivity methods is the separation between hypothesis-driven seed-based methods using a priori-selected regions of interest and data-driven methods that partition the data into functional networks based on statistical criteria, as for example independent component analysis. A systematic comparison between these two sorts of methods using simulations and offline analysis of imaging data showed considerable convergence in the results, both regarding the spatial layout of the functional networks as well as regarding the estimation of connectivity strength [22]. While both types of methods have been made available for real-time data analysis (e.g., sliding window correlation analysis, [23]; sliding window independent component analysis, [24]; single trial-based multi-filter correlation analysis [25]), the hypothesisdriven seed-based methods seem more suitable when the aim is to achieve high spatial specificity within short-time windows. High dimensional independent component analysis, for example, which partitions the data into highly spatially differentiated components, also requires rather long sliding windows. We therefore investigated the potential of using a hypothesis-driven, computationally inexpensive method: windowed seed-based correlation. An important characteristic of this method is that correlation measures have been shown to be susceptible to the influence of noise artifacts $[18,20]$. We therefore employed noise regression, a method which is suitable for real-time data analysis, to remove common noise artifacts $[26,27,28,29,30,31]$.

In order to investigate how well functional connectivity and activation level-based measures may serve as an indicator of subtle changes in task performance, we asked healthy participants to perform a simple finger tapping task that was systematically modulated along two dimensions, namely tapping speed and demand on bimanual coordination, thus combining two aspects of task difficulty (see figure 1). The implementation of the selected task allowed computing individual block-wise performance measures based on both activation levels and functional connectivity, in order to compute brain-behavior correlations. Finger tapping tasks have been well studied regarding their associated effects on functional connectivity, and their effects on the activation level. It has long been known that the activation level within the whole motor network increases with increasing finger tapping speed $[32,33]$, and there is some evidence that increasing demand on bimanual coordination raises activation level as well [34]. Furthermore, the first study on the effects of finger tapping on functional connectivity showed that the correlation between voxels within the motor 
network was higher during task performance than rest [35]. This result has been replicated several times (e.g. $[36,37,38,39]$ ), and extended to show that the functional connectivity between the motor and visual network was higher during a visuo-motor task [40]. Also, a learning experiment on finger tapping [41] has shown that functional connectivity increased during early, as compared to later, learning stages, thus being modulated by general task difficulty. Two other studies provided evidence for a parametric modulation of the correlation strength by finger tapping speed within the motor network [37], and found that differing degrees of demand on bimanual coordination influenced the strength of the functional connection between the bilateral motor regions [36]. Interestingly, they concluded (but did not test) that functional connectivity measures may serve as a better indicator regarding the demand on bimanual coordination than the activation-level based measures. While several studies have thus shown task-dependent modulations of functional connectivity as well as of activation level, none of these studies reported individual brain-behavior correlations that would be needed to evaluate its suitability for real-time analysis. In order to investigate how sensitive and specific these brain activation measures are for detecting the chosen task manipulations in short time windows, we combined, replicated and extended the previous studies by including several different bimanual task variations. All brain measures were derived from short-window time-course data on single-trial level. We hypothesized that while activation-level measures might be a stronger correlate of the individually performed tapping speed, functional connectivity measures might be more sensitive in detecting the demand on bimanual coordination.

\section{Methods}

\section{Participants and Ethics Statement}

Five healthy volunteers participated in the study (mean age: $29.4 \pm 2.8$ years). All participants were right-handed as evaluated by the Edinburgh Handedness Inventory [42]. Participants gave their written informed consent prior to the experiment that was conducted in conformity with the Declaration of Helsinki and approved by the local Ethics Committee of the Faculty of Psychology and Neuroscience at Maastricht University. 


\section{Experimental procedure}

Before the scanning session, participants were trained for half an hour outside the scanner to get familiarized with the motor task. During the MRI session (approx. 2h) a block design with 20-s task blocks and alternating 20-s rest periods was employed. During task blocks participants were guided by a visual metronome that consisted of a flickering ' $R$ ' on the right side (indicating right index finger pace) and a flickering ' $L$ ' on the left side (indicating left index finger pace). This display was generated using the Presentation software package (Version 16, Neurobehavioral Systems Inc., Albany, CA, USA), and was projected onto a mirror mounted in the scanner in front of the participant's head. During the task blocks of a localization run (11 min) participants were instructed to tap at a medium speed of $2 \mathrm{~Hz}$ with their left index finger or their right index

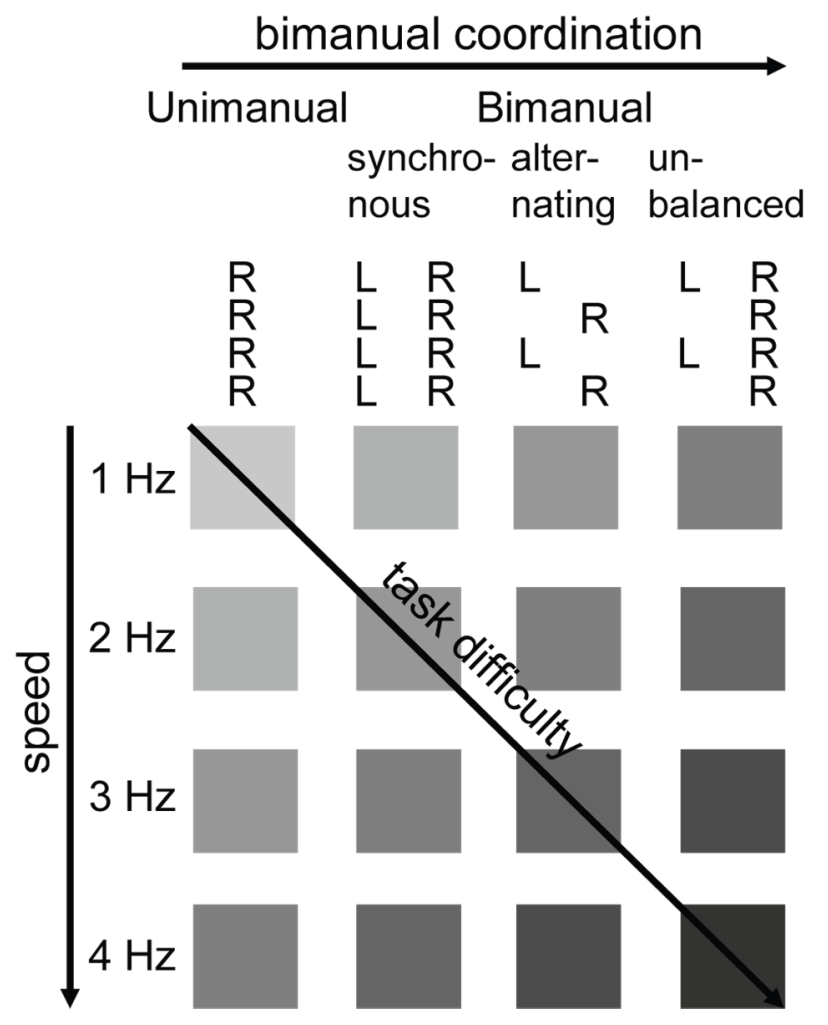

\section{Figure 1. Experimental Design.}

The participants performed four different types of tapping sequences, which were selected to increase demand on bimanual coordination gradually: 1) unimanual: moving only the right index finger, 2) bimanual synchronous: moving both index fingers in synchrony, 3) bimanual alternating: moving both index fingers at the same pace in an alternating fashion, and 4) bimanual unbalanced: moving the left index finger in synchrony with the right index finger, but at half of the pace. Each of these tapping sequences was performed at four different tapping speeds for the right index finger $(1,2,3$, and $4 \mathrm{~Hz})$, which resulted in 16 different experimental conditions. Task difficulty increased along both manipulated task dimensions. 
finger only. During the following eight experimental runs (11 min each) the participants performed four different types of tapping sequences, which required an increasing demand on bimanual coordination: 1) moving only the right index finger (unimanual), 2) moving both index fingers in synchrony (bimanual synchronous), 3) moving both index fingers at the same pace in an alternating fashion (bimanual alternating), and 4) moving the left index finger in synchrony with the right index finger, but at half of the pace (bimanual unbalanced). Each of these tapping sequences was performed at four different right-finger tapping speeds $(1,2,3$, and $4 \mathrm{~Hz})$, which resulted in 16 different experimental conditions (figure 1). Eight repetitions per condition were implemented for each participant (total of 128 task blocks), with the order being counterbalanced across the session. All finger movements during tapping were recorded using a button box, and the software Presentation. The imaging session concluded with the acquisition of the anatomical images.

\section{MRI data acquisition}

The images were acquired at Maastricht Brain Imaging Centre (Maastricht University) on a 3T scanner, (Magnetom Allegra, Siemens Healthcare, Germany), equipped with a standard quadrature birdcage head coil. The participants were placed comfortably in the scanner and their heads were fixed with foam cushions to minimize task-related and other spontaneous motion. All participants were instructed to avoid any movement other than the finger tapping during scanning. Functional images were acquired with a repeated single-shot echo-planar imaging (EPI) sequence with a relatively short repetition time $(T R=1000 \mathrm{~ms})$, adjusted flip angle $\left(F A=62^{\circ}\right)$, standard echo time

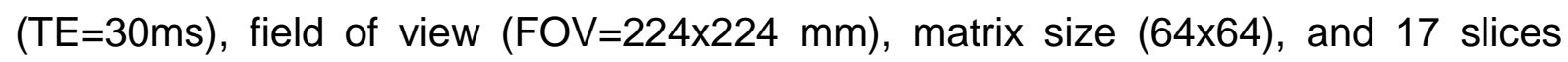
(thickness $=4 \mathrm{~mm}, 1 \mathrm{~mm}$ gap), resulting in a voxel size of $3.5 \times 3.5 \times 5 \mathrm{~mm}^{3}$, ensuring full coverage of the visual, parietal and motor cortices with limited coverage of prefrontal cortex and the cerebellum. Anatomical images were collected with a sequence based on the Alzheimer's Disease Neuroimaging Initiative (ADNI) (parameters: TR=2250ms, $\mathrm{TE}=2.6 \mathrm{~ms}, \quad F A=9^{\circ}, \quad F O V \quad 256 \times 256 \mathrm{~mm}^{2}, 256 \times 256$ matrix, 192 slices, slice thickness $=1 \mathrm{~mm}$, duration=8:26min).

\section{Behavioral data analysis}

The behavioral data were analyzed using custom code in MATLAB (R2010a; The MATHWORKS Inc., Natick, MA, USA) and SPSS Statistics (PASW Statistics 18; IBM 
Corporation, Armonk, NY, USA). As the participants' instruction was to perform the required order of the left and right button presses as accurately as possible, the following types of errors were defined: a) unimanual tapping: a button press with the opposite index finger, b) bimanual synchronous and bimanual unbalanced tapping: a button press that was delayed more than $100 \mathrm{~ms}$ in reference to the button press of the opposite index finger, c) bimanual alternating tapping: each additional consecutive button press. Secondly, the actual individual tapping speed during each of the 128 task blocks was estimated by computing the inter-response intervals between the responses made with the right index finger. Both sets of behavioral measures were analyzed using a general linear model with linear contrasts to test for a linear increase along the dimensions of speed and demand on bimanual coordination. Statistical tests for an increase on demand of bimanual coordination were performed across all tapping sequences, as well as across the three bimanual sequences (testing for a linear increase from bimanual synchronous to alternating to unbalanced). All tests were performed on group-level (fixed effects, the individual block measures were concatenated into one data set), as well as on single-subject level.

\section{MRI data analysis}

Functional and anatomical images were pre-processed and analyzed using BrainVoyager QX (Version 2.3, Brain Innovation B.V., Maastricht, The Netherlands), custom code in MATLAB, and SPSS Statistics. The first five volumes of each functional run were discarded due to $\mathrm{T} 1$ saturation effects. The data was pre-processed using interscan slice-time correction, 3D rigid-body motion correction, and temporal highpass filtering with a general linear model (GLM) Fourier basis set, and up-sampled to $3 \times 3 \times 3 \mathrm{~mm}^{3}$. Noise artifacts were removed from the data using a linear regression approach. Estimated head motion parameters (three translational, three rotational) to model motion artifacts $[28,30]$, a localized estimate of the white matter signal to model scanner artifacts [29], and the ventricular signal to model physiological artifacts, were included in the noise model $[26,27]$. This combination of nuisance regressors has been recommended to be efficient in increasing the specificity $[27,28,30]$, as well as the reliability of the results in functional connectivity analysis [31]. All anatomical and functional data were spatially normalized to Talairach space to enable a comparison between participants [43]. 


\section{Group level functional network analysis}

For the functional network analysis, eight a priori regions of interest (ROIs) were selected based on the reviewed literature and a meta-analysis on finger tapping [44]; left and right primary motor cortex (M1), left and right supplementary motor area (SMA), left and right dorsal premotor cortex (APMC), left and right visual motion area (V5) (figure 2A). All ROIs were defined individually for each participant, based on the data from the localization run, by computing a GLM with the nuisance predictors and task predictors convolved with a standard two-gamma hemodynamic response function. The ROls were defined by selecting the 20 most significant functional voxels from the activation cluster closest to the respective coordinates reported in the metaanalysis [44]. In a second step the average time courses of these ROIs were extracted from the pre-processed and spatially normalized data from the experimental runs. Pearson's correlation coefficients of the activation-level changes in the selected ROIs were computed block-wise for the 128 experimental task blocks of each individual. Three different sets of correlations were calculated using three different time windows: 1) a wider task window that included task on- and offset responses to measure the overall task connectivity (26-s "full task" window, encompassing the rise and decline of the positive BOLD response from 2 seconds after task onset until 28 seconds after, when the decline is expected to level off), 2) a narrow task window omitting task onand offset responses to compute the steady-state task connectivity during continuous task performance (12-s "steady-state" task window, encompassing only the plateau of the BOLD response from 10 seconds until 22 seconds after task onset, to exclude the initial onset and peak), and 3) a narrow rest window to measure rest connectivity (12$\mathrm{S}$ "rest" window, starting 12s before task onset) (figure 2B). Fisher Z transformation was applied to all correlation values to improve the normality of the calculated correlation coefficients [45]. As a preliminary step to the main analysis on singlesubject level, we submitted the block-wise correlations to the same group-level statistical analyses (fixed effects) as the behavioral data.

\section{Within-participant region-of-interest analysis}

Based on the group-level analysis, the functional connection showing the strongest task modulation was selected in order to further investigate the feasibility of using functional connectivity measures as a neurofeedback measure on a single-subject level. Visual inspection of the single-block blood oxygenation level-dependent (BOLD) 
A. Functional Network

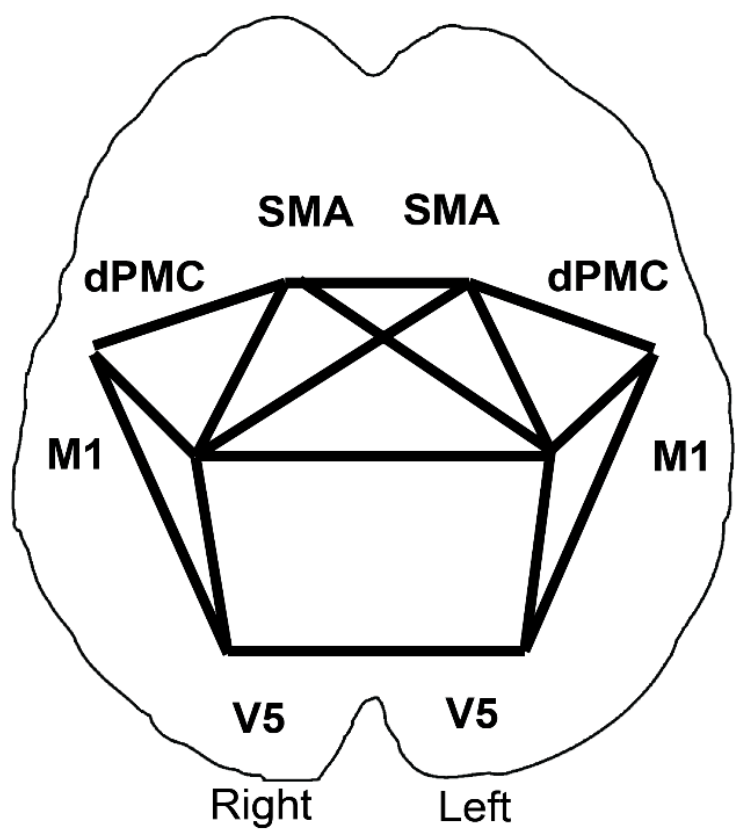

B. Time Windows

26-s task window

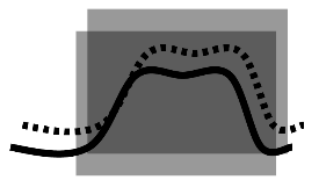

12-s task window

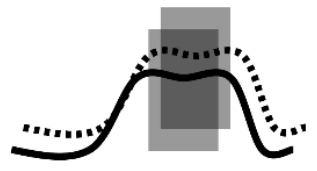

12-s rest window

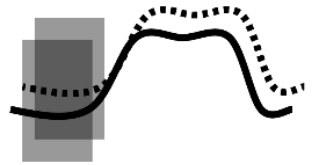

Figure 2. Schematic representation of functional network.

All fMRI-based measures were derived from a network of a priori selected regions of interest (M1 = primary motor cortex, $\mathrm{dPMC}=$ dorsal premotor cortex, $\mathrm{SMA}=$ supplementary motor area, V5 = visual motion area), which are depicted schematically in panel $\mathbf{A}$. The time windows (grey boxes) used in the functional connectivity analysis are superimposed on the schematic BOLD responses of the two regions of interest (solid and dotted line) in panel B.

responses from the selected ROIs confirmed that the steady-state task- and restconnectivity (12-s steady-state task window and 12-s rest window) were not contaminated by the task on- and offset responses. For the block-wise correlations the same statistical analyses as performed at the group-level were repeated at the singlesubject level. Additionally, block-wise activation level measures were computed for all 128 task blocks (\% signal change 12-s task vs. 12-s rest), and submitted to the same statistical analysis. Furthermore, we investigated two different types of brain-behavior link. First, to evaluate the sensitivity and specificity of the computed brain measures to detect if a task was performed uni- or bimanually, we set the intermediate value between the means from both types of tasks as a threshold. We then computed how well the actually performed task could be detected based on the single-block brain measures using this simple threshold approach (chance level being $50 \%$ ). We tested for significance through paired t-tests. Second, to estimate the criterion validity of the brain measures for indicating which tapping speed was performed, we correlated the 
block-wise brain measures with the block-wise actual finger tapping speed. We tested for the significance of this correlation through linear regression. Finally, to directly test for differences between the different sets of functional connectivity and activation levelbased measures statistically, we submitted them pairwise to a three-way (measure $x$ tapping speed $x$ demand on bimanual coordination) analysis of variance (ANOVA) for repeated measures on group (fixed effects) and single-subject level.

\section{Results}

\section{Behavioral data}

All participants completed the session as intended. The linear modulation of actual tapping speed of the right index finger was highly significant in all participants (Group: $F(1,39)=748, p<0.001$, Single Subject: $F(1,7)>2850, p<0.0001)$, indicating that there was a linear increase in performed tapping speed as required (figure $3 \mathrm{~A}$ ). Also, as expected, a significant linear increase in error rate was found in all participants when demand on bimanual coordination increased across the four different types of tapping sequences (Group: $F(1,39)=1997, p<0.001$, Single Subject: $F(1,7)>545, p<$ 0.0001 ), or across the three bimanual tapping sequences (Group: $F(1,39)=1335, p<$ 0.001 , Single Subject: $F(1,7)>239, p<0.0001$ ) (figure $3 B$ ). The modulation of error rate by tapping speed was not linear. In four participants, the results showed a significant quadratic effect of speed, with most errors being made during the slowest and the fastest conditions (Group: $F(1,39)=7.9$, Single Subject: $F(1,7)>9.2, p<$ 0.019). Finally, the behavioral data from these four participants showed a significant interaction effect between demand on bimanual coordination and actual tapping speed (Group: $F(1,39)=6.0$, Single Subject: $F(1,7)>29.2, p<0.001$ ), with a stronger modulation of error rate by demand on bimanual coordination when the tapping speed was higher. 
A

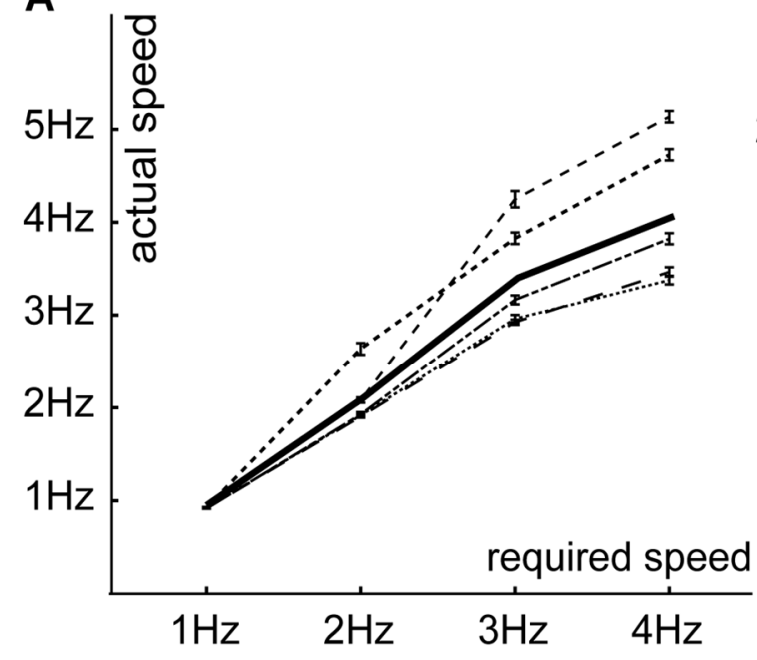

B

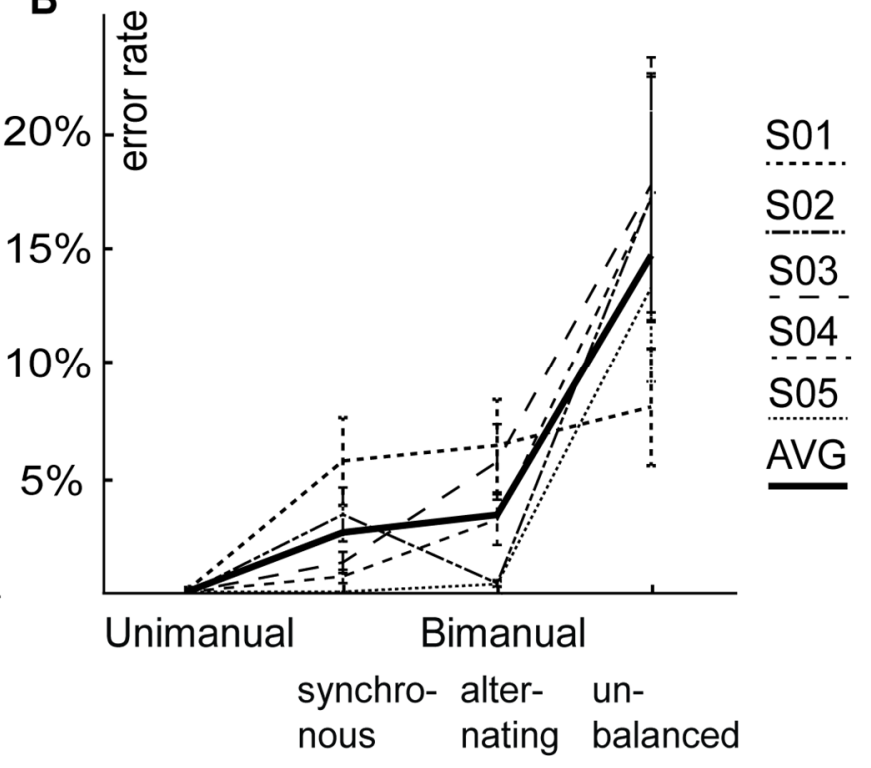

\section{Figure 3. Behavioral results.}

The behavioral results showed a significant linear increase of tapping speed consistent with the experimental manipulation for all participants. The actual tapping speed of the right index finger from all individuals (S01-05, mean \pm individual SE), as well as the average (AVG) is plotted dependent on the required speed in panel $\mathbf{A}$. Second, there was a significant linear increase of error rate with increasing demand on bimanual coordination (from left to right) in all participants. In panel $\mathbf{B}$ the individual error rate (S01-05, mean \pm individual SE), and average (AVG) is plotted for the four performed tapping sequences.

Table 1. Individual regions-of-interest.

\begin{tabular}{|c|c|c|c|c|c|c|c|c|c|c|c|c|c|c|c|c|c|c|c|c|c|c|c|c|}
\hline & \multicolumn{3}{|c|}{ left M1 } & \multicolumn{3}{|c|}{ right M1 } & \multicolumn{3}{|c|}{ left SMA } & \multicolumn{3}{|c|}{ right SMA } & \multicolumn{3}{|c|}{ left dPMC } & \multicolumn{3}{|c|}{ right dPMC } & \multicolumn{3}{|c|}{ left V5 } & \multicolumn{3}{|c|}{ right V5 } \\
\hline & $x$ & $\mathbf{y}$ & z & $\mathbf{x}$ & y & $z$ & $\mathbf{x}$ & y & z & $\mathrm{x}$ & y & $\mathbf{z}$ & $\mathbf{x}$ & $y$ & z & $\mathbf{x}$ & $y$ & $z$ & $x$ & y & $z$ & $\mathrm{x}$ & y & z \\
\hline Participant 01 & -39 & -24 & 48 & 40 & -22 & 46 & -8 & -12 & 52 & 3 & -10 & 57 & -49 & -13 & 44 & 50 & -9 & 47 & -46 & -63 & 3 & 40 & -62 & 4 \\
\hline Participant 02 & -35 & -18 & 54 & 33 & -22 & 50 & -6 & -8 & 56 & 1 & -2 & 60 & -48 & -10 & 49 & 42 & -5 & 45 & -55 & -61 & 2 & 37 & -66 & -1 \\
\hline Participant 03 & -37 & -17 & 51 & 35 & -17 & 56 & -7 & 1 & 47 & 5 & 10 & 43 & -36 & -1 & 55 & 35 & -3 & 52 & -42 & -73 & 2 & 49 & -67 & 0 \\
\hline Participant 04 & -37 & -15 & 58 & 37 & -12 & 50 & -5 & -6 & 51 & 3 & -3 & 51 & -44 & -4 & 51 & 47 & 1 & 52 & -44 & -75 & -6 & 45 & -73 & -4 \\
\hline Participant 05 & -41 & -13 & 51 & 35 & -18 & 50 & -6 & 0 & 54 & 7 & 0 & 53 & -47 & -6 & 53 & 43 & -5 & 48 & -45 & -63 & 5 & 43 & -69 & 5 \\
\hline Group & -38 & -17 & 52 & 36 & -18 & 50 & -6 & -5 & 52 & 4 & -1 & 53 & -45 & -7 & 50 & 43 & -4 & 49 & -46 & -67 & 1 & 43 & -67 & 1 \\
\hline Meta-analysis & -38 & -26 & 50 & 36 & -22 & 54 & -4 & -8 & 52 & & & & 47 & 1 & 50 & & & & -42 & -66 & 4 & 44 & -66 & -2 \\
\hline
\end{tabular}

The Talairach coordinates of the functionally defined regions of interest are listed for each participant. The Talairach coordinates for the group average and coordinates reported by a meta-analysis of 38 finger tapping studies [44] are shown for comparison. M1 = primary motor cortex, $\mathrm{SMA}=$ supplementary motor area, $\mathrm{dPMC}=$ dorsal premotor cortex, $\mathrm{V} 5=$ visual motion area. 


\section{Group-level functional network}

In all participants the eight ROIs were functionally localized based on the independent data from the localization run (table 1). In the functional network derived from the rest periods immediately preceding each task block (12-s rest window) only one of the fifteen analyzed connections was significantly linearly modulated by tapping speed in the preceding task period (left-right SMA: $F(1,39)=8.2, p=0.007$, not depicted), and demand on bimanual coordination (left-right SMA: $F(1,39)=9.5, p=0.004$, not depicted). In the analysis of the functional connectivity networks derived from the two task windows (26-s full task and 12-s steady-state task window), a number of connections showed a significant increase of functional connectivity with increasing tapping speed (e.g., left-right M1 26-s full task window: $F(1,39)=26.6, p<0.001,12-$ s steady-state task window: $F(1,39)=6.7, p=0.01$ ), and increasing demand on bimanual coordination (e.g., left-right M1 unimanual vs. bimanual 26-s full task window: $F(1,39)=523.5, p<0.0001,12-s$ steady-state task window: $F(1,39)=18.5, p<0.001$; left-right M1 linear modulation 26-s full task window: $F(1,39)=462.0, p<0.0001,12-s$ steady-state task window: $F(1,39)=28.6, p<0.001$ ) (figure $4 A$, 3B). In general, the

\section{Figure 4. Group-level functional network results.}

The task-dependent modulation of the group-level overall task connectivity (26-s full task window, depicted on the left) and the steady-state task connectivity (12-s steady-state task window, shown on the right) are visualized schematically for the investigated functional network $(\mathrm{M} 1=$ primary motor cortex, $\mathrm{dPMC}=$ dorsal premotor cortex, SMA = supplementary motor area, V5 = visual motion area). The upper row shows the significant difference in functional connectivity between unimanual and bimanual tapping. The second row depicts the significant linear increase of functional connection with increasing demand on bimanual coordination during bimanual tapping. The third row shows how connectivity significantly increased with increasing tapping speed. The bottom row depicts the significant interaction effects between demand on bimanual coordination and tapping speed. While the effects were weaker during steady-state in comparison to overall task connectivity, the task-dependent modulations were qualitatively very similar independent of the time window used. Only one connection, the connection between the two primary motor cortices, showed all effects independent of the time window used. This connection also showed the highest average correlation in the functional network (the thickness of the depicted connections equals the average correlation across all experimental conditions). 
A 26-s task window
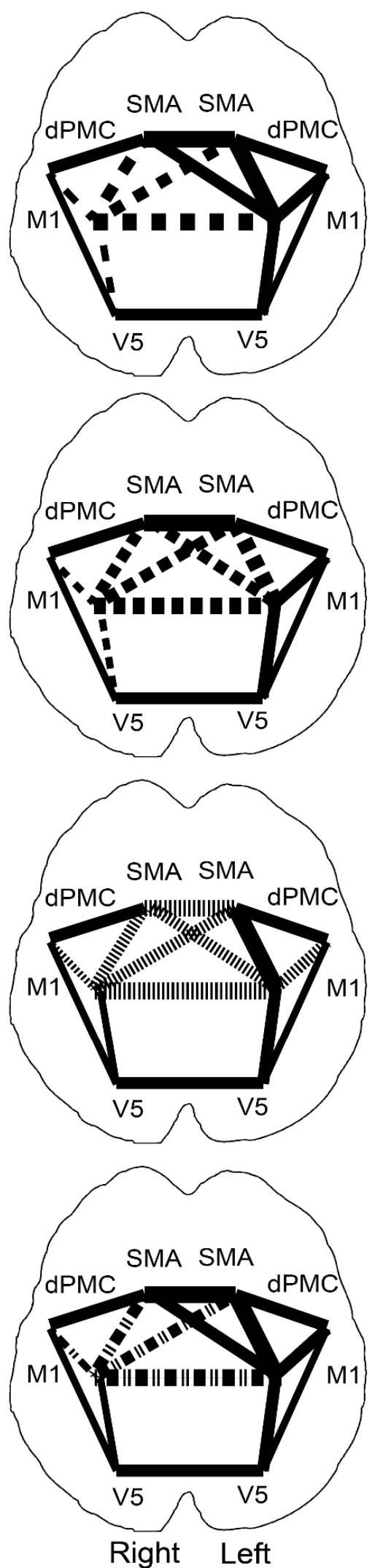

B 12-s task window
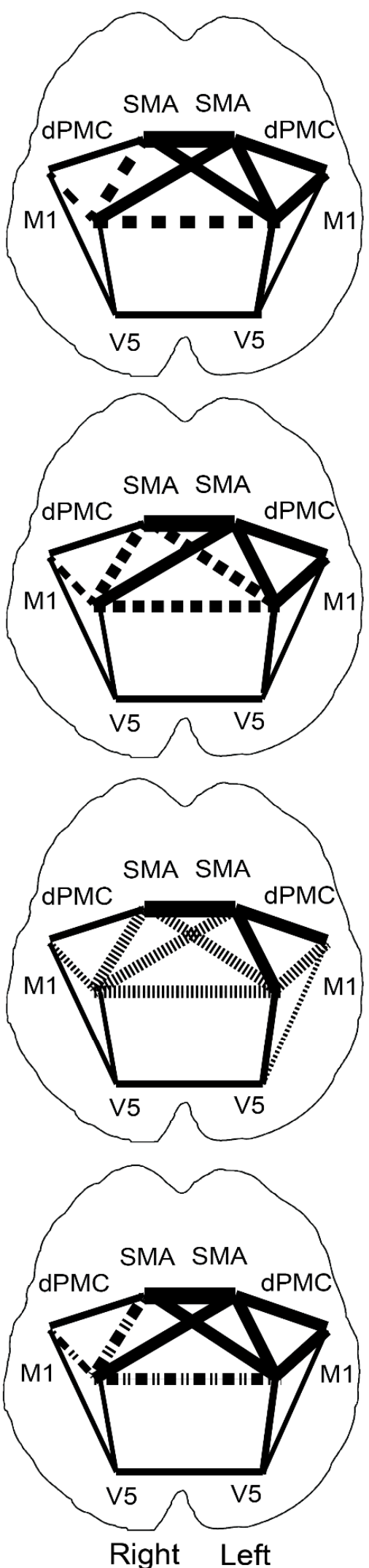

bimanual coordination

bimanual $>$ unimanual

$\because-\bar{r} r>0.0$

$\because-r>0.2$

- $r>0.4$

I I I $r>0.6$

no significant difference

$r>0.0$
$r>0.2$
$r>0.4$
$r>0.6$

bimanual coordination

linear increase bimanual

.... $r>0.0$

$\operatorname{mar} r>0.2$

IIII $r>0.4$

IIII $r>0.6$

no significant increase

$r>0.0$
$r>0.2$
$r>0.4$
$r>0.6$

tapping speed

linear increase

........... $r>0.0$

เแแแ

||I|||||||||| $r>0.4$

||I||||||||||| $r>0.6$

no significant increase

$\begin{aligned} r & >0.0 \\ r & >0.2 \\ r & >0.4 \\ r & >0.6\end{aligned}$

bimanual coordination \& tapping speed significant effects

" - - , $r>0.0$

"I-" $r>0.2$

II $r>0.4$

||⿴囗|⿲| $\mid r>0.6$

not significant

$r>0.0$

$r>0.2$

$r>0.4$ 
task effects in the steady-state task functional network (12s task window) were weaker but not qualitatively different from the effects found in the overall task connectivity functional network (26s task window) (figure 4A, 3B). Finally, three connections of the functional networks derived from the task data showed significant interaction effects for both time windows (left M1 with right M1, left M1 with right SMA, and left V5 with left dPMC). For these three connections the functional connectivity was highest when both tapping speed and demand on 9.0, $p=0.005$, 12-s steady-state task window: $F(1,39)=5.3, p=0.027)$. A comparison between all functional connections showed that the task-dependent effects were strongest for the left-right M1 connection. Further analyses were therefore restricted to this functional connection.

\section{Figure 5. Within-participant region-of-interest results.}

The individually selected regions of interest in the left and right primary motor cortices (M1) of the five participants are projected onto an average of all participants' anatomical brain images in panel $\mathbf{A}$ ( $z=51$, Talairach space), and onto the individual anatomical brain images in panel B. In Panel C the BOLD responses from left M1 (averaged across all tasks) are depicted for all participants (mean \pm individual SE). The time windows used to compute the block-wise correlations are superimposed on the BOLD responses. Panel D displays the average activation level (group mean \pm group SE) during each of the sixteen experimental conditions (four different tapping sequences performed at four different speeds) in right and left M1 (group mean \pm group SE), while panel $E$ shows the results (group mean \pm group SE) from the correlation analysis of the same regions of interest. From unimanual to bimanual finger tapping the average activation level increased, as expected, in the right, but not left primary motor cortex (left M1: unimanual $1.4 \%$, synchronous $1.3 \%$, alternating $1.2 \%$, unbalanced $1.2 \%$; right M1: unimanual $-0.2 \%$, synchronous $1.3 \%$, alternating $1.2 \%$, unbalanced $0.9 \%$ ). This effect was reflected in the steady-state task and overall task connectivity (26-s full task window: unimanual: 0.02 , synchronous 0.75 , alternating 0.73 , unbalanced $0.73 ; 12$-s steady-state task window: unimanual: 0.24 , synchronous 0.42 alternating 0.47 , unbalanced 0.47 ,), but not visible during rest connectivity (unimanual 0.40 , synchronous 0.49 alternating 0.48 , unbalanced 0.48 ). Additionally, all task derived measures were modulated by finger tapping speed. For the activation level derived measures, this effect was most pronounced when the performed tapping sequence was easy. During steady-state connectivity the modulation by finger tapping was strongest during unimanual, alternating and unbalanced tapping, and for the overall task connectivity the modulation by finger tapping speed was most pronounced as tapping sequences became most difficult (alternating and unbalanced tapping). 
A

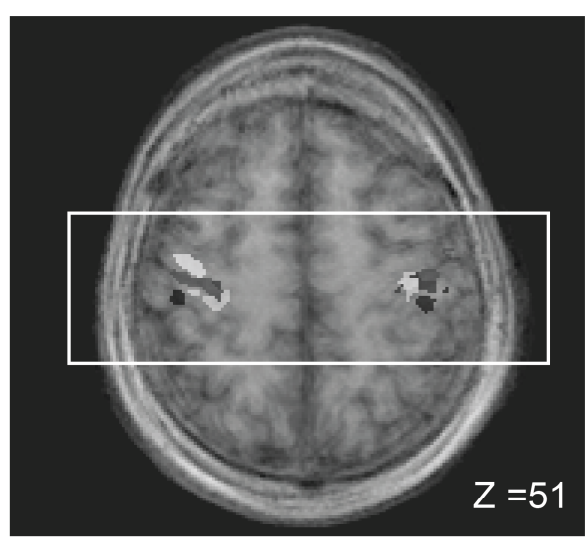

B

Participant 01

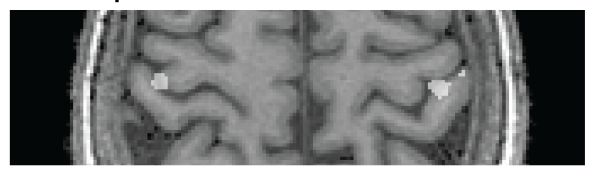

Participant 02

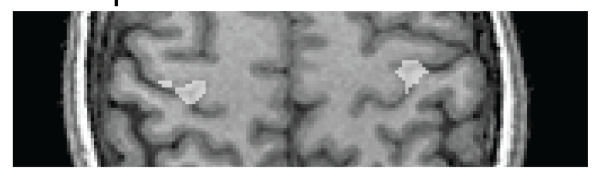

Participant 03

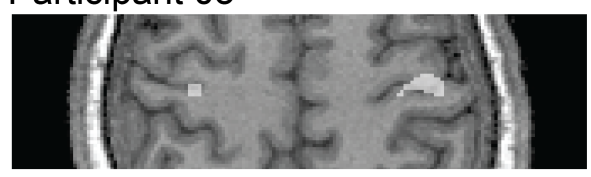

Participant 04

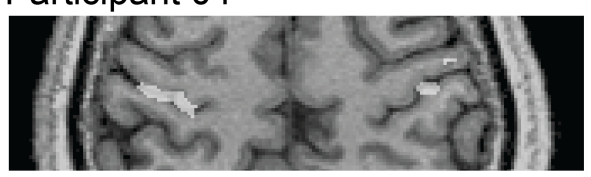

Participant 05

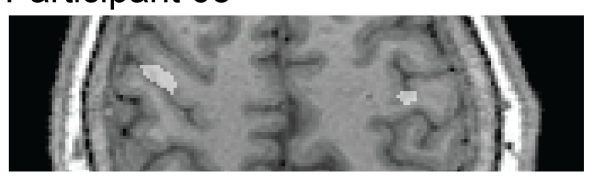

$Z=51$
C

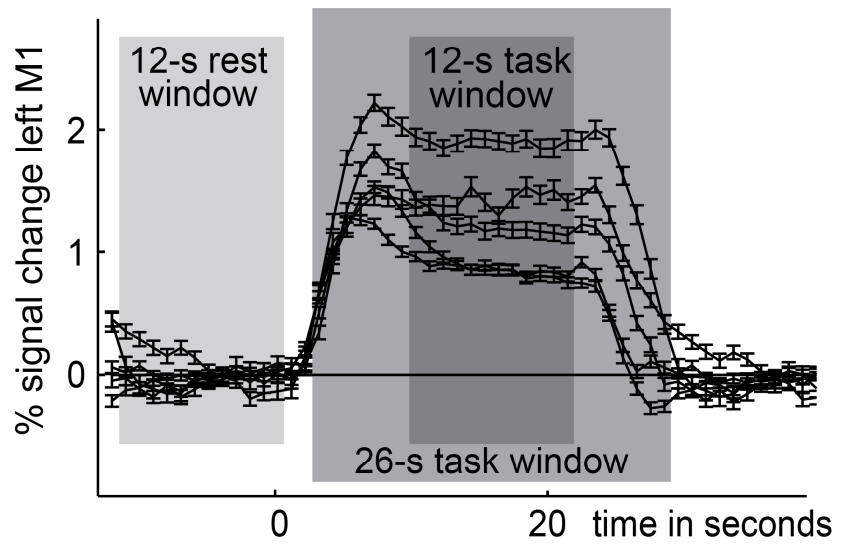

D $\%$ signal change

left M1

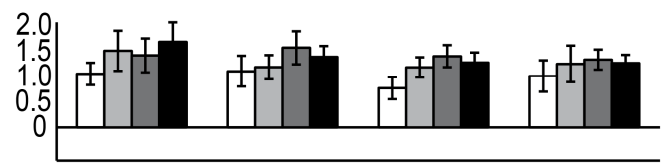

right $\mathrm{M} 1$

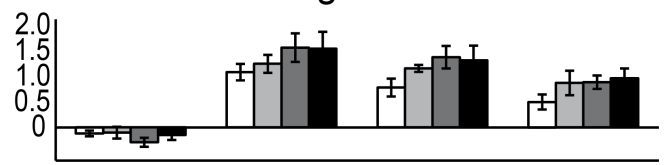

E

Correlation left-right M1

26-s task window
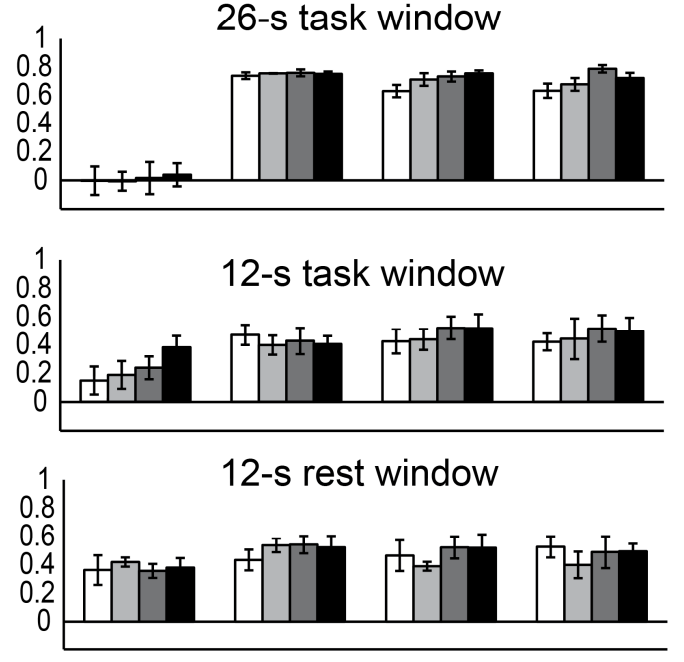

Unimanual Bimanual

synchro- alter- un-

nous nating balanced

\section{$\square$}

$1 \mathrm{~Hz} 2 \mathrm{~Hz} 3 \mathrm{~Hz} 4 \mathrm{~Hz}$ 


\section{Within-participant region-of-interest}

Figure 5 depicts the individually localized left and right primary motor cortex, the measured BOLD responses from these regions with the functional connectivity analysis time windows superimposed, as well as the group-average activation-level and correlation values derived from this data. The statistical analysis of the individual block-wise activation level measures showed that there was a slight decrease in activation level in the left primary motor cortex with increasing demand on bimanual coordination in four out of five participants (Group: $F(1,39)=14.6, p<0.001$, Single Subject: $F(1,7)>6.1, p<0.04)$. Furthermore, as expected, the activation level of this region linearly increased when tapping speed increased, reaching significance in four participants (Group: $F(1,39)=37.2, p<0.001$, Single Subject: $F(1,7)>7.6, p<0.03$; figure 5D). There was no significant interaction effect except in one participant (Group: $F(1,39)=1.9, p=0.17$, Single Subject: $F(1,7)=7.0, p=0.03)$. In the right primary motor cortex, as expected, there was a strong increase in activation level between unimanual and bimanual tapping (Group: $F(1,39)=297, p<0.001$, Single Subject: $F(1,7)>86, p$ $<0.001$, figure 5D, 6A), but activation levels decreased with increasing demand on bimanual coordination across the three bimanual tasks in four participants (Group: $F(1,39)=297, p<0.001$, Single Subject: $F(1,7)=p<0.001$, figure 5D). In both regions of interest the highest activation levels were thus found when finger tapping speed was high and an easier tapping sequence was performed. The interaction effect in the right primary motor cortex was significant in one participant (Group: $F(1,39)=8.7, p=0.005$, Single: Subject: $F(1,7)=23.0, p=0.002)$. The individual analyses of the functional connectivity measures on single-subject level confirmed the reported group results, showing robust and consistent differences in task connectivity between the unimanual vs. the bimanual tapping sequences (26-s full task window: all participants, $F(1,7)>$ 54.4, $\mathrm{p}<0.001$; 12-s steady-state task window: three participants, $F(1,7)>5.9, p<$ 0.04, 12-s rest window: one participant, $F(1,7)=20.6, p=0.003$, table 2, figure $5 E$, 6). The average sensitivity and specificity in detecting if a task was performed uni- or bimanually was high when based on the activation-level measures from the right primary motor cortex ( $86 \%$ sensitivity, $91 \%$ specificity), and the overall task connectivity measures $(93 \%, 91 \%)$, and moderate when based on the steady-state task connectivity measures (68\%, 59\%) (table 2, figure 6). Second, as in the grouplevel analysis, task connectivity also increased when tapping speed increased. This 


\section{A \% signal change}

\section{Participant 03}

$83 \%$ sensitive, $100 \%$ specific

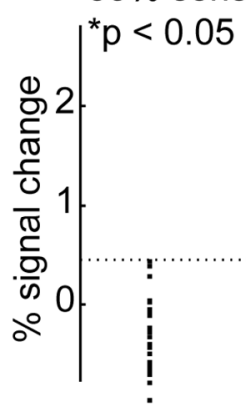

Participant 05

$90 \%$ sensitive, $84 \%$ specific

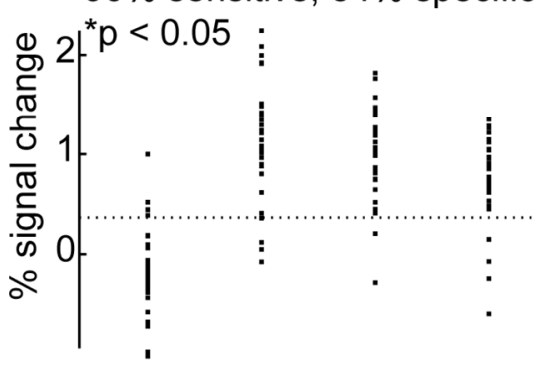

Unimanual Bimanual

synchro- alter- un-

nous nating balanced
B

Correlation 26-s task window

$96 \%$ sensitive, $97 \%$ specific ${ }^{*} p<0.05$

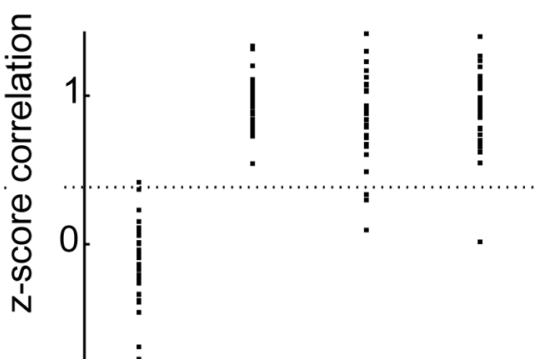

$92 \%$ sensitive, $97 \%$ specific

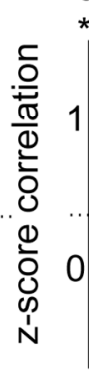

$p<0.05$

\section{Correlation 12-s task window}

$63 \%$ sensitive, $63 \%$ specific ${ }^{*} p<0.05$

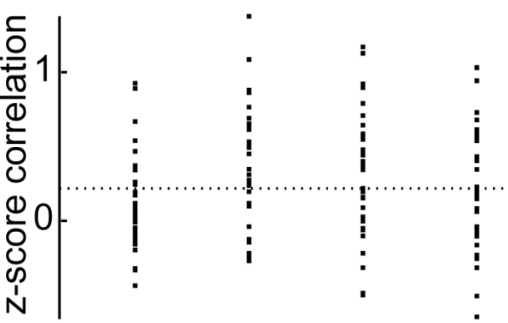

$64 \%$ sensitive, $59 \%$ specific $p>0.05$

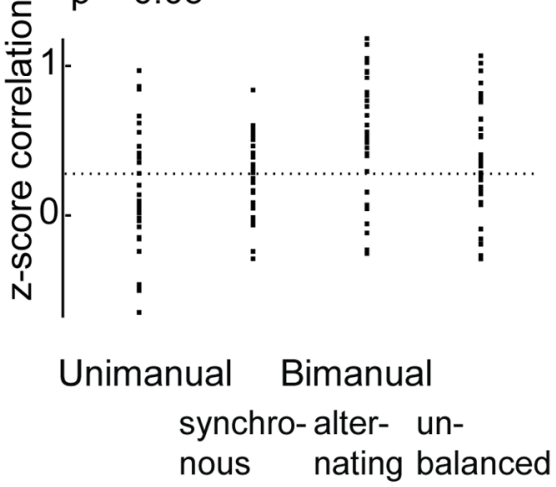

\section{Figure 6. Sensitivity and specificity in detecting bimanual tapping.}

The sensitivity and specificity in detecting if a task was uni- or bimanually performed was computed using a simple threshold approach. The results for the block-wise activation-level measures from right M1 (panel A), and the 26-s full task correlations (panel B), and the 12-s steady-state task correlations (panel C) are presented for two participants. Each dot represents one block. Significant results are marked with an asterisk. Activation level based and overall task connectivity measures both performed well in making this binary decision, while steady-state connectivity measures performed more poorly, but still above chance level (50\%) in three of five participants (see table 2 ).

linear effect was significant for both the 26-s full task window (three participants, $F(1,7)$ $>6.1, \mathrm{p}<0.04$ ) and the 12-s steady-state task window (two participants, $\mathrm{F}(1,7)>4.9$, $p<0.002)$, and non-significant during rest $(F(1,7)<1.8, p>0.22)$. However, the individual functional connectivity analyses showed an interaction effects for four participants with the 26-s full task window $(F(1,7)>6.5, p<0.04)$, and three participants in the steady-state task connectivity analysis $(F(1,7)>6.3, p<0.04)$, being non-significant during rest $(F(1,7)<0.4, p>0.55)$. The modulation of the task 
Table 2. Sensitivity and specificity in detecting bimanual tapping.

\begin{tabular}{|c|c|c|c|c|}
\hline & $\begin{array}{l}\text { Block activation-level } \\
\text { right M1 }\end{array}$ & $\begin{array}{l}\text { Block correlations 26-s full } \\
\text { task window }\end{array}$ & $\begin{array}{l}\text { Block correlations } 12-\mathrm{s} \\
\text { steady-state task window }\end{array}$ & $\begin{array}{l}\text { Block correlations 12-s } \\
\text { rest window }\end{array}$ \\
\hline Participant 01 & $86 \% / 91 \%^{*}$ & $94 \% / 88 \% *$ & $67 \% / 56 \%$ & $68 \% / 56 \%$ \\
\hline Participant 02 & $86 \% / 84 \% *$ & $94 \% / 97 \% *$ & $79 \% / 66 \% *$ & $75 \% / 47 \% *$ \\
\hline Participant 03 & $83 \% / 100 \% *$ & 96\%/97\%* & $63 \% / 63 \%^{*}$ & $49 \% / 47 \%$ \\
\hline Participant 04 & $85 \% / 94 \% *$ & $88 \% / 75 \% *$ & $69 \% / 53 \% *$ & $54 \% / 31 \%$ \\
\hline Participant 05 & $90 \% / 84 \% *$ & 92\%/97\%* & $64 \% / 59 \%$ & $66 \% / 63 \%$ \\
\hline Group & $86 \% / 91 \%$ & $93 \% / 91 \%$ & $68 \% / 59 \%$ & $62 \% / 49 \%$ \\
\hline
\end{tabular}

The sensitivity and specificity (sensitivity/specificity) in detecting if a task was performed with only the right index finger (unimanual), or with both index fingers (bimanual) was computed for all participants using a simple threshold approach (bold with asterisk = significant results).

connectivity by tapping speed was most pronounced when demand on bimanual coordination was high, and overall task difficulty was therefore increased. This effect was unique to the two sets of task connectivity measures, as the modulation of the activation level-based measures by tapping speed did not become more pronounced with increasing demand on bimanual coordination in both left and right primary motor cortex (figure 5, figure 7). Post hoc tests regarding the linear effect of tapping speed, performed separately for the three bimanual tapping tasks, confirmed this interaction effect (table 3, figure 7). The criterion validity of the correlation measures regarding tapping speed increases from synchronous tapping (steady-state $=-0.06 /$ overall $=$ 0.09), to alternating tapping (steady-state $=0.14 /$ overall $=0.29$ ), to bimanual unbalanced tapping (steady-state $=0.14$ / overall $=0.34$ ), while this effect was not found for the activation level-based measures (synchronous: 0.35, alternating: 0.36, unbalanced 0.30 , table 3 , figure 7). Overall, the task connectivity measures thus differentiate best regarding overall task difficulty, showing the strongest increase from low to high overall difficulty. A direct statistical comparison between the different sets of measures confirmed that both sets of activation level-based measures differed statistically from both sets of task connectivity measures (26-s full task window vs. right M1: interaction effect measure*bimanual coordination: Group $F(3,117)=22.5, p<$ 0.001 , significant in three participants, $F(3,21)>9.7, p<0.001 ; 26$-s full task window vs. left M1: interaction effect measure*bimanual coordination: Group $F(3,117)=23.9$, $p<0.001$, significant in four participants, $F(3,21)>15.8, p<0.001$; 26-s full task window vs. left M1: interaction effect measure*tapping speed: Group $F(3,117)=7.7, p$ $<0.001$, significant in four participants, $F(3,21)>3.1, p<0.04) ; 12-$ s full task window 
Table 3. Correlation with finger tapping speed.

\begin{tabular}{|c|c|c|c|c|}
\hline & $\begin{array}{l}\text { Block activation-level } \\
\text { left M1 }\end{array}$ & $\begin{array}{l}\text { Block correlations 26-s } \\
\text { full task window }\end{array}$ & $\begin{array}{l}\text { Block correlations } 12-s \\
\text { steady-state task window }\end{array}$ & $\begin{array}{l}\text { Block correlations 12-s } \\
\text { rest window }\end{array}$ \\
\hline \multicolumn{5}{|c|}{ Bimanual synchronous } \\
\hline Participant 01 & 0.26 & 0.26 & -0.01 & 0.15 \\
\hline Participant 02 & 0.18 & 0.03 & -0.11 & 0.16 \\
\hline Participant 03 & $0.61 *$ & 0.01 & 0.11 & 0.30 \\
\hline Participant 04 & 0.31 & 0.20 & -0.09 & -0.14 \\
\hline Participant 05 & $0.40 *$ & -0.03 & -0.21 & -0.18 \\
\hline Group & 0.35 & 0.09 & -0.06 & 0.06 \\
\hline \multicolumn{5}{|c|}{ Bimanual alternating } \\
\hline Participant 01 & $0.52^{*}$ & 0.24 & -0.21 & 0.30 \\
\hline Participant 02 & 0.05 & 0.03 & 0.07 & 0.06 \\
\hline Participant 03 & $0.62 *$ & 0.08 & 0.20 & 0.17 \\
\hline Participant 04 & 0.22 & $0.62^{*}$ & $0.43^{*}$ & 0.06 \\
\hline Participant 05 & $0.39 *$ & $0.47^{*}$ & 0.21 & 0.04 \\
\hline Group & 0.36 & 0.29 & 0.14 & 0.13 \\
\hline \multicolumn{5}{|c|}{ Bimanual unbalanced } \\
\hline Participant 01 & $0.39 *$ & $0.14 *$ & -0.11 & 0.01 \\
\hline Participant 02 & -0.20 & 0.34 & 0.15 & -0.04 \\
\hline Participant 03 & $0.74 *$ & $0.33^{*}$ & 0.01 & -0.01 \\
\hline Participant 04 & $0.43^{*}$ & $0.42^{*}$ & $0.47^{*}$ & 0.12 \\
\hline Participant 05 & 0.12 & $0.46^{*}$ & 0.20 & 0.04 \\
\hline Group & 0.30 & 0.34 & 0.14 & 0.03 \\
\hline \multicolumn{5}{|c|}{ Averaged across bimanual tapping tasks } \\
\hline Participant 01 & $0.34 *$ & 0.18 & -0.09 & 0.16 \\
\hline Participant 02 & 0.06 & 0.13 & 0.00 & 0.06 \\
\hline Participant 03 & $0.61^{*}$ & $0.17^{*}$ & 0.09 & 0.14 \\
\hline Participant 04 & $0.31 *$ & $0.38^{*}$ & $0.25^{*}$ & 0.00 \\
\hline Participant 05 & $0.30 *$ & $0.34 *$ & 0.06 & -0.02 \\
\hline Group & 0.32 & 0.24 & 0.06 & 0.07 \\
\hline
\end{tabular}

The criterion validity for detecting performed tapping speed was calculated by correlating the block-wise brain measures with the block-wise finger tapping speed. These brain-behavior correlations are presented for all participants for each of the three different bimanual tapping tasks separately as well as averaged (bold with asterisk = significant results).

vs. right $\mathrm{M} 1$ : interaction effect measure*bimanual coordination: Group $\mathrm{F}(3,117)=77.5$, $p<0.001$, significant in all participants, $F(3,21)>10.7, p<0.001 ; 12$-s full task window vs. left M1: interaction effect measure*bimanual coordination: Group $F(3,117)=14.6$, $p<0.001$, significant in three participants, $F(3,21)>15.8, p<0.008 ; 12$-s full task window vs. left M1: interaction effect measure*tapping speed: Group $F(3,117)=7.7, p$ $<0.001$, significant in three participants, $F(3,21)>5.0, p<0.009)$ ). 


\section{A \% signal change \\ B \\ Correlation 26-s task window \\ C \\ Correlation 12-s task window}

Bimanual synchronous
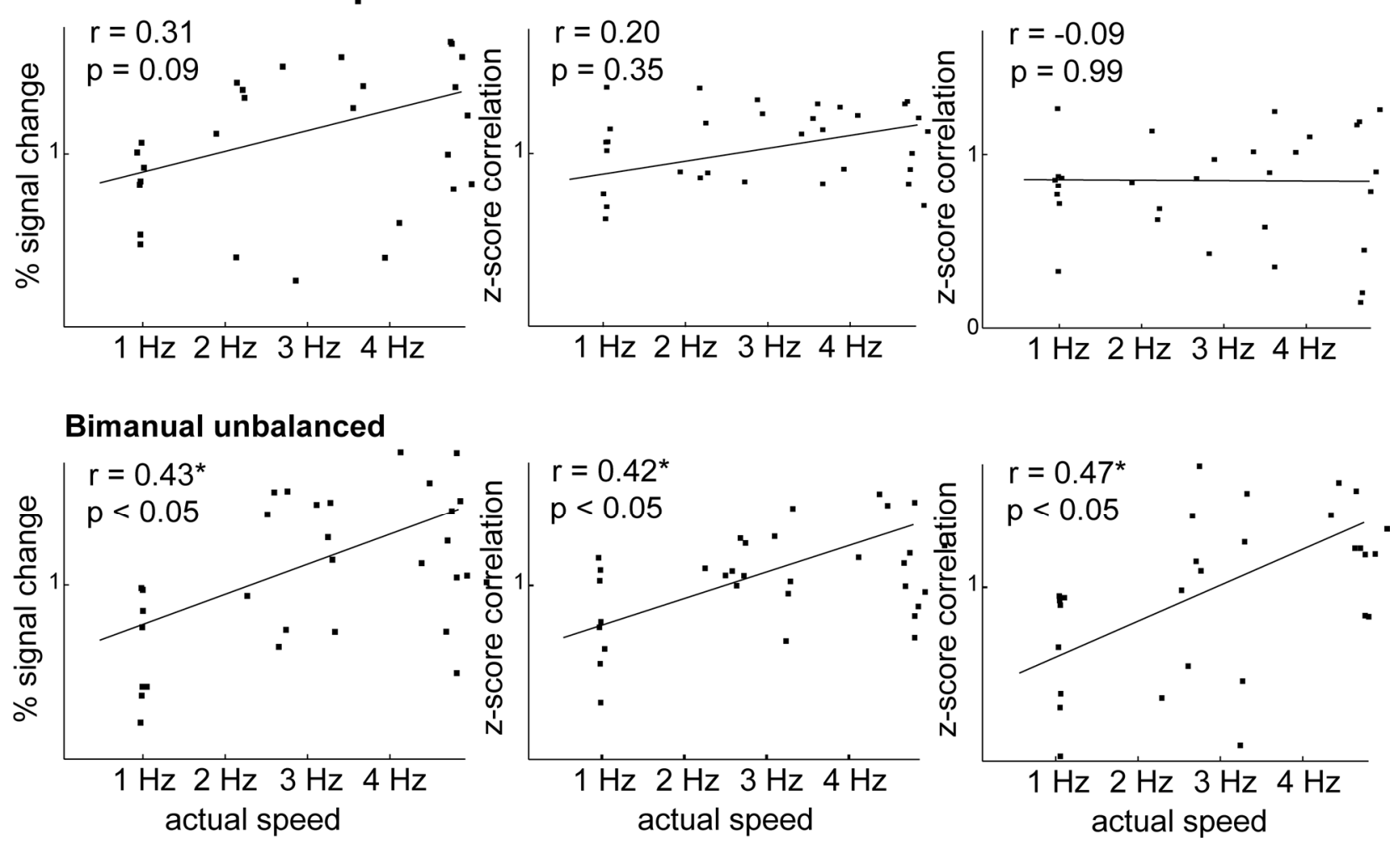

Figure 7. Correlation with finger tapping speed.

The criterion validity for detecting performed tapping speed was calculated by correlating the block-wise brain measures with the block-wise behavioral performance measures. The results from one representative participant are depicted for bimanual synchronous tapping (upper row) and bimanual unbalanced tapping (lower row). The correlation between finger tapping speed and the block-wise activation-level measures from left M1 (panel A), the 26-s full task blockwise correlations (panel B), and the 12-s steady-state task correlations (panel C) are shown. Each dot represents one block, with the regression line indicating the average strength of the brain-behavior correlation. Significant results are marked with an asterisk. The steady-state connectivity measures were modulated by finger tapping speed during the most difficult unbalanced tapping task, but not during the easier synchronous tapping task. The same effect is visible but less pronounced for the overall task connectivity measures, and much weaker for the activation-level based measures. The connectivity measures thus indicate overall task difficulty best, showing the strongest increase from low to high overall task difficulty. 


\section{Discussion}

The purpose of this study was to investigate whether windowed functional connectivity measures in comparison with activation level-based measures may be a good indicator of changes in task performance during a well-controlled, simple behavioral (here motor) task. This was assessed in a small number of subjects as a proof-of principle study using variants of a simple bimanual motor task at a single-trial level to investigate the feasibility of windowed correlations as a (potential) neurofeedback signal. We observed four main findings: first, windowed correlations computed based on very short time windows did indeed provide valid information on certain task aspects. Second, the obtained information was unique, as task connectivity measures were more indicative of overall task difficulty than activation level-based measures. Third, the robustness of the steady-state task connectivity measures with the chosen approach was relatively low, and fourth, the task dependent modulation of functional connectivity was spatially focused within the task-relevant network.

Two different sorts of task connectivity measures were investigated: steady-state task connectivity as an index of integration during continuous performance, and overall task connectivity as a compound measure indexing both steady-state performance and gross activation level changes. For both sets of task connectivity measures significant brain-behavior relationships were found, which were unique in comparison with activation-level based measures. While we hypothesized that functional connectivity measures may be more sensitive to bimanual coordination demands, and less to finger tapping speed, we found that they were most indicative of overall task difficulty. While activation-level based measures increased with increasing finger tapping speed, and decreased with increasing demand on bimanual coordination, steady-state task connectivity increased with increasing tapping speed, as well as with increasing demand on bimanual coordination. The highest activation levels were thus found for high speed tapping during an easy tapping sequence, while steady-state connectivity was highest when overall task difficulty was high. The full task window correlations, a compound measure between activation level changes and steady-state, showed a mix of those two effects. In general, the two sorts of task connectivity measures thus indexed overall task difficulty better than activation level-based measures. While these results need to be interpreted with caution due to the small sample size, and do not allow for population inference, they were consistent across participants. 
The findings are in line with previous studies showing that increasing demand on bimanual coordination, or tapping speed during finger tapping leads to higher functional connectivity $[36,37]$. The presented findings furthermore corroborate previous research showing that an increasing cognitive demand during the performance of working memory tasks also enhances functional connectivity between task-relevant regions $[46,47,48,49]$. Finally, the presented findings are consistent with previous imaging studies showing that task-dependent enhanced interhemispheric coupling is highest during early stages of motor skill learning, when task difficulty is highest [41]. Three recent studies on motor learning showed that the functional connectivity within the motor network rapidly increased during an initial stage of learning, and then decreased as learning slowed down and performance stabilized $[50,51,52]$. The results from the presented study, as well as from previous studies thus support the idea that functional connectivity measures may be used as an indicator of overall task difficulty during neurofeedback training. The presented study extends previous findings by showing that short-window correlations can capture this task aspect, and seem to be more indicative than activation level-based measures.

A third finding of this study was the low robustness of the steady-state task connectivity measures. While the steady-state correlations seem to have potential for indexing task aspects, which cannot be captured equally well by activation level-based measures, their reliability was considerably lower. Effect sizes were relatively small and the consistency of the results across participants thus compromised. Further research into improving the stability of steady-state connectivity measures thus seems necessary to make a short-window connectivity-based neurofeedback training implementation feasible. To optimize the data quality through dense sampling we used a relatively high temporal resolution in comparison with standard imaging parameters, compromising on spatial coverage in return. At the same time we attempted to maximize the signal to noise ratio through the use of relatively large functional voxels. However, further optimization of the data quality seems crucial in order to provide robust measures. Further future improvements could be achieved by further reduction of spatial coverage, fundamentally improved hardware, advanced imaging sequences [53,54], new methods for noise reduction [55], or noise removal [56].

Finally, the network analysis performed on the group-level showed that the taskdependent modulation of the functional connectivity was clearly spatially focused within the analyzed task network. Similar results have been reported by other studies, 
showing that task-dependent modulations of brain connectivity patterns are often spatially quite restricted $[57,58]$. Therefore, the use of bivariate pairwise correlations as a measure of task-dependent modulations in functional networks might be a promising approach for a functional connectivity neurofeedback implementation. The advantage of this approach might be that it would allow for further optimization of the imaging sequence parameters by limiting spatial coverage and increasing temporal resolution. More research will be necessary to confirm if this significantly improves the robustness and sensitivity of the functional connectivity measures.

Overall, the presented results thus support the idea that functional connectivity measures may be valuable indicators of task difficulty for neurofeedback based learning. Functional connectivity neurofeedback could provide relevant, and to a certain extent unique information during neurofeedback training. Windowed correlations may serve as an indicator of overall task difficulty on an individual level, indicating how difficult a task is for this individual at this moment in time. Overall, functional connectivity measures may thus add an important estimate regarding the individual learning process in comparison with the activation level-based feedback measures as previously used in neurofeedback patient training studies [59,60,61,62], and in multivariate real-time approaches currently under investigation [63]. Further research into the generalizability of the results to other task paradigms and patient populations thus seems worth pursuing.

\section{Conclusions}

The present study set out to investigate the general feasibility of fMRI connectivitybased neurofeedback. Our results demonstrate that task connectivity seems to provide unique information on task difficulty. If functional connectivity measures can provide a valid index of individual task difficulty during learning, this might be extremely valuable for patients. During training, especially in a patient setup, participants are often encouraged to adopt and employ novel cognitive, behavioral and emotional strategies. An individual index of task difficulty could encourage patients to constantly perform at a high level of individual difficulty, something that may necessary for mastering the novel cognitive, behavioral and emotional skills, which patients are lacking. If the results of this study could be generalized, windowed functional connectivity 
70 | CHAPTER 2

neurofeedback may therefore indeed become a valuable additional tool for neurofeedback training setups.

\section{Acknowledgements}

We thank A. Heinecke and J. Eck for their helpful suggestions regarding the analysis, and V. Kemper for his support with optimizing fMRI data acquisition and preprocessing. The authors gratefully acknowledge the support of the BrainGain Smart Mix Program of The Netherlands Ministry of Economic Affairs and The Netherlands Ministry of Education, Culture and Science (grant number: SSM06011). 


\section{References}

1. Goebel R, Zilverstand A, Sorger B (2010) Real-time fMRI-based brain-computer interfacing for neurofeedback therapy and compensation of lost motor functions. Imaging in Medicine 2: 407-415.

2. Weiskopf, N. (2012). Real-time fMRI and its application to neurofeedback. Neurolmage, 62(2), 682-92.

3. Sulzer J, Haller S, Scharnowski F, Weiskopf N, Birbaumer N, et al. (2013) Real-time fMRI neurofeedback: Progress and challenges. Neuroimage 76: 386-399.

4. Bullmore $E$ (2012) The future of functional MRI in clinical medicine. Neuroimage 62: 1267-1271.

5. Konrad K, Eickhoff SB (2010) Is the ADHD brain wired differently? A review on structural and functional connectivity in attention deficit hyperactivity disorder. Hum Brain Mapp 31: 904-916.

6. Stephan KE, Baldeweg T, Friston KJ (2006) Synaptic plasticity and dysconnection in schizophrenia. Biol Psychiatry 59: 929-939.

7. Minshew NJ, Keller TA (2010) The nature of brain dysfunction in autism: functional brain imaging studies. Curr Opin Neurol 23: 124-130.

8. Kim MJ, Loucks RA, Palmer AL, Brown AC, Solomon KM, et al. (2011) The structural and functional connectivity of the amygdala: from normal emotion to pathological anxiety. Behav Brain Res 223: 403-410.

9. Pizzagalli DA (2011) Frontocingulate dysfunction in depression: toward biomarkers of treatment response. Neuropsychopharmacology 36: 183-206.

10. Poston KL, Eidelberg D (2012) Functional brain networks and abnormal connectivity in the movement disorders. Neuroimage 62: 2261-2270.

11. Rubia K, Halari R, Cubillo A, Mohammad AM, Brammer M, et al. (2009) Methylphenidate normalises activation and functional connectivity deficits in attention and motivation networks in medication-naive children with ADHD during a rewarded continuous performance task. Neuropharmacology 57: 640652.

12. Anand A, Li Y, Wang Y, Wu J, Gao S, et al. (2005) Antidepressant effect on connectivity of the mood-regulating circuit: an FMRI study. Neuropsychopharmacology 30: 1334-1344.

13. Anand A, Li Y, Wang Y, Gardner K, Lowe MJ (2007) Reciprocal effects of antidepressant treatment on activity and connectivity of the mood regulating circuit: an FMRI study. J Neuropsychiatry Clin Neurosci 19: 274-282.

14. Weiskopf N, Scharnowski F, Veit R, Goebel R, Birbaumer N, et al. (2004) Selfregulation of local brain activity using real-time functional magnetic resonance imaging (fMRI). J Physiol Paris 98: 357-373.

15. Sitaram R, Caria A, Veit R, Gaber T, Rota G, et al. (2007) FMRI brain-computer interface: a tool for neuroscientific research and treatment. Comput Intell Neurosci: 25487. 
16. Ruiz S, Birbaumer N, Sitaram R (2013) Abnormal neural connectivity in schizophrenia and fMRI-brain-computer interface as a potential therapeutic approach. Front Psychiatry 4: 17.

17. Koush Y, Rosa MJ, Robineau F, Heinen K, S WR, et al. (2013) Connectivity-based neurofeedback: Dynamic causal modeling for real-time fMRI. Neuroimage 81C: 422-430.

18. Friston KJ (2011) Functional and effective connectivity: a review. Brain Connect 1: 13-36.

19. Rogers BP, Morgan VL, Newton AT, Gore JC (2007) Assessing functional connectivity in the human brain by fMRI. Magn Reson Imaging 25: 1347-1357.

20. Smith SM (2012) The future of FMRI connectivity. Neuroimage 62: 1257-1266.

21. Smith SM, Miller KL, Salimi-Khorshidi G, Webster M, Beckmann CF, et al. (2011) Network modelling methods for FMRI. Neuroimage 54: 875-891.

22. Joel SE, Caffo BS, van Zijl PC, Pekar JJ (2011) On the relationship between seedbased and ICA-based measures of functional connectivity. Magn Reson Med 66: 644-657.

23. Posse S, Binkofski F, Schneider F, Gembris D, Frings W, et al. (2001) A new approach to measure single-event related brain activity using real-time fMRI: feasibility of sensory, motor, and higher cognitive tasks. Hum Brain Mapp 12: 25-41.

24. Esposito F, Seifritz E, Formisano E, Morrone R, Scarabino T, et al. (2003) Realtime independent component analysis of fMRI time-series. Neuroimage 20: 2209-2224.

25. Sorger B, Reithler J, Dahmen B, Goebel R (2012) A real-time fMRI-based spelling device immediately enabling robust motor-independent communication. Curr Biol 22: 1333-1338.

26. Birn RM, Murphy K, Handwerker DA, Bandettini PA (2009) fMRI in the presence of task-correlated breathing variations. Neuroimage 47: 1092-1104.

27. Birn RM (2012) The role of physiological noise in resting-state functional connectivity. Neuroimage 62: 864-870.

28. Weissenbacher A, Kasess C, Gerstl F, Lanzenberger R, Moser E, et al. (2009) Correlations and anticorrelations in resting-state functional connectivity MRI: a quantitative comparison of preprocessing strategies. Neuroimage 47: 14081416.

29. Jo HJ, Saad ZS, Simmons WK, Milbury LA, Cox RW (2010) Mapping sources of correlation in resting state FMRI, with artifact detection and removal. Neuroimage 52: 571-582.

30. Hutton C, Josephs O, Stadler J, Featherstone E, Reid A, et al. (2011) The impact of physiological noise correction on fMRI at $7 \mathrm{~T}$. Neuroimage 57: 101-112.

31. Guo CC, Kurth F, Zhou J, Mayer EA, Eickhoff SB, et al. (2012) One-year test-retest reliability of intrinsic connectivity network fMRI in older adults. Neuroimage 61: 1471-1483. 
32. Rao SM, Bandettini PA, Binder JR, Bobholz JA, Hammeke TA, et al. (1996) Relationship between finger movement rate and functional magnetic resonance signal change in human primary motor cortex. J Cereb Blood Flow Metab 16: 1250-1254.

33. Riecker A, Wildgruber D, Mathiak K, Grodd W, Ackermann H (2003) Parametric analysis of rate-dependent hemodynamic response functions of cortical and subcortical brain structures during auditorily cued finger tapping: a fMRI study. Neuroimage 18: 731-739.

34. Swinnen SP, Wenderoth N (2004) Two hands, one brain: cognitive neuroscience of bimanual skill. Trends Cogn Sci 8: 18-25.

35. Biswal B, Yetkin FZ, Haughton VM, Hyde JS (1995) Functional connectivity in the motor cortex of resting human brain using echo-planar MRI. Magn Reson Med 34: 537-541.

36. Sun FT, Miller LM, D'Esposito M (2004) Measuring interregional functional connectivity using coherence and partial coherence analyses of fMRI data. Neuroimage 21: 647-658.

37. Newton AT, Morgan VL, Gore JC (2007) Task demand modulation of steady-state functional connectivity to primary motor cortex. Hum Brain Mapp 28: 663-672.

38. Fall S, de Marco G (2008) Assessment of brain interactivity in the motor cortex from the concept of functional connectivity and spectral analysis of fMRI data. Biol Cybern 98: 101-114.

39. Rogers BP, Gore JC (2008) Empirical comparison of sources of variation for FMRI connectivity analysis. PLoS One 3: e3708.

40. Hampson M, Olson IR, Leung HC, Skudlarski P, Gore JC (2004) Changes in functional connectivity of human MT/V5 with visual motion input. Neuroreport 15: $1315-1319$.

41. Sun FT, Miller LM, Rao AA, D'Esposito M (2007) Functional connectivity of cortical networks involved in bimanual motor sequence learning. Cereb Cortex 17: 1227-1234.

42. Oldfield RC (1971) The assessment and analysis of handedness: the Edinburgh inventory. Neuropsychologia 9: 97-113.

43. Talairach G, Tournoux $P$ (1988) Co-planar stereotaxic atlas of the human brain. New York: Thieme.

44. Witt ST, Laird AR, Meyerand ME (2008) Functional neuroimaging correlates of finger-tapping task variations: an ALE meta-analysis. Neuroimage 42: 343-356.

45. Fischer RA (1928) The General Sampling Distribution of the Multiple Correlation Coefficient. Proceedings of the Royal Society of London Series A, Containing Papers Mathematical Physical Character 121: 654-673.

46. Engstrom M, Landtblom AM, Karlsson T (2013) Brain and effort: brain activation and effort-related working memory in healthy participants and patients with working memory deficits. Front Hum Neurosci 7: 140.

47. Nagel IE, Preuschhof C, Li SC, Nyberg L, Backman L, et al. (2011) Load modulation of BOLD response and connectivity predicts working memory performance in younger and older adults. J Cogn Neurosci 23: 2030-2045. 
48. Leech R, Kamourieh S, Beckmann CF, Sharp DJ (2011) Fractionating the default mode network: distinct contributions of the ventral and dorsal posterior cingulate cortex to cognitive control. J Neurosci 31: 3217-3224.

49. Fu CH, Mclntosh AR, Kim J, Chau W, Bullmore ET, et al. (2006) Modulation of effective connectivity by cognitive demand in phonological verbal fluency. Neuroimage 30: 266-271.

50. Yoo K, Sohn WS, Jeong Y (2013) Tool-use practice induces changes in intrinsic functional connectivity of parietal areas. Front Hum Neurosci 7: 49.

51. Coynel D, Marrelec G, Perlbarg V, Pelegrini-Issac M, Van de Moortele PF, et al. (2010) Dynamics of motor-related functional integration during motor sequence learning. Neuroimage 49: 759-766.

52. Ma L, Narayana S, Robin DA, Fox PT, Xiong J (2011) Changes occur in resting state network of motor system during 4 weeks of motor skill learning. Neuroimage 58: 226-233.

53. Feinberg DA, Moeller, S, Smith, SM, Auerbach E, Ramanna S, et al. (2010) Multiplexed Echo Planar Imaging for Sub-Second Whole Brain FMRI and Fast Diffusion Imaging. PLoS One 5: e15710.

54. Posse S, Ackley E, Mutihac R, Zhang T, Hummatov R et al. (2013) High-speed real-time resting-stte fMRI using multi-slab echo-volumar imaging. Front Hum Neurosci 7: 479.

55. Zaitsev M, Dold C, Sakas G, Hennig J, Speck O (2006) Magnetic resonance imaging of freely moving objects: prospective real-time motion correction using an external optical motion tracking system. Neuroimage 31: 1038-1050.

56. Koush Y, Zvyagintsev M, Dyck M, Mathiak KA, Mathiak K (2012) Signal quality and Bayesian signal processing in neurofeedback based on real-time fMRI. Neuroimage 59: 478-489.

57. Jiang $T$, He $Y$, Zang $Y$, Weng X (2004) Modulation of functional connectivity during the resting state and the motor task. Hum Brain Mapp 22: 63-71.

58. Grefkes C, Eickhoff SB, Nowak DA, Dafotakis M, Fink GR (2008) Dynamic intraand interhemispheric interactions during unilateral and bilateral hand movements assessed with fMRI and DCM. Neuroimage 41: 1382-1394.

59. deCharms RC, Maeda F, Glover GH, Ludlow D, Pauly JM, et al. (2005) Control over brain activation and pain learned by using real-time functional MRI. Proc Natl Acad Sci USA 102: 18626-18631.

60. Haller S, Birbaumer N, Veit R (2010) Real-time fMRI feedback training may improve chronic tinnitus. Eur Radiol 20: 696-703.

61. Subramanian L, Hindle JV, Johnston S, Roberts MV, Husain M, et al. (2011) Realtime functional magnetic resonance imaging neurofeedback for treatment of Parkinson's disease. J Neurosci 31: 16309-16317.

62. Linden DE, Habes I, Johnston SJ, Linden S, Tatineni R, et al. (2012) Real-time self-regulation of emotion networks in patients with depression. PLoS One 7: e38115.

63. LaConte SM (2011) Decoding fMRI brain states in real-time. Neuroimage 56: 440454. 


\section{Measuring anxiety based on the brain:}

"Quantitative representations of subjective anxiety levels in the brain. A parametric fMRI study in spider phobia"

Based on: Zilverstand, A., Sorger, B., Kaemingk, A., \& Goebel, R. Quantitative representations of subjective anxiety levels in the brain. A parametric fMRI study in spider phobia.

[in preparation]. 


\section{Abstract}

Affective neuroscience research can potentially guide the development of new therapeutic approaches by enhancing our understanding of brain processing in mental disorders. We employed a parametric fMRI anxiety provocation design in healthy and spider phobic participants to gain a better understanding of the functional role of brain regions implicated in anxiety disorders. This design allowed us to tease apart which brain regions are genuinely involved in the evaluation of threat, which represent subjective anxiety levels, and which support secondary cognitive processes, such as goal setting. In order to provoke different levels of anxiety, we created a parametric spider picture set, by manipulating perceived proximity to spiders. A behavioral stimulus evaluation study showed that subjective anxiety increased linearly with increasing proximity. We employed the parametric stimuli in an fMRI anxiety provocation study, conducting a whole-brain categorical and parametric GLM analysis. The expected anxiety network could be mapped with both analysis approaches. Based on the parametric analysis of the data we were able to distinguish three processing levels: (1) an lower processing level corresponding mainly to initial threat monitoring, encompassing visual areas, the thalamus, amygdala, and anterior insula, (2) an intermediate processing level, at which subjective levels of anxiety are reflected, including the mid insula, and the dorsolateral anterior cingulate, and (3) a higher processing level, at which secondary cognitive processes are supported, comprising an active prefrontal network. We conclude, that in spider phobia anxiety processing appears to be organized hierarchically, that parametric fMRI designs can be used to tease apart the relevant processing levels, and that induced anxiety levels are indeed represented quantitatively in the brain. 


\section{Introduction}

How does the brain represent subjective levels of anxiety, and is it possible to measure anxiety in patients based on the functional magnetic resonance imaging (fMRI) blood oxygenation level-dependent (BOLD) signal? Until now, there is no conclusive evidence demonstrating whether particular brain regions contain a quantitative representation of subjective anxiety levels in patients. This question is of decisive importance, as a thorough understanding of how the brain represents complex healthy and pathological mental states could potentially inform the development, evaluation, and optimization of new therapeutic approaches. Currently, about $12-18 \%$ of the adult population suffer from an anxiety disorder (Kessler, Chiu, Demler, Merikangas, \& Walters, 2005; Wittchen \& Jacobi, 2005), while treatment success rates for anxiety patients have stagnated during the last 40 years (Ost, 2008). Previous research with $\mathrm{fMRI}$ has shown that psychotherapy indeed induces measurable changes in brain processing (Straube, Glauer, Dilger, Mentzel, \& Miltner, 2006), however, a good understanding of the induced changes is necessary for a clear interpretation. A better understanding of the functional role of particular brain regions could inform the development of new therapeutic approaches, by monitoring changes induced by medication-based treatments, and new psychotherapeutic approaches. It could also provide relevant knowledge for the development of therapeutic procedures aimed at targeting particular brain regions directly, by stimulation or self-regulation. One tool for providing a richer characterization of brain responses are parametric fMRI designs, which can tease apart categorical on-off responses from responses which are quantitatively related to the factor which is being manipulated. This approach allows to separate brain regions genuinely involved in evaluating and representing the manipulated factor, in this case anxiety level, from regions supporting secondary cognitive processes such as the formation of memory, representation of context information, and goal setting.

Parametric designs have been used early on in $\mathrm{fMRI}$ research in order to show that the blood oxygenation level-dependent (BOLD) response in primary visual areas increases linearly with relevant stimulus properties such as stimulus frequency (Kwong et al., 1992), while it depends on motor aspects such as finger movement rate in primary motor cortex (Rao et al., 1996). It was also shown that while a graded response related to pain stimulus intensity can be detected in somatosensory cortex, as well as 
the anterior cingulate cortex, only the anterior cingulate cortex response reflected the level at which pain was perceived subjectively (Helmchen, Mohr, Erdmann, Binkofski, \& Büchel, 2006; Mohr, Binkofski, Erdmann, Büchel, \& Helmchen, 2005; Rainville, 1997). Furthermore, the response in both anterior cingulate and somatosensory cortex was linearly related to anticipated pain intensity when participants were awaiting a strong electrical shock (Straube, Schmidt, Weiss, Mentzel, \& Miltner, 2009). In studies on higher cognitive functions parameters, task difficulty was manipulated to show that the modulation of the BOLD response in a prefrontal-parietal network was linearly related to task load (Jansma, Ramsey, Coppola, \& Kahn, 2000). Finally, research in affective neuroscience demonstrated that medial brain regions, as orbito- and dorsomedial prefontral cortex, medial parietal cortex, and the insula, represent emotional valence quantitatively (Heinzel et al., 2005).

More recently, the neural systems involved in monitoring threat evoked by a tarantula spider have been investigated in healthy participants with low to medium spider fear using a parametric design (Mobbs et al., 2010). Participants were made to believe that a living tarantula spider was placed next to their foot in the fMRI scanner at varying distances, either in approaching or retreating order, to isolate the neural networks that encode proximity, approach, and subjective estimation of threat. The detected network representing proximity encompassed the midbrain, amygdala, striatum, insula and dorsal anterior cingulate cortex, but only the activation level of the amygdala, striatum and insula represented if the spider was approaching rather than retracting, and only the amygdala response was correlated with subjectively perceive threat (Mobbs et al., 2010). The authors concluded that in healthy participants the amygdala has a key role in threat monitoring in an ecologically valid context (Mobbs et al., 2010). However, as the participants had spider fear significantly below the fear level of spider phobics, the results cannot be directly generalized to patients. Therefore, the only parametric fMRI study conducted with anxiety patients so far, is a study investigating if low, moderate and high intensity threatening faces would provoke different levels of BOLD response in the amygdala in social phobics (Klumpp, Angstadt, Nathan, \& Phan, 2010). The region of interest analysis showed that the amygdala response of social phobics depended on provoked fear level (Klumpp et al., 2010), but did not provide any information on other brain regions. A whole brain study investigating the parametric modulation of the BOLD response by subjective anxiety in patients has therefore not been conducted yet. 
The core anxiety network in patients has thus not been defined by disentangling regions involved in threat monitoring and representation of anxiety levels. What has been analyzed is which regions are commonly activated during symptom provocation in populations with different anxiety disorders, as well as in healthy subjects during fear conditioning. A meta-analysis following this approach, was performed on data from patients with specific phobia, social anxiety disorder, post-traumatic stress disorder, and healthy fear-conditioned participants. Two brain regions emerged, the amygdala and insula, which were consistently activated during anxiety provocation in all groups (Etkin \& Wager, 2007). The authors concluded that amygdala and insula seem to be core regions of a commonly shared neurobiological anxiety network (Etkin \& Wager, 2007). Brain regions that were most often co-activated, were the anterior cingulate, orbitofrontal cortex and thalamus (Etkin \& Wager, 2007). Other brain regions generally found to be activated in fMRI symptom provocation studies using visual stimulation, were visual areas, and in some studies the dorsolateral prefrontal cortex (Del Casale et al., 2012)

In order to investigate more directly, which brain regions evaluate and represent threat level in healthy versus spider phobic participants, we created and behaviorally validated a parametric spider picture set, for a fMRI anxiety provocation study. Photographs of spiders were manipulated regarding the experienced proximity of the viewer to the threatening animal. It is well known from exposure therapy that reducing distance to the animal will increase fear (Barlow, 2002). To ensure that the manipulation worked as hypothesized the stimulus material was rated by a group of low- and high-fear participants prior to the fMRI study. The stimuli were then employed in a $\mathrm{fMRI}$ symptom provocation study to examine which brain regions BOLD response would be modulated quantitatively dependent on provoked anxiety level.

We expected activation along the visual stream, as well as in the brain regions most often activated in symptom provocation studies, such as the amygdala, insula, anterior cingulate, and thalamus (Del Casale et al., 2012; Dilger et al., 2003; Etkin \& Wager, 2007; Goossens, Schruers, Peeters, Griez, \& Sunaert, 2007; Schienle, Schäfer, Walter, Stark, \& Vaitl, 2005; Veltman et al., 2004), and in frontal brain regions supporting cognitive processes, such as the orbitofrontal and dorsolateral prefrontal cortex (figure 1). We hypothesized that brain regions involved in threat monitoring would show a linear increase of BOLD signal with increasing perceived proximity in both the high- and low-fear group, while regions involved in the representation of 
anxiety would show a linear increase of activation levels in the high-fear group only. Finally, regions supporting secondary cognitive processes should not exhibit a parametric modulation by anxiety.

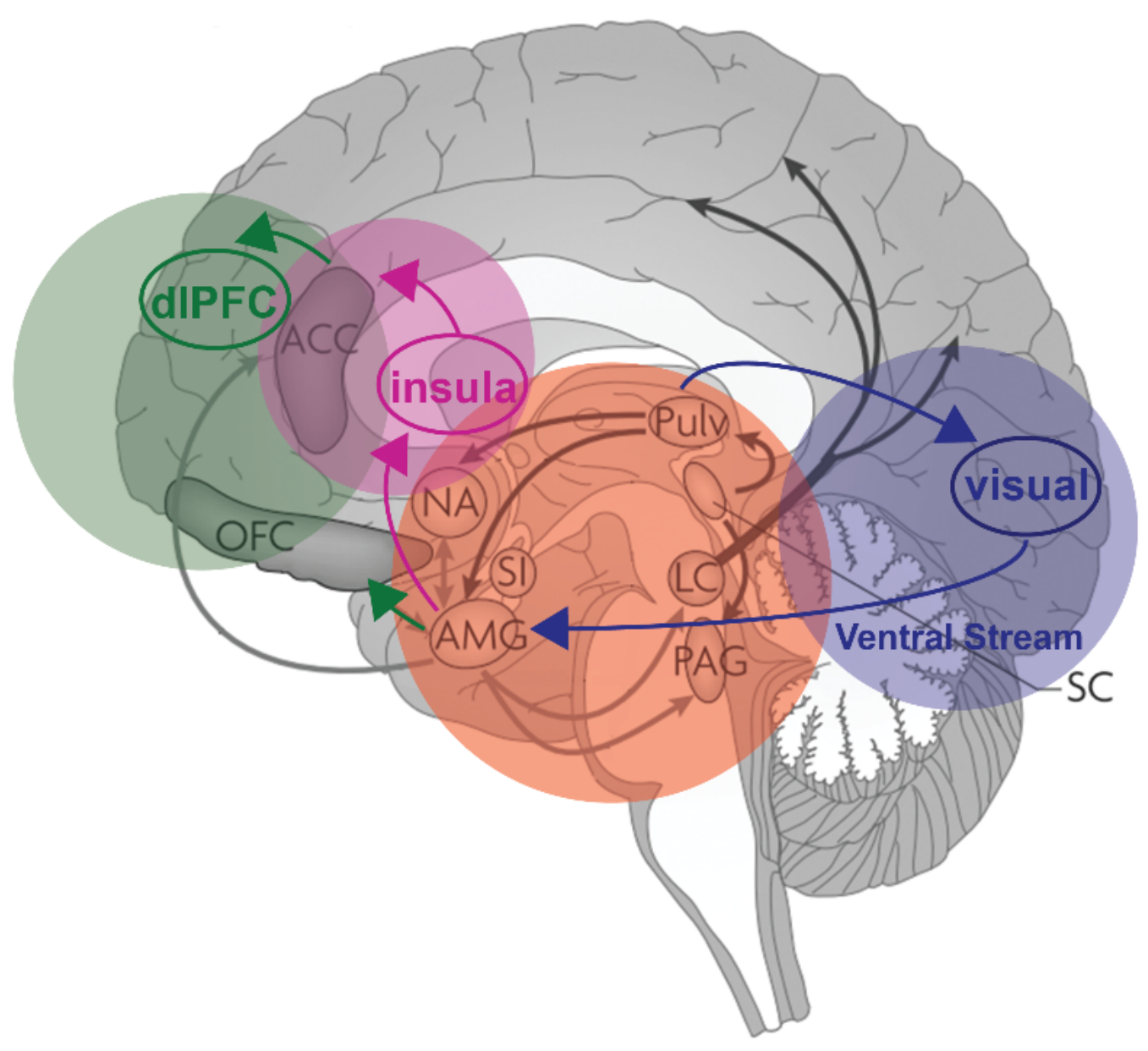

Figure 1. Brain regions involved during visual anxiety provocation.

Visual information is first processed in subcortical structures (orange), and the ventral and dorsal visual stream (only the ventral stream is shown in blue). During anxiety provocation medial-temporal (pink), and prefrontal (green) cortical areas are additionally activated. The figure was adapted with kind permission from Tamietto and de Gelder (2010).

AMG = amygdala, $\mathrm{SI}=$ substantia innominata, $\mathrm{NA}=$ nucleus accumbens, Pulv = pulvinar thalamus, $\mathrm{SC}=$ superior colliculus, $\mathrm{LC}=$ locus coeruleus, $\mathrm{PAG}=$ periaqueductal grey, $\mathrm{ACC}=$ anterior cingulate cortex, dIPFC = dorsolateral prefrontal cortex, $\mathrm{OFC}=$ orbitofrontal cortex . 


\section{Methods}

\section{Participants and Ethics Statement}

Participants were compromised of twenty-seven female high-fear [Spider Phobia Questionnaire (SPQ) Score $\geq 14$, (Klorman, Weerts, Hastings, Melamed, \& Lang, 1974)], and twenty-seven age-matched female low-fear (SPQ Score $\leq 4$ ) volunteers, who were recruited by public advertisement at Maastricht University. Forty of them participated in a behavioral rating study to evaluate the stimuli (table 1), and fourteen volunteers participated in the fMRI anxiety provocation study (table 2). All fMRI participants were additionally administered the Fear of Spider Questionnaire [FSQ

\begin{tabular}{l|l|l|l}
\hline \multicolumn{4}{l}{ Table 1. Participants stimulus rating } \\
$\begin{array}{l}\text { Variables (Mean } \\
\text { +l- SD) }\end{array}$ & High fear & Low fear & p-value \\
\hline Gender (female) & $\mathrm{n}=20$ & $\mathrm{n}=20$ & \\
\hline Age & $22.6(3.2)$ & $22.5(3.3)$ & $\mathrm{p}=0.88$ \\
\hline SPQ & $20.3(3.4)$ & $1.8(1.3)$ & $<0.001$ \\
\hline
\end{tabular}

Twenty high-fear, and twenty age-matched low-fear participants took part in a behavioral rating study to evaluate the stimuli. They were included based on their Spider Phobia Questionnaire (SPQ) score, and either belonged to the highest or lowest quartile of the population regarding spider fear (high fear $\geq 14$, low fear $\leq 4$ ).

\begin{tabular}{l|llll}
\hline \multicolumn{4}{l}{ Table 2. Participants fMRI study } \\
$\begin{array}{l}\text { Variables (Mean } \\
\text { +l- SD) }\end{array}$ & High fear & Low fear & p-value \\
\hline Gender (female) & $\mathrm{n}=7$ & $\mathrm{n}=7$ & \\
\hline Age & $21.7(3.9)$ & $20.9(2.2)$ & 0.63 \\
\hline SPQ & $21.0(2.9)$ & $2.6(1.8)$ & $<0.001$ \\
\hline FSQ & $88.7(14.0)$ & $5.7(9.6)$ & $<0.001$ \\
\hline SCID & $7.3(1.2)$ & $0.7(0.6)$ & $<0.001$ \\
\hline Duration (years) & $14.9(2.5)$ & & \\
\hline
\end{tabular}

Seven high-fear, and seven age-matched low-fear participants took part in the fMRI anxiety provocation study. They were included based on their SPQ score (high fear $\geq 14$, low fear $\leq$ 4), and additionally administered the Fear of Spider Questionnaire (FSQ) and Structured Clinical Interview DSM IV (SCID). The duration (years) since onset of spider phobia symptoms is noted. 
(Szymanski, 1995)], and diagnosed with spider phobia according to the criteria of The Diagnostic and Statistic Manual of Mental Disorders DSM-IV TR (American Psychiatric Association, 2000). All were free of psychotropic medication and were not affected by current or previous other neuropsychiatric comorbidity as evaluated by means of a structured clinical interview [Mini International Neuropsychiatric Interview, MINI, (Sheehan et al., 1998)]. Participants received a small financial compensation (8 $€ /$ hour), and gave their written informed consent prior to the experiment that was conducted in conformity with the Declaration of Helsinki and approved by the local Medical Ethics Committee at Maastricht University.

\section{Stimuli}

A set of 48 photo stimuli depicting European Spiders in their natural environment was acquired from the photographer Ed Nieuwenhuys (http://ednieuw.home.xs4all.nl/), here named natural spider picture set. Three selected spiders from this set were used to create a parametric spider picture set. First, the size and posture of the spiders were varied, such that the spider was presented in four different sizes (covering 1, 3, 5, or 7 degree visual angle at picture size $600 \times 600$ pixels), while at the same time its posture was changed such that it became more oriented towards the viewer (zoom dimension). Then, each of the manipulated spiders was mounted on variants of four different sorts of photographic backgrounds (1. nature, 2. computer keyboard, 3. clothes, 4. face). Through this manipulation the proximity of the spiders to the viewer was changed by its context (context dimension). As the experienced proximity to the animal gradually increased along both dimensions, both manipulations were hypothesized to continuously intensify anxiety in spider phobics (figure 2). All manipulations were carried out in Adobe Photoshop (Adobe Systems Inc., San Jose, U.S.A.) by a professional graphic designer using tools such as shadow cast in order to ensure that pictures appeared photo-realistic. As sixteen variants of each of the three selected spiders were created, the original parametric spider picture set used in the stimulus rating study encompassed 48 pictures. For the fMRI study, this original parametric spider picture set was further extended by adding variants of three additional spiders, and combining them with new backgrounds variants in order to generate a total number of 192 spider pictures following the principles described above. Finally, a neutral category with 48 pictures depicting background variants without spiders was added, so that the final parametric spider picture set encompassed 240 pictures. 


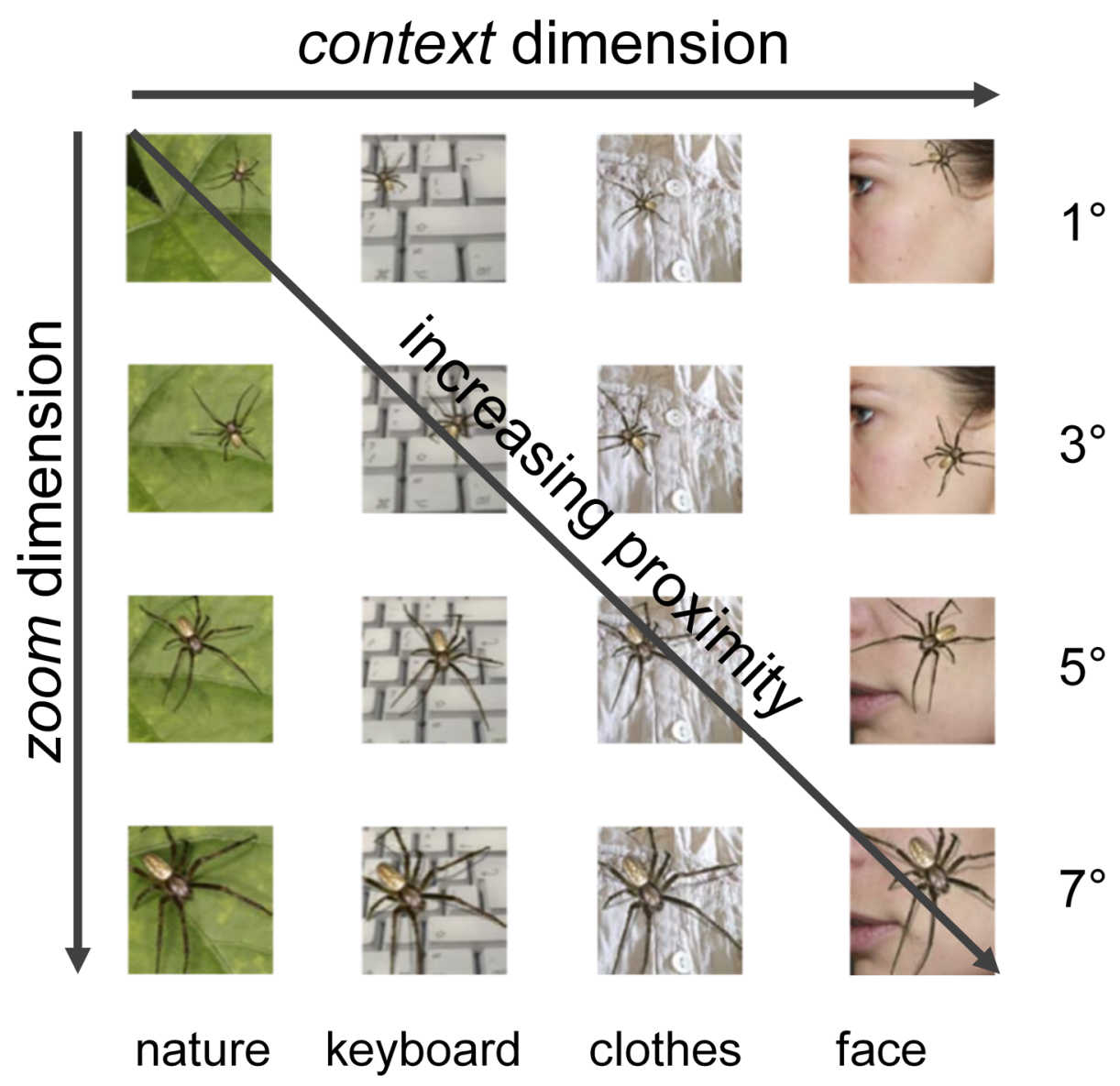

Figure 2. Stimulus dimensions.

Exemplary stimuli from the parametric spider picture set are presented. The stimuli were varied systematically along two dimensions. Along the zoom dimension the spider increased in size $(1,3,5$, or 7 degree visual angle for the largest presented picture size), and became more oriented towards the viewer. Along the context dimension the spider was placed in different contexts, increasing proximity by its context (1. nature, 2. computer keyboard, 3. clothes, 4. face). Both manipulations were hypothesized to gradually intensify provoked anxiety in spider phobics.

\section{Procedure stimulus rating}

The 48-picture parametric spider picture set, and the 48-picture natural spider picture set were shuffled, and presented in random order to each participant on a computer, with the experimenter present in an adjacent room. In order to compare the two picture sets a third manipulation of perceived proximity was added, with all pictures being presented at three different picture sizes (120x120 pixels, $360 \times 360$ pixels, $600 \times 600$ pixels), the "picture size" dimension. In total, 288 trials were presented, session duration was 30 minutes. Each participant was instructed to rate all pictures according 
to their initial reaction on a digital Visual Analogue Scale anchored with 'not fearful at all' and 'extremely fearful'. The display was generated, and ratings were recorded using the Presentation software package (Version 16, Neurobehavioral Systems Inc., Albany, CA, USA).

\section{Analysis stimulus rating}

The rating data was analyzed in SPSS Statistics (IBM SPSS Statistics 21; IBM Corporation, Armonk, NY, USA). All subjective anxiety ratings from the natural spider picture set were analyzed using a repeated measures general linear model (GLM) with a linear contrast for the within subject factor picture size, and group as a between subject factor. All ratings from the parametric spider picture set were analyzed using a repeated measures GLM with linear contrasts for the within subject factors zoom, context, and picture size, and group as a between subject factor. Effect sizes were estimated using partial eta squared (Cohen, 1973). To investigate consistency across participants, the within subject analyses were repeated on single subject level.

\section{Procedure fMRI study}

Prior to scanning participants were instructed that blocks of spider pictures would be presented to them, and that they would have to rate their anxiety level on a 5 point Likert scale from $0=$ 'not fearful at all' to $4=$ 'extremely fearful' using a button box after each block. They were asked to refrain from any additional movement, and attend to all the pictures carefully. To accustom participants to the scanner environment, each 1-hour imaging session started with an anatomical imaging run, during which participants were shown silent cartoons to reduce anticipatory anxiety. During the following four experimental runs, blocks of spider pictures selected to provoke five different levels of anxiety (neutral, anxiety level 1, anxiety level 2, anxiety level 3, anxiety level 4) were presented. During each $6 \mathrm{~s}$ block, four pictures of the same category were presented for $1.5 \mathrm{~s}$ each. After each block a three second rating period, and a jittered resting period of 10.5-13.5 s followed. Six repetitions of the five conditions were presented in random order during each 11-minute functional run. Presentation software package was used to generate the visual display, and record all ratings.

The images were acquired at Maastricht Brain Imaging Centre (Maastricht University) on a 3T scanner, (Magnetom Allegra, Siemens Healthcare, Germany), equipped with a standard quadrature birdcage head coil. The participants were placed 
comfortably in the scanner and their heads were fixed with foam cushions to minimize motion. Functional images were acquired with a repeated single-shot echo-planar imaging (EPI) sequence that was optimized for imaging of subcortical and prefrontal areas by using an echo time to TE $=25 \mathrm{~ms}$ (Domsch et al., 2013; Morawetz et al., 2008; Weiskopf, Hutton, Josephs, Turner, \& Deichmann, 2007), slice thickness $3 \mathrm{~mm}$ with 10\% gap (Robinson, Windischberger, Rauscher, \& Moser, 2004; Weiskopf et al., 2007), and a tilted slice angle of $25-30^{\circ}$ (Deichmann, Gottfried, Hutton, \& Turner, 2003). Repetition time was $T R=1500 \mathrm{~ms}$, adjusted flip angle $F A=71^{\circ}$, field of view FOV $=224 \times 224 \mathrm{~mm}^{2}$, matrix size $=64 \times 64$, in-plane resolution $=3.5 \times 3.5 \mathrm{~mm}$, and bandwidth $=1736 \mathrm{~Hz} / \mathrm{Px}$. Per volume 25 slices were scanned, ensuring full coverage of the prefrontal cortex, subcortical structures and occipital cortex, while lacking full coverage of the parietal cortex. During all functional runs, heart rate was recorded using the scanner's standard MRI-compatible pulse oximeter, to monitor for increasing heart rate, the expected autonomous response in spider phobia (Prigatano \& Johnson, 1974; Sarlo, Palomba, Angrilli, \& Stegagno, 2002). Anatomical images were collected with a sequence based on the Alzheimer's Disease Neuroimaging Initiative (ADNI): $\mathrm{TR}=2250 \mathrm{~ms}, \mathrm{TE}=2.6 \mathrm{~ms}, \mathrm{FA}=9^{\circ}, \mathrm{FOV}=256 \times 256 \mathrm{~mm}^{2}, 192$ slices, voxel size $1 \times 1 \times 1$ $\mathrm{mm}^{3}$, with duration $8: 26$ minutes.

\section{Analysis fMRI study}

The subjective anxiety ratings were analyzed in SPSS Statistics using a repeated measures GLM with a linear contrast for the within subject factor anxiety level, and group as a between subject factor. Effect sizes were estimated using partial eta squared (Cohen, 1973). The pulse rate per condition was computed using a custom made MATLAB tool (R2010a; The MATHWORKS Inc., Natick, MA, USA), and submitted to the same analysis.

Functional and anatomical images were pre-processed and analyzed with BrainVoyager QX (Version 2.3, Brain Innovation B.V., Maastricht, The Netherlands), discarding the first four volumes of each functional run due to T1 saturation effects. The functional data was pre-processed using inter-scan slice-time correction, 3D rigidbody motion correction, temporal high-pass filtering with a GLM Fourier basis set, and spatial smoothing with an isotropic Gaussian kernel of 6-mm FWHM, and resampled to $3 \times 3 \times 3 \mathrm{~mm}^{3}$ voxels during brain normalization. None of the participants moved more than $1.9 \mathrm{~mm} /$ degrees in any direction. All anatomical and functional data were spatially 
normalized to Talairach space to enable whole-brain group analyses (Talairach \& Tournoux, 1988). All subsequent statistical group analyses were performed using a two-level random-effects GLM approach. The expected BOLD signal change was modeled by convolving the task predictors with a standard two-gamma hemodynamic response function. In all GLM analyses noise artifacts were modeled by including three translational, and three rotational head motion parameters (Hutton et al., 2011; Weissenbacher et al., 2009), a localized estimate of the white matter signal to model scanner artifacts (Jo, Saad, Simmons, Milbury, \& Cox, 2010), and the ventricular signal to estimate physiological artifacts (Birn, Murphy, Handwerker, \& Bandettini, 2009) in the GLM model. This combination of nuisance regressors has been recommended to be efficient in increasing the specificity (Hutton et al., 2011; Weissenbacher et al., 2009), as well as the reliability of the results (Guo et al., 2012).

For the whole-brain GLM analyses two different statistical models were used. In a first step, we adopted a categorical approach, where each of the five task conditions (neutral, anxiety level 1, anxiety level 2, anxiety level 3, anxiety level 4) was modeled by unweighted task regressors. This approach allowed us to contrast the mean of the four unweighted anxiety conditions with the neutral condition (balanced contrast values: $-4,1,1,1,1)$, computing the contrast generally used in fMRI anxiety provocation studies. In a second step, we adopted a parametric approach, in which the neutral condition was of no interest, and the four anxiety conditions were weighted to model a linear increase depending on the provoked anxiety level (balanced contrast values: $-3,-1,1,3)$. To detect only parametrically modulated regions which were taskactivated, the conjunction of this parametric contrast with a main contrast (contrast values: $1,1,1,1)$ was computed. Finally, all statistical maps were statistically thresholded using an initial voxel-threshold for statistical significance of $\alpha=0.05$ (Forman et al., 1995), and correcting for multiple comparisons using cluster-size thresholding with a cluster-level false positive rate of $\alpha=0.05$ (Forman et al., 1995; Goebel, Esposito, \& Formisano, 2006).

As a second, independent analysis, a correlational region-of-interest analysis was performed to investigate how well single-trial BOLD responses reflected the individual subjective anxiety ratings during anxiety provocation (anxiety level 1, anxiety level 2, anxiety level 3, anxiety level 4). Four a priori regions-of-interest (ROls) from the anxiety network, thalamus, amygdala, insula, and anterior cingulate (Del Casale et al., 2012; Etkin \& Wager, 2007), as well as dorsolateral prefrontal cortex, were selected based 
on the reviewed literature. All ROls were functionally defined based on thresholded maps computed from the unweighted group GLM, contrasting all conditions against baseline (contrast values $1,1,1,1$ ) across both groups, so that the ROI definition would not be biased by any group or condition differences. All significant voxels within a 15 $\mathrm{mm}$ range of the anatomical target coordinates were selected. From these ROls and two additional control regions (white matter, CSF) single-trial beta estimates were extracted voxelwise and averaged for each ROI using custom-made code in MATLAB. These ROI single-trial beta estimates were then correlated with the single trial subjective anxiety ratings on individual level. After applying Fisher $Z$ transformation to improve the normality of the calculated correlation coefficients (Fisher, 1928), we tested for significance on group level using t-tests in SPSS Statistics, with Bonferroni corrected thresholds for number of ROIs.

\section{Results}

\section{Stimulus rating}

As hypothesized, the evaluation of the spider picture sets showed that subjective anxiety ratings increased with greater experienced proximity (figure 3 ). The group difference in general subjective anxiety level was highly significant, being $43 \%$ for the natural spider picture set and $47 \%$ for the parametric spider picture set (natural spider picture set: $\mathrm{F}(1,38)=56.4, \mathrm{p}<0.001, \eta_{\mathrm{p}}^{2}=0.62$ parametric spider picture set: $\left.F(1,38)=122.4, p<0.001, \eta_{p}^{2}=0.78\right)$. As expected, there was an increase of anxiety with increasing picture size that was significantly stronger in the high-fear group than in the low-fear group for the picture size dimension (figure $\mathbf{3} \mathbf{a}, \mathbf{b}$ ). This additional increase was small but consistent across both picture sets (natural spider picture set: $F(1,38)=5.5, p<0.05, \eta_{p}^{2}=0.14 ;$ parametric spider picture set: $F(1,38)=4.5, p<$ $0.05, \eta_{p}^{2}=0.12$ ). Post hoc separate group analysis confirmed a linear increase of anxiety in both groups, with a larger increase in the high-fear group (low-fear group: natural spider picture set: $\mathrm{F}(1,19)=18.9, \mathrm{p}<0.001, \mathrm{n}_{\mathrm{p}}^{2}=0.53$; parametric spider picture set: $F(1,19)=20.9, p<0.001, \eta_{p}^{2}=0.55$; high-fear group: natural spider picture set: $F(1,19)=38.1, p<0.001, \eta_{p}^{2}=0.69$; parametric spider picture set: $F(1,19)=39.1$, $p<0.001, \eta_{p}^{2}=0.70$ ). This effect was highly consistent, being significant in 20/20 highfear and 16/20 low-fear participants on single subject level. Second, we observed a 

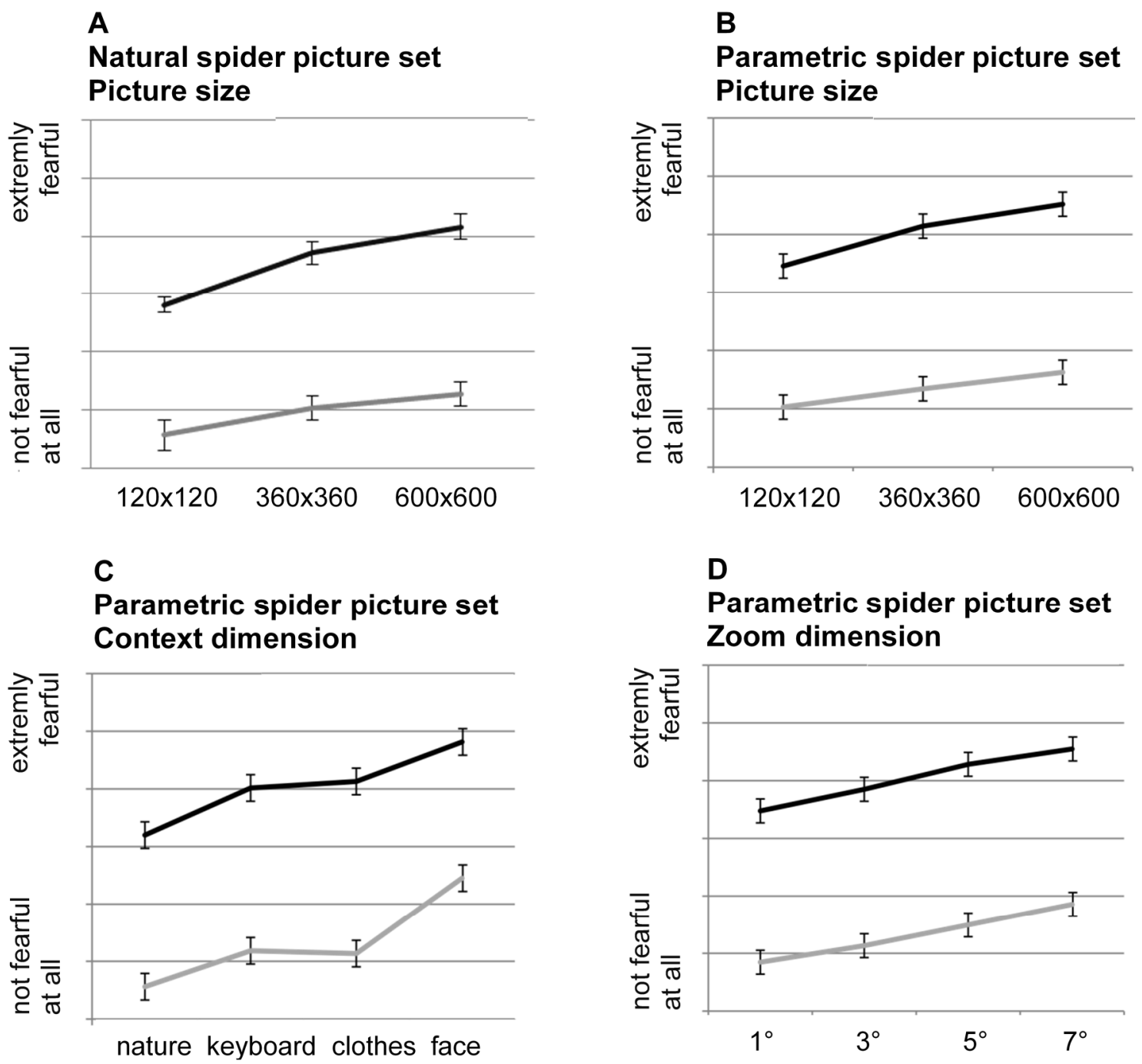

High-fear group

Low-fear group

\section{Figure 3. Results stimulus rating.}

The group averages of subjectively perceived anxiety from the behavioral rating study are depicted. Participants rated their anxiety on a visual analogue scale anchored by 'not fearful at all' and 'extremely fearful'. As hypothesized, subjective anxiety increased significantly with greater experienced proximity along all dimensions. This increase was significantly stronger in the high-fear group than in the low-fear group for the dimension picture size (A, B), and equally strong in both groups along the dimensions context and zoom (C, D). There was a large main difference in general anxiety level between groups. 
A

Natural spider picture set (120x120 pixel)

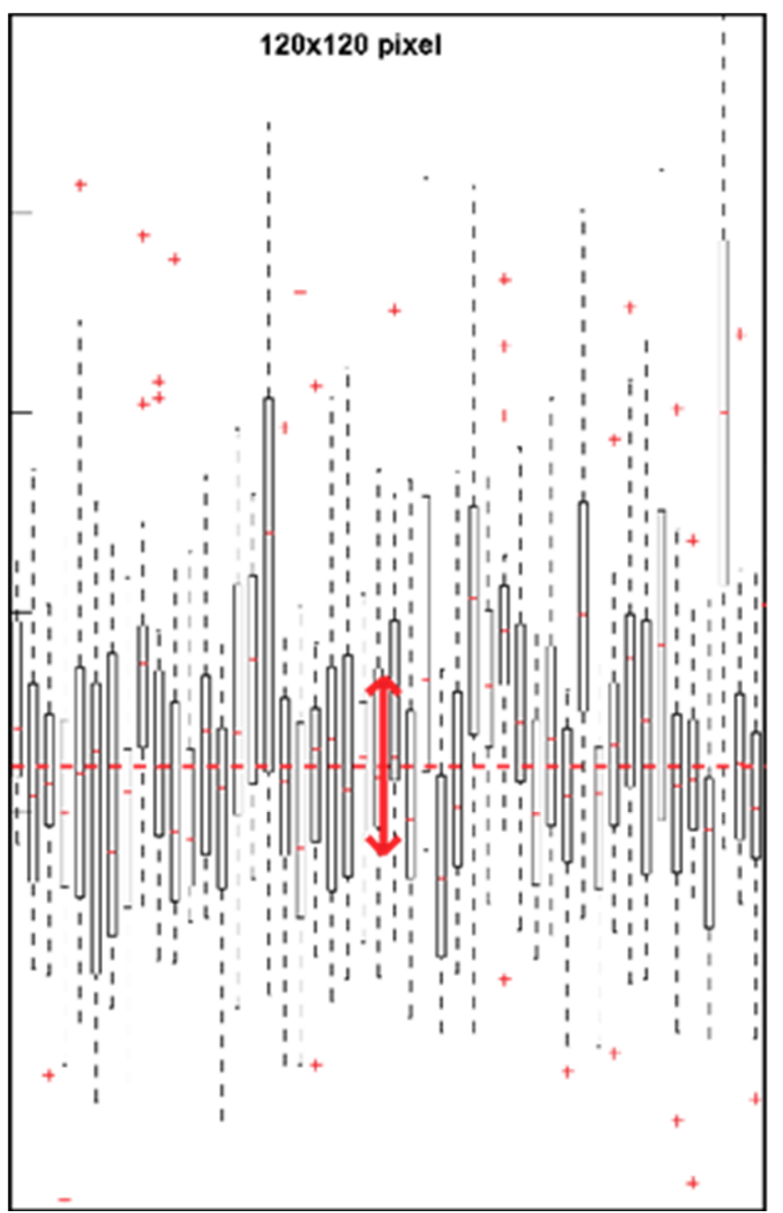

B

Parametric spider picture set

(120x120 pixel: sorted by zoom and context)

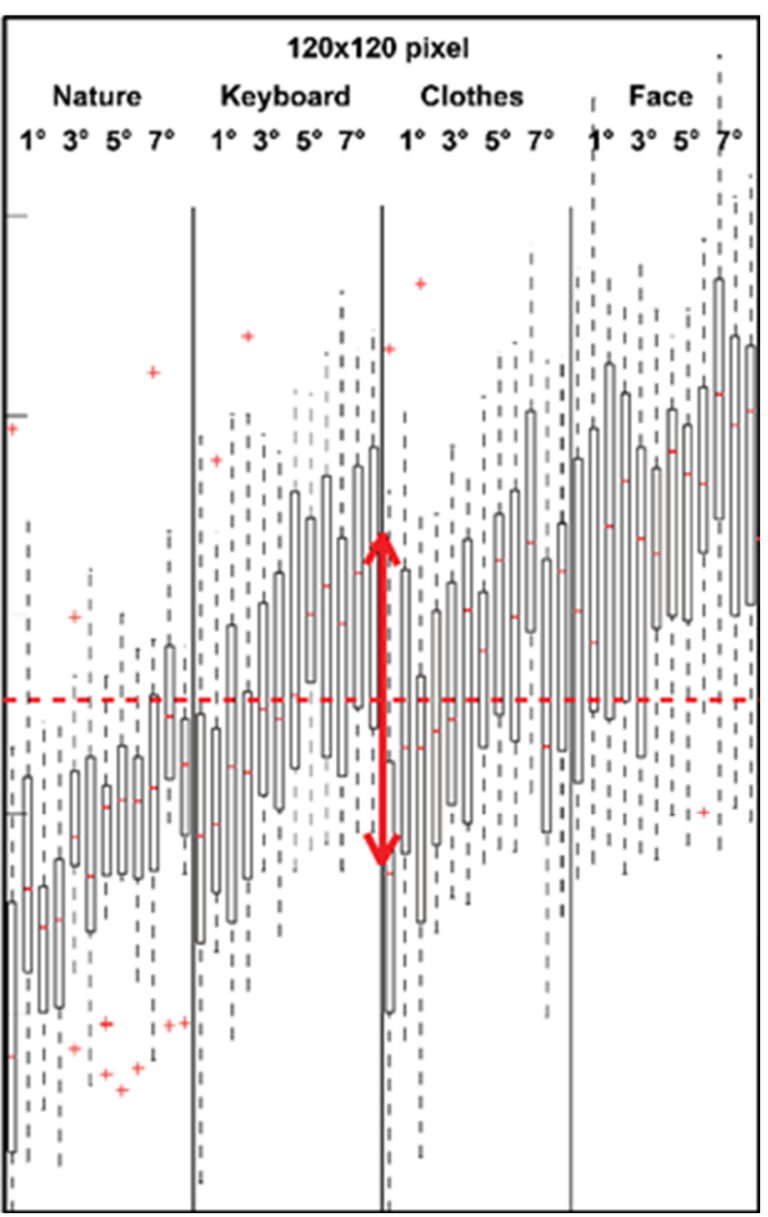

Figure 4. Raw rating data per picture.

The data from the behavioral rating study is depicted showing one boxplot per spider photograph (red crosses = outliers), demonstrating how high-fear participants rated the natural spider picture set (A), and the parametric spider picture set (B), presented at $120 \times 120$ pixel. While the stimuli of the natural spider picture set cannot be ordered a priori, the stimuli from the parametric spider picture set are sorted according to the experimental design (1. picture size, 2. context, and 3. zoom). The subjective ratings showed considerable variance across pictures for the parametric spider picture set, covering a large range from low to high anxiety (median $=$ dashed read line, standard deviation $=$ red arrow, B). This variance was considerably larger (+ $50 \%$ ) for the parametric spider picture set, in comparison to the natural spider picture set (A, B). Importantly, variance in the parametric spider picture set was structured according to the design, as hypothesized (B). 
gradual increase of anxiety along the dimensions context and zoom as hypothesized (figure $\mathbf{3} \mathbf{c}, \mathbf{d}$ ). The provoked anxiety level was modulated linearly along the dimension zoom, and three different anxiety levels were observable for the context dimension (1. nature, 2. keyboard/clothes, 3. face). Unexpectedly, the low and high-fear group showed an equally strong linear increase (context dimension: $F(1,38)=64.9, p<0.001$, $\eta_{p}^{2}=0.66$; zoom dimension: $\left.F(1,38)=93.3, p<0.001, \eta_{p}^{2}=0.73\right)$, with effect sizes for both dimensions being comparable to the picture size dimension. As for the picture size dimension, these effects were highly consistent across participants, being significant in 19/20 participants in both groups for both dimensions. Overall, the experimental manipulation showed increased anxiety with increasing proximity on single-subject level for all three dimensions.

An additional comparison of the two picture sets considering the variance across single pictures, rather than averaging, demonstrated that the range of the ratings acquired with the parametric spider picture set was larger than for the natural spider picture set (figure 4). The variance was $50 \%$ larger in the parametric spider picture set (parametric spider picture set: 120x120 pixel: SD = 13\%, 360×360 pixel: SD $=12$ $\%, 600 \times 600$ pixel: SD $=11 \%$, average: $12 \%$; natural spider picture set: $120 \times 120$ pixel: $\mathrm{SD}=7 \%, 360 \times 360$ pixel: $\mathrm{SD}=9 \%, 600 \times 600$ pixel: SD $=8 \%$, average: $8 \%$, figure 4 , variance depicted by the red arrows). Ratings from the parametric spider picture set therefore covered a larger range from low to high anxiety. Importantly, this variance was structured according to the experimental manipulation, allowing for experimental control (figure 4). The parametric spider picture set was used in the subsequent fMRI study, grouping the sixteen stimulus subcategories according to provoked anxiety (anxiety level 1, anxiety level 2, anxiety level 3, anxiety level 4), for a more simple experimental design (figure 5 ).

\section{fMRI study}

During scanning the second group of participants rated the final set of parametric stimuli (figure 5), which was designed to provoke four different levels of anxiety, with an added neutral category. The anxiety ratings showed a group difference of $47 \%$ between low and high-fear participants (figure 6; $F(1,12)=96.1, p<0.001, \eta_{p}^{2}=0.89$; missed trials high-fear group: $0.4 \%$, low-fear group: $1.6 \%$ ). The increase in anxiety was stronger in the high-fear group than in the low-fear group $(F(1,12)=30.8, p<0.001$, $\left.\eta_{p}{ }^{2}=0.72\right)$, with a large effect size for this interaction effect. Post hoc separate group 


\section{A Ratings from high-fear group}

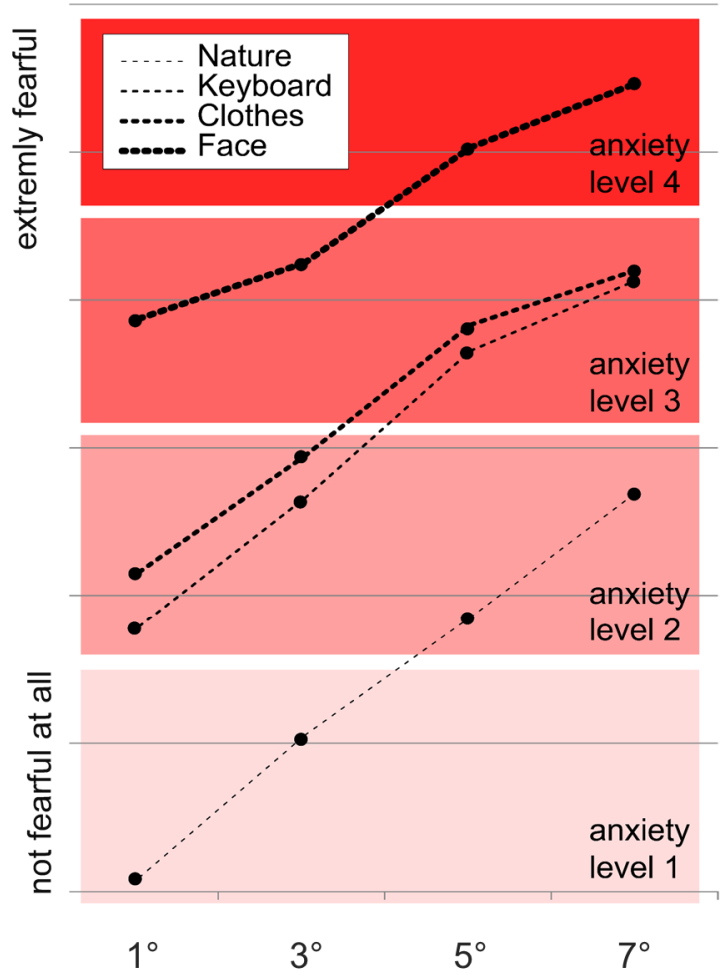

\section{B Grouping of stimuli subcategories}
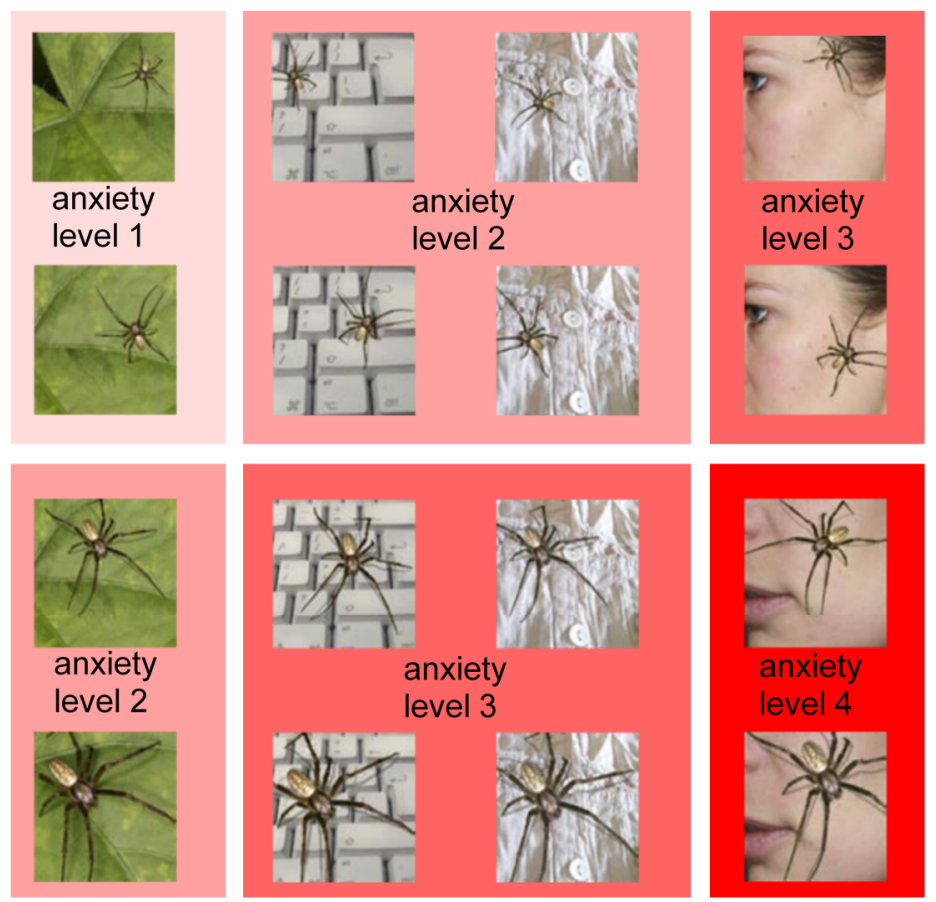

\section{Figure 5. Provoking four levels of anxiety.}

The subjective anxiety ratings from the high-fear participants of the behavioral rating study are depicted $(\mathbf{A})$. By dividing the covered range into four levels $(\mathbf{A})$, the sixteen stimulus subcategories of the parametric spider picture set were grouped into four different stimulus categories according to provoked anxiety level ( , anxiety level 2, anxiety level

3, anxiety level 4), as depicted by the background colors (B).

analysis showed a strong, consistent linear increase of subjective anxiety in the highfear group $\left(F(1,6)=266.3, p<0.001, \eta_{p}^{2}=0.98\right)$, and only a trend towards significance in the low-fear group $\left(F(1,6)=3.9, p=0.09, \eta_{p}^{2}=0.39\right)$. The analysis of the pulse rate data demonstrated that pulse was not directly influenced by subjective anxiety level. There was neither a significant group difference in pulse rate (high-fear group: 67 beats/minute, low-fear group: 69 beats/minute), nor a significant modulation by the level of anxiety (figure 7).

The whole-brain fMRI data analysis revealed the expected network. In the categorical analysis brain regions that were increasingly activated during spider versus neutral stimuli encompassed visual input areas, such as the superior colliculi, thalamus 

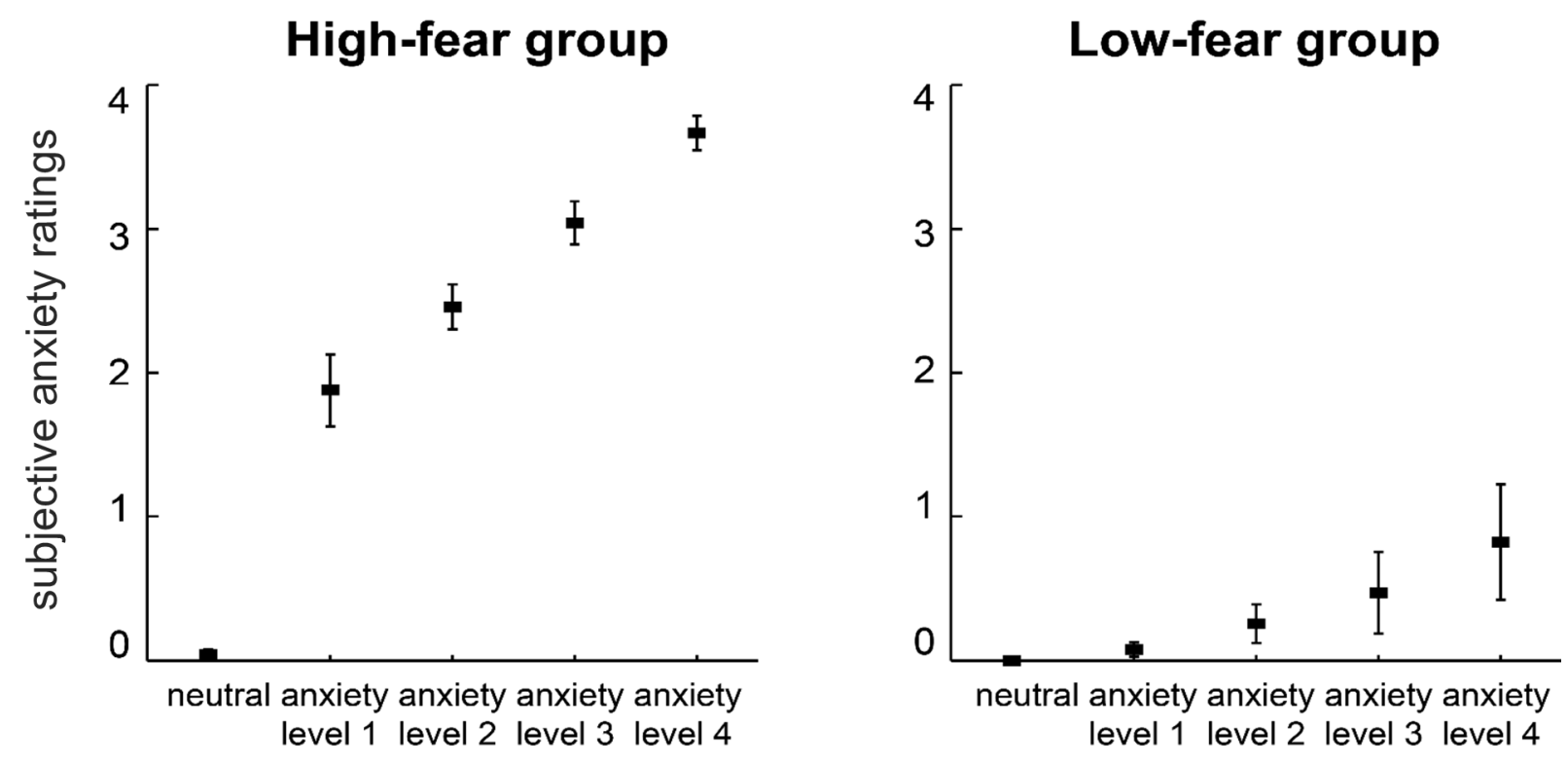

\section{Figure 6. Subjective anxiety during scanning.}

The subjective ratings from participants during scanning are presented. As hypothesized, the ratings show a linear modulation of anxiety level. This increase was highly significant in highfear participants, and only marginally significant in low-fear participants.

and visual cortex, medial-temporal regions hypothesized to be part of the anxiety network, as the dorsal anterior cingulate, anterior and mid insula, and, frontal regions involved in as the dorsal anterior cingulate, anterior and mid insula, and, frontal regions involved in higher cognitive tasks expected to be related to secondary cognitive processes, as the orbitofrontal cortex, and dorsolateral prefrontal cortex (table 3). Further activated regions included the dorsal striatum, ventrolateral prefrontal cortex, and posterior cingulate (table 3). It should be noted that the parietal cortex was not imaged due to reduced coverage. Generally, the regions early in the processing stream, such as visual input areas, were activated in both groups, with a stronger activation in the high-fear group, while medial-temporal and frontal regions only showed a significant response in the high-fear group (table 3). The parametric wholebrain analysis mapped the same core anxiety network as in the categorical analysis, with the amygdala being the only additionally detected area (table 4). Regions early in the processing stream, superior colliculi, thalamus and visual cortex, showed a significant linear increase of the BOLD response with increasing anxiety level for both groups, with a stronger increase in the high-fear group (table 4, figure 8a). Amygdala, 

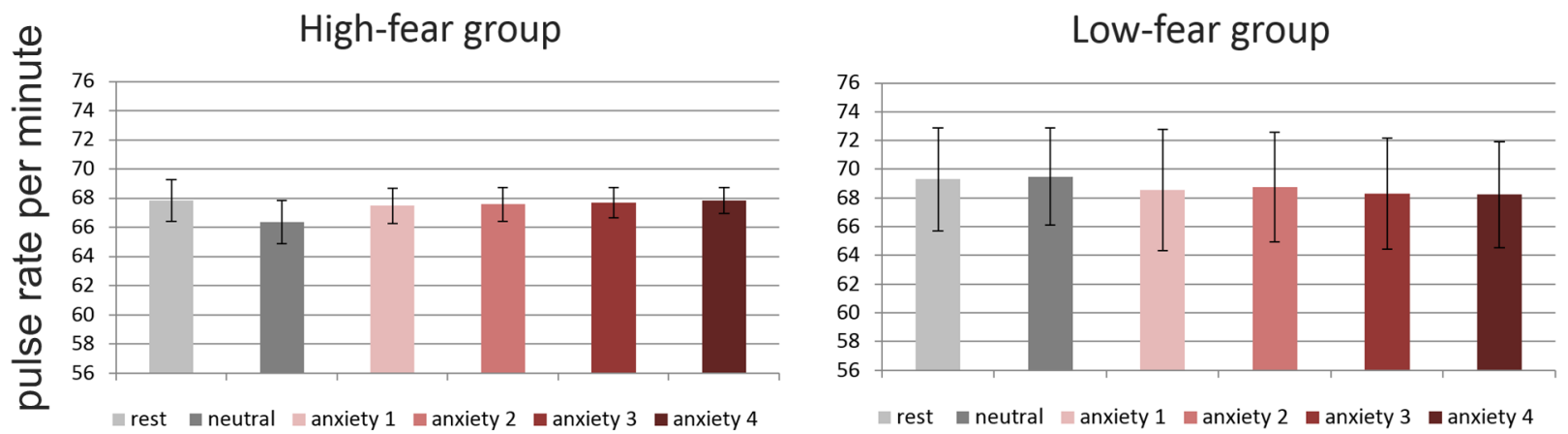

Figure 7. Pulse rate during scanning.

The pulse rate of the participants during scanning is shown. The pulse was neither modulated by anxiety level, nor was there a significant group difference.

striatum, and anterior insula, were significantly linearly modulated in the high-fear group, and showed mixed results for the low-fear group, as neither the analysis testing for a group difference (linear increase in high fear group only), nor the within-group analysis (linear increase in low fear group) were significant (table 4, figure 8a). However, the lack of a significant parametric effect in the low-fear group in the amygdala and anterior insula was due to a non-significant main effect, not a nonsignificant linear contrast. Overall, the data thus demonstrated a robust parametric modulation in the high and a weak parametric modulation in the low-fear group in amygdala and anterior insula (figure 8a). Finally, only three brain regions, the mid insula, the dorsal anterior cingulate cortex, and a left-lateralized ventrolateral prefrontal region, showed a linear modulation by provoked anxiety only in the high-fear group, and not in the low-fear group, with a significant interaction (linear increase in high fear group only), as in the subjective rating data (table 4, figure $\mathbf{8 b}$ ). Frontal regions, as orbitofrontal cortex and the dorsolateral prefrontal cortex, and the posterior cingulate did not show any parametric modulation of the BOLD response (table 4 , figure $8 c$ ).

The region-of-interest analysis, which investigated the correlation between singletrial estimated beta weights and single-trial subjective anxiety ratings, showed significant medium-sized correlations for the left and right amygdala (left: $t(6)=5.7, p$ $<0.001$, right: $\mathrm{t}(6)=4.5, \mathrm{p}<0.01)$, and the right mid insula $(\mathrm{t}(6)=4.0, \mathrm{p}<0.01)$ for the high-fear group only (figure 9). The correlations from other regions of the hypothesized anxiety network, thalamus, anterior insula, and dorsal anterior cingulate, were lower 
and not significant (figure 9). In regions outside of the anxiety network, the dorsolateral prefrontal cortex, white-matter, and cerebrospinal fluid, no significant correlations were observed.

\section{Table 3. Regions Activated by a Contrast of All Anxiety Levels (Irrespective of Level) versus Neutral Pictures}

\begin{tabular}{|c|c|c|c|c|c|}
\hline Brain Region & & $\begin{array}{l}\text { Brodman's } \\
\text { Areas (BA) }\end{array}$ & $\begin{array}{l}\text { High fear group: } \\
\text { anxiety > neutral }\end{array}$ & $\begin{array}{l}\text { High fear group } \\
>\text { low fear group } \\
\text { (anxiety > neutral) }\end{array}$ & $\begin{array}{l}\text { Low fear group: } \\
\text { anxiety > neutral }\end{array}$ \\
\hline & & & $x / y / z$ (no of voxels) & $x / y / z$ (no of voxels) & $\mathrm{x} / \mathrm{y} / \mathrm{z}$ (no of voxels) \\
\hline \multicolumn{6}{|c|}{ Midbrain } \\
\hline \multirow{2}{*}{$\begin{array}{c}\text { Brainstem \& } \\
\text { Superior Colliculi }\end{array}$} & $\mathrm{L}$ & & $-8 /-27 /-3$ & $-7 /-19 /-9$ & $-12 /-23 /-3 \quad(14)$ \\
\hline & $\mathrm{R}$ & & $8 /-25 /-3$ & $8 /-20 /-9$ & $14 /-26 /-3$ \\
\hline \multicolumn{6}{|c|}{ Visual areas } \\
\hline \multirow[t]{2}{*}{ Visual cortex } & $\mathrm{L}$ & $17,18,19,37$ & $-45 /-71 /-7 \quad(804)$ & $-21 /-87 /-5 \quad(312)$ & $-52 /-58 / 7$ \\
\hline & $\mathrm{R}$ & $17,18,19,37$ & $30 /-75 /-12 \quad(678)$ & $15 /-77 /-5 \quad(271)$ & $51 /-63 / 4$ \\
\hline \multicolumn{6}{|c|}{ Subcortical areas } \\
\hline \multirow[t]{2}{*}{ Thalamus } & $\mathrm{L}$ & & $-10 /-22 / 15 \quad(152)$ & $-9 /-7 / 12$ & --- \\
\hline & $\mathrm{R}$ & & $6 /-11 / 7$ & $3 /-12 / 13 \quad(42)$ & --- \\
\hline \multirow[t]{2}{*}{ Striatum } & $\mathrm{L}$ & & $-7 / 10 / 10$ & $-9 / 10 / 12 \quad(61)$ & --- \\
\hline & $\mathrm{R}$ & & $8 / 6 / 9$ & $13 / 13 / 15$ & --- \\
\hline \multirow[t]{2}{*}{ Amygdala } & $\mathrm{L}$ & & --- & --- & --- \\
\hline & $\mathrm{R}$ & & --- & --- & --- \\
\hline \multicolumn{6}{|c|}{ Medial-temporal areas } \\
\hline \multirow[t]{2}{*}{ Anterior Insula } & $\mathrm{L}$ & 13 & $-34 / 16 / 6$ & --- & --- \\
\hline & $\mathrm{R}$ & 13 & $39 / 22 / 10$ & --- & --- \\
\hline \multirow[t]{2}{*}{ Mid Insula } & $\mathrm{L}$ & 13 & $-34 / 8 / 6$ & --- & --- \\
\hline & $\mathrm{R}$ & 13 & $38 / 7 / 0$ & ---- & --- \\
\hline \multirow{2}{*}{$\begin{array}{l}\text { Anterior } \\
\text { Cingulate }\end{array}$} & $\mathrm{L}$ & 24,32 & $-9 / 25 / 31$ & --- & --- \\
\hline & $\mathrm{R}$ & 24,32 & $5 / 25 / 32$ & --- & --- \\
\hline \multicolumn{6}{|c|}{ Frontal areas } \\
\hline \multirow{2}{*}{$\begin{array}{c}\text { Ventrolateral } \\
\text { prefrontal cortex }\end{array}$} & $\mathrm{L}$ & $44,45,47$ & $-50 / 14 / 1$ & --- & --- \\
\hline & $\mathrm{R}$ & $44,45,47$ & $51 / 22 / 7$ & ---- & --- \\
\hline \multirow{2}{*}{$\begin{array}{l}\text { Orbitofrontal } \\
\text { cortex }\end{array}$} & $\mathrm{L}$ & 10,11 & $-22 / 43 /-3$ & ---- & --- \\
\hline & $\mathrm{R}$ & 10,11 & $36 / 59 / 12$ & $32 / 51 / 12$ & --- \\
\hline \multirow{2}{*}{$\begin{array}{c}\text { Dorsolateral } \\
\text { prefrontal cortex }\end{array}$} & $\mathrm{L}$ & $8,9,46$ & $-45 / 22 / 28$ & $-50 / 14 / 36$ & --- \\
\hline & $\mathrm{R}$ & $8,9,46$ & $40 / 22 / 31$ & $40 / 29 / 31$ & --- \\
\hline \multicolumn{6}{|c|}{ Posterior areas } \\
\hline \multirow[t]{2}{*}{ Posterior Cingulate } & $\mathrm{L}$ & 23,29 & $-7 /-35 / 22 \quad(200)$ & --- & --- \\
\hline & $\mathrm{R}$ & 23,29 & $3 /-38 / 21 \quad(123)$ & --- & --- \\
\hline $\begin{array}{l}L=\text { Left Hemisphere } \\
\text { Talairach coordinate } \\
\text { voxels }\left(3 \times 3 \times 3 \mathrm{~mm}^{3}\right)\end{array}$ & $\begin{array}{l}R \\
\text { S } 0\end{array}$ & $\begin{array}{l}\text { Right Hemisphe } \\
\text { e most significa } \\
\text { ported. }\end{array}$ & loxel and the nu & of significantly a & functional \\
\hline
\end{tabular}

Results from the whole-brain random-effects GLM categorical analysis ( $p<0.05$ corrected at cluster level). The table summarizes which brain regions showed higher activation during anxiety compared to neutral pictures in high fear participants (Left: "High fear group: anxiety > neutral"), and low fear participants (Right: "Low fear group: anxiety > neutral"), and which regions were significantly more activated in high fear than low fear participants (middle: "High fear group > low fear group anxiety (anxiety > neutral)"). 


\section{Table 4. Regions Activated by Parametric Anxiety Level Contrast}

\begin{tabular}{|c|c|c|c|c|c|c|}
\hline $\begin{array}{l}\text { Brain } \\
\text { Region }\end{array}$ & & $\begin{array}{l}\text { Brodman's } \\
\text { Areas (BA) }\end{array}$ & $\begin{array}{l}\text { High fear group: } \\
\text { linear increase }\end{array}$ & $\begin{array}{l}\text { High fear group } \\
>\text { low fear } \\
\text { group } \\
\text { (linear increase) }\end{array}$ & $\begin{array}{c}\text { Interaction: } \\
\text { linear increase } \\
\text { high fear group } \\
\text { only }\end{array}$ & $\begin{array}{l}\text { Low fear group: } \\
\text { linear increase }\end{array}$ \\
\hline & & & $x / y / z$ (no voxels) & $\mathrm{x} / \mathrm{y} / \mathrm{z}$ (no voxels) & $x / y / z$ (no voxels) & $\mathrm{x} / \mathrm{y} / \mathrm{z}$ (no voxels) \\
\hline \multicolumn{7}{|c|}{ Midbrain } \\
\hline \multirow{2}{*}{$\begin{array}{c}\text { Brainstem \& } \\
\text { Superior } \\
\text { Colliculi }\end{array}$} & $\mathrm{L}$ & & $-7 /-19 /-11 \quad(71)$ & $-4 /-28 /-3$ & --- & $-2 /-26 /-2 \quad(44)$ \\
\hline & $\mathrm{R}$ & & $6 /-22 /-9 \quad(63)$ & $5 /-22 /-6$ & --- & $3 /-25 /-3 \quad(66)$ \\
\hline \multicolumn{7}{|c|}{ Visual areas } \\
\hline \multirow[t]{2}{*}{ Visual cortex } & $\mathrm{L}$ & $18,19,37$ & $-22 /-67 /-12(349)$ & $-25 /-68 /-11(709)$ & --- & $-37 /-76 /-6(593)$ \\
\hline & $\mathrm{R}$ & $18,19,37$ & $35 /-65 /-12(322)$ & $44 /-65 /-12(604)$ & --- & $29 /-76 / 2 \quad(456)$ \\
\hline \multicolumn{7}{|c|}{ Subcortical areas } \\
\hline \multirow{2}{*}{ Thalamus } & $\mathrm{L}$ & & $-4 /-17 / 6$ & $-10 /-14 / 15$ & --- & $-12 / 28 / 1$ \\
\hline & $\mathrm{R}$ & & $8 /-20 / 9 \quad(118)$ & $8 /-13 / 15 \quad(155)$ & --- & $16 /-29 / 3 \quad(123)$ \\
\hline \multirow[t]{2}{*}{ Striatum } & $\mathrm{L}$ & & $-21 / 8 / 9$ & $-21 / 10 / 6 \quad(158)$ & --- & --- \\
\hline & $\mathrm{R}$ & & $20 / 3 / 9$ & $20 / 4 / 8$ & --- & --- \\
\hline \multirow[t]{2}{*}{ Amygdala } & $\mathrm{L}$ & & $-19 /-5 /-10 \quad(21)$ & $-19 /-5 /-10 \quad(27)$ & --- & --- \\
\hline & $\mathrm{R}$ & & $25 /-2 /-12 \quad(17)$ & $28 /-2 /-12 \quad(20)$ & --- & --- \\
\hline \multicolumn{7}{|c|}{ Medial-temporal areas } \\
\hline \multirow{2}{*}{$\begin{array}{l}\text { Anterior } \\
\text { Insula }\end{array}$} & $\mathrm{L}$ & 13 & $-36 / 16 / 11 \quad(119)$ & $-34 / 14 / 11(176)$ & --- & --- \\
\hline & $\mathrm{R}$ & 13 & $35 / 11 / 15 \quad(101)$ & $32 / 10 / 13 \quad(159)$ & --- & --- \\
\hline \multirow[t]{2}{*}{ Mid Insula } & $\mathrm{L}$ & 13 & $-37 /-2 / 10 \quad(105)$ & $-38 /-2 / 10 \quad(146)$ & $-37 / 4 / 9$ & --- \\
\hline & $\mathrm{R}$ & 13 & $37 / 2 / 6$ & $38 /-2 / 7 \quad(137)$ & $37 / 4 / 8$ & $\begin{array}{l}-- \\
\end{array}$ \\
\hline \multirow{2}{*}{$\begin{array}{l}\text { Anterior } \\
\text { Cingulate }\end{array}$} & $\mathrm{L}$ & 24,32 & $-8 / 16 / 37 \quad(114)$ & $-8 / 17 / 39 \quad(134)$ & $-7 / 16 / 28 \quad(54)$ & --- \\
\hline & $\mathrm{R}$ & 24,32 & $9 / 7 / 36 \quad(77)$ & $5 / 7 / 43 \quad(108)$ & $5 / 7 / 36$ & --- \\
\hline \multicolumn{7}{|c|}{ Frontal areas } \\
\hline \multirow{2}{*}{$\begin{array}{l}\text { Ventrolateral } \\
\text { prefrontal }\end{array}$} & $\mathrm{L}$ & $44,45,47$ & $-50 / 13 / 4$ & $-50 / 15 / 4$ & $-52 / 8 / 6$ & $\begin{array}{l}--- \\
\end{array}$ \\
\hline & $\mathrm{R}$ & $44,45,47$ & $51 / 21 / 3$ & $51 / 22 / 7$ & --- & --- \\
\hline \multirow{2}{*}{$\begin{array}{l}\text { Orbitofrontal } \\
\text { cortex }\end{array}$} & $\mathrm{L}$ & & --- & --- & --- & --- \\
\hline & $\mathrm{R}$ & & --- & --- & --- & --- \\
\hline \multirow{2}{*}{$\begin{array}{l}\text { Dorsolateral } \\
\text { prefrontal }\end{array}$} & $\mathrm{L}$ & & --- & --- & --- & --- \\
\hline & $\mathrm{R}$ & & --- & --- & --- & --- \\
\hline \multicolumn{7}{|c|}{ Posterior areas } \\
\hline \multirow{2}{*}{$\begin{array}{l}\text { Posterior } \\
\text { Cingulate }\end{array}$} & $\mathrm{L}$ & & --- & --- & --- & --- \\
\hline & $\mathrm{R}$ & & --- & --- & --- & --- \\
\hline
\end{tabular}

Results from the whole-brain random-effects GLM parametric analysis ( $p<0.05$ corrected at cluster level). The table summarizes which brain regions demonstrated a linear increase of activation with increasing anxiety in high fear participants (Left: "High fear group: linear increase"), and low fear participants (Right: "Low fear group: linear increase"). Further, which regions showed a stronger linear increase the high fear group compared to the low fear group (middle left: "High fear group > low fear group anxiety (linear increase)"), and finally, which regions demonstrated a statistically significant interaction effect for a parametric increase in the high fear group, but not in the low fear group (Middle right: "Interaction: linear increase in high fear group only"). 
Figure 8. Modulation of BOLD response according to anxiety levels.

Voxels showing a linear increase of BOLD response with increasing anxiety level in high-fear participants (whole-brain random-effects parametric GLM analysis, $p<0.05$ corrected at cluster level) are mapped on an averaged brain (yellow-orange, A, B, C). For the prefrontal cortex the anxiety versus neutral contrast is depicted (random-effects categorical GLM analysis, $p<0.05$ corrected at cluster level) (pink-red, C). Regions-of-interest (white borders) were defined based on the conjunction of all voxels significantly activated in both analysis. From these regions average beta weights of the estimated BOLD response were extracted for each condition (neutral, anxiety level 1, anxiety level 2, anxiety level 3, anxiety level 4) from high-fear (middle panel), and low-fear participants (right panel). The results illustrate that subcortical regions demonstrated a linear modulation of BOLD response according to provoked anxiety in both groups, with a stronger increase in the high-fear group (A). The BOLD response in the mid insula and dorsal anterior cingulate most closely resembled subjective anxiety levels, showing a linear modulation for the high-fear group only (B). Frontal regions were not linearly modulated by anxiety level, but showed equally increased activation for all levels of anxiety (C). 


\section{A Subcortical areas (thalamus and amygdala)}
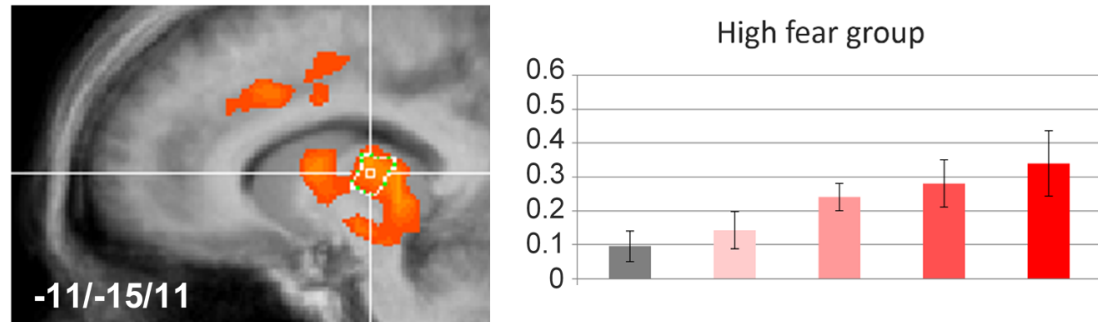

Low fear group
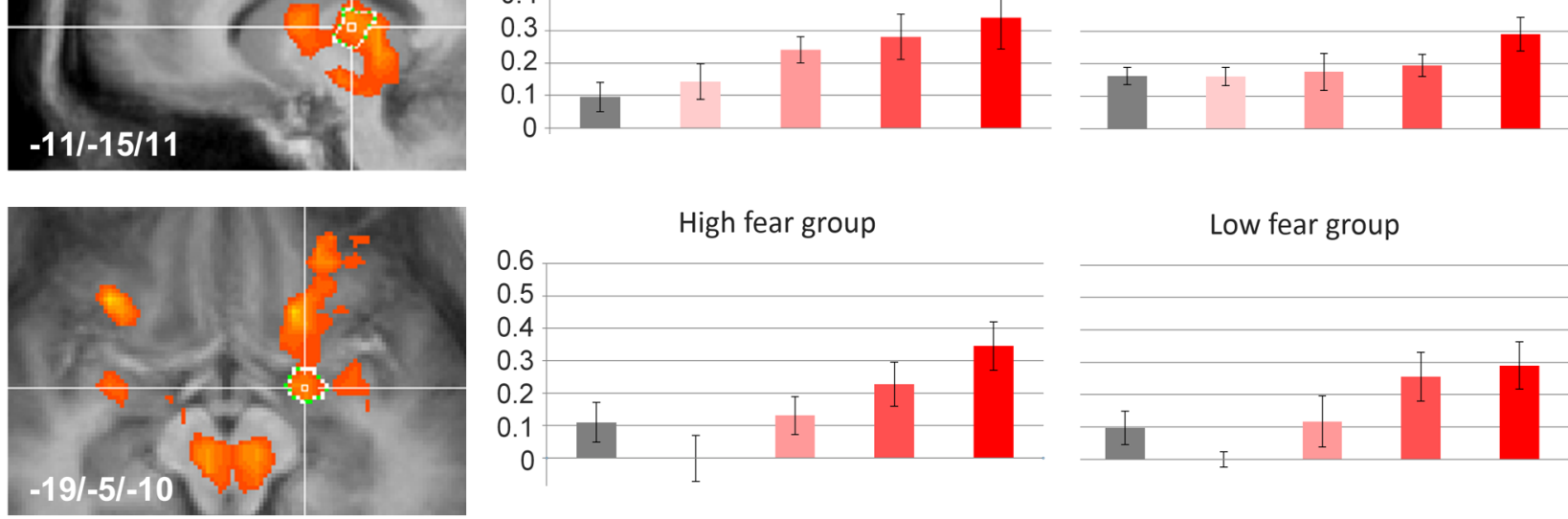

B Medial-temporal areas (mid insula and dorsal anterior cingulate)
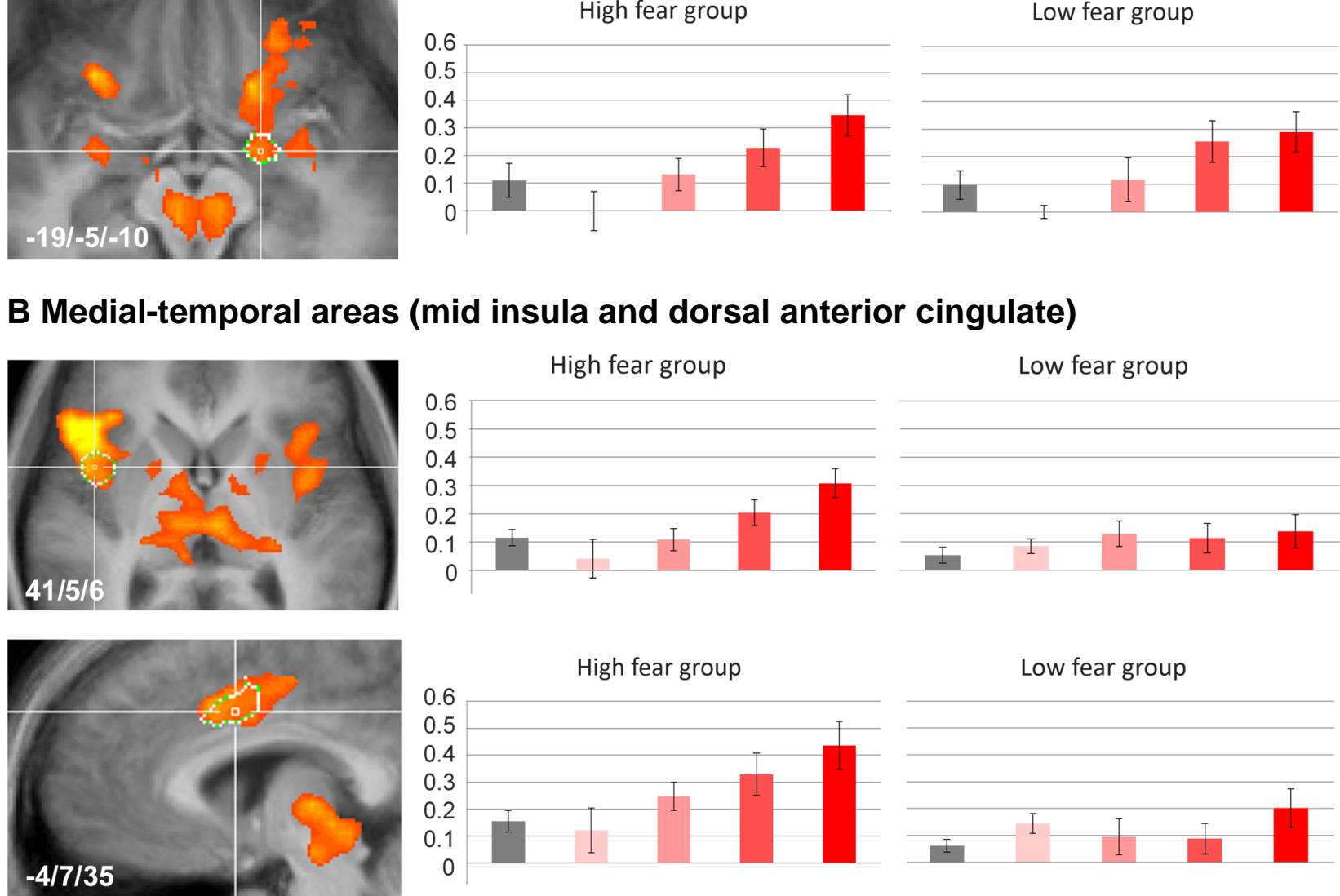

\section{Frontal areas (dorsolateral prefrontal cortex)}
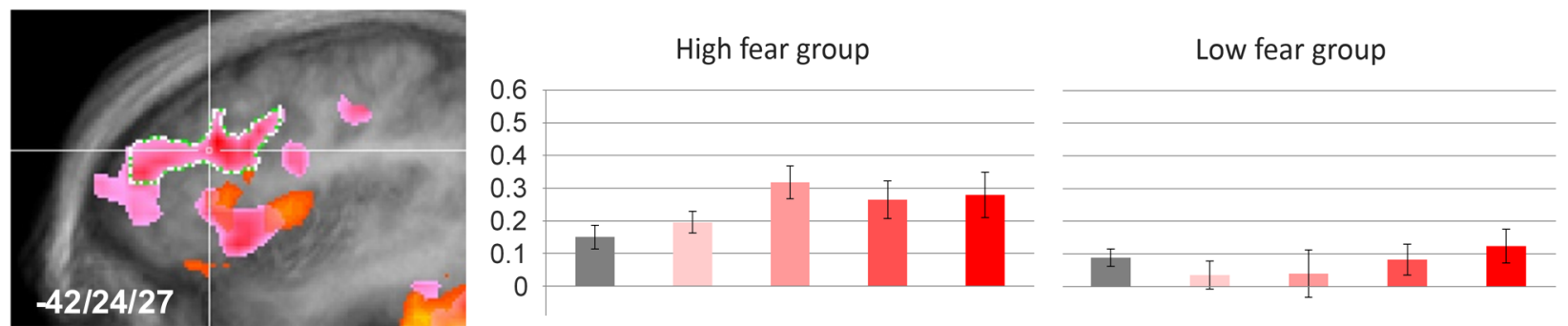

neutral anxiety1 anxiety2 anxiety3 $\square$ anxiety4 


\section{Correlation beta weights with subjective ratings}

\section{High-fear group}

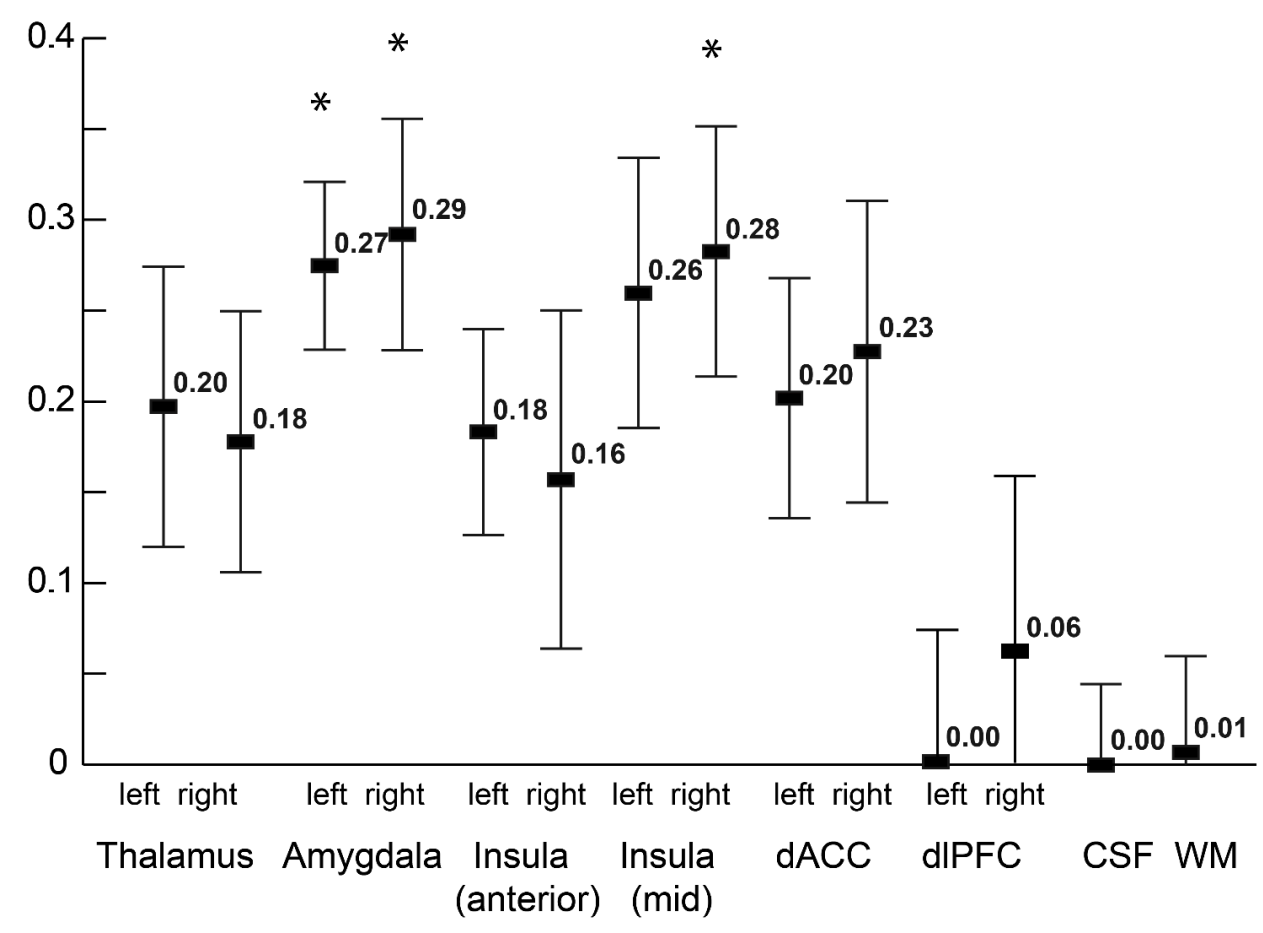

\section{Low-fear group}

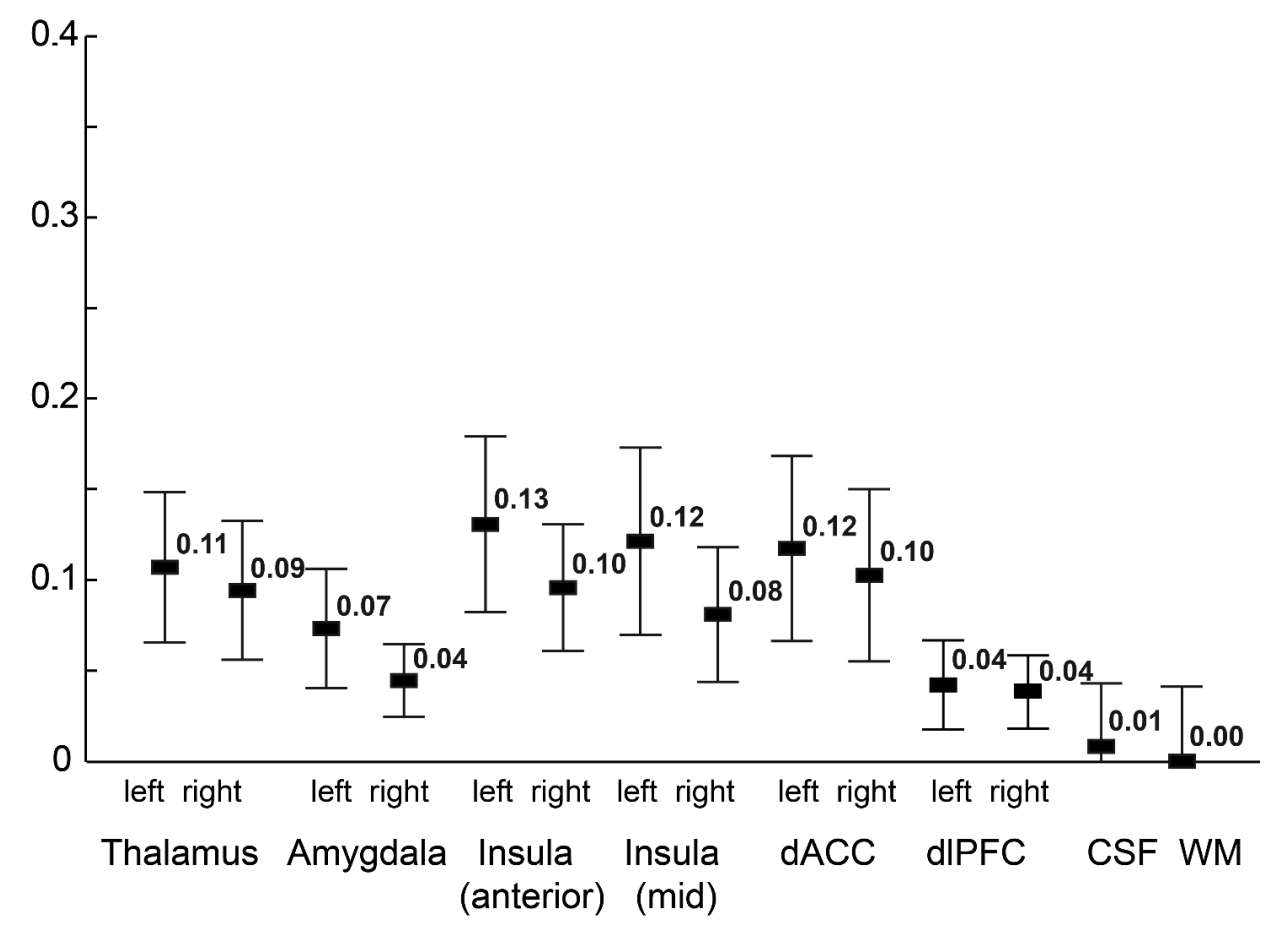




\section{Figure 9. Correlation of beta weights with subjective anxiety ratings.}

The correlations between single-trial estimated beta weights and single-trial subjective anxiety ratings are plotted for the high-fear group (A) and low-fear group (B), significance is indicated by an asterisk (Bonferroni corrected). We found significant medium-sized correlations in the amygdala and right mid insula of the high-fear participants only $(\mathbf{A})$. Other regions from the anxiety network showed lower correlations ( $\mathrm{dACC}=$ dorsal anterior cingulate), while the BOLD signal in the dorsolateral prefrontal cortex (dIPFC), and control regions (CSF $=$ cerebrospinal fluid, $\mathrm{WM}=$ white matter) was not correlated with subjective anxiety level, as expected.

\section{Discussion}

The results from the stimulus rating demonstrated that the pictures from the "parametric spider picture set" were as effective as photographs from the "natural spider picture set". Both picture sets were equally well suited to provoke different levels of anxiety depending on individual fear of spider, and presented picture size. However, with the "parametric spider picture set" a greater range of different anxiety levels could be provoked in a controlled way, as predicted by the stimulus manipulation. In general all applied stimulus manipulations had the desired effect, subjective anxiety increased with greater experienced proximity along all dimensions as hypothesized. For all dimensions the tested linear increase exhibited large effect sizes, and results were very consistent across participants. Depending on the manipulated stimulus aspect, there was a stronger increase in the high-fear group than the low-fear group. However, this additional effect was relatively small. High and low-fear participants therefore seemed to estimate threat in a similar way, independent of their individual fear level, with high-fear participants being only slightly more vulnerable to increasing proximity.

In the less comfortable scanner environment the difference between high and lowfear participants was much more pronounced than during the behavioral stimulus rating study. While the difference between both groups in mean anxiety level remained similar, the provoked anxiety increase with increasing proximity was much larger in the high-fear group. Brain regions representing subjective anxiety quantitatively would therefore be expected to show a clear interaction effect of the BOLD response, with a linear increase in the high-fear group, but not in the low-fear group. In order to ensure that BOLD effects would not be a mere reflection of physiological effects, we analyzed 
heart rate, the physiological parameter most often found to be correlated with an increase of anxiety in spider phobia (Prigatano \& Johnson, 1974; Sarlo et al., 2002). The analysis of the acquired pulse data showed that heart rate did not increase dependent on subjective anxiety, thus not being a confounding factor for the fMRI data analysis. As a precautionary step, we additionally controlled for other residual physiological artifacts by integrating CSF and white matter signals as confounds in the design matrix of the fMRI GLM analyses (Murphy, Birn, Handwerker, Jones, \& Bandettini, 2009).

Based on the fMRI GLM analyses we were able to map the expected networks, including the brain regions most often activated during anxiety symptom provocation, the amygdala, insula, anterior cingulate, and thalamus (Del Casale et al., 2012; Etkin \& Wager, 2007), and visual, medial and prefrontal areas. In general, there was a clear processing hierarchy, with the parametric modulation of the signal becoming less pronounced at higher processing levels. At the lower level, the input level encompassing subcortical and visual structures, brain regions such as the midbrain areas in the brain stem, superior colliculi, thalamus, and visual cortex, showed a linear increase of BOLD response with increasing anxiety in both groups, as was hypothesized for regions involved in threat monitoring. The amygdala, and the anterior insula, showed a linear modulation in the high-fear group, with a weak linear effect in the low-fear group, also indicating a functional role of these regions in threat monitoring. Overall, threat monitoring thus included two aspects: the visual analysis of the potentially threatening stimulus in visual input areas, and the actual risk estimation, being computed in the amygdala, and anterior insula. The amygdala was the core region involved in threat monitoring, as the correlation with the subjective anxiety ratings on an individual level was highest. These results corroborate previous research in healthy participants supporting a key role of the amygdala in initial threat monitoring (Mobbs et al., 2010). The results are also in line with research showing that the amygdala is involved in rapid, automatic, initial processing of phobia-related stimuli during initial detection of fear-relevant stimuli, which is largely independent of attention and awareness (Carlsson et al., 2004; Straube, Mentzel, \& Miltner, 2006), and consistent with a model suggesting that the anterior insula evaluates how external stimuli will influence the body state (Paulus \& Stein, 2006).

At a higher processing level, three regions, the mid insula, the dorsal anterior cingulate, and the left ventrolateral prefrontal cortex, showed a clear linear increase in 
the high-fear but not the low-fear group. These results suggest that these regions hold a representation of subjectively perceived anxiety levels. Importantly, these regions have not been found to be directly involved in threat monitoring (Mobbs et al., 2010). The insula has generally been implicated in the representation of bodily states and conscious arousal (Critchley, Mathias, \& Dolan, 2001). Recently, a posterior-to-anterior gradient from a representation of bodily states in the posterior insula, to subjective evaluations in the anterior insula has been suggested (Craig, 2011; Grupe \& Nitschke, 2013). Subjective threat evaluations would then be integrated with information on bodily states in the mid-insula (Craig, 2011; Grupe \& Nitschke, 2013), which is the brain region shown to be hyperactive in phobic patients, but not in healthy participants during fear conditioning (Etkin \& Wager, 2007). The mid insula may thus have a role in representing subjective anxiety levels by integrating bodily states and threat relevant information. As the BOLD signal in the mid insula was highly correlated with subjective anxiety ratings, it seems to hold a primary representation of subjective anxiety levels, while the dorsal anterior cingulate and ventrolateral prefrontal cortex may hold a secondary representation. It has been previously suggested that bodily states are remapped in the cingulate cortex in order to integrate them with sensory, motor and attentional inputs (Critchley et al., 2001), with the main functional role of the anterior cingulate being the allocation of control and attention (Bush, Luu, \& Posner, 2000; Carter, Botvinick, \& Cohen, 1999; Paus, 2001; Shenhav, Botvinick, \& Cohen, 2013). The ventrolateral prefrontal cortex has been implicated in effortful control of emotion (Ochsner et al., 2004; Phan et al., 2005). A representation of subjective anxiety levels in these brain regions would therefore be the basis for allocation of resources depending on experienced threat.

At the highest processing level, prefrontal regions as the orbitofrontal and dorsolateral prefrontal cortex, were activated during anxiety, but did not show a linear increase dependent on anxiety levels in any of the two groups, as hypothesized for regions involved in secondary cognitive processes. A role of frontal brain regions in supporting secondary cognitive processes evoked by increased anxiety is supported by the literature. The orbitofrontal cortex has been described as a brain region involved in encoding the memory of affective value (Gottfried \& Dolan, 2004; Milad \& Rauch, 2007; Milad et al., 2005; Rolls \& Grabenhorst, 2008), while the dorsolateral prefrontal cortex has been implicated in representation of context information and goal setting (Courtney, Ungerleider, Keil, \& Haxby, 1997; D’Esposito et al., 1995; Fuster, 1990; 
Grupe \& Nitschke, 2013; MacDonald, Cohen, Stenger, \& Carter, 2000). A limitation of the presented study is that due to limited coverage of the imaging sequence, not the whole network of regions involved in secondary cognitive processes could be described, mainly parietal regions involved in maladaptive control were not imaged (Grupe \& Nitschke, 2013).

Overall, we found a clear processing hierarchy with at least three different levels of processing by employing a parametric fMRI anxiety provocation design in spider phobic participants. We demonstrated that at a lower processing level, in visual and subcortical structures including the amygdala, the BOLD response was linearly modulated according to perceived proximity in both groups, as hypothesized for brain regions involved in threat monitoring. At an intermediate level, in the mid insula and dorsal anterior cingulate, we found a linear relationship between BOLD response and perceived proximity in the high-fear group only, as expected for regions involved in the representation of subjective anxiety. Importantly, the whole brain analysis therefore indicated different functional roles for the amygdala and mid insula, which have both been previously implicated as being the core regions of the anxiety network (Etkin \& Wager, 2007). The analysis further confirmed, that the BOLD response in activated higher cognitive prefrontal regions did not show a quantitative relation to perceived proximity in any of the two groups, as hypothesized for regions supporting secondary cognitive processes.

\section{Conclusions}

We conclude that anxiety processing is organized hierarchically, and that a parametric fMRI design can be used to tease apart different processing levels. This study furthermore confirms that brain regions involved in threat monitoring and representation of subjective anxiety show a linear relationship between BOLD response and anxiety level. These regions therefore indeed hold a quantitative representation of subjective anxiety levels. Research aiming at evaluating and developing new therapeutic approaches for anxiety disorders, may therefore focus on monitoring changes in these regions employing a parametric symptom provocation paradigm. 


\section{Acknowledgements}

We want to thank Ed Nieuwenhuys for providing the excellent photographs of European spiders, Valentin Kemper for his generous support with optimizing fMRI data acquisition and preprocessing, Joel Reithler for providing tools for the physiological data analysis, and Armin Heinecke, Judith Eck, and Jan Zimmermann for their very helpful suggestions regarding the analysis. The authors gratefully acknowledge the support of the BrainGain Smart Mix Program of The Netherlands Ministry of Economic Affairs and The Netherlands Ministry of Education, Culture and Science (grant number: SSM06011). 


\section{References}

American Psychiatric Association. (2000). Diagnostic and Statistical Manual of Mental Disorders, Fourth Edition, Text Revision (DSM-IV-TR). Text (Vol. 1). Washington, DC: American Psychiatric Association. doi:10.1176/appi.books.9780890423349

Barlow, D. H. (2002). True Alarms, False Alarms, and Learned (Conditioned) Anxiety. In D. H. Barlow (Ed.), Anxiety and its disorders: the nature and treatment of anxiety and panic (2nd ed., pp. 219-251). New York: The Guilford Press.

Birn, R. M., Murphy, K., Handwerker, D. A., \& Bandettini, P. A. (2009). fMRI in the presence of task-correlated breathing variations. Neurolmage, 47(3), 1092-104. doi:10.1016/j.neuroimage.2009.05.030

Bush, G., Luu, P., \& Posner, M. (2000). Cognitive and emotional influences in anterior cingulate cortex. Trends in Cognitive Sciences, 4(6), 215-222.

Carlsson, K., Petersson, K. M., Lundqvist, D., Karlsson, A., Ingvar, M., \& Ohman, A. (2004). Fear and the amygdala: manipulation of awareness generates differential cerebral responses to phobic and fear-relevant (but nonfeared) stimuli. Emotion, 4(4), 340-353. doi:10.1037/1528-3542.4.4.340

Carter, C. S., Botvinick, M. M., \& Cohen, J. D. (1999). The contribution of the anterior cingulate cortex to executive processes in cognition. Reviews in the Neurosciences, 10(1), 49-57.

Cohen, J. (1973). Eta-Squared and Partial Eta-Squared in Fixed Factor Anova Designs. Educational and Psychological Measurement, 33(1), 107-112. doi:10.1177/001316447303300111

Courtney, S. M., Ungerleider, L. G., Keil, K., \& Haxby, J. V. (1997). Transient and sustained activity in a distributed neural system for human working memory. Nature, 386, 608-611.

Craig, A. (Bud). (2011). Significance of the insula for the evolution of human awareness of feelings from the body. Annals of the New York Academy of Sciences, 1225, 72-82. doi:10.1111/j.1749-6632.2011.05990.x

Critchley, H. D., Mathias, C. J., \& Dolan, R. J. (2001). Neuroanatomical basis for firstand second-order representations of bodily states. Nature Neuroscience, 4(2), 207-12. doi:10.1038/84048

D’Esposito, M., Detre, J. A., Alsop, D. C., Shin, R. K., Atlas, S., \& Grossman, M. (1995). The neural basis of the central executive system of working memory. Nature, 378(6554), 279-281.

Deichmann, R., Gottfried, J. ., Hutton, C., \& Turner, R. (2003). Optimized EPI for fMRI studies of the orbitofrontal cortex. Neurolmage, 19(2), 430-441. doi:10.1016/S1053-8119(03)00073-9 
Del Casale, A., Ferracuti, S., Rapinesi, C., Serata, D., Piccirilli, M., Savoja, V., ... Girardi, P. (2012). Functional neuroimaging in specific phobia. Psychiatry Research, 202(3), 181-97. doi:10.1016/j.pscychresns.2011.10.009

Dilger, S., Straube, T., Mentzel, H.-J., Fitzek, C., Reichenbach, J. R., Hecht, H., ... Miltner, W. H. R. (2003). Brain activation to phobia-related pictures in spider phobic humans: an event-related functional magnetic resonance imaging study. Neuroscience Letters, 348(1), 29-32. doi:10.1016/S0304-3940(03)00647-5

Domsch, S., Linke, J., Heiler, P. M., Kroll, A., Flor, H., Wessa, M., \& Schad, L. R. (2013). Increased BOLD sensitivity in the orbitofrontal cortex using slicedependent echo times at 3 T. Magnetic Resonance Imaging, 31(2), 201-11. doi:10.1016/j.mri.2012.06.020

Etkin, A., \& Wager, T. D. (2007). Functional neuroimaging of anxiety: a meta-analysis of emotional processing in PTSD, social anxiety disorder, and specific phobia. The American Journal of Psychiatry, 164(10), 1476-1488. doi:10.1176/appi.ajp.2007.07030504

Fisher, R. A. (1928). The General Sampling Distribution of the Multiple Correlation Coefficient. Proceedings of the Royal Society A: Mathematical, Physical and Engineering Sciences, 121(788), 654-673. doi:10.1098/rspa.1928.0224

Forman, S. D., Cohen, J. D., Fitzgerald, M., Eddy, W. F., Mintun, M. a, \& Noll, D. C. (1995). Improved assessment of significant activation in functional magnetic resonance imaging (fMRI): use of a cluster-size threshold. Magnetic Resonance in Medicine, 33(5), 636-47.

Fuster, J. M. (1990). Prefrontal cortex and the bridging of temporal gaps in the perception-action cycle. Annals of the New York Academy of Sciences, 608, 31829; discussion 330-6.

Goebel, R., Esposito, F., \& Formisano, E. (2006). Analysis of functional image analysis contest (FIAC) data with brainvoyager QX: From single-subject to cortically aligned group general linear model analysis and self-organizing group independent component analysis. Human Brain Mapping, 27(5), 392-401. doi:10.1002/hbm.20249

Goossens, L., Schruers, K., Peeters, R., Griez, E., \& Sunaert, S. (2007). Visual presentation of phobic stimuli: amygdala activation via an extrageniculostriate pathway? Psychiatry Research, 155(2), 113-20. doi:10.1016/j.pscychresns.2006.12.005

Gottfried, J. a, \& Dolan, R. J. (2004). Human orbitofrontal cortex mediates extinction learning while accessing conditioned representations of value. Nature Neuroscience, 7(10), 1144-52. doi:10.1038/nn1314

Grupe, D. W., \& Nitschke, J. B. (2013). Uncertainty and anticipation in anxiety: an integrated neurobiological and psychological perspective. Nature Reviews. Neuroscience, 14(7), 488-501. doi:10.1038/nrn3524 
Guo, C. C., Kurth, F., Zhou, J., Mayer, E. a, Eickhoff, S. B., Kramer, J. H., \& Seeley, W. W. (2012). One-year test-retest reliability of intrinsic connectivity network fMRI in older adults. Neurolmage, 61(4), 1471-83. doi:10.1016/j.neuroimage.2012.03.027

Heinzel, A., Bermpohl, F., Niese, R., Pfennig, A., Pascual-Leone, A., Schlaug, G., \& Northoff, G. (2005). How do we modulate our emotions? Parametric fMRI reveals cortical midline structures as regions specifically involved in the processing of emotional valences. Brain Research. Cognitive Brain Research, 25(1), 348-58. doi:10.1016/j.cogbrainres.2005.06.009

Helmchen, C., Mohr, C., Erdmann, C., Binkofski, F., \& Büchel, C. (2006). Neural activity related to self- versus externally generated painful stimuli reveals distinct differences in the lateral pain system in a parametric fMRI study. Human Brain Mapping, 27(9), 755-65. doi:10.1002/hbm.20217

Hutton, C., Josephs, O., Stadler, J., Featherstone, E., Reid, a, Speck, O., ... Weiskopf, N. (2011). The impact of physiological noise correction on fMRI at $7 \mathrm{~T}$. Neurolmage, 57(1), 101-12. doi:10.1016/j.neuroimage.2011.04.018

Jansma, J. M., Ramsey, N. F., Coppola, R., \& Kahn, R. S. (2000). Specific versus nonspecific brain activity in a parametric N-back task. Neurolmage, 12(6), 68897. doi:10.1006/nimg.2000.0645

Jo, H. J., Saad, Z. S., Simmons, W. K., Milbury, L. a, \& Cox, R. W. (2010). Mapping sources of correlation in resting state FMRI, with artifact detection and removal. Neurolmage, 52(2), 571-82. doi:10.1016/j.neuroimage.2010.04.246

Kessler, R. C., Chiu, W. T., Demler, O., Merikangas, K. R., \& Walters, E. E. (2005). Prevalence, severity, and comorbidity of 12-month DSM-IV disorders in the National Comorbidity Survey Replication. Archives of General Psychiatry, 62(6), 617-627.

Klorman, R., Weerts, T. C., Hastings, J. E., Melamed, B. G., \& Lang, P. J. (1974). Psychometric description of some specific-fear questionnaires. Behavior Therapy, 5(3), 401-409.

Klumpp, H., Angstadt, M., Nathan, P. J., \& Phan, K. L. (2010). Amygdala reactivity to faces at varying intensities of threat in generalized social phobia: an event-related functional MRI study. Psychiatry Research, 183(2), 167-9. doi:10.1016/j.pscychresns.2010.05.001

Kwong, K. K., Belliveau, J. W., Chesler, D. a, Goldberg, I. E., Weisskoff, R. M., Poncelet, B. P., ... Turner, R. (1992). Dynamic magnetic resonance imaging of human brain activity during primary sensory stimulation. Proceedings of the National Academy of Sciences of the United States of America, 89(12), 5675-9.

MacDonald, A. W., Cohen, J. D., Stenger, V. A., \& Carter, C. S. (2000). Dissociating the role of the dorsolateral prefrontal and anterior cingulate cortex in cognitive control. Science (New York, N.Y.), 288(5472), 1835-1838. 
Milad, M. R., Quinn, B. T., Pitman, R. K., Orr, S. P., Fischl, B., \& Rauch, S. L. (2005). Thickness of ventromedial prefrontal cortex in humans is correlated with extinction memory. Proceedings of the National Academy of Sciences, 102(30), 10706-11. doi:10.1073/pnas.0502441102

Milad, M. R., \& Rauch, S. L. (2007). The role of the orbitofrontal cortex in anxiety disorders. Annals of the New York Academy of Sciences, 1121, 546-61. doi:10.1196/annals.1401.006

Mobbs, D., Yu, R., Rowe, J. B., Eich, H., Feldmanhall, O., \& Dalgleish, T. (2010). Neural activity associated with monitoring the oscillating threat value of a tarantula. Proceedings of the National Academy of Sciences, 107(47), 2058220586. doi:10.1073/pnas.1009076107

Mohr, C., Binkofski, F., Erdmann, C., Büchel, C., \& Helmchen, C. (2005). The anterior cingulate cortex contains distinct areas dissociating external from selfadministered painful stimulation: a parametric fMRI study. Pain, 114(3), 347-57. doi:10.1016/j.pain.2004.12.036

Morawetz, C., Holz, P., Lange, C., Baudewig, J., Weniger, G., Irle, E., \& Dechent, P. (2008). Improved functional mapping of the human amygdala using a standard functional magnetic resonance imaging sequence with simple modifications. Magnetic Resonance Imaging, 26(1), 45-53. doi:10.1016/j.mri.2007.04.014

Murphy, K., Birn, R. M., Handwerker, D. a, Jones, T. B., \& Bandettini, P. a. (2009). The impact of global signal regression on resting state correlations: are anti-correlated networks introduced? Neurolmage, 44(3), 893-905. doi:10.1016/j.neuroimage.2008.09.036

Ochsner, K. N., Ray, R. D., Cooper, J. C., Robertson, E. R., Chopra, S., Gabrieli, J. D. E., \& Gross, J. J. (2004). For better or for worse : neural systems supporting the cognitive down- and up-regulation of negative emotion. Neurolmage, 23, 483499. doi:10.1016/j.neuroimage.2004.06.030

Ost, L.-G. (2008). Cognitive behavior therapy for anxiety disorders: 40 years of progress. Nordic Journal of Psychiatry, 62(1), 5-10. doi:10.1080/08039480802315590

Paulus, M. P., \& Stein, M. B. (2006). An insular view of anxiety. Biological Psychiatry, 60(4), 383-7. doi:10.1016/j.biopsych.2006.03.042

Paus, T. (2001). Primate anterior cingulate cortex: where motor control, drive and cognition interface. Nature Reviews. Neuroscience, 2(6), 417-24. doi:10.1038/35077500

Phan, K. L., Fitzgerald, D. a, Nathan, P. J., Moore, G. J., Uhde, T. W., \& Tancer, M. E. (2005). Neural substrates for voluntary suppression of negative affect: a functional magnetic resonance imaging study. Biological Psychiatry, 57(3), 210-9. doi:10.1016/j.biopsych.2004.10.030 
Prigatano, G. P., \& Johnson, H. J. (1974). Autonomic nervous system changes associated with a spider phobic reaction. Journal of Abnormal Psychology, 83(2), 169-177. doi:10.1037/h0036476

Rainville, P. (1997). Pain Affect Encoded in Human Anterior Cingulate But Not Somatosensory Cortex. Science, 277(5328), 968-971. doi:10.1126/science.277.5328.968

Rao, S. M., Bandettini, P. A., Binder, J. R., Bobholz, J. A., Hammeke, T. A., Stein, E. A., \& Hyde, J. S. (1996). Relationship between finger movement rate and functional magnetic resonance signal change in human primary motor cortex. Journal of Cerebral Blood Flow and Metabolism, 16(6), 1250-1254. doi:10.1097/00004647-199611000-00020

Robinson, S., Windischberger, C., Rauscher, a, \& Moser, E. (2004). Optimized 3 T EPI of the amygdalae. Neurolmage, 22(1), 203-10. doi:10.1016/j.neuroimage.2003.12.048

Rolls, E. T., \& Grabenhorst, F. (2008). The orbitofrontal cortex and beyond: from affect to decision-making. Progress in Neurobiology, 86(3), 216-44. doi:10.1016/j.pneurobio.2008.09.001

Sarlo, M., Palomba, D., Angrilli, A., \& Stegagno, L. (2002). Blood phobia and spider phobia: Two specific phobias with different autonomic cardiac modulations. Biological Psychology, 60(2-3), 91-108.

Schienle, A., Schäfer, A., Walter, B., Stark, R., \& Vaitl, D. (2005). Brain activation of spider phobics towards disorder-relevant, generally disgust- and fear-inducing pictures. Neuroscience Letters, 388(1), 1-6. doi:10.1016/j.neulet.2005.06.025

Sheehan, D. V, Lecrubier, Y., Sheehan, K. H., Amorim, P., Janavs, J., Weiller, E., ... Dunbar, G. C. (1998). The Mini-International Neuropsychiatric Interview (M.I.N.I.): the development and validation of a structured diagnostic psychiatric interview for DSM-IV and ICD-10. The Journal of Cinical Psychiatry, 59 Suppl 2, 22-33;quiz 34-57. doi:10.1590/S1516-44462000000300003

Shenhav, A., Botvinick, M. M., \& Cohen, J. D. (2013). The expected value of control: an integrative theory of anterior cingulate cortex function. Neuron, 79(2), 217-40. doi:10.1016/j.neuron.2013.07.007

Straube, T., Glauer, M., Dilger, S., Mentzel, H.-J., \& Miltner, W. H. R. (2006). Effects of cognitive-behavioral therapy on brain activation in specific phobia. Neurolmage, 29(1), 125-35. doi:10.1016/j.neuroimage.2005.07.007

Straube, T., Mentzel, H.-J., \& Miltner, W. H. R. (2006). Neural mechanisms of automatic and direct processing of phobogenic stimuli in specific phobia. Biological Psychiatry, 59(2), 162-70. doi:10.1016/j.biopsych.2005.06.013

Straube, T., Schmidt, S., Weiss, T., Mentzel, H.-J., \& Miltner, W. H. R. (2009). Dynamic activation of the anterior cingulate cortex during anticipatory anxiety. Neurolmage, 44(3), 975-81. doi:10.1016/j.neuroimage.2008.10.022 
Szymanski, J. (1995). Fear of spiders questionnaire. Journal of Behavior Therapy and Experimental Psychiatry, 26(1), 31-34.

Talairach, J., \& Tournoux, P. (1988). Co-planar stereotaxic atlas of the human brain: 3-dimensional proportional system: an approach to cerebral imaging. Neuropsychologia (Vol. 39). New York: Thieme Medical Publishers.

Tamietto, M., \& de Gelder, B. (2010). Neural bases of the non-conscious perception of emotional signals. Nature Reviews. Neuroscience, 11(10), 697-709. doi:10.1038/nrn2889

Veltman, D. J., Tuinebreijer, W. E., Winkelman, D., Lammertsma, A. a, Witter, M. P., Dolan, R. J., \& Emmelkamp, P. M. G. (2004). Neurophysiological correlates of habituation during exposure in spider phobia. Psychiatry Research, 132(2), 14958. doi:10.1016/j.pscychresns.2004.09.001

Weiskopf, N., Hutton, C., Josephs, O., Turner, R., \& Deichmann, R. (2007). Optimized EPI for fMRI studies of the orbitofrontal cortex: compensation of susceptibilityinduced gradients in the readout direction. MAGMA, 20(1), 39-49. doi:10.1007/s10334-006-0067-6

Weissenbacher, A., Kasess, C., Gerstl, F., Lanzenberger, R., Moser, E., \& Windischberger, C. (2009). Correlations and anticorrelations in resting-state functional connectivity MRI: A quantitative comparison of preprocessing strategies. Neurolmage, 47(4), 1408-1416.

Wittchen, H.-U., \& Jacobi, F. (2005). Size and burden of mental disorders in Europe-a critical review and appraisal of 27 studies. European Neuropsychopharmacology: The Journal of the European College of Neuropsychopharmacology, 15(4), 357-76. doi:10.1016/j.euroneuro.2005.04.012 
110 | CHAPTER 3 


\section{4}

fMRI neurofeedback for regulating anxiety:

"fMRI neurofeedback enhances efficacy of cognitive reappraisal for anxiety regulation in spider phobia."

Based on: Zilverstand, A., Sorger, B., Sarkheil, P., \& Goebel, R. fMRI neurofeedback enhances efficacy of cognitive reappraisal for anxiety regulation in spider phobia. [in preparation]. 


\begin{abstract}
Anxiety disorders represent a maladaptive response following the perception of a real or imagined threat. Changing erroneous thoughts and behavior about anxiety inducing situations through cognitive reappraisal is regarded an effective strategy for anxiety regulation. Neurophysiological models have established the dorsolateral prefrontal cortex as a key structure of a regulatory network, which modulates the response of regions involved in expression of anxiety, including the insula. The presented study investigated if neurofeedback derived from functional magnetic resonance imaging (fMRI) signal of left dorsolateral prefrontal cortex and right insula, as an indicator of engagement and regulation success, respectively, would facilitate learning how to selfregulate anxiety. Eighteen female spider phobics participated in an exploratory, randomized, controlled trial with blinding of the participants, completing one training session of ninety minutes on using cognitive reappraisal strategies during four increasing levels of anxiety provocation. The experimental group received neurofeedback, while the control group completed the same procedure without receiving feedback information. Results from subjective rating of anxiety levels and the fMRI data analysis showed that neurofeedback participants achieved better downregulation of anxiety during the training than control participants. Both groups showed a decrease of anxiety three months after the training. We conclude that neurofeedback enhanced the efficacy of learning to regulate clinical levels of anxiety, making it a promising approach in treatment of patients with anxiety disorders. In a broader context this shows that hemodynamic signals can be used as an efficient tool in a therapeutic process.
\end{abstract}




\section{Introduction}

The promise of neurofeedback is that providing someone with relevant information on their individual brain processes may facilitate positive change. In fMRI neurofeedback, 'neural' information from circumscribed brain areas is 'fed back', enabling participants to modulate target brain processes, as for example regulatory processes in the presented study. The feasibility of deriving meaningful information from brain processes through functional magnetic resonance imaging (fMRI) has been shown in a series of studies performed in healthy participants (Goebel, Zilverstand, \& Sorger, 2010; Weiskopf, 2012). The reported studies demonstrate that it is possible to target brain regions specifically, and that providing individuals with this information results in related changes in behavior, emotion, and cognition (Goebel et al., 2010; Weiskopf, 2012). In a clinical context, neurofeedback training with fMRI signals has first been applied in chronic pain patients, who learned how to regulate activation levels in the rostral anterior cingulate cortex (DeCharms et al., 2005). Patients were able to reduce pain symptoms by gaining control through self-regulation of this brain region, involved in conscious pain perception (DeCharms et al., 2005). The reduction in pain was correlated with the achieved change in activation level (DeCharms et al., 2005). This was the first fMRI neurofeedback study demonstrating that an increase in control on a neural level can directly influence clinical symptoms.

A second pioneering clinical study demonstrated that tinnitus patients were able to voluntarily attenuate the response in hyper-activated auditory cortex, which lead to a mild reduction of tinnitus symptoms in a subgroup of patients (Haller, Birbaumer, \& Veit, 2010). This study showed that directly targeting hyper-activated regions may have beneficial effects. An exemplary trial with Parkinson's patients demonstrated that patients were also able to up-regulate chronically under-activated regions, in this case the supplementary motor cortex (Subramanian et al., 2011). The neurofeedback group, but not the control group, showed a sustained improvement of symptoms and motor skills during finger tapping (Subramanian et al., 2011). A similar approach was used in depressed patients, who learned to up-regulate brain regions responsive to positively valenced visual stimuli, and therefore improved their ability to generate positive emotions (Linden et al., 2012). The neurofeedback training had a positive effect on current mood, as well as on clinical symptoms, and improvement was correlated with achieved increases in activation level (Linden et al., 2012). Importantly, this study 
pursued an integrative approach, as the neurobiological feedback was used as a tool for shaping cognitive strategies. The findings were replicated in a second study with depressed patients, who learned to increase their positive emotions based on amygdala neurofeedback (Young et al., 2014). Again, fMRI neurofeedback was used as a tool for shaping cognitive strategies through up-regulation of activation in subcortical brain structures, which typically show an attenuated response during positive emotion processing in depressed patients (Young et al., 2014). A first study investigating neurofeedback training as means for learning anxiety regulation in a group with subclinical symptoms of contamination anxiety, pursued a similar approach. Prior to the training participants were instructed which therapeutic techniques may be useful for reducing contamination anxiety. The neurofeedback group was then able to refine these strategies through learning to modulate activation levels in the orbitofrontal cortex, an area implicated in contamination anxiety (Scheinost et al., 2013). The neurofeedback group, but not the control group achieved a lasting reduction of anxiety (Scheinost et al., 2013).

These exciting first clinical neurofeedback studies demonstrated that at least in some patient groups neural signals can be used as an efficient tool in a therapeutic process. At the same time, demand for further improving treatment of anxiety disorders is large, as success rates have been stagnating (Ost, 2008), while anxiety remains the most common mental health problem in most countries (Kessler et al., 2011), with the year-prevalence at $12-18 \%$ in Europe and the USA (Kessler, Petukhova, Sampson, Zaslavsky, \& Wittchen, 2012; Wittchen \& Jacobi, 2005). Research on incorporating cognitive techniques into standard exposure in-vivo treatment is therefore ongoing. Two sorts of general cognitive emotion regulation strategies are available, techniques that reduce the emotional impact of a situation by reinterpreting it, 'cognitive reappraisal', and techniques for suppressing emotions or distancing oneself (Amstadter, 2008; Gross, 2002). While it has been shown that the application of cognitive reappraisal strategies in anxiety treatment has a favorable long-term outcome, anxiety suppression and distancing have adverse effects in the long run (Amstadter, 2008; Farmer \& Kashdan, 2012; Kamphuis \& Telch, 2000; Sloan \& Telch, 2002). Further integration of cognitive reappraisal strategies into the treatment of anxiety disorders has therefore been recommended (Amstadter, 2008).

fMRI research on brain processes underlying cognitive reappraisal has mostly involved healthy participants, who learned to reappraise different negative emotions. 
Two brain networks were distinguished, the 'regulatory network' that encompasses prefrontal and cingulate control systems for supporting cognitive control, and the 'emotional network' consisting of limbic brain structures and the insula that support emotion expression (Ochsner, Silvers, \& Buhle, 2012). While limbic brain structures seem to be more involved in initial threat monitoring, the insula response represents current levels of subjective anxiety (Ochsner et al., 2012; Somerville et al., 2013) (previous chapter). Until today, there are only two fMRI studies investigating cognitive reappraisal in patients with anxiety disorders. In the first study, patients with social anxiety disorder were compared to healthy controls regarding their ability to reappraise autobiographic negative self-beliefs. The study found that engagement of dorsolateral prefrontal cortex (dIPFC) during reappraisal was delayed in patients, and this delay was the only brain indicator predicting increased anxiety (Goldin, Manber-Ball, Werner, Heimberg, \& Gross, 2009). Another study comparing patients with posttraumatic stress disorder with healthy controls, also found that patients showed an attenuated response of the lateral prefrontal cortex during reappraisal (New et al., 2009). The authors concluded that resilience may depend on the ability to focus intensively on the negative emotional response while concurrently engaging prefrontal brain systems to cope with negative emotion (New et al., 2009). Finally, a third study, which investigated regulation of conditioned fear in healthy participants as a model of anxiety disorders, found that activation levels in dIPFC were correlated with regulation success (Delgado, Nearing, Ledoux, \& Phelps, 2008). Therefore, all studies which specifically investigated cognitive reappraisal in the context of anxiety regulation, support a crucial role of the dIPFC in effortful anxiety regulation.

Until today, the approach in fMRI neurofeedback training for patients with emotional disorders has been emotion regulation training through learning voluntary control of activation in regions of the emotion network. In depressed patients a positive approach has been implemented, involving the up-regulation of brain regions of a network supporting positive emotions. This approach avoids the caveats of a downregulation instruction, which may lead patients to attenuate any emotional response independent of its valence. However, so far such a positive approach has not been developed for anxiety disorders, and there is strong evidence that a negative approach, teaching anxiety patients how to attenuate (anxious) feelings in order to feel safe, may be counter-productive. It is known from a long research line that attenuation, avoidance and suppression of anxiety has adverse long-term effects in patients (Amstadter, 2008; 
Barlow, 2002). There is also neuroscientific evidence showing that emotional networks need to be activated during extinction learning (Sehlmeyer et al., 2009), which is the mechanism for positive long-term changes in patients (Barlow, 2002). Therefore, especially patients should not be encouraged to simply attenuate activation levels in regions involved in anxiety processing, as this may prevent improvement in the long run. In order to avoid that patients simply learn how to suppress anxiety, a neurofeedback training that focuses on coping with anxiety, rather than reducing it, may paradoxically have a larger anxiety-reducing effect in the long run. Therefore, a positive approach to a neurofeedback training for anxiety patients may be instructing them to experience feelings of anxiety, while at the same time providing them with neurofeedback to enhance the learning of coping strategies.

We therefore implemented a neurofeedback training that provides participants with clinical levels of spider phobia with neurofeedback from dIPFC, in order to inform them about their engagement in cognitive reappraisal. To improve the face validity of this approach, we provided dual feedback, displaying both the activation level of the right insula (as part of the emotion network), as well as the activation in the dIPFC (as part of the regulatory network) to the participants, but instructing participants to shape their regulation strategy by focusing on the feedback from the regulatory network. To investigate if neurofeedback would enhance the efficacy of anxiety regulation, we compared this group to a patient control group in a randomized design with blinding of participants. Control participants received the same instruction and training but were not provided with neurofeedback information. We hypothesized that neurofeedback would enhance the efficacy of learning to regulate anxiety by cognitive reappraisal.

\section{Methods}

\section{Participants and Ethics Statement}

Participants were eighteen females (table 1), who were recruited by public advertisement at Maastricht University, screened for high spider fear [Spider Phobia Questionnaire (SPQ) Score $\geq 14$, (Klorman, Weerts, Hastings, Melamed, \& Lang, 1974)], and diagnosed with spider phobia according to the criteria of The Diagnostic and Statistic Manual of Mental Disorders DSM-IV TR (American Psychiatric Association, 2000). All were free of psychotropic medication and were not affected by 
Table 1. Characteristics of study participants.

\begin{tabular}{|l|l|l|l}
\hline $\begin{array}{l}\text { Variables (mean } \\
\text { +l- SD) }\end{array}$ & $\begin{array}{l}\text { Control } \\
\text { group }\end{array}$ & $\begin{array}{l}\text { Neurofeedback } \\
\text { group }\end{array}$ & p-value \\
\hline Gender (female) & $\mathrm{n}=9$ & $\mathrm{n}=9$ & \\
\hline Age & $21.7(2.1)$ & $20.7(1.2)$ & 0.23 \\
\hline Duration (years) & $15.0(3.0)$ & $14.0(1.9)$ & 0.42 \\
\hline ERQ & $30.1(3.5)$ & $29.1(2.8)$ & 0.52 \\
\hline SPQ & $19.2(2.9)$ & $19.3(3.4)$ & 0.94 \\
\hline FSQ & $90.0(14.8)$ & $91.2(10.9)$ & 0.84 \\
\hline SBQ & $56.2(10.5)$ & $54.9(7.9)$ & 0.77 \\
\hline
\end{tabular}

Participants were 18 spider phobic females, who were matched regarding age, individual differences in everyday use of reappraisal strategies (ERQ: Emotion Regulation Questionnaire), and the Spider Phobia Questionnaire (SPQ) score for fear of spider. All were diagnosed with spider phobia according to the Structured Clinical Interview DSM IV (SCID), and administered the Fear of Spider questionnaire (FSQ), and Spider Belief Questionnaire (SBQ). Both groups reported a similar duration since the onset of symptoms, and similar levels of spider fear.

current or previous other neuropsychiatric comorbidity as evaluated by means of a structured clinical interview [Mini International Neuropsychiatric Interview, MINI, (Sheehan et al., 1998)]. Allocation of participants to the control and neurofeedback group was balanced for the factors spider fear (SPQ score), age, and individual differences in self-reported use of reappraisal strategies [Emotion Regulation Questionnaire, ERQ, (Gross \& John, 2003)], using a restricted randomization procedure shown to be efficient for small sample sizes [sequential balancing, (Borm, Hoogendoorn, den Heijer, \& Zielhuis, 2005; Scott, McPherson, Ramsay, \& Campbell, 2002)]. Participants received a small financial compensation ( $8 € /$ hour), and gave their written informed consent prior to the experiment that was conducted in conformity with the Declaration of Helsinki and approved by the local Medical Ethics Committee at Maastricht University.

\section{General procedure}

Each participants attended four sessions, the screening session, the MRI scanning session, and two assessment sessions two weeks, and three months later. During screening, all participants were administered the Fear of Spider Questionnaire [FSQ, 
(Szymanski, 1995)], an instrument with high test-retest stability and internal consistency used to evaluate treatment changes in spider fear (Muris \& Merckelbach, 1996). Additionally, they completed the Spider Belief Questionnaire [SBQ, (Arntz, Lavy, Van den Berg, \& Van Rijsoort, 1993)], which was specifically designed to measure the changes in beliefs held by spider phobics. This instrument was used as a more direct measure of the changes achieved by cognitive reappraisal. Both questionnaires were administered a second time directly after scanning, and again at the two later assessment sessions.

On the MRI scanning day all participants were informed that the goal of the study was investigating the efficacy of cognitive reappraisal for fear regulation, and only the experimental group was told that they were participating in a neurofeedback study. All participants started with half an hour of instruction and practice. The instructor guided the participants to perform an active reinterpretation of the situation by "focusing on calming aspects" instead of "engaging in anxiety provoking thoughts", or "distancing themselves mentally", and explained that reappraisal may have a favorable long term effect on anxiety. Participants were asked to practice replacing their anxiety provoking, and distancing thoughts triggered by spider photographs with more anxiolytic reappraisal thoughts during the MRI session. They were suggested four different reappraisal strategies: 1) focusing on the esthetics of the spider, 2) focusing on its powerlessness, 3 ) changing its connotation by humanizing it, 4) changing its context by imagining approaching it in a safe environment. They were explained that these strategies were developed in order to counter-act some of the most common negative beliefs commonly hold by spider phobics (Arntz et al., 1993), and draw the focus to the safety of the situation, rather than escaping from it. Each participant was then invited to write down their own personal credible version of each reappraisal strategy. Finally, participants were familiarized with the procedure in the MRI scanner, and asked to rehearse the different reappraisal strategies out aloud during eight practice trials (regulate trials) under supervision. To allow participants to experience the impact of the coping strategy, as well as measure changes in anxiety over time, they were asked to refrain from changing their thoughts, letting them occur spontaneously, in half of the trials (watch trials).

The neurofeedback group was then familiarized with the feedback display, and explained that they would receive feedback from two brain regions. First, from a brain region involved in anxiety processing, indicating their level of anxiety, and second, from 
an area involved in the generation of reappraisal thoughts, indexing their engagement. Neurofeedback participants were instructed that they should continuously adapt their reappraisal strategies based on the feedback from the 'reappraisal region' during the experiment. They were asked to select the strategies which generated the high activation levels in this region. They were further explained that the feedback from the 'anxiety region' was for observation, and that high activation in this region, and experiencing high anxiety levels would generally not be harmful, and an essential part of the regulation process. The control group participants received the same instruction on experiencing high anxiety levels, and were asked to adapt their reappraisal strategies throughout the experiment based on their own insight. All participants were reminded that they could stop at any time, and asked to refrain from any movements in the scanner. After the instruction, all completed the Questionnaire of Current Motivation [QCM, (Rheinberg, Vollmeyer, \& Burns, 2001), which was administered to measure individual differences in the motivation to participate in the imaging session. Finally, to assess treatment acceptance, after the imaging session everyone was asked to rate how much would like to come back for another session.

\section{Procedure MRI session}

To accustom the participants to the scanner environment each imaging session started with a five minute long anatomical imaging run, during which participants were shown silent cartoons to reduce anticipatory anxiety. After the anatomical scan four functional imaging runs of eleven minute duration followed. First a localization run, used to functionally define the target regions, from which the feedback would be derived for the neurofeedback group, then three experimental runs, during which the neurofeedback group received feedback. During all functional runs participants performed the same task, letting their thoughts and feelings occur spontaneously during watch trials, and applying the reappraisal strategy during regulate trials. They were instructed to use the reappraisal strategy throughout the whole $12.5 \mathrm{~s}$ during which the spider photograph was presented. The presented spider photographs were selected from a previously developed and validated parametric spider picture set (previous chapter), each stimulus being presented once per condition during the experiment. Each functional run consisted of 16 trials, presented in blocks of four (e.g., 4 watch, 4 regulate, 4 watch, 4 regulate), counterbalancing the order between functional runs and participants. 
The task was programmed, and presented using Presentation software package (Version 16, Neurobehavioral Systems Inc., Albany, CA, USA). Each trial started with a $1.5 \mathrm{~s}$ cue (pictogram for watch or regulate), followed by $1 \mathrm{~s}$ fixation, and $12.5 \mathrm{~s}$ presentation of the spider photograph (figure 1). Participants could then indicate their subjective anxiety on a 5 point Likert scale from $0=$ 'not fearful at all' to $4=$ 'extremely fearful' using a button box, followed by a fixation cross until the $2.5 \mathrm{~s}$ presentation of the neurofeedback $6.25 \mathrm{~s}$ after the picture. Between trials there was a jittered resting period of $8.75+/-2.5 \mathrm{~s}$. During the localization run, and during watch trials, the neurofeedback group saw the same visually matched break display as the control group. Participants of the neurofeedback group therefore only received feedback regarding their performance during regulate trials. As previous research had shown that intermittent neurofeedback may be more effective in promoting learning than continuous neurofeedback (Johnson et al., 2012), and to avoid cognitive overload, and distraction during reappraisal, the feedback was presented delayed at the end of the trial, rather than continuously. Finally, to keep participants challenged throughout the experiment, the spider photographs were presented in a step-wise procedure, selected to provoke four increasing anxiety levels during the four functional runs (figure 2) (previous chapter).

\section{MRI imaging}

The images were acquired at Maastricht Brain Imaging Centre (Maastricht University) on a $3 \mathrm{~T}$ scanner (Tim Trio/upgraded to Prisma Fit, Siemens Healthcare, Germany), equipped with a 32/64 channel head coil. The participants were placed comfortably in the scanner and their heads were fixed with foam cushions to minimize motion. Functional images were acquired with a repeated single-shot echo-planar imaging (EPI) sequence that was optimized for imaging of subcortical and prefrontal areas by reducing echo time to TE $=25 \mathrm{~ms}$ (Domsch et al., 2013; Morawetz et al., 2008; Weiskopf, Hutton, Josephs, Turner, \& Deichmann, 2007), minimizing slice thickness to $2.5 \mathrm{~mm}$ with 20\% gap (Robinson, Windischberger, Rauscher, \& Moser, 2004; Weiskopf et al., 2007), and using a tilted slice angle of $25-30^{\circ}$ (Deichmann, Gottfried, Hutton, \& Turner, 2003). Repetition time was TR $=1250 \mathrm{~ms}$, adjusted flip angle $\mathrm{FA}=67^{\circ}$, and inplane resolution $3 \times 3 \mathrm{~mm}$, resulting in a voxel-size of $3 \times 3 \times 3 \mathrm{~mm}^{3}$. For the Tim Trio a field of view FOV $=210 \times 210 \mathrm{~mm}$, and matrix size $70 \times 70$ was used with bandwidth 1400 $\mathrm{Hz} / \mathrm{Px}$, grappa acceleration factor 2, and multiband acceleration factor 2, imaging 44 

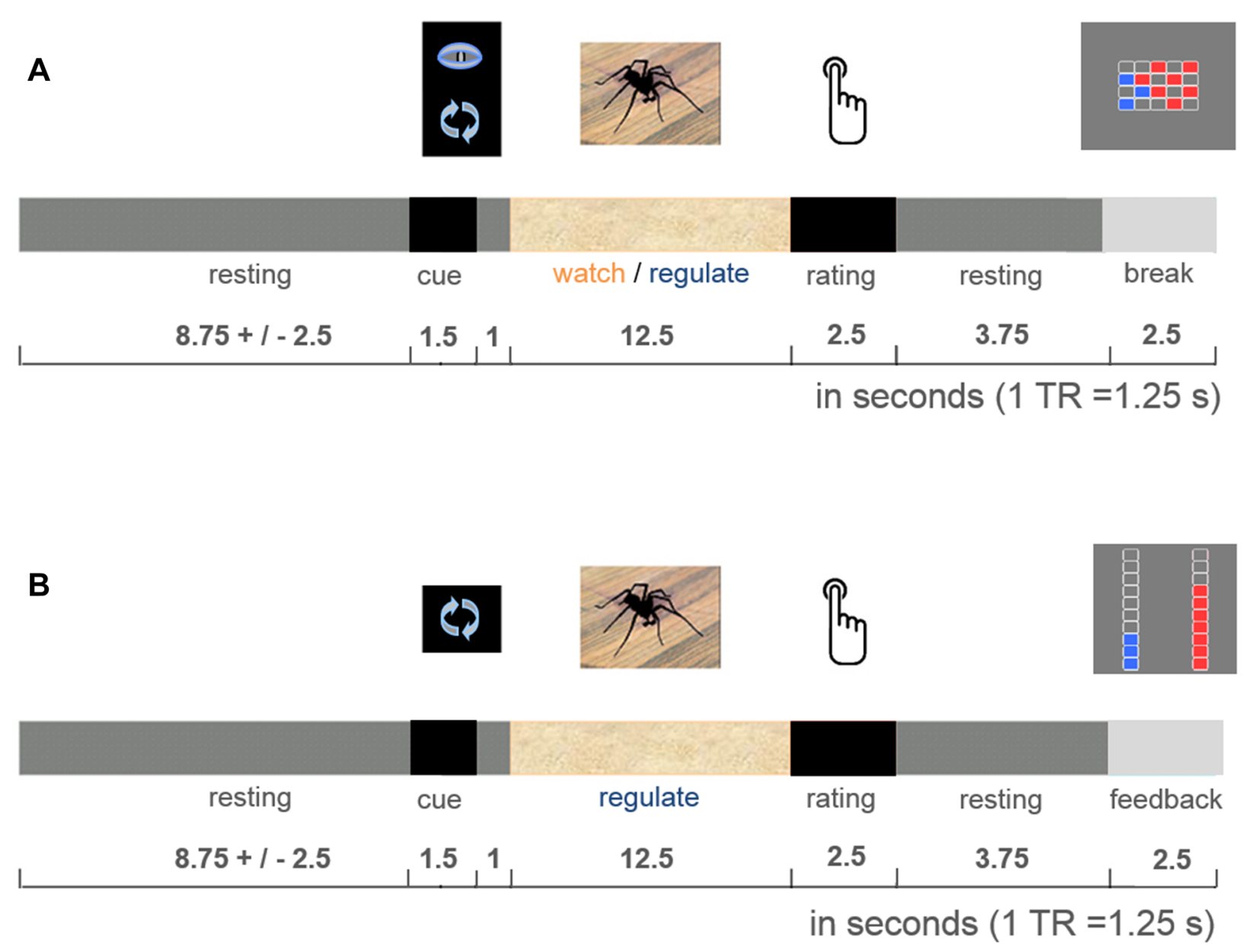

Figure 1. Experimental trial during scanning.

Each trial started with a cue, followed by a short fixation, and the presentation of the spider photograph (A, B). Depending on the kind of cue, participants were asked to either let their thoughts occur spontaneously or apply the reappraisal strategy during picture presentation. They then rated their subjective level of anxiety experienced during the current trial on a Likert scale, followed by a fixation cross until the presentation of the neurofeedback. During experimental runs in the neurofeedback group, the feedback was presented at the end of each regulate trial $(B)$. During watch trials, the localization run, and regulate trials of the control group, a visually matched break display was shown (A). The neurofeedback was presented delayed to avoid distraction, cognitive overload, and promote learning.

slices per volume. After the Prisma fit upgrade these parameters were changed to a field of view FOV = 192x192 mm, matrix size 64x64, bandwidth $1698 \mathrm{~Hz} / \mathrm{Px}$, and grappa acceleration factor 2 , imaging 24 slices per volume. This ensured full coverage of the prefrontal cortex, subcortical structures and occipital cortex, while lacking coverage of the parietal cortex. During all functional runs, heart rate was monitored 


\section{Neurofeedback presented from experimental run 1}

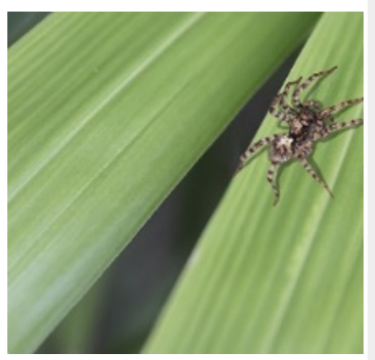

Localization run

anxiety level 1

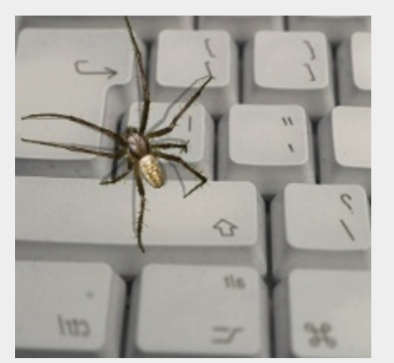

Experimental runs

(1)

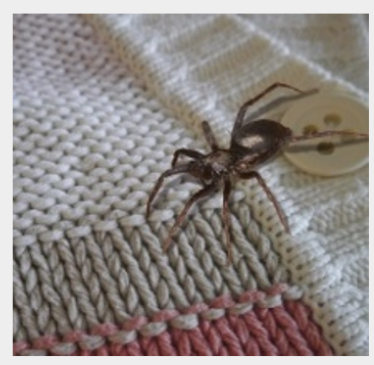

(2)

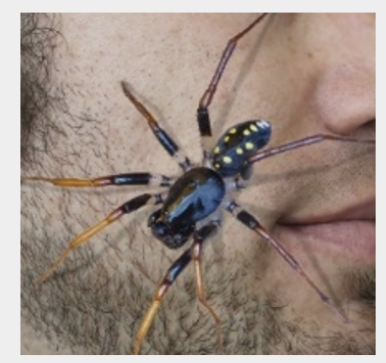

(3)

anxiety level 2

anxiety level 3

anxiety level 4

time

Figure 2. Parametric spider stimuli.

Spider photographs designed to provoke different anxiety levels were selected from a previously developed and validated parametric spider picture set (previous chapter). The perceived proximity to the spider was manipulated through systematic variation of the spider's size, posture, and the context it was placed in, as depicted in the four exemplary photographs. Across functional runs, the provoked anxiety level was increased step-wise to keep participants challenged throughout the experiment.

using the scanner's standard MRI-compatible pulse oximeter, to measure changes in heart rate, the expected autonomous response in spider phobia (Prigatano \& Johnson, 1974; Sarlo, Palomba, Angrilli, \& Stegagno, 2002). Breathing rate was monitored with a standard breathing chest band. Due to technical problems during recording all physiological data was only stored and analyzed for the first 5 minutes of each 11minute functional run. Anatomical images were collected with a 3D MPRAGE sequence: $\mathrm{TR}=1900 \mathrm{~ms}, \mathrm{TE}=2.52 \mathrm{~ms}, \mathrm{FA}=9^{\circ}, \mathrm{FOV}=256 \times 256 \mathrm{~mm}^{2}, 176$ slices, voxel size $1 \times 1 \times 1 \mathrm{~mm}^{3}$, with duration 4:26 minutes.

\section{Real-time MRI data analysis}

Anatomical images were processed using BrainVoyager QX (BVQX, Version 2.7, Brain Innovation B.V., Maastricht, The Netherlands), and loaded into the real-time data 
analysis software TurboBrainVoyager (TBV, Version 3.2 beta, Brain Innovation B.V., Maastricht, The Netherlands). Analysis of all functional data was performed in TBV. After discarding the first four volumes of each functional run due to T1 saturation effects, the data was automatically aligned to the anatomical scan, and pre-processed in real-time using intra-session 3D rigid-body motion correction, and drift confound predictors. An online voxel-wise general linear model (GLM) was computed, modelling the two task predictors (watch, regulate), as well as predictors for events of no interest (cue watch, cue regulate, rating watch, rating regulate, break display, feedback display), convolving them with a standard two-gamma hemodynamic response function. Prior to the definition of the target regions, the data from the localization run was high-pass filtered with a GLM Fourier basis set ( 2 cycles), and thresholded at $t=$ 3 , with an additional cluster threshold of four significant voxels. For both the control group and the neurofeedback group, the target regions were defined based on the functional contrast watch versus resting to individually localize the right insula (as an anxiety region), and regulate versus resting to individually localize the left dIPFC (as a reappraisal region). Anatomical target coordinates for the anxiety region in the right insula $(x=37, y=11, z=3)$, and for the reappraisal region in the left dIPFC $(x=-43$, $y=28, z=30$ ) were specified according to the literature (Delgado et al., 2008; Etkin \& Wager, 2007). The regions were defined unilaterally, as brain activation patterns during reappraisal are often lateralized (Delgado et al., 2008; Ochsner et al., 2012). For each participant the closest significant clusters to the respective target coordinates were selected by determining individual $t$-threshold values revealing activity (to a minimum of $t=2$, if necessary). During the following experimental runs, the feedback information for the neurofeedback group was computed by a custom-made TBV plugin, which calculated the average activation increase within the target regions during the last 10 $\mathrm{s}$ of the presentation of the spider photograph, relative to a $7.5 \mathrm{~s}$ period previous to the picture onset (figure 3). For each participant, the thermometer display was adjusted to their individual blood-oxygen-level dependent (BOLD) activation level, by setting the maximum percent signal change (PSC) of the thermometer display to two times the average PSC reached during the localization run within the respective target regions.

\section{Subjective ratings analysis}

The subjective anxiety ratings collected during the imaging session were analyzed in SPSS Statistics (IBM SPSS Statistics 21; IBM Corporation, Armonk, NY, USA). To 
investigate group differences, they were submitted to a repeated measures GLM with linear contrasts, modelling the within factors task (watch, regulate), and functional run (localization run, experimental run 1, experimental run 2, experimental run 3), and group as a between factor. We tested for group differences across all runs, as well as during the localization run, and the experimental runs, separately. Effect sizes were estimated using partial eta squared (Cohen, 1973).

\section{BOLD PSC}

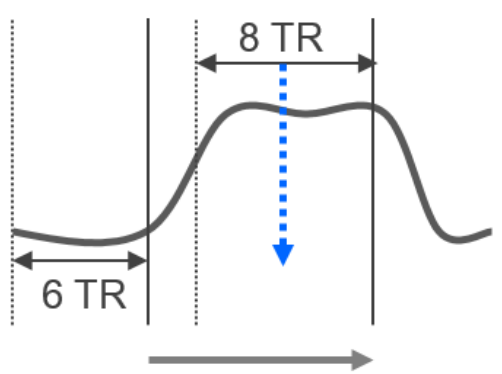

reappraise

(10 TR)

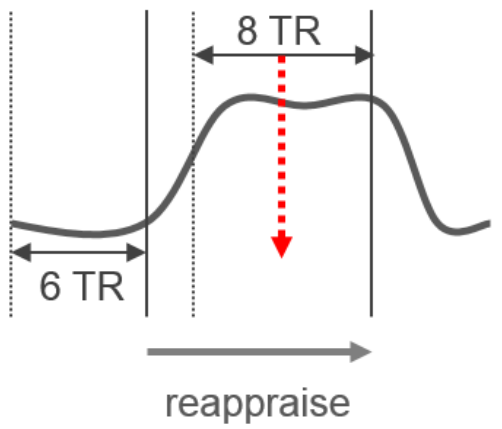

$(10 \mathrm{TR})$

\section{Thermometer display}

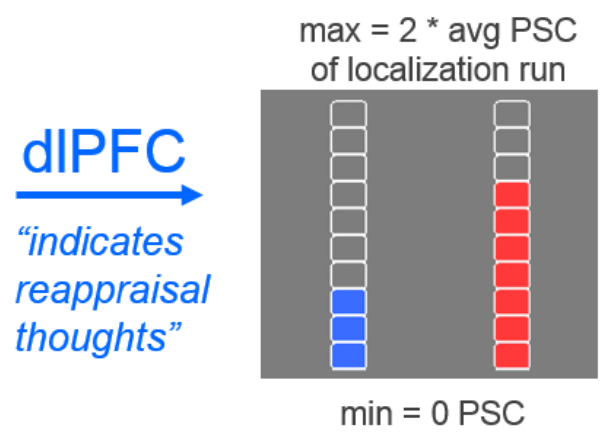

insula

"indicates anxiety"

$(1 \mathrm{TR}=1.25 \mathrm{~s})$

Figure 3. Neurofeedback computation and display.

The blood-oxygen-level dependent (BOLD) percent signal change (PSC) was computed using the activation increase during the last $10 \mathrm{~s}$ of the presentation of the spider photograph, relative to a $7.5 \mathrm{~s}$ period previous to the picture onset. For each participant the thermometer display was adjusted to their individual BOLD activation level, by setting the maximum PSC of the thermometer display to two times the average PSC obtained in the target ROI during the localization run. Neurofeedback participants were familiarized with the display prior to scanning, and instructed to continuously adapt their reappraisal strategies according to the feedback from the reappraisal region. They were asked to select the strategies, which generated the highest activation levels in this region. 


\section{Physiological data analysis}

The pulse and breathing rate from each participant were computed for the task conditions (watch, regulate), as well as the resting condition using a custom made MATLAB tool (R2010a; The MATHWORKS Inc., Natick, MA, USA). The data was analyzed in SPSS Statistics using a repeated measures GLM with the within factor task (watch, regulate, resting), and group as a between factor.

\section{Post-hoc MRI data analysis}

Functional and anatomical images were pre-processed in BrainVoyager QX, using the same parameters as during real-time processing. None of the participants moved more than $3.0 \mathrm{~mm} /$ degrees in any direction/rotation. All data was spatially normalized to Talairach space to enable a comparison between participants (Talairach \& Tournoux, 1988). A post-hoc region-of-interest analysis of the BOLD response in the target regions was performed to investigate group differences on a neural level. Beta estimates for the estimated BOLD activation (watch, regulate) were derived from all functional runs of all participants, based on the individually defined target regions. The BOLD activation of the target regions within the insula was investigated as a neural indicator of anxiety level, while the activation of the dIPFC target regions was interpreted as an index of engagement in the task. To statistically test for group differences the beta weights were submitted to the same statistical analysis as the subjective anxiety ratings.

Additionally, a whole-brain GLM analysis was conducted for the experimental functional runs to investigate group differences for task performance (watch, regulate), using a two-level random-effects GLM approach. For this purpose, the functional data was further pre-processed by spatial smoothing with an isotropic Gaussian kernel of 6 $\mathrm{mm}$ FWHM. Noise confounds were added to the GLM model, based on the three translational, and three rotational head motion parameters (Hutton et al., 2011; Weissenbacher et al., 2009), a localized estimate of the white matter signal to model scanner artifacts (Jo, Saad, Simmons, Milbury, \& Cox, 2010), and the ventricular signal to estimate physiological artifacts (Birn, Murphy, Handwerker, \& Bandettini, 2009). Statistical maps were thresholded using an initial voxel-threshold of $\alpha=0.05$ (Forman et al., 1995), and correcting for multiple comparisons using cluster-size thresholding with a cluster-level false positive rate of $\alpha=0.05$ (Forman et al., 1995; Goebel, Esposito, \& Formisano, 2006). 


\section{Pre-post assessment}

To evaluate between group differences in initial fear level, additional screening variables, and motivational scores were statistically evaluated using independent sample t-tests in SPSS Statistics. To evaluate long-term changes in spider fear, the scores from the four behavioral assessments were analyzed using a repeated measures GLM with linear contrasts, with the within factor time (screening, post-fMRI, two weeks, three months), and group as a between factor.

A

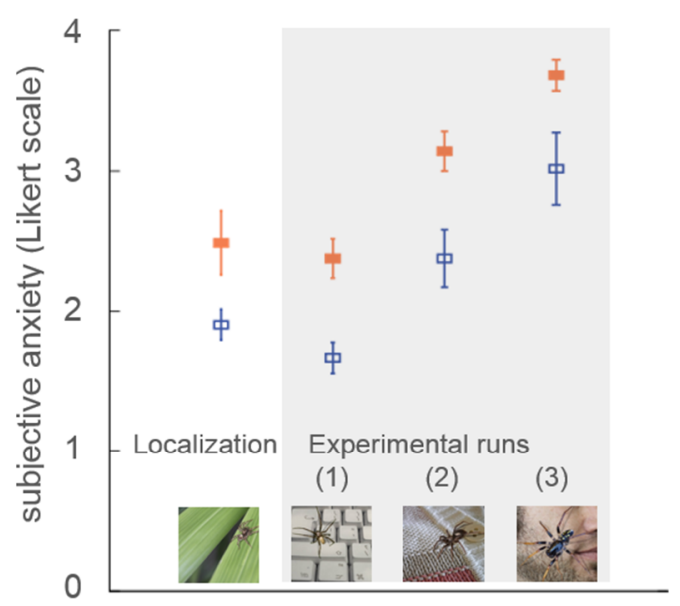

Control group
B

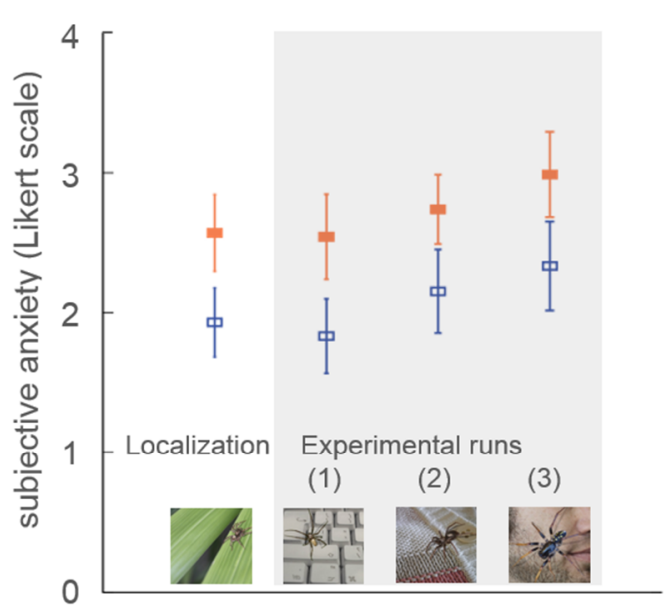

Neurofeedback group
C

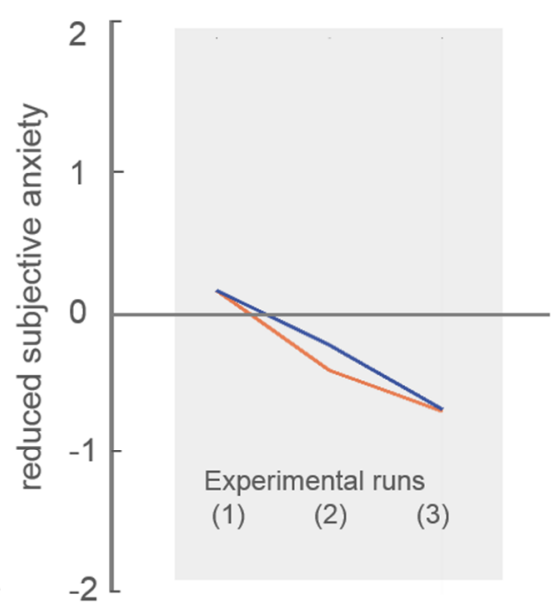

Group Difference

Figure 4. Subjective anxiety ratings during scanning.

Participants rated their subjective anxiety level on a Likert scale from $0=$ 'not fearful at all' to 4 = 'extremely fearful'. Throughout the MRI session, both groups had lower anxiety levels when applying the reappraisal strategy (blue), in comparison to when they watched the spider photographs and let their thoughts occur spontaneously (orange). While subjective anxiety levels during the initial localization run were similar in both groups (A, B), anxiety increased rapidly in the control group when the presented stimuli got more challenging $(\mathbf{A})$, and considerably less in the neurofeedback group (B). The group difference in subjective anxiety level, as calculated by subtracting the average ratings of the control group from the neurofeedback group within the three experimental runs, was not specific for the task condition (orange $=$ watch, blue $=$ regulate), but developed gradually over time starting only after participants had the first neurofeedback trials (C). 


\section{Results}

\section{Subjective ratings}

All participants completed the experiment as planned. The subjective anxiety ratings showed that throughout the MRI session both groups succeeded in down-regulating anxiety during regulate trials compared to watch trials $(F(1,16)=33.5, p<0.001$, $\eta_{p}^{2}=0.68$, figure 4). Importantly, there was no significant group difference during the initial localization run in the average level of anxiety $(p=0.84)$, or the ability to downregulate anxiety $(p=0.86)$. However, during the experimental runs, the two groups increasingly differed regarding their general anxiety level $\left(F(2,32)=6.4, p<0.01, \eta_{p}^{2}\right.$ $=0.29$ ). As the spider photographs became more anxiety provoking throughout the experimental runs, there was a strong, and significant increase of anxiety during both task conditions in the control group $\left(F(1,8)=33.3, p<0.001, \eta_{p}^{2}=0.81\right.$, figure 4a), while there was only a non-significant trend for an anxiety increase in the neurofeedback group $(F(1,8)=4.5, p=0.07$, figure $4 b)$. The neurofeedback group showed significantly reduced anxiety during both task conditions, an effect, which became more pronounced over time (figure 4c). Participants of both groups still continued to down-regulate anxiety successfully during regulate trials in comparison with watch trials $\left(F(1,16)=34.9, p<0.001, \eta_{p}^{2}=0.69\right)$.

\section{Physiological data}

The physiological data analysis showed no significant group difference for breathing $(p=0.36)$, or pulse rate $(p=0.45)$, and no differences between conditions (breathing: $p=0.26$; pulse: $p=0.36$ ). The average breathing rate of all participants was 18 breaths/minute, and the average pulse rate was 66 beats/minute (supplementary figure 1).

\section{MRI data}

In all participants the target regions could be defined based on the real-time fMRI data (figure 5). The average coordinates of the defined regions came close to the intended target coordinates, and the localization and size of the defined regions was similar across groups (supplementary tables 1,2 ). The BOLD response derived from the dIPFC target regions indicated that participants engaged in the regulation task as instructed. Both groups showed a robust up-regulation effect, defined as higher 
activation levels during regulate trials in comparison to watch trials, throughout the whole experiment $\left(F(1,16)=33.7, p<0.001, \eta_{p}^{2}=0.68\right.$, figure 6$)$. Separate analysis of the data from the localization run ( $p=0.35$, figure $6 \mathbf{a})$, and the experimental runs $(p=0.52$, figure $6 b, c)$, confirmed that there were no significant group differences

A
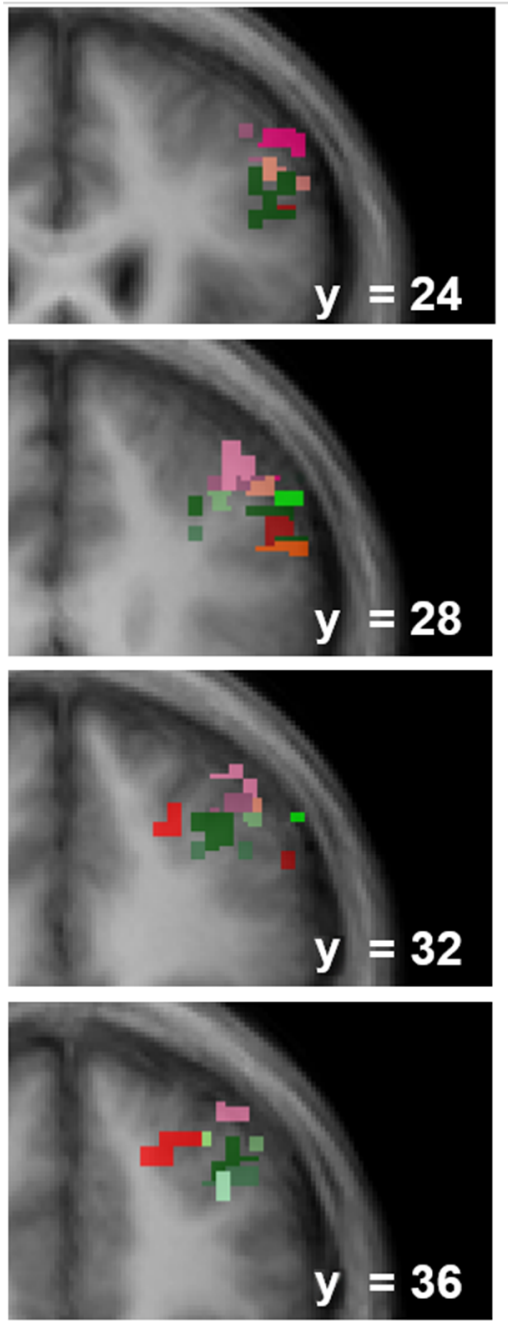

B
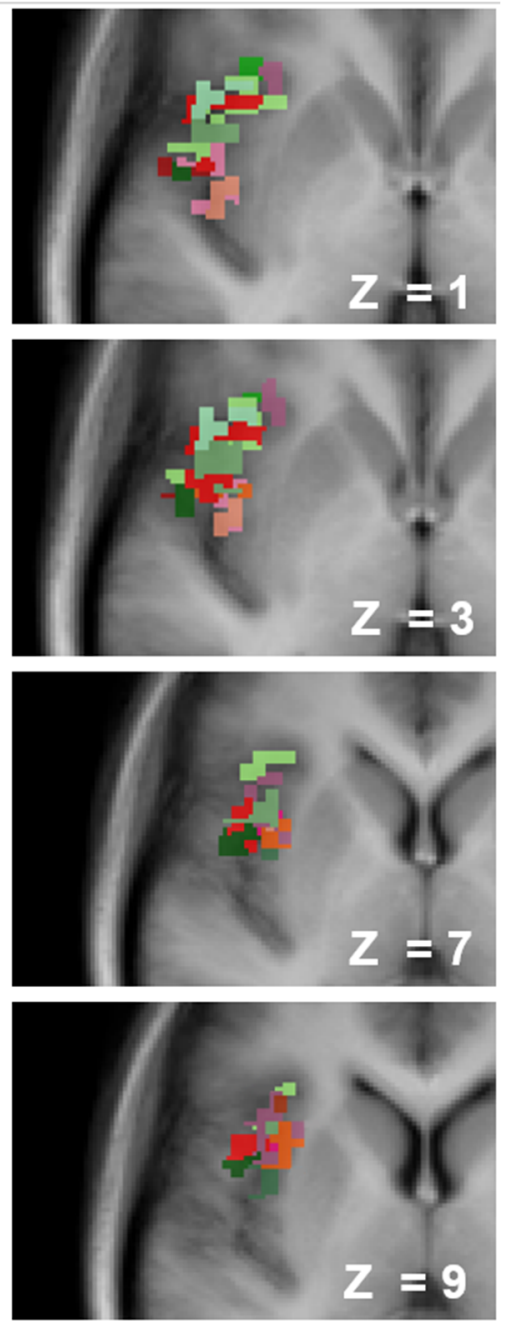

Figure 5. Localization of target regions.

The individual target regions, from which the feedback was derived for the neurofeedback group, and which were defined based on the localization runs for both groups, are depicted for the neurofeedback (red tints), and control participants (green tints). The regions in the dIPFC were defined by contrasting regulate trials versus resting, and selecting the closest cluster around the target coordinates $x=-43, y=28, z=30(A)$. The regions in the insula were defined by contrasting watch trials versus resting, and using the target coordinates $x=37, y=11, z=3(B)$. As depicted by this figure, the target regions were confined within the right insula and left dIPFC, and showed strong spatial overlap across groups. 
across time. Finally, there was an overall decrease of general activation level in the dIPFC with time $\left(F(1,16)=5.9, p<0.05, \eta_{p}^{2}=0.27\right)$ in both groups.

The beta estimates derived from the insula target region also indicated a decrease of general activation level across conditions in both groups $(F(1,16)=10.7$, $p<0.01, \eta_{p}^{2}=0.40$, figure 7 ), which was weakest in the regulate condition in the control group, and strongest in the watch condition of the neurofeedback group. Differences in activation level during the localization run did not reach significance in a direct group comparison ( $p=0.11$, figure $7 a$ ). During the experimental runs the neurofeedback group showed a down-regulation effect, defined as a reduced response in reappraisal trials in relation to watch trials, while the control group did not $(F(1,16)=$ $7.8, p<0.05, \eta_{p}^{2}=0.33$, figure $\left.7 \mathbf{b}, \mathbf{c}\right)$. This down-regulatory effect was significant in the neurofeedback group in the experimental runs $\left(F(1,8)=6.7, p<0.05, \eta_{p}^{2}=0.46\right)$, becoming larger in the second and third run, before the insula response was

A
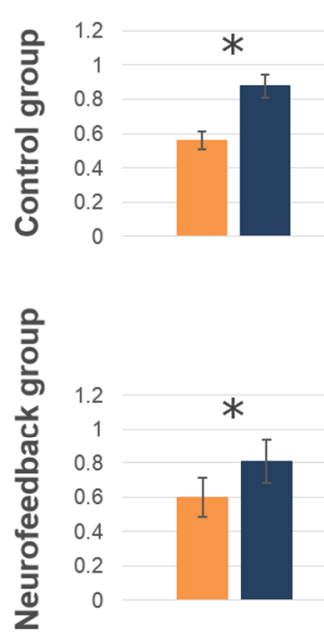

Localization
B
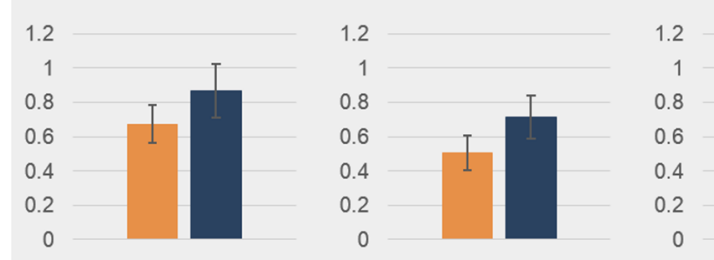

$*$
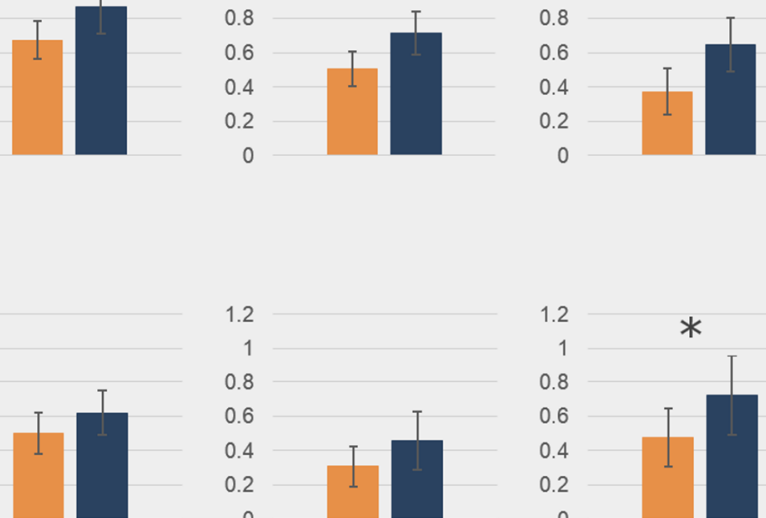

Experimental run 1
*

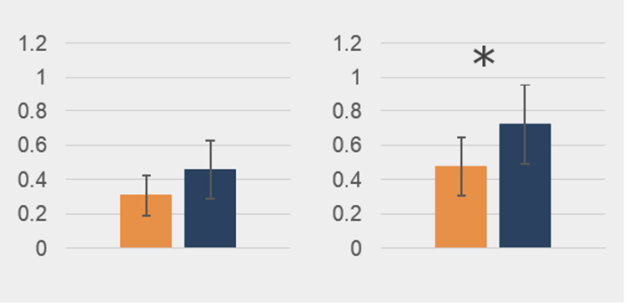

Experimental run 2

Experimental run 3

\section{C}

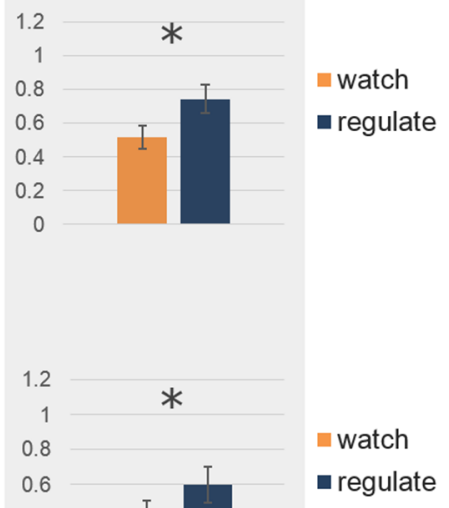

All experimental runs

\section{Figure 6. BOLD response in left dIPFC.}

The beta weights of the estimated BOLD response from the dIPFC target regions are depicted for the localization run (A), the experimental runs $(B)$, and the average of the experimental runs $(\mathbf{C})$. Both groups showed a robust up-regulation effect, with higher activation levels during reappraisal (blue) than watch trials (orange) throughout the experiment (significant results from post-hoc separate group analysis are marked with an asterisk for illustrative purposes). There were no significant differences between groups. 
completely attenuated in the fourth run. The same effect was non-significant in the control group ( $p=0.31$ ), disappearing completely in the second half of the experiment, when the activation levels during reappraisal became higher than in watch trials. The down-regulation effect therefore only manifested itself in the neurofeedback group, and, as for subjective anxiety level, this group difference increased over time. An exploratory whole-brain analysis confirmed an enhanced down-regulation of BOLD response by the neurofeedback group within a right-lateralized network of regions typically involved in anxiety expression, including the insula (supplementary table 2). The focus of this down-regulatory effect in the insula $(x / y / z=32 / 22 / 9)$ was in the vicinity of the target coordinates $(x / y / z=37 / 11 / 3)$ (figure 8 ).

A

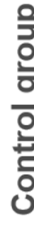

$-0.2$
B
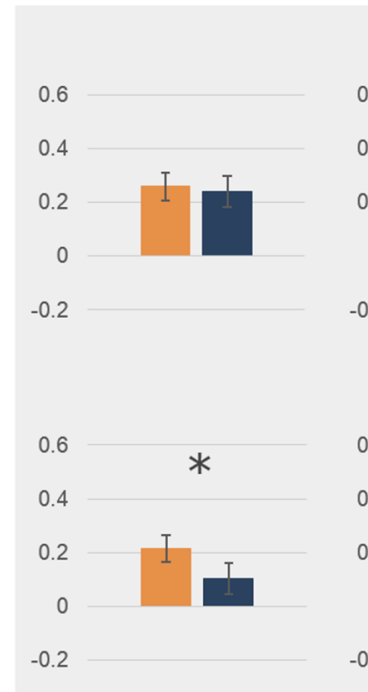

Experimental run 1

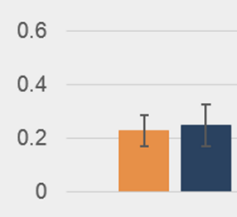

$-0.2$

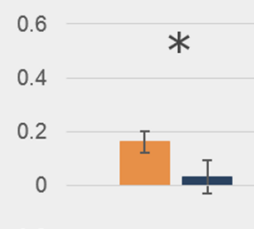

$-0.2$

Localization

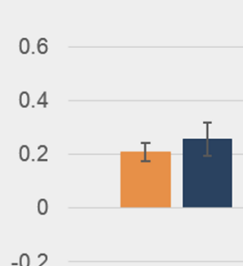

$-0.2$

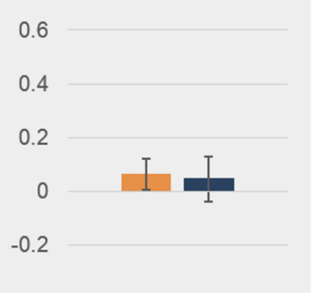

Experimental run 3
C

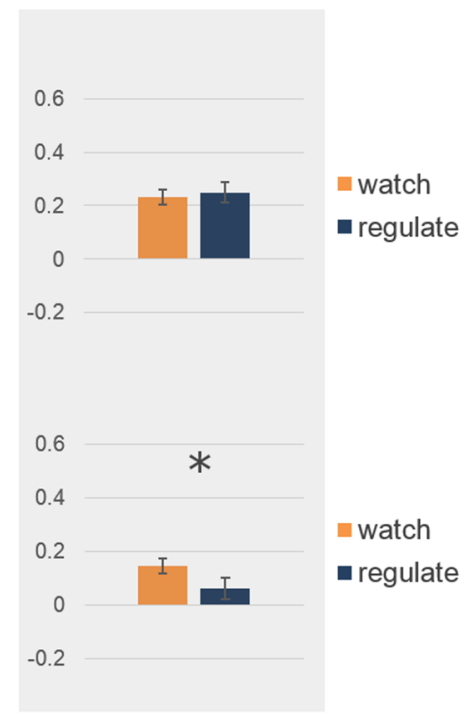

All experimental runs

\section{Figure 7. BOLD response in the right insula.}

The beta weights of the estimated BOLD response from the right insula target region are depicted for the localization run (A), separately for the experimental runs (B), and the average of the experimental runs (C). The slight differences between groups during the localization run did not reach significance in a direct group comparison (see text, significant results from posthoc separate group analysis are marked with an asterisk for illustrative purposes). During the experimental runs, the neurofeedback group showed significant down-regulation, characterized by a reduced response in reappraisal (blue) in relation to watch trials (orange), while the control group did not (C). In the neurofeedback group this effect was significant in the second and third run, before the insula response was completely attenuated in the fourth run. 
A

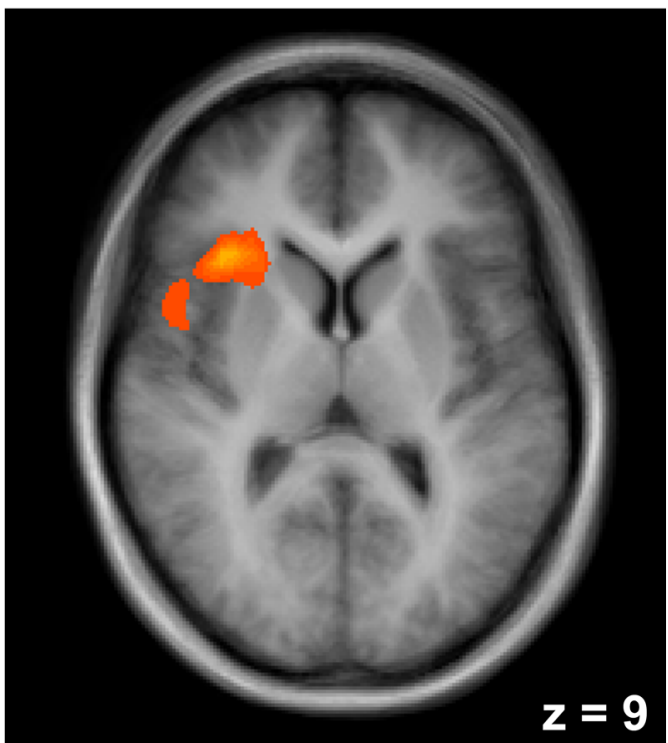

B

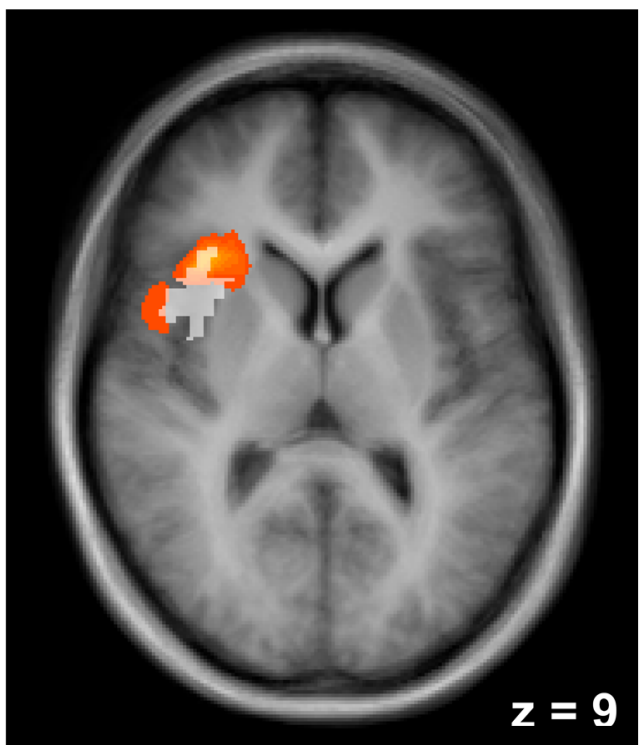

Figure 8. Whole brain analysis for down-regulatory effect.

The whole-brain random-effects GLM analysis computed based on the experimental runs showed a significant group difference for the down-regulatory effect in the insula $(p<0.05$, corrected at cluster level), defined as a reduced response during reappraisal, when contrasting regulate trials with watch trials $(\mathbf{A})$. The neurofeedback group showed a significantly greater reduced insula response during reappraisal than the control group. The focus of this effect $(x / y / z=32 / 22 / 9)$ was in the vicinity of the average coordinate of the defined target regions $(x / y / z=37 / 11 / 3)$, as indicated by overlaying the combined individual ROls on an exemplary slice (B). This further confirms that neurofeedback participants were more successful in downregulating than the control group.

\section{Pre-post assessment}

Assessment by questionnaires showed that all participants had similarly high levels of spider fear during screening (table 1). Both groups were highly motivated to participate after they received the instruction, and showed high willingness to return for a second session after completing the imaging session (table 2). Assessment of fear levels during the three-month period following scanning showed that fear decreased significantly in both groups (FSQ: $F(1,16)=23.0, p<0.001, \eta_{p}^{2}=0.59$, SBQ: $F(1,16)$ $=35.1, p<0.001, \eta_{p}^{2}=0.69$, figure 9). While the neurofeedback group had slightly lower anxiety levels than the control group, this group difference was not significant (FSQ: $p=0.47$, SBQ: $p=0.64)$. 
A

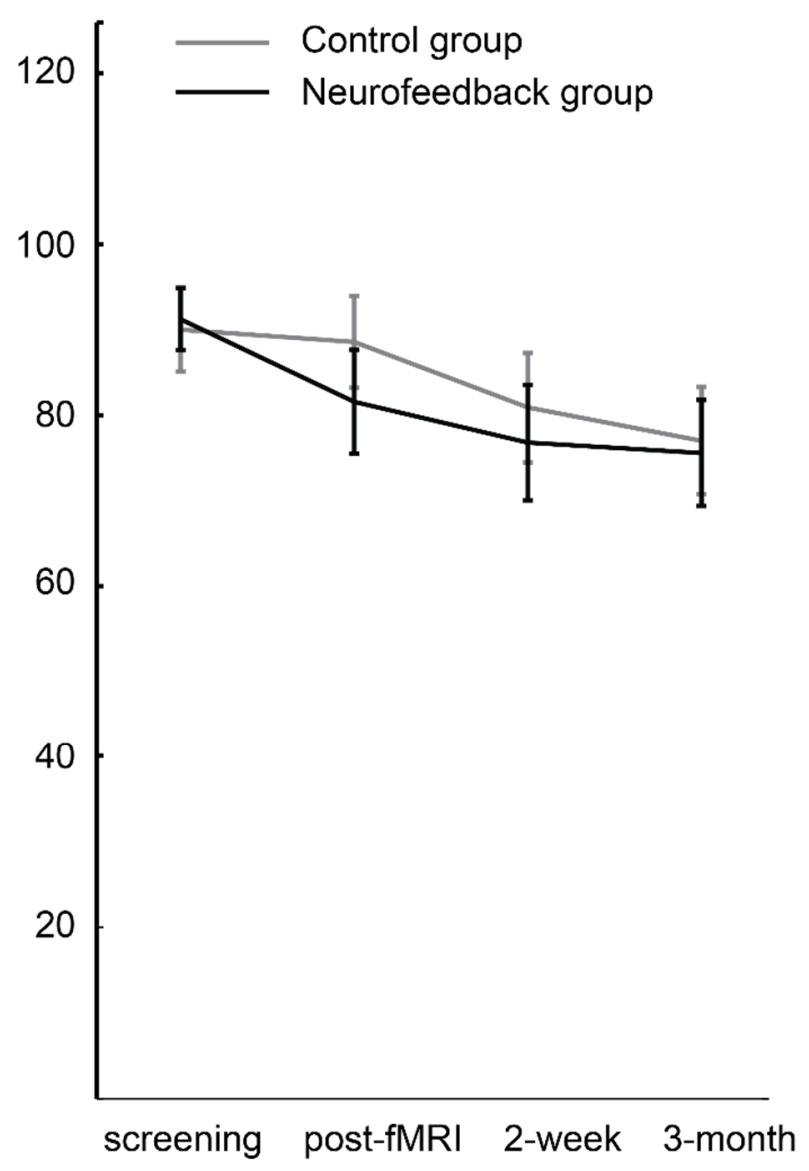

B

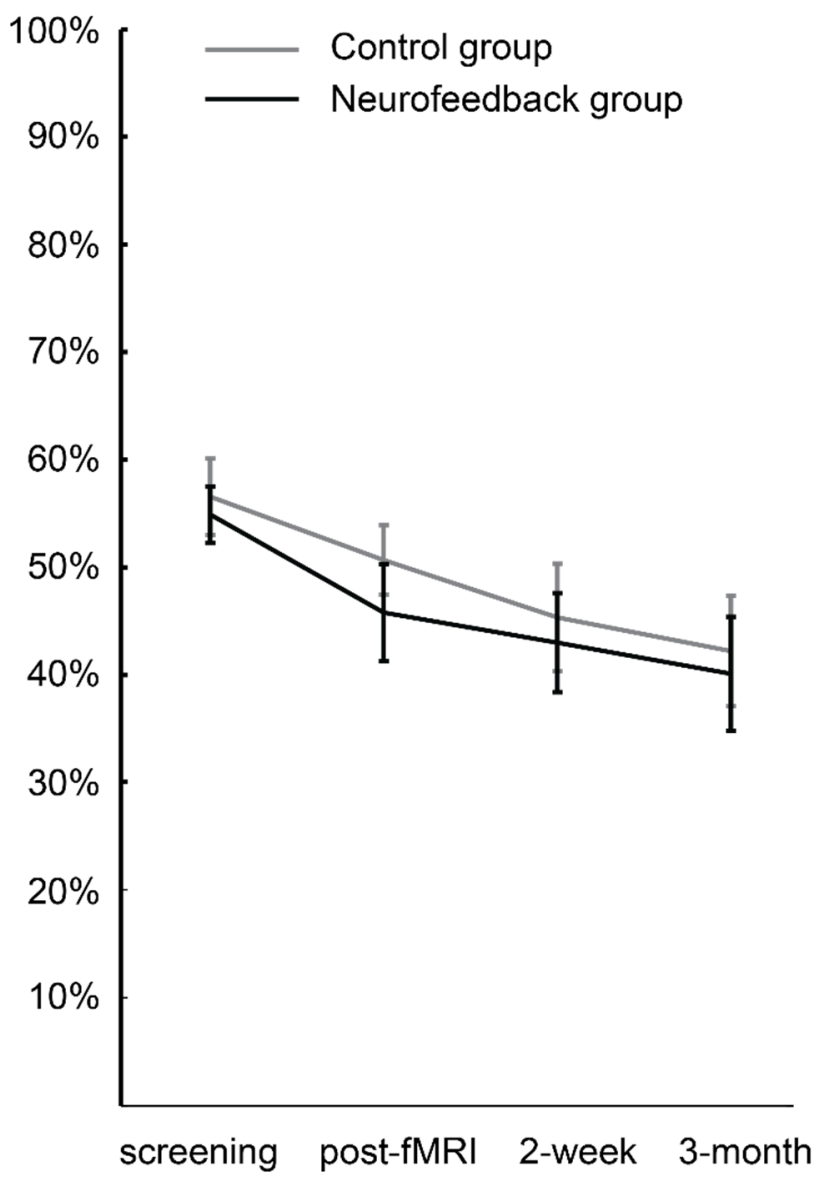

Figure 9. Long-term development of subjective anxiety.

The long-term development of subjective anxiety as assessed with the Fear of Spider Questionnaire (A), and the Spider Belief Questionnaire (B) is depicted. Participants had similarly high levels of fear during screening, and both groups showed a significant decrease of anxiety during a 3-month period after scanning. There were no significant group differences in the long term development of spider fear. 


\begin{tabular}{l|l|l|l|}
\hline \multicolumn{4}{|l}{ Table 2. Current Motivation and treatment acceptance. } \\
\hline $\begin{array}{l}\text { Variables (Mean } \\
\text { +/- SD) }\end{array}$ & $\begin{array}{l}\text { Control } \\
\text { group }\end{array}$ & $\begin{array}{l}\text { Neurofeedback } \\
\text { group }\end{array}$ & p-value \\
\hline QCM fear & $3.1(1.1)$ & $2.7(1.1)$ & 0.39 \\
\hline QCM challenge & $5.8(0.7)$ & $5.7(0.9)$ & 0.77 \\
\hline QCM interest & $5.4(1.0)$ & $5.3(0.7)$ & 0.79 \\
\hline QCM mastery & $5.4(0.5)$ & $5.7(0.7)$ & 0.46 \\
\hline $2^{\text {nd }}$ session? & $5.9(1.3)$ & $6.0(1.0)$ & 0.84 \\
\hline
\end{tabular}

The Questionnaire of Current Motivation (QCM) was administered prior to the scanning session, after participants had received the complete instruction. After the scanning session, participants were asked if they would be willing to come back for a second session. There were no significant differences between groups regarding any of the four different motivational factors (incompetence fear, perceived challenge, level of interest, mastery confidence), or willingness to continue with treatment. All motivational factors were measured on a Likert Scale (1-7).

\section{Discussion}

We hypothesized that neurofeedback would enhance the efficacy of learning to use cognitive reappraisal to cope with provoked anxiety. Overall, the presented results support this hypothesis, showing that neurofeedback participants indeed seemed to regulate anxiety more effectively at the end of the imaging session than control participants. In the second half of the session, neurofeedback participants coped better with provoked fear, as indicated by a much slower rise in subjective anxiety, showed a robust down-regulation effect in the insula, which was unique in the neurofeedback group, and a steady up-regulation effect for the dIPFC, indicating high task engagement. Finally, the long-term development of anxiety was positive.

The subjective anxiety rating results indicated that neurofeedback participants learned how to deal with the provoked anxiety more efficiently. While subjective anxiety levels during the initial localization run were similar in both groups, anxiety increased rapidly in the control group when the presented stimuli got more challenging, and considerably less in the neurofeedback group. This effect also transferred to the watch trials, which suggests a potential for learned regulatory strategies to operate implicitly. Importantly, the group difference in subjective anxiety level developed gradually over 
time, after participants had the first neurofeedback trials. This supports the hypothesis that increased control was indeed an effect of the provided neurofeedback. The analysis of the insula response also indicated that improved coping was related to better regulatory skills in the neurofeedback group. While there were slight initial differences between groups, the neurofeedback group showed a desired development over time (decrease of activity indicative of anxiety level), which the control group did not. In the neurofeedback group the down-regulatory effect in the insula became larger over time, while there was an opposite development during experimental runs in the control group. Interestingly, the analysis of the dIPFC data demonstrated that during the second half of the experiment, when anxiety levels were considerably lower in the neurofeedback group, both groups exhibited similar activation levels indicating equal engagement. These dIPFC/insular patterns of activity in the two groups suggest that the control participants were less efficient than the neurofeedback participants in reducing anxiety levels despite similar levels of control activity. In general, the brain pattern of the neurofeedback group at the end of the experiment was similar to activations patterns found after successful standard treatment in spider phobics, showing a reduction of the insula response with a relative increase of the attenuated dIPFC response (Etkin \& Wager, 2007; Hauner, Mineka, Voss, \& Paller, 2012; Schienle, Schäfer, Hermann, Rohrmann, \& Vaitl, 2007).

Finally, the long-term development of fear in the neurofeedback group was positive, with fear levels in the neurofeedback group remaining slightly below the level of the control group. In both groups a considerable, and consistent reduction of fear was achieved after this very short intervention, which included only 30 minutes of neurofeedback training. The fear reduction effect was, however, smaller than reported with traditional in-vivo exposure therapy (Arntz et al., 1993; Hauner et al., 2012; Muris \& Merckelbach, 1996), possibly because provoked anxiety levels were lower, thus limiting the potential for learning regulation. Interestingly, the willingness of participants to continue with treatment was very high, in comparison with standard exposure invivo treatment in spider phobia, which often has high dropout rates and low treatment acceptance (Pull, 2008). The proposed training may therefore potentially be extended, both in duration, and also by using new technical means, such as virtual reality environments, or 3D movie exposure. Also, the short session duration may be a possible explanation on why neurofeedback participants did not show a continued advantage over the control group. Previous research demonstrated that patients learn 
best, if they attended minimally two neurofeedback sessions (Linden et al., 2012; Scheinost et al., 2013). Importantly, the presented study demonstrated that fMRI neurofeedback trainings may be clinically relevant for training patients with high levels of anxiety, as initial fear levels were in the normal range for spider phobics (Arntz et al., 1993; Muris \& Merckelbach, 1996; Schienle et al., 2007).

A possible limitation in the interpretation of the effects might result from potential differences between the groups concerning motivation and confidence. In order to minimize the influence of motivation, the presented study was designed as a study with an non-neurofeedback control group, different to several previous studies which employed sham feedback control groups (DeCharms et al., 2005; Scheinost et al., 2013; Young et al., 2014). The rational of using a blinded non-neurofeedback control group was to optimally match both groups regarding motivational factors. The control participants in the presented study received a valid instruction, and underwent the exact same training as the experimental group, without being diverted by noninformative invalid feedback, as participants of a sham feedback control group might have been. The analysis of questionnaires, and fMRI data showed that both groups were indeed equally motivated throughout the experiment. In general, motivation was very high in both groups, the study had no dropout, and overall treatment acceptance was excellent, showing that the control group indeed received a training which was relevant and engaging. It therefore seems unlikely that the improved regulatory skills in the neurofeedback group were a mere result of motivational factors.

\section{Conclusions}

Overall, the presented results confirm for the first time that patients with clinical levels of anxiety can successfully use neurofeedback information to learn therapeutic techniques for anxiety regulation. The results generally support that neurofeedback may be a promising tool for treatment, as previously shown for other clinical populations (DeCharms et al., 2005; Haller et al., 2010; Linden et al., 2012; Scheinost et al., 2013; Subramanian et al., 2011; Young et al., 2014). Finally, the results show, that it may be promising to incorporate fMRI neurofeedback into ongoing developments for improving treatment for patients with anxiety disorders. 
A

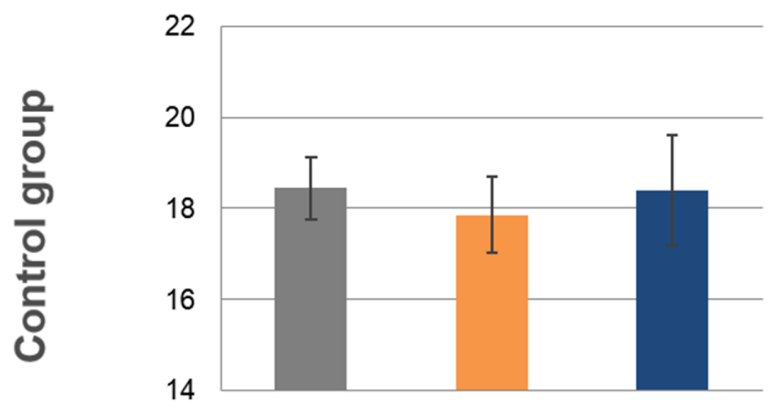

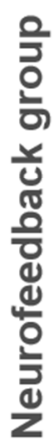

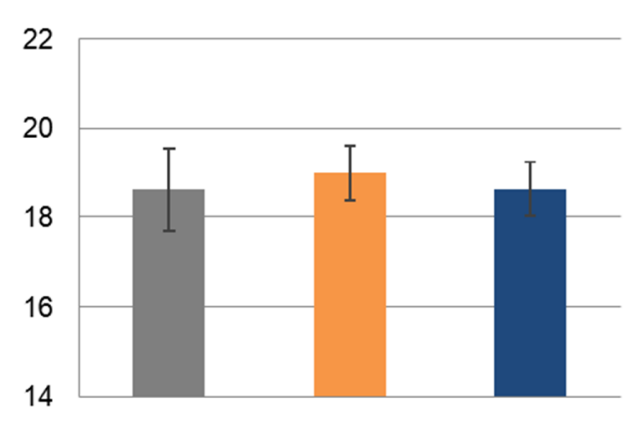

Breathing rate
B
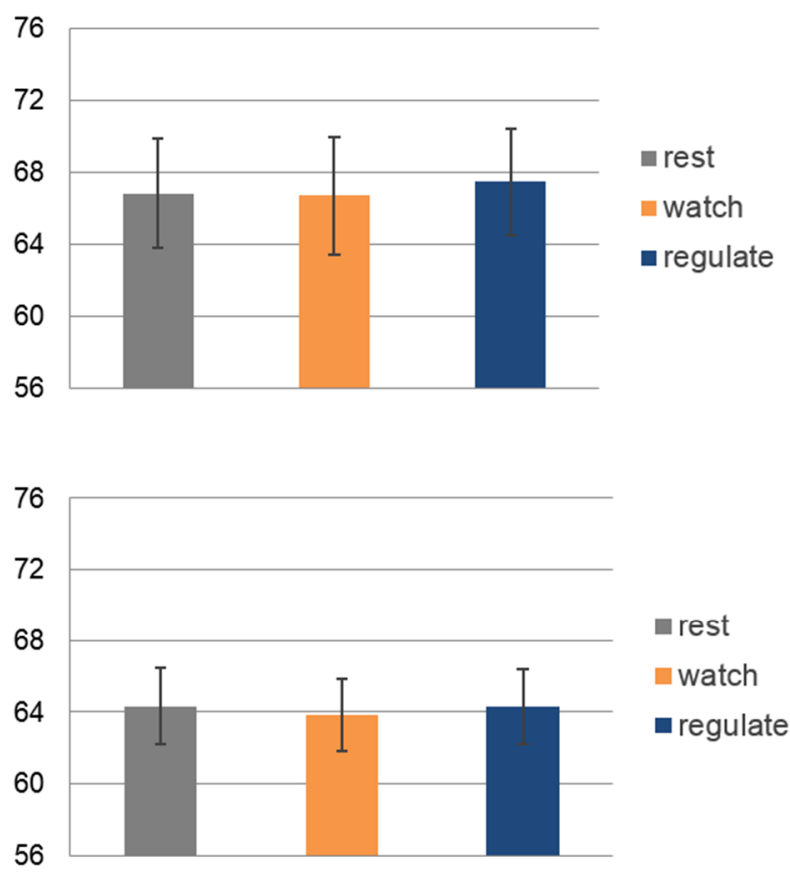

Pulse rate

\section{Supplementary figure 1. Pulse and breathing rates during scanning.}

The estimated pulse and breathing rate did not differ significantly between groups, or task conditions. The average breathing rate was 18 breaths/minute, and the average pulse rate was 66 beats/minute. 
Supplementary Table $1 \mathrm{~A}$. Talairach coordinates of dIPFC target regions.

\begin{tabular}{|c|c|c|c|c|c|c|c|c|c|}
\hline \multicolumn{5}{|c|}{ Control group } & \multicolumn{5}{|c|}{ Neurofeedback group } \\
\hline & $\mathbf{x}$ & $y$ & $\mathbf{z}$ & $\begin{array}{c}\text { Number of } \\
\text { functional voxels } \\
\left(3 \times 3 \times 3 \mathrm{~mm}^{3}\right)\end{array}$ & & $\mathbf{x}$ & $y$ & z & $\begin{array}{c}\text { Number of } \\
\text { functional voxels } \\
\left(3 \times 3 \times 3 \mathrm{~mm}^{3}\right)\end{array}$ \\
\hline S01 & -32 & 35 & 33 & 21 & S10 & -46 & 26 & 26 & 12 \\
\hline S02 & -47 & 16 & 28 & 17 & S11 & -48 & 23 & 39 & 10 \\
\hline S03 & -37 & 33 & 20 & 19 & S12 & -41 & 36 & 26 & 24 \\
\hline S04 & -48 & 27 & 33 & 6 & S13 & -42 & 30 & 24 & 6 \\
\hline S05 & -26 & 41 & 40 & 11 & S14 & -36 & 36 & 19 & 11 \\
\hline S06 & -33 & 35 & 30 & 11 & S15 & -33 & 45 & 28 & 12 \\
\hline S07 & -38 & 36 & 17 & 8 & S16 & -36 & 27 & 39 & 13 \\
\hline S08 & -44 & 24 & 29 & 25 & $\mathrm{~S} 17$ & -47 & 16 & 34 & 24 \\
\hline S09 & -34 & 39 & 30 & 11 & $\mathrm{~S} 18$ & -32 & 44 & 34 & 16 \\
\hline mean & -38 & 32 & 29 & 14 & mean & -40 & 31 & 30 & 14 \\
\hline SD & 7 & 8 & 7 & 6 & SD & 6 & 10 & 7 & 6 \\
\hline target & -43 & 28 & 30 & & target & -43 & 28 & 30 & \\
\hline
\end{tabular}

Supplementary Table $1 \mathrm{~B}$. Talairach coordinates of insula target regions.

\begin{tabular}{|c|c|c|c|c|c|c|c|c|c|}
\hline \multicolumn{5}{|c|}{ Control group } & \multicolumn{5}{|c|}{ Neurofeedback group } \\
\hline & $\mathbf{x}$ & y & $\mathbf{Z}$ & $\begin{array}{c}\text { Number of } \\
\text { functional voxels } \\
\left(3 \times 3 \times 3 \mathrm{~mm}^{3}\right) \\
\end{array}$ & & $\mathbf{x}$ & y & $\mathbf{z}$ & $\begin{array}{c}\text { Number of } \\
\text { functional voxels } \\
\left(3 \times 3 \times 3 \mathrm{~mm}^{3}\right)\end{array}$ \\
\hline S01 & 46 & 3 & 6 & 11 & S10 & 47 & 0 & -1 & 9 \\
\hline S02 & 37 & 20 & -1 & 17 & S11 & 37 & 10 & 10 & 10 \\
\hline S03 & 39 & -6 & 12 & 4 & $\mathrm{~S} 12$ & 43 & 2 & 2 & 17 \\
\hline S04 & 32 & 13 & 14 & 8 & S13 & 36 & 7 & 8 & 23 \\
\hline S05 & 40 & 15 & 5 & 25 & S14 & 39 & 1 & 4 & 7 \\
\hline S06 & 37 & 4 & 11 & 11 & S15 & 42 & 10 & 5 & 26 \\
\hline S07 & 42 & 10 & 4 & 22 & S16 & 33 & 16 & 9 & 26 \\
\hline S08 & 42 & 7 & 10 & 9 & S17 & 31 & 13 & 13 & 7 \\
\hline S09 & 42 & 18 & 3 & 6 & $\mathrm{~S} 18$ & 31 & 21 & 12 & 8 \\
\hline mean & 40 & 9 & 7 & 12 & mean & 38 & 9 & 7 & 15 \\
\hline SD & 4 & 8 & 5 & 8 & SD & 6 & 7 & 5 & 8 \\
\hline target & 37 & 11 & 3 & & target & 37 & 11 & 3 & \\
\hline
\end{tabular}

The coordinates and size (in functional voxels $3 \times 3 \times 3 \mathrm{~mm}^{3}$ ) of the individually localized target regions in the dIPFC and insula, from which the feedback was derived for the neurofeedback group, are listed for all subjects. The average coordinates came close to the target coordinates, and the size (e.g., number of voxels) of the defined regions was similar across groups. 
Supplementary Table 2. Regions showing a group difference for the contrast regulate vs. watch

Regions showing an increased difference in the neurofeedback group

\begin{tabular}{|c|c|c|c|}
\hline & & Brodmann's Areas & $x / y / z$ (no of voxels) \\
\hline \multirow{2}{*}{$\begin{array}{l}\text { Anterior cingulate } \\
\text { cortex }\end{array}$} & $\mathrm{L}$ & 24,32 & $-2 / 31 / 35 \quad(80)$ \\
\hline & $\mathrm{R}$ & 24,32 & $2 / 29 / 33 \quad(44)$ \\
\hline \multirow{2}{*}{$\begin{array}{l}\text { Dorsolateral prefrontal } \\
\text { cortex }\end{array}$} & $\mathrm{L}$ & & --- \\
\hline & $\mathrm{R}$ & 9 & $49 / 13 / 25(223)$ \\
\hline \multirow{2}{*}{$\begin{array}{l}\text { Ventrolateral prefrontal } \\
\text { cortex }\end{array}$} & $\mathrm{L}$ & & --- \\
\hline & $\mathrm{R}$ & 44,47 & 48/7/14 \\
\hline \multirow[t]{2}{*}{ Insula } & $\mathrm{L}$ & & --- \\
\hline & $\mathrm{R}$ & 13 & $32 / 22 / 9 \quad(203)$ \\
\hline
\end{tabular}

Regions showing a reduced difference in the neurofeedback group

---

Talairach coordinates of the most significant voxel and the number of significantly activated functional voxels $\left(3 \times 3 \times 3 \mathrm{~mm}^{3}\right)$ are reported.

The whole-brain random-effects GLM analysis based on the experimental runs demonstrated a significant group difference for the contrast regulate versus watch trials $(p<0.05$, corrected at cluster level), with a greater down-regulatory effect in the neurofeedback group than the control group. This effect was significant within a right-lateralized network of regions typically activated during anxiety expression, including the insula, where the target regions were defined. The focus of the effect in the insula $(x / y / z=32 / 22 / 9)$ was in the vicinity of the coordinates for defining the neurofeedback target regions $(x / y / z=37 / 11 / 3)$. 


\section{Acknowledgements}

We want to thank Andrea Hermann, Maren Bruns, and Andrea Wolf for their very helpful comments on writing the reappraisal instruction, Valentin Kemper and Federico De Martino for their generous support with optimizing fMRI data acquisition and preprocessing, and Michael Lührs, Joel Reithler, and Jan Zimmermann for providing custom data analysis tools. The authors gratefully acknowledge the support of the BrainGain Smart Mix Program of The Netherlands Ministry of Economic Affairs and The Netherlands Ministry of Education, Culture and Science (grant number: SSM06011). 


\section{References}

American Psychiatric Association. (2000). Diagnostic and Statistical Manual of Mental Disorders, Fourth Edition, Text Revision (DSM-IV-TR). Text (Vol. 1). Washington, DC: American Psychiatric Association. doi:10.1176/appi.books.9780890423349

Amstadter, A. (2008). Emotion regulation and anxiety disorders. Journal of Anxiety Disorders, 22(2), 211-21. doi:10.1016/j.janxdis.2007.02.004

Arntz, A., Lavy, E., Van den Berg, G., \& Van Rijsoort, S. (1993). Negative beliefs of spider phobics: A psychometric evaluation of the Spider Phobia Beliefs Questionnaire. Advances in Behaviour Research \& Therapy, 15(4), 257-277.

Barlow, D. H. (2002). True Alarms, False Alarms, and Learned (Conditioned) Anxiety. In D. H. Barlow (Ed.), Anxiety and its disorders: the nature and treatment of anxiety and panic (2nd ed., pp. 219-251). New York: The Guilford Press.

Birn, R. M., Murphy, K., Handwerker, D. A., \& Bandettini, P. A. (2009). fMRI in the presence of task-correlated breathing variations. Neurolmage, 47(3), 1092-104. doi:10.1016/j.neuroimage.2009.05.030

Borm, G. F., Hoogendoorn, E. H., den Heijer, M., \& Zielhuis, G. a. (2005). Sequential balancing: a simple method for treatment allocation in clinical trials. Contemporary Clinical Trials, 26(6), 637-45. doi:10.1016/j.cct.2005.09.002

Cohen, J. (1973). Eta-Squared and Partial Eta-Squared in Fixed Factor Anova Designs. Educational and Psychological Measurement, 33(1), 107-112. doi:10.1177/001316447303300111

DeCharms, R. C., Maeda, F., Glover, G. H., Ludlow, D., Pauly, J. M., Soneji, D., ... Mackey, S. C. (2005). Control over brain activation and pain learned by using realtime functional MRI. Proceedings of the National Academy of Sciences of the United States of America, 102(51), 18626-31. doi:10.1073/pnas.0505210102

Deichmann, R., Gottfried, J. ., Hutton, C., \& Turner, R. (2003). Optimized EPI for fMRI studies of the orbitofrontal cortex. Neurolmage, 19(2), 430-441. doi:10.1016/S1053-8119(03)00073-9

Delgado, M. R., Nearing, K. I., Ledoux, J. E., \& Phelps, E. a. (2008). Neural circuitry underlying the regulation of conditioned fear and its relation to extinction. Neuron, 59(5), 829-38. doi:10.1016/j.neuron.2008.06.029

Domsch, S., Linke, J., Heiler, P. M., Kroll, A., Flor, H., Wessa, M., \& Schad, L. R. (2013). Increased BOLD sensitivity in the orbitofrontal cortex using slicedependent echo times at 3 T. Magnetic Resonance Imaging, 31(2), 201-11. doi:10.1016/j.mri.2012.06.020

Etkin, A., \& Wager, T. D. (2007). Functional neuroimaging of anxiety: a meta-analysis of emotional processing in PTSD, social anxiety disorder, and specific phobia. The American Journal of Psychiatry, 164(10), 1476-1488. doi:10.1176/appi.ajp.2007.07030504

Farmer, A. S., \& Kashdan, T. B. (2012). Social anxiety and emotion regulation in daily life: spillover effects on positive and negative social events. Cognitive Behaviour Therapy, 41(2), 152-62. doi:10.1080/16506073.2012.666561 
Forman, S. D., Cohen, J. D., Fitzgerald, M., Eddy, W. F., Mintun, M. a, \& Noll, D. C. (1995). Improved assessment of significant activation in functional magnetic resonance imaging (fMRI): use of a cluster-size threshold. Magnetic Resonance in Medicine, 33(5), 636-47.

Goebel, R., Esposito, F., \& Formisano, E. (2006). Analysis of functional image analysis contest (FIAC) data with brainvoyager QX: From single-subject to cortically aligned group general linear model analysis and self-organizing group independent component analysis. Human Brain Mapping, 27(5), 392-401. doi:10.1002/hbm.20249

Goebel, R., Zilverstand, A., \& Sorger, B. (2010). Real-time fMRI-based brain-computer interfacing for neurofeedback therapy and compensation of lost motor functions. Imaging in Medicine, 2(4), 407-415. doi:10.2217/iim.10.35

Goldin, P. R., Manber-Ball, T., Werner, K., Heimberg, R., \& Gross, J. J. (2009). Neural mechanisms of cognitive reappraisal of negative self-beliefs in social anxiety disorder. Biological Psychiatry, 66(12), 1091-9. doi:10.1016/j.biopsych.2009.07.014

Gross, J. J. (2002). Emotion regulation: affective, cognitive, and social consequences. Psychophysiology, 39(3), 281-91. doi:10.1017.S0048577201393198

Gross, J. J., \& John, O. P. (2003). Individual differences in two emotion regulation processes: Implications for affect, relationships, and well-being. Journal of Personality and Social Psychology, 85(2), 348-362. doi:10.1037/00223514.85.2.348

Haller, S., Birbaumer, N., \& Veit, R. (2010). Real-time fMRI feedback training may improve chronic tinnitus. European Radiology, 20(3), 696-703. doi:10.1007/s00330-009-1595-z

Hauner, K. K., Mineka, S., Voss, J. L., \& Paller, K. a. (2012). Exposure therapy triggers lasting reorganization of neural fear processing. Proceedings of the National Academy of Sciences of the United States of America, 109(23), 9203-8. doi:10.1073/pnas.1205242109

Hutton, C., Josephs, O., Stadler, J., Featherstone, E., Reid, a, Speck, O., ... Weiskopf, N. (2011). The impact of physiological noise correction on fMRI at $7 \mathrm{~T}$. Neurolmage, 57(1), 101-12. doi:10.1016/j.neuroimage.2011.04.018

Jo, H. J., Saad, Z. S., Simmons, W. K., Milbury, L. a, \& Cox, R. W. (2010). Mapping sources of correlation in resting state FMRI, with artifact detection and removal. Neurolmage, 52(2), 571-82. doi:10.1016/j.neuroimage.2010.04.246

Johnson, K. a, Hartwell, K., LeMatty, T., Borckardt, J., Morgan, P. S., Govindarajan, K., ... George, M. S. (2012). Intermittent "real-time" fMRI feedback is superior to continuous presentation for a motor imagery task: a pilot study. Journal of Neuroimaging: Official Journal of the American Society of Neuroimaging, 22(1), 58-66. doi:10.1111/j.1552-6569.2010.00529.x

Kamphuis, J. H., \& Telch, M. J. (2000). Effects of distraction and guided threat reappraisal on fear reduction during exposure-based treatments for specific fears. Behaviour Research and Therapy, 38(12), 1163-1181. 
Kessler, R. C., Aguilar-Gaxiola, S., Alonso, J., Chatterji, S., Lee, S., Ormel, J., ... Wang, P. S. (2011). The Global Burden of Mental Disorders: An Update from the WHO. Epidemiologia E Psichiatria Sociale, 18(1), 23-33.

Kessler, R. C., Petukhova, M., Sampson, N. A., Zaslavsky, A. M., \& Wittchen, H.-U. (2012). Twelve-month and lifetime prevalence and lifetime morbid risk of anxiety and mood disorders in the United States. International Journal of Methods in Psychiatric Research, 21(3), 169-84. doi:10.1002/mpr.1359

Klorman, R., Weerts, T. C., Hastings, J. E., Melamed, B. G., \& Lang, P. J. (1974). Psychometric description of some specific-fear questionnaires. Behavior Therapy, 5(3), 401-409.

Linden, D. E. J., Habes, I., Johnston, S. J., Linden, S., Tatineni, R., Subramanian, L., ... Goebel, R. (2012). Real-time self-regulation of emotion networks in patients with depression. PloS One, 7(6), e38115. doi:10.1371/journal.pone.0038115

Morawetz, C., Holz, P., Lange, C., Baudewig, J., Weniger, G., Irle, E., \& Dechent, P. (2008). Improved functional mapping of the human amygdala using a standard functional magnetic resonance imaging sequence with simple modifications. Magnetic Resonance Imaging, 26(1), 45-53. doi:10.1016/j.mri.2007.04.014

Muris, P., \& Merckelbach, H. (1996). A comparison of two spider fear questionnaires. Journal of Behavior Therapy and Experimental Psychiatry, 27(96), 241-244.

New, A. S., Fan, J., Murrough, J. W., Liu, X., Liebman, R. E., Guise, K. G., ... Charney, D. S. (2009). A functional magnetic resonance imaging study of deliberate emotion regulation in resilience and posttraumatic stress disorder. Biological Psychiatry, 66(7), 656-64. doi:10.1016/j.biopsych.2009.05.020

Ochsner, K. N., Silvers, J. a, \& Buhle, J. T. (2012). Functional imaging studies of emotion regulation: a synthetic review and evolving model of the cognitive control of emotion. Annals of the New York Academy of Sciences, 1251, E1-24. doi:10.1111/j.1749-6632.2012.06751.x

Ost, L.-G. (2008). Cognitive behavior therapy for anxiety disorders: 40 years of progress. Nordic Journal of Psychiatry, 62(1), 5-10. doi:10.1080/08039480802315590

Prigatano, G. P., \& Johnson, H. J. (1974). Autonomic nervous system changes associated with a spider phobic reaction. Journal of Abnormal Psychology, 83(2), 169-177. doi:10.1037/h0036476

Pull, C. B. (2008). Recent trends in the study of specific phobias. Current Opinion in Psychiatry, 21(1), 43-50. doi:10.1097/YCO.0b013e3282f30086

Rheinberg, F., Vollmeyer, R., \& Burns, B. D. (2001). QCM : A questionnaire to assess current motivation in learning situations. Diagnostica, 47(2), 57-66. doi:10.1026//0012-1924.47.2.57

Robinson, S., Windischberger, C., Rauscher, a, \& Moser, E. (2004). Optimized 3 T EPI of the amygdalae. Neurolmage, 22(1), 203-10. doi:10.1016/j.neuroimage.2003.12.048

Sarlo, M., Palomba, D., Angrilli, A., \& Stegagno, L. (2002). Blood phobia and spider phobia: Two specific phobias with different autonomic cardiac modulations. Biological Psychology, 60(2-3), 91-108. 
Scheinost, D., Stoica, T., Saksa, J., Papademetris, X., Constable, R. T., Pittenger, C., \& Hampson, M. (2013). Orbitofrontal cortex neurofeedback produces lasting changes in contamination anxiety and resting-state connectivity. Translational Psychiatry, 3(4), e250. doi:10.1038/tp.2013.24

Schienle, A., Schäfer, A., Hermann, A., Rohrmann, S., \& Vaitl, D. (2007). Symptom provocation and reduction in patients suffering from spider phobia: an fMRI study on exposure therapy. European Archives of Psychiatry and Clinical Neuroscience, 257(8), 486-93. doi:10.1007/s00406-007-0754-y

Scott, N. W., McPherson, G. C., Ramsay, C. R., \& Campbell, M. K. (2002). The method of minimization for allocation to clinical trials. a review. Controlled Clinical Trials, 23(6), 662-74.

Sehlmeyer, C., Schöning, S., Zwitserlood, P., Pfleiderer, B., Kircher, T., Arolt, V., \& Konrad, C. (2009). Human fear conditioning and extinction in neuroimaging: a systematic review. PloS One, 4(6), e5865. doi:10.1371/journal.pone.0005865

Sheehan, D. V, Lecrubier, Y., Sheehan, K. H., Amorim, P., Janavs, J., Weiller, E., ... Dunbar, G. C. (1998). The Mini-International Neuropsychiatric Interview (M.I.N.I.): the development and validation of a structured diagnostic psychiatric interview for DSM-IV and ICD-10. The Journal of Cinical Psychiatry, 59 Suppl 2, 22-33;quiz 34-57. doi:10.1590/S1516-44462000000300003

Sloan, T., \& Telch, M. J. (2002). The effects of safety-seeking behavior and guided threat reappraisal on fear reduction during exposure: An experimental investigation. Behaviour Research and Therapy, 40(3), 235-251.

Somerville, L. H., Wagner, D. D., Wig, G. S., Moran, J. M., Whalen, P. J., \& Kelley, W. M. (2013). Interactions between transient and sustained neural signals support the generation and regulation of anxious emotion. Cerebral Cortex (New York, N.Y. : 1991), 23(1), 49-60. doi:10.1093/cercor/bhr373

Subramanian, L., Hindle, J. V, Johnston, S., Roberts, M. V, Husain, M., Goebel, R., \& Linden, D. (2011). Real-time functional magnetic resonance imaging neurofeedback for treatment of Parkinson's disease. The Journal of Neuroscience: The Official Journal of the Society for Neuroscience, 31(45), 16309-17. doi:10.1523/JNEUROSCI.3498-11.2011

Szymanski, J. (1995). Fear of spiders questionnaire. Journal of Behavior Therapy and Experimental Psychiatry, 26(1), 31-34.

Talairach, J., \& Tournoux, P. (1988). Co-planar stereotaxic atlas of the human brain: 3-dimensional proportional system: an approach to cerebral imaging. Neuropsychologia (Vol. 39). New York: Thieme Medical Publishers.

Weiskopf, N. (2012). Real-time fMRI and its application to neurofeedback. Neurolmage, 62(2), 682-92. doi:10.1016/j.neuroimage.2011.10.009

Weiskopf, N., Hutton, C., Josephs, O., Turner, R., \& Deichmann, R. (2007). Optimized EPI for fMRI studies of the orbitofrontal cortex: compensation of susceptibilityinduced gradients in the readout direction. MAGMA, 20(1), 39-49. doi:10.1007/s10334-006-0067-6

Weissenbacher, A., Kasess, C., Gerstl, F., Lanzenberger, R., Moser, E., \& Windischberger, C. (2009). Correlations and anticorrelations in resting-state 
functional connectivity MRI: A quantitative comparison of preprocessing strategies. Neurolmage, 47(4), 1408-1416.

Wittchen, H.-U., \& Jacobi, F. (2005). Size and burden of mental disorders in Europe-a critical review and appraisal of 27 studies. European Neuropsychopharmacology: The Journal of the European College of Neuropsychopharmacology, 15(4), 357-76. doi:10.1016/j.euroneuro.2005.04.012

Young, K. D., Zotev, V., Phillips, R., Misaki, M., Yuan, H., Drevets, W. C., \& Bodurka, J. (2014). Real-time FMRI neurofeedback training of amygdala activity in patients with major depressive disorder. PloS One, 9(2), e88785. doi:10.1371/journal.pone. 0088785 


\section{5}

\section{fMRI neurofeedback in ADHD patients:}

"fMRI-based neurofeedback from anterior cingulate cortex for adults with Attention-Deficit/Hyperactivity Disorder. A proof of concept study."

Based on: Zilverstand, A., Sorger, B., Slaats, D., Kan, C., Goebel, R., \& Buitelaar, J. "fMRl-based neurofeedback from anterior cingulate cortex for adults with Attention-Deficit/Hyperactivity Disorder. A proof of concept study." [in preparation]. 


\section{Abstract}

Attention-deficit/hyperactivity disorder (ADHD) is associated with poor cognitive control and hypofunctioning of the dorsal anterior cingulate cortex (ACC) during continuous performance, response inhibition and switching tasks. We investigated for the first time whether fMRI neurofeedback training targeted at increasing activation levels in the ACC in adults with ADHD leads to reduction of ADHD symptoms and improved cognitive functioning. An exploratory, randomized controlled treatment study with blinding of the participants was conducted. Participants with ADHD ( $n=7$ in the active feedback condition, and $n=3$ in the control condition) attended four weekly training sessions, during which they performed a mental calculation task at varying levels of difficulty, in order to learn how to up-regulate ACC activation. Prior, and after the training ADHD symptoms and cognitive functioning were assessed by neuropsychological testing. Results showed that neurofeedback participants achieved a significant increase in ACC activation levels over sessions. The neurofeedback group did not show a significant reduction of ADHD symptoms, but improved considerably and significantly on measures of attentional control and working memory. Individual modulation performance was highly correlated with the ability to sustain attention and working memory capacity, but modulation success was not highly correlated with treatment change. The contribution of the provided neurofeedback information to treatment change can therefore not be finally evaluated. 


\section{Introduction}

Attention-deficit/hyperactivity disorder (ADHD) is a rather common childhood-onset neuropsychiatric disorder characterized by a pervasive pattern of inattention, and/or hyperactivity and impulsivity (American Psychiatric Association, 2000, 2013). The disorder persists into adulthood in one third of the cases or more, with prevalence in adults being estimated at 2-4\% (Kessler et al., 2006; Kooij et al., 2005; Simon, Czobor, Bálint, Mészáros, \& Bitter, 2009). The first-line treatment of moderate to severe ADHD in children and adolescents is prescription of medication, mostly psychostimulants. This is associated with response rates of $70 \%$ or higher, a reduction of ADHD symptoms, and improved performance on sustained attention and working memory tests (Coghill, Rhodes, \& Matthews, 2007; Pietrzak, Mollica, Maruff, \& Snyder, 2006; Swanson, Baler, \& Volkow, 2011). However, evidence for long-term efficacy of medication is inconsistent (Van de Loo-Neus, Rommelse, \& Buitelaar, 2011), and there are concerns about potential side-effects of long-term use of medication (Graham et al., 2011). Medication treatment of ADHD in adults has proven to be less effective, with response rates of $50 \%$ to psychostimulants and as low as $20 \%$ when non-stimulants were taken (Faraone \& Glatt, 2010).

Several non-pharmacological treatments have been developed and evaluated for treatment efficacy in ADHD, among which electroencephalography (EEG) neurofeedback. The idea of neurofeedback treatment is that participants target aspects of neuronal functioning directly by learning self-control through feedback, mostly based on the theta/beta frequency ratio. A systematic and comprehensive meta-analysis of non-pharmacological treatments for ADHD documented significant treatment effects for EEG-neurofeedback (Sonuga-Barke et al., 2013). However, when the assessment of outcome was based on blinded raters, treatment effects were substantially attenuated to non-significant levels (Sonuga-Barke et al., 2013). In line with the metaanalysis, a recent double-blind randomized placebo-controlled EEG-neurofeedback study in children and adolescents with ADHD was unable to establish treatment effects on clinical symptoms and neurocognitive performance (van Dongen-Boomsma, Vollebregt, Slaats-Willemse, \& Buitelaar, 2013; Vollebregt, van Dongen-Boomsma, Buitelaar, \& Slaats-Willemse, 2013). This has spurred interest into the development of other neurofeedback methods, as for example neurofeedback based on functional magnetic resonance imaging (fMRI). 
The advantage of functional MRI (fMRI)-neurofeedback over EEGneurofeedback is a higher spatial resolution and full brain coverage, and therefore also a possibly more specific treatment response. Since the mid-1990s several research groups have been working on the development of fMRI real-time techniques that allow for immediate processing and analysis of data during fMRI scanning. Current real-time fMRI procedures include most state-of-the-art data preprocessing and analysis steps of its classical offline counterpart (Weiskopf, 2012). Numerous studies have shown that participants are indeed able to control their brain activation patterns in very specific ways (Goebel, Zilverstand, \& Sorger, 2010; Weiskopf, 2012). Importantly, specific behavioral effects were correlated with specific changes in brain activation patterns, such as up-regulation of motor areas improved motor skills, or up-regulation of regions in the speech network lead to improved accuracy in a language task (Goebel et al., 2010). First clinical trials indicated that patients with disorders as diverse as chronic pain, tinnitus, Parkinson disease, mood and anxiety disorders may experience relief from their symptoms after a fMRI-neurofeedback training (DeCharms et al., 2005; Haller, Birbaumer, \& Veit, 2010; Linden et al., 2012; Scheinost et al., 2013; Subramanian et al., 2011; Young et al., 2014) (previous chapter). However, the efficacy of fMRI neurofeedback training in ADHD has not been investigated so far.

The general approach pursued in neurofeedback treatment is using feedback as a tool to guide self-regulation, aiming at normalization of deviant brain activation patterns. One of the most consistently found abnormalities in ADHD is weak cognitive control, and associated deviant functioning of frontal, cingulate and parietal cortical brain regions (Bush, 2011). The brain region that has been most consistently linked to cognitive control and the core ADHD symptoms is the dorsal anterior cingulate cortex (ACC) (Bush, 2011). fMRI research has consistently found a characteristic pattern of cingulate hypo-activation in subjects with ADHD, compared to non-ADHD subjects, while performing tasks that require effortful control, e.g. interference task, continuous performance test, switch task, and response inhibition task (Bush, 2011; Bush et al., 1999, 2008; Cubillo et al., 2010; Dickstein, Bannon, Castellanos, \& Milham, 2006; Schneider et al., 2010). Moreover, hypo-activation of the ACC was found to normalize after successful treatment with ADHD medication (Bush et al., 2008). Thus, normalization of ACC activity seems to be a crucial component of a successful treatment. In the current fMRI neurofeedback study we therefore aimed at training subjects with ADHD to voluntarily up-regulate activation levels in the dorsal ACC. 
An exploratory randomized controlled treatment study with blinding of the participants was designed to investigate if up-regulation of the dorsal ACC could be achieved through self-modulation guided by $\mathrm{FMRI}$ neurofeedback, and if fMRI neurofeedback training was successful in reducing ADHD symptoms and improving cognitive functioning. Participants attended four weekly cognitive training sessions guided by fMRI neurofeedback. ADHD symptoms and cognitive functioning were assessed a week prior, and a week after training. Participants in the control group underwent the same procedure, but were not provided with neurofeedback information.

\section{Methods}

\section{Participants}

Participants were recruited among referrals to the Department of Psychiatry of the Radboud University Medical Center, Nijmegen, The Netherlands. Participants were included if: they received a diagnosis of ADHD according to the DSM-IV TR criteria (American Psychiatric Association, 2000); were older than 18 years; psychopharmacanaïve or -free, or being on a fixed dose of psychostimulant medication for the study period; passed fMRI screening criteria; and had an IQ $>90$ according to block design and vocabulary test of the Wechsler Adult Intelligence Scale [WAIS-IV-NL, (Cotan, 2012; Wechsler, 2008)]. The administered short version (Vocabulary, Block design) was selected due to its high validity coefficient $(0.85)$ relative to the full intelligence test (Sattler, 2001). Participants were excluded if: they participated in another clinical trial simultaneously; participated previously in neurofeedback training; had another significant medical condition or regular use of medication other than psychostimulants; current diagnosis of one or more Axis-I diagnosis other than ADHD according to the DSM-IV TR criteria (American Psychiatric Association, 2000) (e.g. depression, psychosis, tics, autism, eating disorder); current alcohol or drug abuse according to the DSM-IV TR criteria (American Psychiatric Association, 2000).

The presence of ADHD symptoms in childhood and adulthood (i.e., current) was assessed using a Semi-Structured Interview for ADHD (Kooij, 2003) (see http://www.divacenter.eu/). This interview has been used in previous studies of adult ADHD and shown to be both reliable and valid (Bron et al., 2014; Kooij, 2003; Kooij et al., 2005; Van Veen, Kooij, Boonstra, Gordijn, \& Van Someren, 2010). Confirmation of 


\begin{tabular}{l|l|l|}
\hline \multicolumn{3}{l}{ Table 1. Characteristics of study participants } \\
\hline $\begin{array}{l}\text { Variables (Mean +l- SD) } \\
\text { (score pre-testing) }\end{array}$ & Control group & $\begin{array}{l}\text { Neurofeedback } \\
\text { group }\end{array}$ \\
\hline Gender (male/ female) & 1 male/ 2 female & 3 male/ 4 female \\
\hline Age & $37.3(14.2)$ & $34.0(11.0)$ \\
\hline IQ (WAIS VCl BD) & $102(7)$ & $110(9)$ \\
\hline ADHD medication (yes/ no) & 1 yes/ 2 no & 3 yes/ 4 no \\
\hline ADHD attention & $7.7(1.2)$ & $7.0(1.2)$ \\
\hline ADHD impulsivity/hyperactivity & $7.0(3.5)$ & $6.4(2.2)$ \\
\hline
\end{tabular}

Participants were ten adults, diagnosed with ADHD according to DSM-IV TR criteria. During the study period all participants were either on a stable dose or free of medication for ADHD symptoms (control group: one participant: $100 \mathrm{mg} /$ daily of atomoxetine hydrochloride (Strattera); neurofeedback group: one participant: $30 \mathrm{mg} /$ daily dexamfetamine, one participant: $15 \mathrm{mg} /$ daily dexamfetamine, one participant: $72 \mathrm{mg} /$ daily methylfenidaathydrochloride (Concerta)). Participants were included based on the ADHD diagnosis, medical screening, and estimated IQ score $(I Q>90)$. They were randomly assigned to a group using a minimization procedure, which balanced IQ score, ADHD medication use, and ADHD symptoms for inattention, and hyperactivity/impulsivity.

the developmental history and childhood occurrence of ADHD symptoms was obtained from the parents or, when unavailable, an older sibling of the patient. In addition, the Dutch versions of the ADHD-DSM-IV Rating Scales (DuPaul, Power, Anastopoulus, \& Reid, 1998) were completed by patient, spouse, parent, and investigator to gather information on the exact DSM-IV criteria for ADHD in childhood and adulthood. The following was required to be the case for assignment of a full diagnosis of adult ADHD: (1) at least 6 of the 9 DSM-IV criteria for inattention and/or hyperactivity/impulsivity had to be met for diagnosis of childhood ADHD and at least 5 of the 9 criteria for diagnosis of adult ADHD; (2) a chronic course of persistent ADHD symptoms from childhood to adulthood had to be reported; and (3) a moderate to severe level of impairment that can be attributed to the symptoms of ADHD had to be experienced. The cut-off point of 5 out of the 9 criteria for diagnosis of adult ADHD is based upon the literature and epidemiological data using the same DSM-IV ADHD Rating Scale (Kooij et al., 2005; Murphy \& Barkley, 1996) and consistent with the DSM-5 algorithm for ADHD.

Fifteen participants volunteered and were screened for inclusion and exclusion criteria. Five participants had to be excluded because they did not fulfill the IQ criterion. Ten participants were enrolled in the study (table 1). Participants were randomly 
assigned to a group using a minimization procedure (sequential balancing) with the factors IQ score, psychostimulant medication, and the DSM-IV rating scale scores for ADHD symptoms (Kooij et al., 2005). This restricted randomization procedure has been shown to be efficient in balancing several factors in studies with a small sample size (Borm, Hoogendoorn, den Heijer, \& Zielhuis, 2005; Scott, McPherson, Ramsay, \& Campbell, 2002). All participants received a small financial compensation ( $8 € /$ hour), and gave their written informed consent prior to the experiment that was conducted in conformity with the Declaration of Helsinki, and approved by the local Medical Ethics Committee.

\section{General Procedure}

After enrollment, participants attended six sessions, including the behavioral preassessment, four weekly training sessions, and the behavioral post-assessment (figure 1). During the 90-minute pre-assessment they first completed the ADHD DSMIV rating scales (Kooij et al., 2005), were then familiarized with the interference task employed in the MRI session (Bush \& Shin, 2006), then completed four neuropsychological tests, and finally the intelligence test (Wechsler, 2008). During the post-assessment they completed the same tests in the same order, except for the intelligence test. During the first MRI session, participants were informed that the goal of the study was to investigate if up-regulation of dorsal ACC through a mental task would have a positive impact on ADHD symptoms. Only the neurofeedback group was informed that they were participating in a neurofeedback study. Participants were then instructed on the general procedure, and filled in a Questionnaire of Current Motivation [QCM, (Rheinberg, Vollmeyer, \& Burns, 2001), in order to assess motivational state during each session. Each MRI session then consisted of a 7-minute anatomical scan, two 9-minute localization tasks, used to functionally define the target regions from which the neurofeedback would be derived in the neurofeedback group, three 8-minute functional training runs with feedback for the neurofeedback participants only, and a 8minute transfer run, during which both groups did not receive feedback.

\section{Localization of target regions}

The first localization task was the multi-source interference task [MSIT, (Bush \& Shin, 2006)], which has been specifically developed to functionally localize the prefrontal regions involved in cognitive control in ADHD on an individual level (Bush, Shin, 


\begin{tabular}{|c|c|c|c|}
\hline $\begin{array}{l}\text { screening } \\
30 \text { min }\end{array}$ & $\begin{array}{l}\text { pre-test } \\
90 \text { min }\end{array}$ & $\begin{array}{l}\mathbf{4 x} \text { training (weekly) } \\
90 \mathrm{~min}\end{array}$ & $\begin{array}{l}\text { post-test } \\
90 \mathrm{~min}\end{array}$ \\
\hline $\begin{array}{l}\text { Information } \\
\text { Screening } \\
\text { ADHD diagnosis } \\
\text { Health screening } \\
\text { fMRI screening }\end{array}$ & $\begin{array}{l}\text { NP-Tests } \\
\text { ADHD rating } \\
\text { MSIT intro } \\
\text { SA-DOTS pre } \\
\text { SART pre } \\
\text { N-back pre } \\
\text { WAIS WMI pre } \\
\text { WAIS VCI/ PRI }\end{array}$ & $\begin{array}{l}\text { Questionnaire } \\
\text { motivation QCM } \\
\text { fMRI } \\
\text { anatomy } \\
\text { Localizer MSIT } \\
\text { Localizer MCT } \\
\text { neurofeedback } \\
\text { Questionnaire } \\
\text { success + strategy } \\
\text { Questionnaire } \\
\text { motivation QCM } \\
\text { fMRI } \\
\text { anatomy } \\
\text { Localizer MSIT } \\
\text { Localizer MCT } \\
\text { control-training } \\
\text { Questionnaire } \\
\text { success + strategy }\end{array}$ & $\begin{array}{l}\text { NP-Tests } \\
\text { ADHD rating } \\
\text { MSIT post } \\
\text { SA-DOTS post } \\
\text { SART post } \\
\text { N-back post } \\
\text { WAIS WMI post } \\
\text { Disclosure }\end{array}$ \\
\hline
\end{tabular}

\section{Figure 1: Experimental design.}

Participants were first screened, and then attended six sessions. During behavioral pre- and post- testing they completed the ADHD rating scale (ADHD rating), were then familiarized with the multi-source interference task (MSIT) employed in the MRI session (MSIT intro), then completed four neuropsychological tests (SA-DOTS $=$ sustained attention dots task, SART = sustained attention response task, N-back = visuo-spatial 2-back working memory task, WAIS $\mathrm{WMI}=$ Wechsler Adult Intelligence Scale Working memory index), and last the intelligence test (WAIS VCI = Wechsler Adult Intelligence Scale Verbal Comprehension Index, WAIS PRI $=$ Wechsler Adult Intelligence Scale Processing Speed Index). During the fMRI session they received an instruction on all tasks they would perform in the scanner and then filled in the Questionnaire of Current Motivation (QCM). Each MRI session consisted of an anatomical scan, two localization tasks for defining the target regions, from which the feedback would be derived, and the training runs.

Holmes, Rosen, \& Vogt, 2003; Bush \& Shin, 2006). This task combines three different cognitive interference tasks: the Eriksen Flanker task, the Counting Stroop, and the Simon effect task (Bush et al., 2003). During each trial, participants were presented with a set of three numbers $(1,2,3$, or 0$)$, with one number (target) differing from the other two (matching distractors). Participants were instructed to report the value of the number which is different via a button press (Bush \& Shin, 2006). In control trials (100, 020 , or 003$)$ the target number $(1,2$, or 3$)$ matched its position, and the distractors 
were zeros. In interference trials, the target number did not match its position, and was accompanied by distractors that were themselves potential targets (e.g., 211, the correct answer is ' 2 '). Both sorts of trials were presented blocked, in series of $42 \mathrm{~s}$, with 24 trials of $1750 \mathrm{~ms}$. Four control and four interference blocks were presented in pseudo-randomized order, with intermittent rest periods of 18-24 s (total of 9 minutes). The dorsal ACC was defined functionally by contrasting activation during interference blocks (interference) with resting periods (rest) during online analysis, and interference blocks (interference) with control blocks (control) during the post-hoc whole-brain analysis conducted to verify the online procedure.

As a second localization task, participants were instructed to perform a mediumdifficulty mental calculation task, similar to the mental task they would perform during later training runs. They were asked to start with the number 100 , subtract a single digit number in their head, and keep subtracting this number from the result. To ensure that the task was of medium difficulty, the number was selected individually, and participants were asked to practice out aloud prior to scanning. As a control task, participants chose a song verse, which was well-known to them, and they could sing effortlessly in their head. The localization procedure included five $26 \mathrm{~s}$ blocks of mental calculation, and five $26 \mathrm{~s}$ blocks mental singing, visually cued, and presented in alternating order with intermittent-resting periods of $26 \mathrm{~s}$ (total of 9 minutes). Pilot measurements had showed that the same ACC region as with the MSIT task could be localized, either by contrasting mental calculation (mental calculation) versus resting (rest), or versus mental singing (mental singing). Two different localizer tasks were employed to assure the definition of the target regions on an individual level in all sessions, to verify functional overlap between the MSIT and mental calculation task post-hoc, and as warm-up task prior to the neurofeedback training.

\section{MRI training runs}

Prior to scanning participants were instructed that the cognitive training task used to up-regulate dorsal ACC would consist of variants of the mental calculation task used during the localization procedure. They received a detailed explanation on how to generate different number series varying in levels of difficulty through changing three different task aspects: 1) tempo, 2) magnitude of the numbers, and 3) variations in the operation rule. The specific instructions were developed based on fMRI studies on 
activation levels during different arithmetic operations (Delazer et al., 2003; Fehr, Code, \& Herrmann, 2007). Participants were furthermore instructed to adapt the mental calculation task according to individual difficulty level throughout the experiment. They were explained that in order to get a sense of the level of difficulty, they would be asked to perform it at two different levels of difficulty, cued by a red box in the visual display (figure 2). When the visual display did not contain a red box, the instruction was to rest, when the red box appeared at a medium height they should select a mental calculation task of medium difficulty ( $50 \%$ difficulty), and when the red box was displayed at the top, they should perform a mental calculation series of high difficulty (100\% difficulty). Participants were told that they would be reminded of all possible task variations at the beginning of each run. Each cognitive training run consisted of four $30 \mathrm{~s}$ blocks of each condition (50\% difficulty, $100 \%$ difficulty) in pseudo-randomized order, with intermittent 20 s rest periods (total duration 8 minutes). All participants performed four cognitive training runs per MRI session. Neurofeedback participants received neurofeedback during the first three training runs of each session, and did not receive any feedback during the last run, in order to test their ability to transfer gained knowledge to a situation without feedback.

After the general instruction, the neurofeedback group was instructed that the current individual activation level in the dorsal anterior cingulate would be visualized by filled grey squares in the thermometer display (figure 2). They were explained that the neurofeedback would indicate how difficult the task was at this point of time, and that they should adapt the mental calculation task according to the feedback. Additionally, they received an instruction on noise levels, and the delay of the bloodoxygen-level dependent (BOLD) signal. They were shown data from a previous test participant, and given the chance to experience the length of the BOLD delay through a small simulation task. Finally, participants from both groups were instructed that it was important to perform the task systematically, and continuously, to remain calm during resting periods, and move as little as possible. Also, all participants, including the control participants, were aware that the data would be analyzed online.

\section{MRI imaging parameters}

The images were acquired at the Donders Centre for Cognitive Neuroimaging, Radboud University Nijmegen, on a 3T scanner (Tim Trio, Siemens Healthcare, Germany), equipped with a 32 channel head coil. Functional images were acquired 
with a repeated single-shot echo-planar imaging (EPI) sequence with TE $=30 \mathrm{~ms}$, $\mathrm{TR}=2000 \mathrm{~ms}, \mathrm{FA}=80^{\circ}, \mathrm{FOV}=192 \times 192 \mathrm{~mm}^{2}$, matrix $=64 \times 64$, voxel size $3 \times 3 \times 3 \mathrm{~mm}^{3}$, and bandwidth $=1628 \mathrm{~Hz} / \mathrm{Px}$, scanning 35 slices per volume, covering the whole brain. Anatomical images were collected with a 3D MPRAGE sequence: TR $=2300 \mathrm{~ms}$, TE $=3.92 \mathrm{~ms}, \mathrm{FOV}=256 \times 256 \mathrm{~mm}^{2}$, voxel size $1 \times 1 \times 1 \mathrm{~mm}^{3}, 192$ slices, with duration 7 minutes.

A

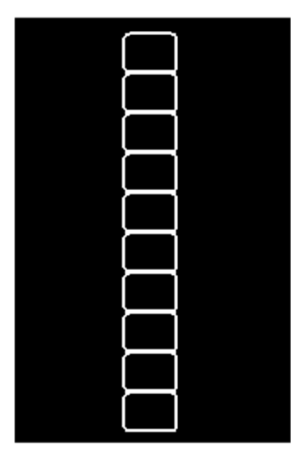

B

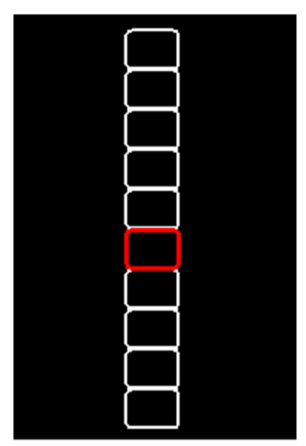

C

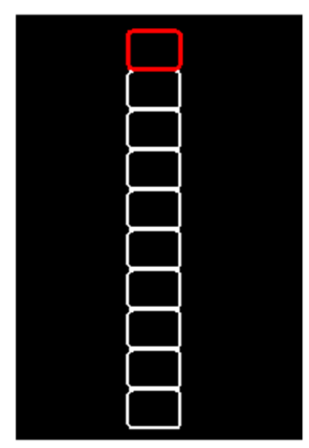

D
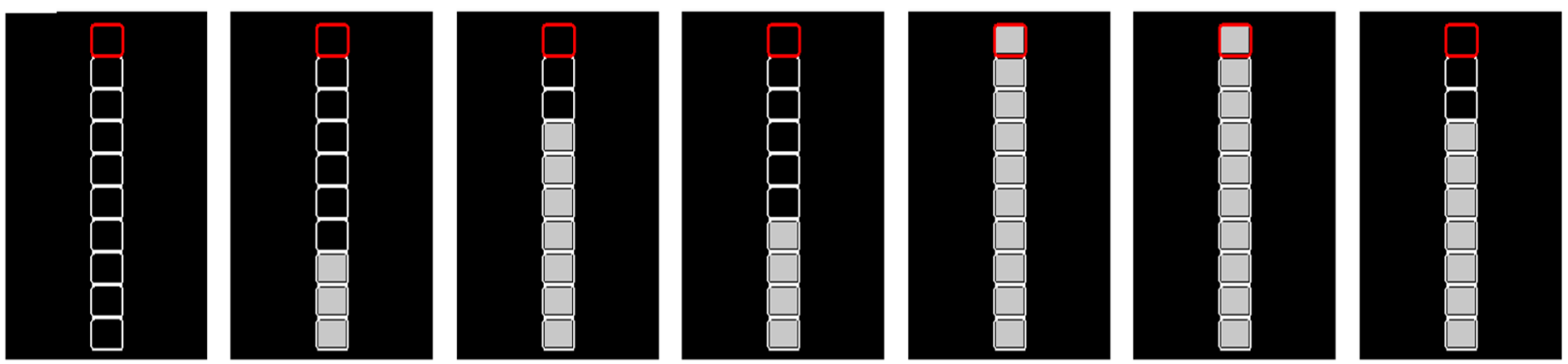

time

\section{Figure 2: Visual display during cognitive training runs.}

Participants were asked to perform a mental calculation task at varying levels of difficulty. When the visual display did not contain a red box, they were instructed to rest $(\mathbf{A})$, when the red box was at medium height, they should select a mental calculation task of medium difficulty (50\% difficult, B), and when the red box was displayed at the top, they should perform a mental calculation series of high difficulty $(100 \%$ difficult, C). The neurofeedback group was furthermore explained that the feedback on their individual current activation level in anterior cingulate cortex would be visualized by filled grey squares in the thermometer display (D). Before scanning, neurofeedback participants were shown data from a test participant, and had the chance to experience the length of the BOLD delay through a simulation task. 


\section{Real-time MRI data analysis}

Anatomical images were processed using BrainVoyager QX (BVQX, Version 2.7, Brain Innovation B.V., Maastricht, The Netherlands), and loaded into the real-time data analysis software TurboBrainVoyager (TBV, Version 3.2 beta, Brain Innovation B.V., Maastricht, The Netherlands) prior to the start of functional scanning. Analysis of all functional data was performed in TBV. After discarding the first four volumes of each functional run, the data was automatically aligned to the anatomical scan, and preprocessed in real-time using intra-session 3D rigid-body motion correction, and linear drift confound predictors. An online voxel-wise general linear model (GLM) was computed, convolving the task predictors with a standard two-gamma hemodynamic response function. Prior to the definition of the target regions, the data from the localization run was high-pass filtered with a GLM Fourier basis set (3 cycles), and thresholded at $\mathrm{t}=3$, with an additional cluster threshold of four significant voxels. In general, the target regions within the dorsal anterior cingulate (ACC target regions) were defined based on the first localization task (MSIT), contrasting interference with rest. Pilot measurements had shown that this contrast was more robust than interference versus control, and localized the same network. If no significant cluster within the anterior cingulate cortex was found during the first localization task $(\sim 10 \%$ sessions), the target regions were defined based on the data of the second localization task, contrasting mental calculation with rest. Generally, the most anterior ACC cluster was selected, as the anterior ACC showed the strongest under-activation in ADHD patients (Bush et al., 2008). After each imaging volume TBV calculated the average percent signal change (PSC) in the target region relative to a $14 \mathrm{~s}$ baseline from the previous rest period, and updated the thermometer display. The feedback calculations were generally performed in less than one TR $(2 s)$, while there was an additional delay of one TR (2s) due to technical problems for the first three neurofeedback participants. The maximum PSC of the thermometer was adjusted each session to be $150 \%$ of the average activation level in the target region during the mental calculation condition of the second localization task (if $<0.3 \%$ PSC, the activation level during the interference condition of the MSIT was used as a reference). The maximal PSC displayed in the neurofeedback group increased slightly over sessions (session 1: $0.81 \%$, session 2: $0.86 \%$, session $3: 0.90 \%$, session $4: 0.95 \%$ ). 


\section{Post-hoc MRI data analysis}

Functional and anatomical images were pre-processed in BrainVoyager QX, using the same parameters as during real-time analysis. Functional runs during which participants moved more than $5.00 \mathrm{~mm} /$ degree in any direction/rotation were excluded from post-hoc analysis. This applied to three functional runs from one neurofeedback participant (all from session 4: last three runs), and four functional runs from one control participant (session 2: first two training runs, session 3: last training run, session 4: second training run). The data from session 4 of another participant of the neurofeedback group was not obtained, as this participant did not participate in the last MRI session due to problems with scheduling. All remaining anatomical and functional data was spatially normalized to Talairach space to enable comparison between participants (Talairach \& Tournoux, 1988). A whole-brain random-effects GLM analysis was conducted to investigate if the targeted attentional control network was localized, and overlapped spatially with the defined ACC target regions. For this analysis wholebrain maps were thresholded using an initial voxel-threshold of $\alpha=0.05$ (Forman et al., 1995), and correcting for multiple comparisons using cluster-size thresholding with a cluster-level false positive rate of $\alpha=0.05$ (Forman et al., 1995; Goebel, Esposito, \& Formisano, 2006).

To evaluate the performance of the participants during localization and cognitive training runs, a post-hoc region-of-interest analysis of the BOLD response in the ACC target regions was performed. The estimated beta weights of the average BOLD response were extracted, and analyzed in SPSS Statistics (IBM SPSS Statistics 21; IBM Corporation, Armonk, NY, USA). They were submitted to within-group statistical analysis using repeated measures GLM with linear contrasts, modeling the factors task (interference, control; mental calculation, mental singing; 50\% difficulty, 100\% difficulty), and time (run, session). As measurements were still ongoing, betweengroup statistical comparisons were not performed due to the unbalanced, and small sample size. Effect sizes were estimated using partial eta squared (Cohen, 1973).

To evaluate moderating factors of individual modulation performance during cognitive training runs, three different performance indices were calculated based on the extracted beta weights from the ACC target regions (figure 3). First, an index of general task performance, which summarized mean activation levels across all four sessions. Second, an index of improvement over sessions, by calculating the increase in activation level between consecutive sessions, independent of task condition. Third, 
A

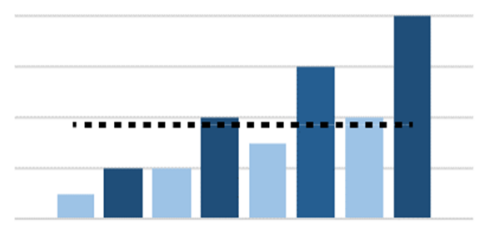

Training runs session 1-4
B

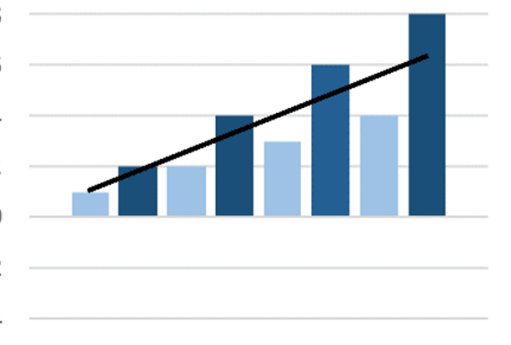

Training runs session 1-4
C

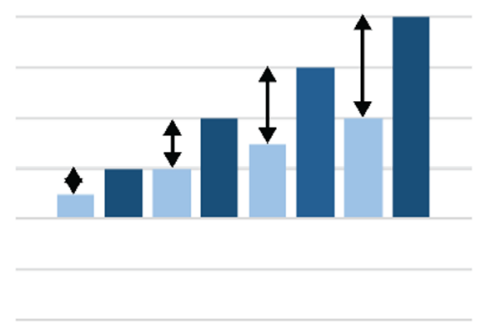

Training runs session 1-4

$50 \%$ difficulty $\quad 100 \%$ difficulty

Figure 3. Indices for quantifying individual modulation performance.

To evaluate individual performance during cognitive training runs, three different indices of individual modulation performance were calculated based on the individually estimated activation level in the ACC target region from each session. First, an index of general task performance was calculated by estimating the mean activation level across all four sessions (A). Second, an index of improvement over sessions was estimated, by calculating the increase in activation level between consecutive sessions, independent of condition (B). Third, the improvement in modulation was assessed, by computing the increase in differential activation between task conditions (50\% difficulty, $100 \%$ difficulty) over consecutive sessions (C).

an index of improvement in modulation, by computing the increase in differential activation between task conditions (50\% difficulty, 100\% difficulty) over consecutive sessions. Correlations between these indices and predictors from behavioral pretesting were computed.

\section{Pre-post behavioral assessment}

Participants with ADHD exhibit significant impairments with medium effect sizes on a range of executive functioning tasks, exhibiting the strongest and most consistent performance deviations in sustained attention tasks requiring response inhibition, and vigilance, as well as on working memory tasks, most often when spatial working memory is required (Nigg, 2005; Willcutt, Doyle, Nigg, Faraone, \& Pennington, 2005). Pre-post testing therefore assessed working memory in the spatial and verbal domain, and sustained attention through two different continuous performance tasks, which tapped two different aspects of sustained attention, being response inhibition, and vigilance. 
The first continuous performance tasks employed was the Sustained Attention Dots task [SA-DOTS, (de Sonneville, 1999)]. The SA-DOTS is a computerized visual sustained attention task, during which 50 series of 12 dot patterns were presented randomly, a total of 600 dot patterns. Each dot pattern consisted either of three, four, or five dots, and participants were required to press "yes" for four dots (target, $33 \%$ of trials), and "no" for three or five dots (non-targets, $67 \%$ of trials). The task was performed self-paced, with a post-response interval of $250 \mathrm{~ms}$, and took a total duration of 10-15 minutes, including 24 practice trials. Participants were instructed to perform it as fast, and accurately as possible. As the number of presented targets (33\%), and non-targets (67\%) was unequal, a response bias towards pressing "no" was invoked. Missed targets are therefore considered an index of response disinhibition, while false alarms are assumed to reflect vigilance (Marchetta, Hurks, De Sonneville, Krabbendam, \& Jolles, 2008; Slaats-Willemse, Swaab-Barneveld, De Sonneville, \& Buitelaar, 2005). The SA-DOTS has an excellent test-retest reliability (0.90-0.94), and provides performance z-scores in reference to a normed age sample (de Sonneville, 2005). It has also been shown to discriminate ADHD patients from healthy controls (Marchetta et al., 2008; Slaats-Willemse et al., 2005).

The second continuous performance task employed, was the Sustained Attention to Response Task [SART, (Robertson, Manly, Andrade, Baddeley, \& Yiend, 1997)], which is also sensitive to discriminating ADHD patients from healthy controls (O'Connell et al., 2008; Smilek, Carriere, \& Cheyne, 2010). The task consisted of 12 series with 18 trials, a total of 216 trials. During each trial a single digit (1-9) was presented for $900 \mathrm{~ms}$ in random order (each digit twice per series), with an interstimulus interval of $250 \mathrm{~ms}$. The total task duration was 6 minutes, including 36 practice trials. Participants were instructed to perform the task as fast and accurately as possible, and press a button after each digit, withholding their response only when a ' 3 ' was presented (non-target, $11 \%$ of trials). Due to the high number of targets $(89 \%$ of trials), a strong response bias towards pressing the button was induced, making this task especially sensitive for detecting impairments of response disinhibition, which are indexed by the number of false alarms.

The first working memory task used, was a 2-back visuo-spatial task, shown to be sensitive to ADHD impairments of working memory [2-back WM, (Hoogman, 2012)]. A series of white squares was presented, one at a time, each square being shown at a different of nine possible grid locations. Each square was presented for $500 \mathrm{~ms}$, 
followed by $800 \mathrm{~ms}$ fixation. In total, 10 series of 15 trials were presented in pseudorandom order, with 5 second breaks in-between series. The task had a total duration of 9 minutes, including the instruction, and 30 practice trials. Each series included 3-5 target trials, during which the white square appeared at the same location as two trials before (25\% targets). Participants were instructed to press the button as soon as a target appeared, and to perform the task as fast and accurately as possible. The main outcome measure for indexing working memory performance was the proportion of correct trials, calculated by subtracting the proportion of misses and false alarms from the total number of trials. All computerized tasks used during pre-post assessment and the MRI session were programmed and presented using Presentation software package (Version 16, Neurobehavioral Systems Inc., Albany, CA, USA).

To assess verbal working memory, two subtests from the standardized WAISIV-NL were selected (Digit Span, Letter-Number Sequencing). The frequently used Digit Span subtest has shown to be discriminative in detecting working memory impairments in ADHD (Willcutt et al., 2005), has a very good internal consistency $(>0.85)$, and an adequate test-retest reliability $(>0.75)$ (Iverson, 2001). For the Digit Span test, sequences of single digits with increasing length were presented verbally to the participant. The participant was instructed to repeat the sequences in the same order (forward), in reverse order (backward), or to sort the digits according to their value (order). Testing ceased as soon as the participant made two consecutive errors (duration $\sim 5$ minutes). For the Letter-Number Sequencing test, sequences of numbers and digits of increasing length were used, and the participant was asked to sort the digits according to value, and the letters alphabetically. The test was stopped after three consecutive errors (duration $\sim 5$ minutes). For both subtests age-referenced norm scores were calculated.

\section{Behavioral data analysis}

The scores from the questionnaires of current motivation were submitted to withingroup statistical analysis using repeated measures GLM with linear contrasts, modeling the factor time (session). All behavioral scores from pre-post assessment were analyzed for the within-group factor time (pre-post), to investigate if ADHD symptoms and cognitive functioning improved significantly. As measurements were still ongoing, between-group statistical comparisons were not performed due to the unbalanced, and small sample size. To evaluate if individual performance during the 
cognitive training runs was related to treatment change, the individual performance indices were correlated with treatment change. Finally, to investigate if improvement depended on impairment prior to the training, correlations between behavioral predictors from pre-testing, and positive treatment change were computed.

\section{Results}

\section{Localization of target regions}

Based on the localization procedure, the ACC target regions could be functionally defined successfully in all participants and sessions, except for the last session of one control subject (table 2). Overall, localization of ACC target regions was similar across groups, and sessions, all target regions were located within the boundaries of the anterior cingulate cortex (table 2). The coordinates of the individual target regions were as expected based on previous studies with ADHD patients (Bush et al., 2008) (table 2, figure 4a). The post-hoc whole-brain random-effects GLM analysis confirmed that

A

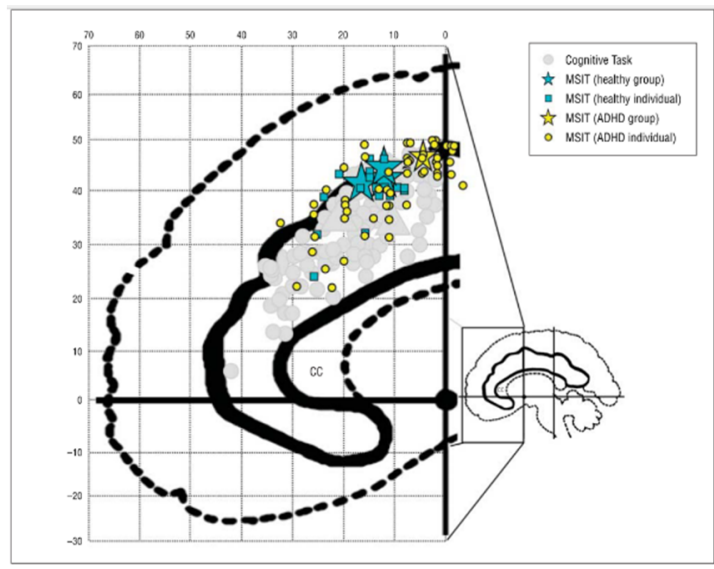

B

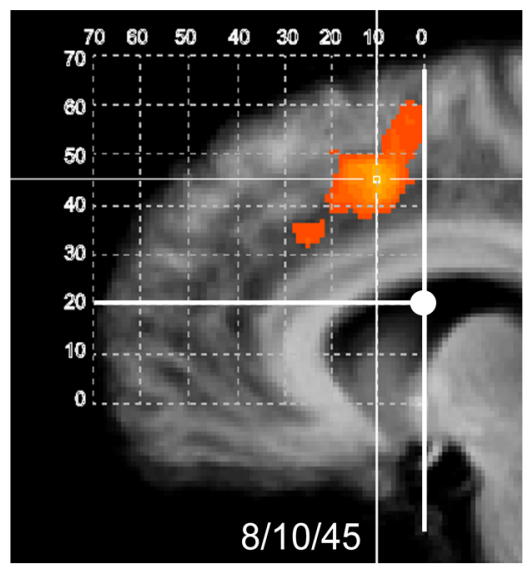

C

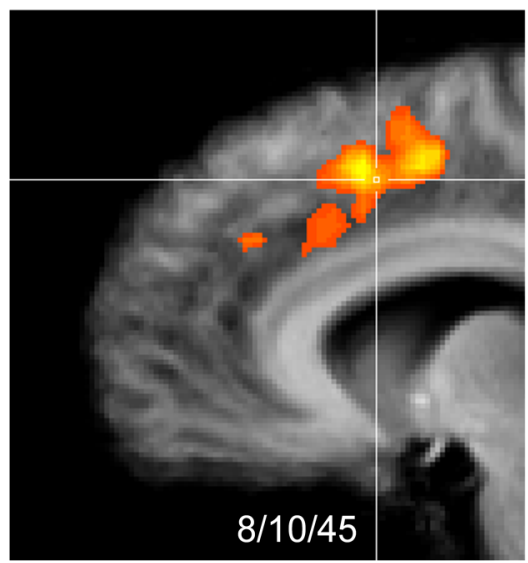

Figure 4. Post-hoc analysis of localization runs.

Part $\mathbf{A}$ of the figure depicts the group and individual activation foci in the dorsal anterior cingulate cortex during performance of the MSIT in previous studies with ADHD patients and healthy controls (printed with permission from Bush et al., 2008). In part B the activation focus $(x=8, y=10, z=45)$, when contrasting the interference and control conditions is depicted (random effects, $p<0.05$, corrected at cluster level). The grid from part $(\mathbf{A})$ is overlaid to show the spatial overlap in effects. Part $\mathbf{C}$ shows that during the second localization task, when participants were performing the mental calculation task, the same region was activated (random effects, $p<0.05$, corrected at cluster level). 
A

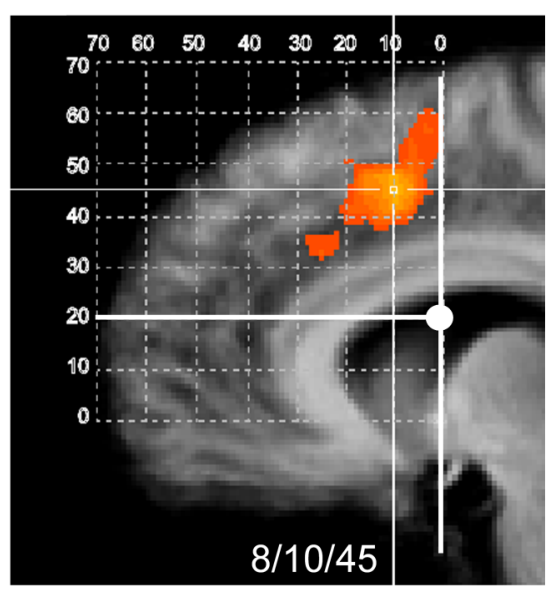

B

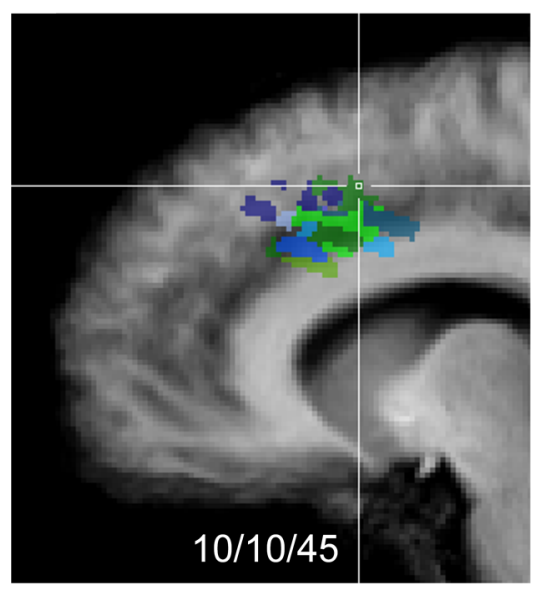

C

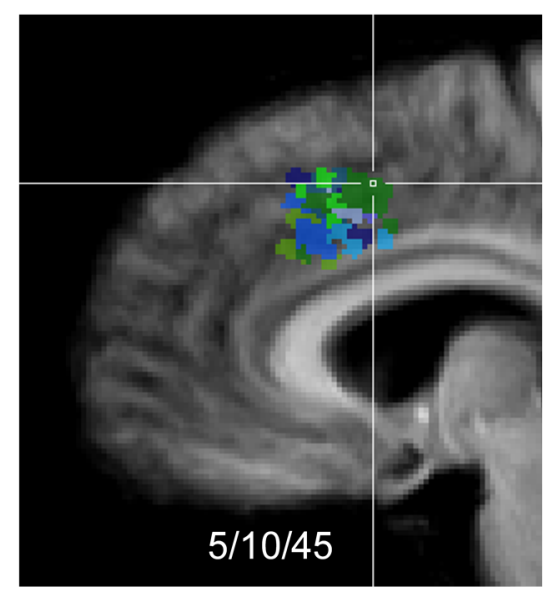

Figure 5. Localization of target regions.

In part $\mathbf{A}$ the region activated when contrasting the interference with the control condition of the MSIT localization task is depicted (random effects, $p<0.05$, corrected at cluster level). In part $\mathbf{B}$ and $\mathbf{C}$ representative individual ACC target regions are shown for neurofeedback participants (blue tints), and control participants (green tints). While the target regions were defined based on the more robust interference versus rest contrast, they showed reasonable overlap with the effect in the post-hoc whole-brain analysis. The slightly more anterior location of the target regions may have resulted from a preference for anterior clusters during the localization procedure, which is the part shown to be under-activated in ADHD patients (Bush et al., 2008).

dorsal anterior cingulate cortex was activated during the MSIT localization task (figure 4b), with an activation peak as described in previous literature $(x=8, y=10, z=45)$ (Bush et al., 2008). It furthermore confirmed that the second localization task, during which the participant did a medium-difficulty mental calculation task, activated the same region (figure $\mathbf{4 b}, \mathbf{c}$ ). The individual $A C C$ target regions showed reasonable overlap with the effect found during the post-hoc GLM whole-brain analysis (figure 5). The post-hoc region-of-interest analysis of activation levels in the target regions showed that for both groups, and both localization tasks there were no significant changes in activation level over sessions.

\section{MRI training runs}

The results from the region-of-interest analysis indicate that participants were able to activate anterior cingulate cortex during the mental calculation task, showing increased activation levels during the task periods (figure 6). For neurofeedback participants 
Table 2.

Talairach coordinates of target regions in Anterior Cingulate Cortex

\begin{tabular}{|c|c|c|c|c|c|c|c|c|c|c|c|c|c|c|}
\hline \multicolumn{15}{|c|}{ Neurofeedback group } \\
\hline & \multicolumn{3}{|c|}{ session 1} & \multicolumn{3}{|c|}{ session 2} & \multicolumn{3}{|c|}{ session 3} & \multicolumn{3}{|c|}{ session 4} & \multicolumn{2}{|c|}{ mean } \\
\hline & $x$ & $y$ & z & $x$ & $y$ & z & $x$ & $y$ & z & $x$ & $y$ & $z$ & $x$ & $y$ \\
\hline S11 & 6 & 21 & 41 & -1 & 16 & 41 & -7 & 15 & 38 & -2 & 17 & 41 & -1 & 17 \\
\hline S12 & 9 & 12 & 42 & 8 & 17 & 39 & 8 & 10 & 41 & 4 & 9 & 41 & 7 & 12 \\
\hline S13 & 3 & 14 & 40 & -2 & 12 & 41 & -3 & 12 & 44 & $x$ & $x$ & $x$ & -1 & 13 \\
\hline S14 & -3 & 24 & 43 & -3 & 23 & 44 & -4 & 1 & 49 & -4 & 2 & 46 & -4 & 13 \\
\hline S15 & 1 & 6 & 31 & 10 & 6 & 34 & 3 & 19 & 34 & 2 & 12 & 34 & 0 & 18 \\
\hline S16 & 4 & 15 & 41 & -3 & 23 & 38 & 1 & 16 & 41 & -1 & 18 & 39 & 5 & 22 \\
\hline S17 & 3 & 21 & 38 & 3 & 20 & 35 & 6 & 23 & 34 & 8 & 22 & 34 & 4 & 11 \\
\hline mean & 3 & 16 & 39 & 2 & 17 & 39 & 1 & 14 & 40 & 1 & 13 & 39 & 2 & 15 \\
\hline SD & 4 & 6 & 4 & 5 & 6 & 4 & 6 & 7 & 5 & 4 & 7 & 5 & 4 & 4 \\
\hline
\end{tabular}

\section{Control group}

\begin{tabular}{llllllllllllllll}
\hline & \multicolumn{1}{c}{ session 1 } & \multicolumn{1}{c}{ session 2 } & \multicolumn{4}{c}{ session 3 } & \multicolumn{3}{c}{ session 4 } & \multicolumn{3}{c}{ mean } \\
\hline & $\mathrm{x}$ & $\mathrm{y}$ & $\mathrm{z}$ & $\mathrm{x}$ & $\mathrm{y}$ & $\mathrm{z}$ & $\mathrm{x}$ & $\mathrm{y}$ & $\mathrm{z}$ & $\mathrm{x}$ & $\mathrm{y}$ & $\mathrm{z}$ & $\mathrm{x}$ & $\mathrm{y}$ \\
\hline S21 & 1 & 18 & 41 & -1 & 13 & 35 & 4 & 14 & 39 & 3 & 11 & 44 & 2 & 14 \\
S22 & 8 & 22 & 31 & -2 & -1 & 34 & 5 & 27 & 39 & 5 & 27 & 39 & 4 & 16 \\
S23 & 5 & 14 & 40 & 5 & 10 & 42 & 4 & 21 & 37 & 7 & 13 & 41 & 5 & 15 \\
\hline mean & $\mathbf{5}$ & $\mathbf{1 8}$ & $\mathbf{3 7}$ & $\mathbf{1}$ & $\mathbf{7}$ & $\mathbf{3 7}$ & $\mathbf{4}$ & $\mathbf{2 1}$ & $\mathbf{3 8}$ & $\mathbf{5}$ & $\mathbf{1 2}$ & $\mathbf{4 3}$ & $\mathbf{4}$ & $\mathbf{1 5}$ \\
\hline SD & 4 & 4 & 6 & 4 & 7 & 4 & 1 & 7 & 1 & 3 & 1 & 2 & 2 & 1 \\
\hline
\end{tabular}

Based on the localization procedure, the ACC target regions could be successfully defined in all participants and sessions, except for the last session of one control subject (S16). In this case, the target region from the previous session was used. One participant from the neurofeedback group did not participate in the last session (S13). Overall, the location of the ACC target regions was similar across groups, and sessions, and as expected based on previous studies (Bush et al., 2008).

activation levels during cognitive training runs increased over sessions, being significantly higher during the third session in comparison to the second session $(F(1,6)$ $=6.6, p<0.05, \eta_{p}^{2}=0.52$, figure 6). The same effect was apparent for the transfer run, during which they did not receive feedback $\left(F(1,6)=8.0, p<0.05, \eta_{p}^{2}=0.57\right.$, figure 6). Control participants showed the opposite development of activation levels over time, with decreasing activation levels in the last two sessions (figure 6). Both 
A

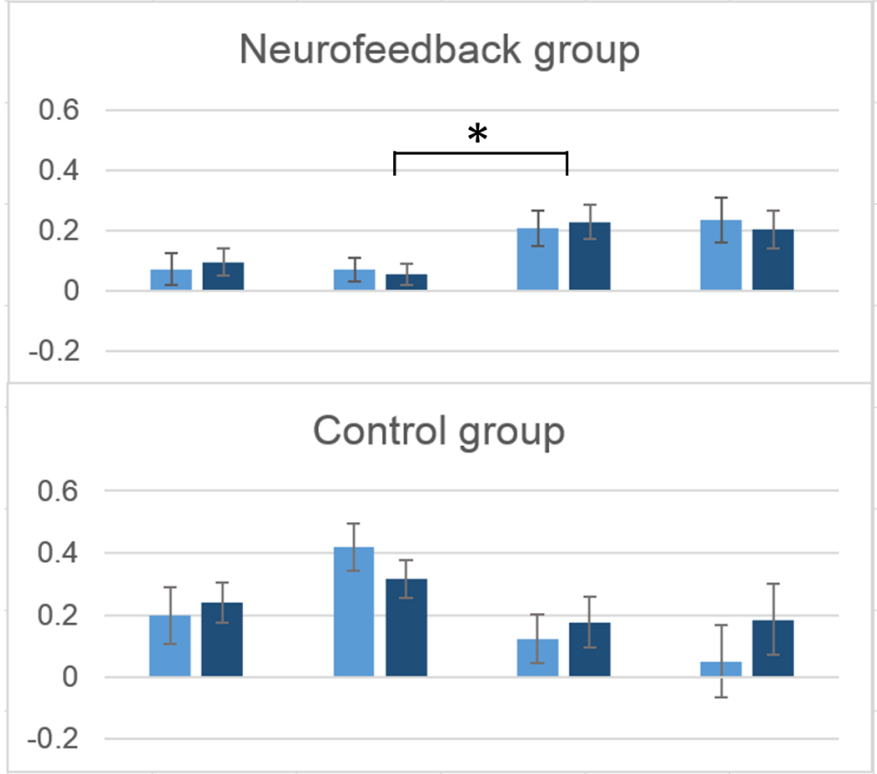

Session 1 Session 2 Session $3 \quad$ Session 4

$50 \%$ difficulty $\square 100 \%$ difficulty
C

Neurofeedback group

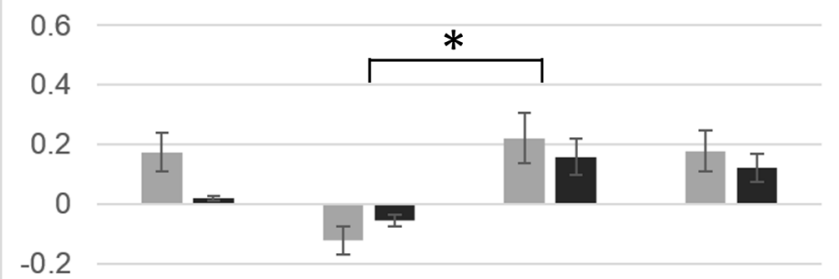

D

Control group

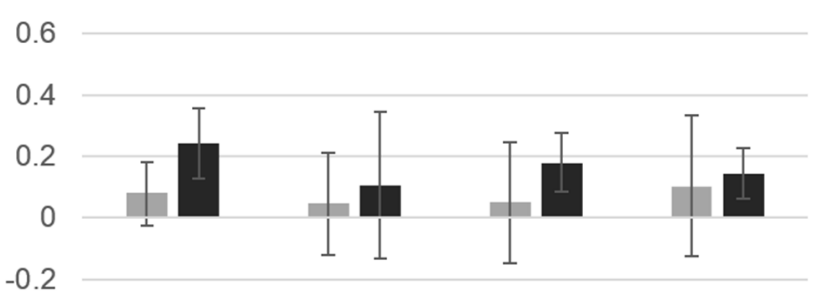

Session 1 Session 2 Session 3 Session 4

$50 \%$ difficulty
$100 \%$ difficulty

\section{Figure 6. BOLD response in target regions during training.}

The results from the region-of-interest analysis for the defined target regions indicated that participants were able to activate anterior cingulate cortex during the mental calculation task $(\mathbf{A}, \mathbf{B})$. In the neurofeedback group, activation levels during the first two sessions were low, but increased significantly after the second session (A). For control participants, the development over time was the opposite, starting with high activation levels during the first two sessions, and showing a drop in response during later sessions (B). Both groups did not achieve differential modulation of the two different task conditions (50\% difficulty, $100 \%$ difficulty), and did not show improvement over sessions (A, B). A similar picture emerged from the analysis of the transfer runs of each session, during which both groups did not received feedback. While neurofeedback participants showed increased activation levels after the second session (C), there was no such increase over time for control participants (D).

groups did not achieve differential modulation for the two task conditions (50\% difficulty, 100\% difficulty), and did not show improvement over sessions (figure 6).

The analysis of the data from the Questionnaire of Current Motivation (QCM) showed that perceived challenge decreased linearly within control participants over sessions $\left(F(1,2)=64.0 p<0.05, \eta_{p}^{2}=0.97\right.$, figure 7$)$. There were no within group changes over time for any other motivational factor. The analysis of predictors of 

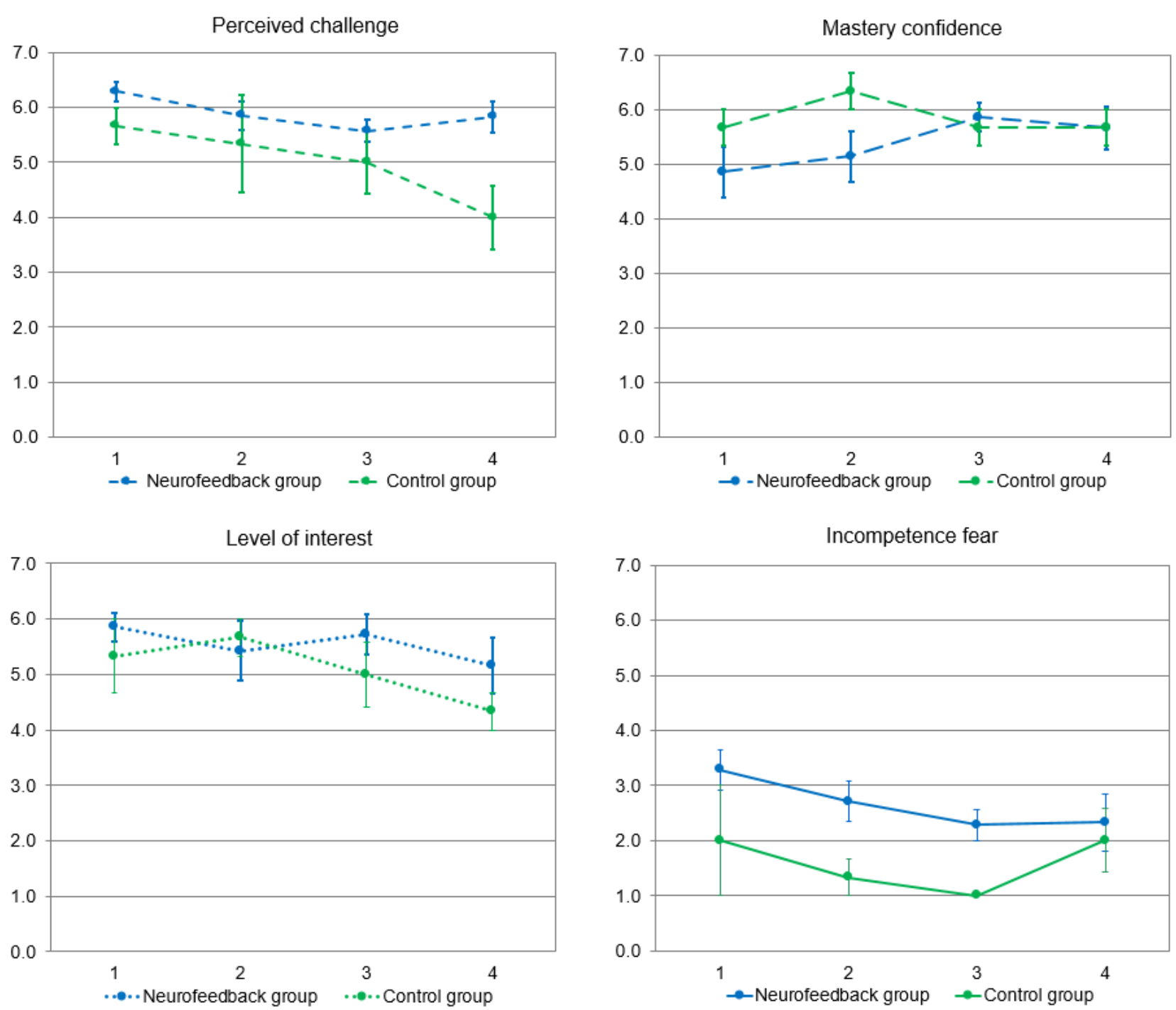

Figure 7. Results regarding participants' motivation.

The scores from the Questionnaire of Current Motivation (QCM) are depicted for each session (1-4) for the neurofeedback (blue), and control group (green). The only significant change over time regarding any of the four different motivational factors (incompetence fear, perceived challenge, level of interest, mastery confidence), was a linear decrease of perceived challenge over session for the control participants. All motivational factors were measured on a Likert Scale (1-7).

individual performance during cognitive training runs showed that ability to employ attention for controlling response inhibition was highly correlated with general performance in both groups (table 3, figure 8). For neurofeedback participants a significant correlation with improvement across sessions was found for ability to employ attention for controlling response inhibition, and visual working memory capacity (table 3, figure 8). 
A

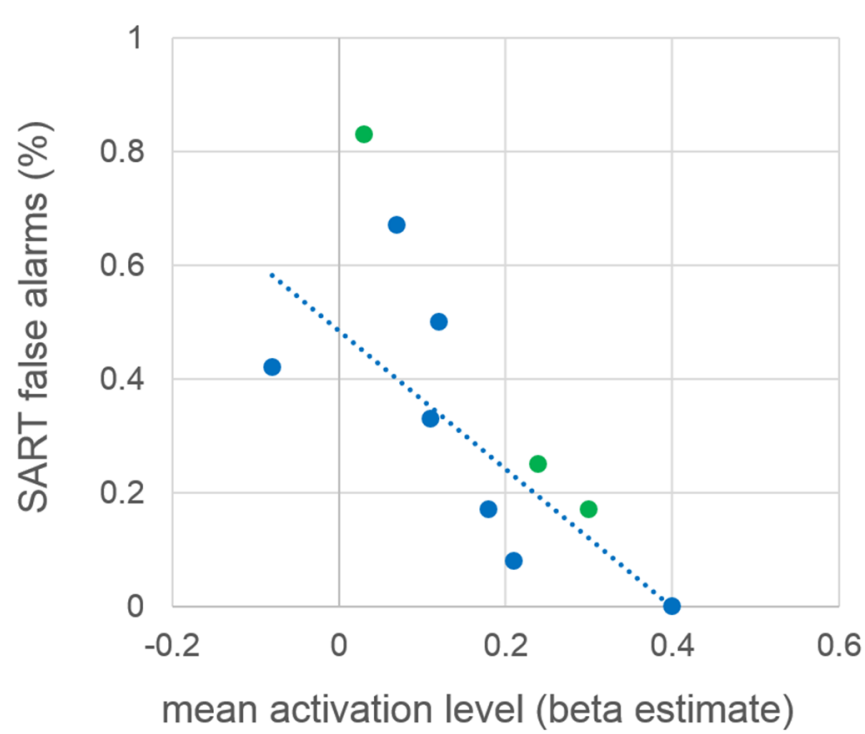

B

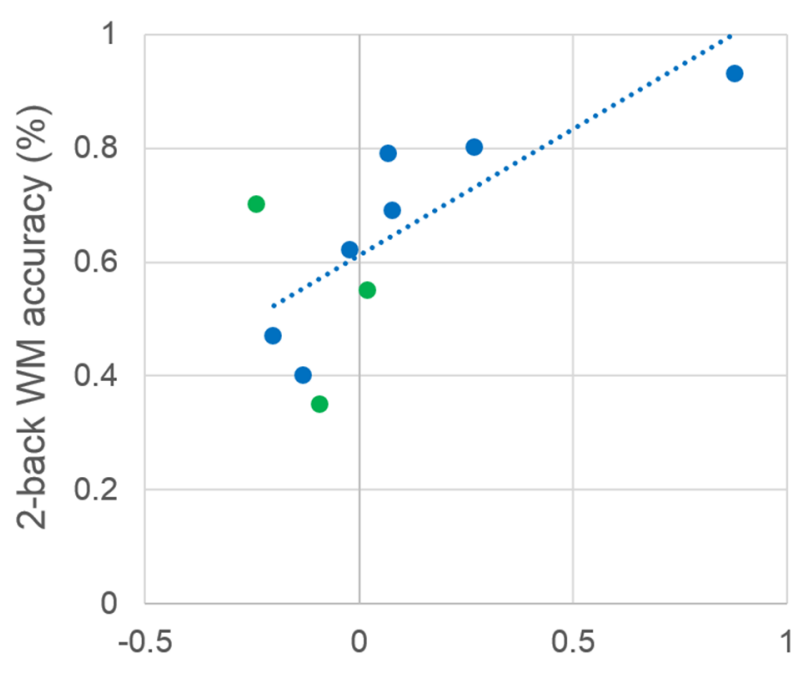

increase activation level (beta estimate)

Figure 8. Predictors of individual modulation performance.

General task performance during the cognitive training runs, as measured by mean activation level, showed a high correlation (neurofeedback group: 0.74 ) with ability to sustained attention during pre-testing (SART false alarms, A). Participants with a lower percentage of false alarms during pre-testing, achieved higher mean activation levels during the later training (neurofeedback participants $=$ blue dots/regression line, control participants $=$ green dots) . Second, a significant correlation (neurofeedback group: 0.85 ) between visual working memory capacity (2-back WM accuracy), and improvement across sessions was found for neurofeedback participants (B). Better visual working memory during pre-testing was correlated with stronger improvement over sessions. This correlation remained significant when the extreme value was removed (neurofeedback participants = blue dots/regression line, control participants $=$ green dots). 
Table 3. Predictors of individual modulation performance

\begin{tabular}{l|l|l|}
\hline $\begin{array}{l}\text { Predictors } \\
\text { (score pre-testing) }\end{array}$ & $\begin{array}{l}\text { Control group }(n=3) \\
\text { correlation }(p \text {-value })\end{array}$ & $\begin{array}{l}\text { Neurofeedback group }(n=7) \\
\text { correlation ( } p \text {-value) }\end{array}$ \\
\hline
\end{tabular}

Correlation with general task performance (mean activation level)

\begin{tabular}{|l|l|l|}
\hline SA-DOTS false alarms & $\mathbf{1 . 0 0}(p=0.02) *$ & $0.63(p=0.13)$ \\
\hline SA-DOTS missed targets & $0.98(p=0.12)$ & $0.27(p=0.56)$ \\
\hline SART false alarms & $0.99(p=0.07)$ & $0.74(p=0.06)$ \\
\hline 2-back WM accuracy & $0.92(p=0.25)$ & $0.41(p=0.36)$ \\
\hline WAIS WM & $0.13(p=0.92)$ & $0.05(p=0.92)$ \\
\hline
\end{tabular}

Correlation with improvement over sessions (activation increase)

\begin{tabular}{l|l|l}
\hline SA-DOTS false alarms & $0.78(p=0.43)$ & $0.53(p=0.22)$ \\
\hline SA-DOTS missed targets & $0.89(p=0.29)$ & $0.33(p=0.47)$ \\
\hline SART false alarms & $0.85(p=0.35)$ & $\mathbf{0 . 8 0}(p=0.03) *$ \\
\hline 2-back WM accuracy & $0.51(p=0.66)$ & $\mathbf{0 . 8 5}(p=0.02)$ * \\
\hline WAIS WM & $0.50(p=0.67)$ & $0.25(p=0.59)$ \\
\hline
\end{tabular}

Correlation with improvement in modulation (increase differential modulation)

\begin{tabular}{l|l|l|}
\hline SA-DOTS false alarms & $0.97(p=0.11)$ & $0.07(p=0.88)$ \\
\hline SA-DOTS missed targets & $0.93(p=0.24)$ & $\mathbf{0 . 7 4}(\mathbf{p}=\mathbf{0 . 0 6})$ \\
\hline SART false alarms & $0.96(p=0.19)$ & $0.13(p=0.77)$ \\
\hline 2-back WM accuracy & $0.98(p=0.13)$ & $0.35(p=0.45)$ \\
\hline WAIS WM & $0.32(p=0.79)$ & $\mathbf{0 . 6 7}(\mathbf{p}=\mathbf{0 . 0 9})$ \\
\hline
\end{tabular}

Significant effects $(p \leq 0.05)$ are printed bold and marked with an asterisk.

Trends towards significance $(p \leq 0.10)$ are printed bold.

The analysis of the predictors of individual modulation performance during cognitive training runs showed that working memory capacity, and ability to sustain attention during pre-testing were highly correlated with later modulation performance. General task performance, as measured by mean activation level, was highly correlated with measures of sustained attention indexing vigilance and response inhibition (SA-DOTS false alarm, SART false alarms) in both groups. In neurofeedback participants, improvement over sessions, was significantly correlated with their ability to employ attentional control for response inhibition (SART false alarms), and visual working memory capacity (2-back WM accuracy). There was also a high correlation of response inhibition (SA-DOTS missed targets), and verbal working memory (WAIS WM) scores with improvement of differential modulation over sessions. 


\section{Pre-post behavioral assessment}

During pre-testing participants reported on average $7.2(S D=1.1)$ out of 9 possible ADHD symptoms of attention, and 6.6 (SD = 2.5) out of 9 possible ADHD symptoms of impulsivity (table 4). On the age-normed test of sustained attention (SA-DOTS) participants scored 1.2 standard scores $(S D=1.7)$ below their age group on vigilance, and 2.4 standard scores $(S D=3.0)$ below age-normed performance on response inhibition (table 4). On the sustained attention response test (SART), they had a false alarm rate of $34 \%$ of the trials (SD $=26 \%$ ), in comparison to $12 \%$ in a healthy control group performing the same task (O'Connell et al., 2008) (table 4). Participants scored

\section{Table 4. Pre-post behavioral assessment}

\begin{tabular}{|c|c|c|}
\hline $\begin{array}{l}\text { Variables assessed during } \\
\text { pre-l post-testing }\end{array}$ & $\begin{array}{l}\text { Control group }(n=3) \\
\text { Pre-testing/post-testing } \\
\left.\text { p-value (partial eta }{ }^{2}\right)\end{array}$ & $\begin{array}{l}\text { Neurofeedback group }(n=7) \\
\text { Pre-testing/post-testing } \\
\left.\text { p-value (partial eta }{ }^{2}\right)\end{array}$ \\
\hline $\begin{array}{l}\text { ADHD attention score } \\
\text { (DSM-IV rating scale) }\end{array}$ & $\begin{array}{ll}\text { pre: } 7.7 & \text { post: } 7.0 \\
p=0.18 & (0.67)\end{array}$ & $\begin{array}{ll}\text { pre: } 7.0 & \text { post: } 6.0 \\
p=0.23 & (0.23)\end{array}$ \\
\hline $\begin{array}{l}\text { ADHD impulsivity score } \\
\text { (DSM-IV rating scale) }\end{array}$ & $\begin{array}{ll}\text { pre: } 7.0 & \text { post: } 5.3 \\
p=0.30 & (0.49)\end{array}$ & $\begin{array}{ll}\text { pre: } 6.4 & \text { post: } 5.7 \\
p=0.31 & (0.17)\end{array}$ \\
\hline $\begin{array}{l}\text { SA-DOTS false alarms } \\
\text { (age-referenced z-score) }\end{array}$ & $\begin{array}{ll}\text { pre: } 2.2 & \text { post: } 1.1 \\
p=0.01 & (0.97)^{*}\end{array}$ & $\begin{array}{ll}\text { pre: } 0.8 & 0.1 \\
p=0.10 & (0.39)\end{array}$ \\
\hline $\begin{array}{l}\text { SA-DOTS missed targets } \\
\text { (age-referenced z-score) }\end{array}$ & $\begin{array}{ll}\text { pre: } 1.5 & \text { post } 0.7 \\
p=0.27 & (0.53)\end{array}$ & $\begin{array}{ll}\text { pre: } 2.8 & \text { post: } 1.1 \\
p=0.05 & (0.49) *\end{array}$ \\
\hline $\begin{array}{l}\text { SART false alarms } \\
(\%)\end{array}$ & $\begin{array}{ll}\text { pre: } 42 \% & \text { post: } 31 \% \\
p=0.38 & (0.38)\end{array}$ & $\begin{array}{l}\text { pre: } 31 \% \text { post: } 31 \% \\
p=1.00 \quad(0.00)\end{array}$ \\
\hline $\begin{array}{l}\text { 2-back WM accuracy } \\
(\%)\end{array}$ & $\begin{array}{l}\text { pre: } 53 \% \text { post: } 59 \% \\
p=0.67 \quad(0.11)\end{array}$ & $\begin{array}{l}\text { pre: } 67 \% \text { post: } 76 \% \\
p=0.03 \quad(0.56) *\end{array}$ \\
\hline $\begin{array}{l}\text { WAIS WM } \\
\text { (age-referenced IQ-score) }\end{array}$ & $\begin{array}{ll}\text { pre: } 98 & \text { post: } 112 \\
p=0.21 & (0.63)\end{array}$ & $\begin{array}{ll}\text { pre: } 96 & \text { post: } 101 \\
p=0.41 & (0.12)\end{array}$ \\
\hline
\end{tabular}

Significant effects $(p \leq 0.05)$ are printed bold and marked with an asterisk.

Trends towards significance $(p \leq 0.10)$ are printed bold.

While ADHD attention and impulsivity symptoms were reduced during post-testing, these changes did not reach significance. Neuropsychological testing of cognitive functioning showed that after the neurofeedback training working memory capacity (2-back WM accuracy), and ability to employ attention to control response inhibition (SA-DOTS missed targets) was significantly improved in neurofeedback participants. There was also a trend for improved vigilance (SA-DOTS false alarms) in the neurofeedback group, which reached significance in control participants. 
A

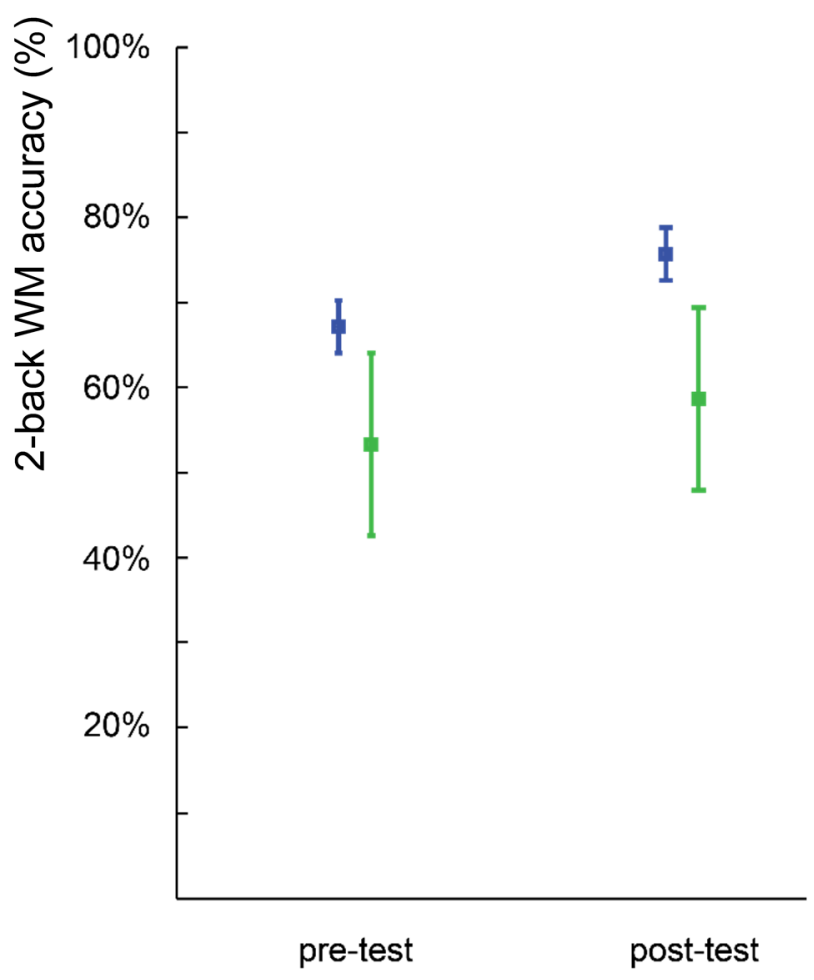

B

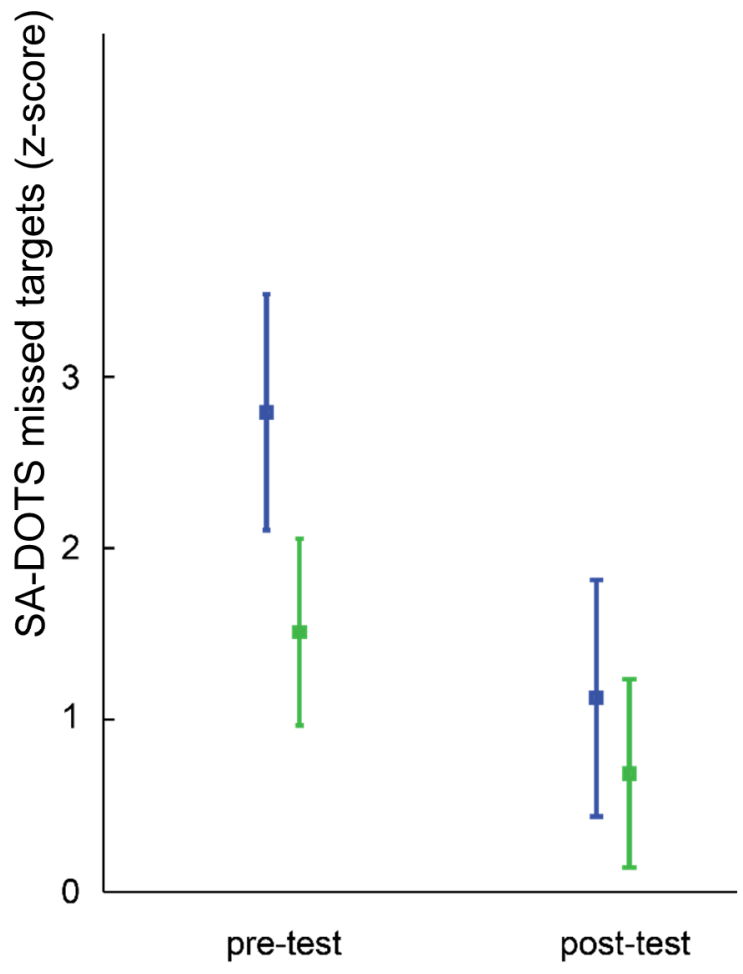

Figure 9. Pre-post assessment

Analysis of behavioral pre-post assessment revealed significant changes in the neurofeedback group (neurofeedback group $=$ blue, control group $=$ green $)$. Visual working memory capacity (2-back WM accuracy) was significantly improved in neurofeedback participants, who showed higher overall accuracy during post-testing (A). Second, there was a significant effect for improved ability to employ attention during response inhibition (SADOTS missed targets), as indexed by a reduction in the number of missed targets (B).

an average of $97 \mathrm{IQ}$ points $(S D=11.1)$ on the standardized WAIS verbal working memory test, and achieved $63 \%$ accuracy $(S D=19 \%)$ in the visual working memory task (2-back WM), compared to $78 \%$ accuracy reported for healthy controls for the same task (Hoogman, 2012) (table 4).

During post-testing ADHD inattention and hyperactivity/impulsivity symptoms were reduced respective to pre-testing, however, this treatment change did not reach significance (table 4). During neuropsychological testing, participants showed significant advancement on several measures of cognitive functioning. There was a trend towards significance for improved vigilance in the neurofeedback group (SADOTS false alarms: -0.7 standard scores), which reached significance for control 
participants (SA-DOTS false alarms: -1.1 standard scores, table 4). Also, in neurofeedback participants there was a significant, and large improvement in the ability to employ attention control during response inhibition (SA-DOTS missed targets: -1.7 standard scores), and consistent improvement of visual working memory capacity (2back WM accuracy: $+9 \%$, table 4, figure 9). Overall, both groups improved on all measures of ADHD symptoms and cognitive functioning (table 4). This positive treatment change was only moderately correlated with individual performance during cognitive training runs (table 5), and significantly correlated with baseline measures of sustained attention. Participants who had the strongest impairments during pre-testing also showed the greatest improvements (table 5). Overall, participants improved both regarding ADHD symptoms and cognitive functioning, even when showing strong impairment during pre-testing.

\section{Table 5. Predictors of treatment change}

\begin{tabular}{l|l|l}
\hline $\begin{array}{l}\text { Change as assessed by } \\
\text { pre-l post-testing }\end{array}$ & $\begin{array}{l}\text { Control group }(n=3) \\
\text { correlation }(p-v a l u e)\end{array}$ & $\begin{array}{l}\text { Neurofeedback group }(n=7) \\
\text { correlation ( } p \text {-value) }\end{array}$ \\
\hline
\end{tabular}

Correlation with individual modulation performance (improvement over sessions)

\begin{tabular}{|l|l|l|l|}
\hline SA-DOTS false alarms & $1.00 \quad(p=0.003) *$ & $0.42 \quad(p=0.36)$ \\
\hline SA-DOTS missed targets & $0.66 \quad(p=0.54)$ & $0.42 \quad(p=0.35)$ \\
\hline 2-back WM accuracy & $0.94 \quad(p=0.22)$ & $0.58 \quad(p=0.17)$
\end{tabular}

Correlation with pre-assessment

\begin{tabular}{|l|l|l|l|}
\hline SA-DOTS false alarms & $0.78 \quad(p=0.43)$ & $\mathbf{0 . 8 0} \quad(p=0.03) *$ \\
\hline SA-DOTS missed targets & $0.94 \quad(p=0.20)$ & $\mathbf{0 . 8 6} \quad(p=0.01)$ * \\
\hline 2-back WM accuracy & $0.19 \quad(p=0.88)$ & $0.54 \quad(p=0.22)$ \\
\hline
\end{tabular}

Significant effects $(p \leq 0.05)$ are printed bold and marked with an asterisk.

Trends towards significance $(p \leq 0.10)$ are printed bold.

Evaluation of predictors of treatment change showed that in neurofeedback participants, treatment change was only moderately correlated with individual modulation performance during cognitive training runs. Second, there was a significant, positive correlation between response inhibition and vigilance at baseline and treatment change on these variables. Participants showing the strong impairments during pre-testing also showed the greatest improvement. 


\section{Discussion}

The primary objective was to evaluate if the fMRI-neurofeedback training was successful in reducing ADHD symptoms and improving cognitive functioning. Thus, the first goal was to show if ADHD patients were able to voluntarily modulate their individual anterior cingulate cortex activation level. The second goal was to assess whether this modulation had a specific influence on ADHD symptoms and the performance during cognitive tasks. Finally, it was evaluated which were the moderating factors for treatment success.

Included participants showed high levels of impairment, both regarding ADHD symptoms and during neuropsychological testing (de Sonneville, 2005; Hoogman, 2012; Kooij et al., 2005; O'Connell et al., 2008; Spencer, Biederman, \& Mick, 2007). Analysis of activation levels in dorsal ACC target regions during cognitive training showed that participants were generally able to activate the target region in anterior cingulate cortex, but did not achieve differential modulation between conditions, and did not improve regarding differential modulation. Neurofeedback participants, however, did show up-regulation of activation levels over sessions. While cingulate cortex activation was low during the first two sessions, when participants possibly had to adapt to the dual task of monitoring feedback and performing the training task, it was significantly increased during the later sessions. This finding closely resembles previous results showing that patients needed several training sessions before they adapt to the task, and show increased activation levels (Linden et al., 2012; Scheinost et al., 2013). Importantly, control participants did not show an up-regulation effect over time. While this result is preliminary, due to the low number of control participants having completed the experiment, it is a first indication that control participants did not learn to up-regulate equally well. A possible explanation for this may be that a decrease of perceived challenge during the later sessions, however, there were no strong differences in motivation between the groups until the last session, therefore this aspect would not explain the differential development over time entirely. Other factors that may explain modulation success on an individual level were ability to exert attentional control, and visual working memory capacity, which were highly correlated with modulation success. These results confirm previous research on individual predictors of control during EEG-neurofeedback, also showing that working memory capacity and attentional control predicted regulation success (Daum et al., 1993; 
Holzapfel, 1998). As participants with high initial levels of impairments were less successful during modulation, neurofeedback trainings may be more suited for this patient group if target levels would be individualized depending on the initial level of impairment. Also, step-wise procedures that are more directly targeted at facilitating up-regulation over sessions, for example by increasing target levels gradually, may be simpler, and therefore more suited for this patient group. Finally, a more tailored approach could include a pre-training phase, during which participants learn how to regulate the BOLD signal of a brain region that is easily controllable by simple cognitive strategies, as for example regions in the motor network, in which activation levels can be modulated by motor imagery.

Assessment of treatment change showed that ADHD symptoms were reduced after the training, without reaching a significant treatment effect. Cognitive functioning did improved significantly in neurofeedback participants, with ability to control attention improving considerably (de Sonneville, 2005), and visual working memory capacity reaching levels that have been measured in healthy controls (Hoogman, 2012). Treatment changes were only moderately correlated with modulation success, suggesting that either cognitive treatment per se may have had a beneficial effect or that indices of modulation success did not adequately capture individual learning. The contribution of the provided neurofeedback information to treatment change cannot be finally evaluated based on the presented data, between-group comparisons would be necessary for a final evaluation of the efficacy of neurofeedback training.

In general, it can be concluded that the implemented training had positive effects on ADHD symptoms and cognitive functioning, even in participants with initial high impairments. It needs to be further investigated if the provided neurofeedback information was a causal factor. Another future goal will be investigating if the efficacy of the training can be further increased by implementing procedures tailored to the target group. 


\section{Acknowledgements}

We want to thank Anita Kaemingk, Leonie Hennissen, and Tabea Kamp for their dedicated support with organizational issues, Bjoern Zierul for his work during the pilot measurements, and Silja Timm for programming behavioral data analysis tools. The authors gratefully acknowledge the support of the BrainGain Smart Mix Program of The Netherlands Ministry of Economic Affairs and The Netherlands Ministry of Education, Culture and Science (grant number: SSM06011). 


\section{References}

American Psychiatric Association. (2000). Diagnostic and Statistical Manual of Mental Disorders, Fourth Edition, Text Revision (DSM-IV-TR). Text (Vol. 1). Washington, DC: American Psychiatric Publishing. doi:10.1176/appi.books.9780890423349

American Psychiatric Association. (2013). Diagnostic and Statistical Manual of Mental Disorders, Fifth Edition. Arlington. Washington, DC: American Psychiatric Publishing. doi:10.1176/appi.books.9780890425596.744053

Borm, G. F., Hoogendoorn, E. H., den Heijer, M., \& Zielhuis, G. a. (2005). Sequential balancing: a simple method for treatment allocation in clinical trials. Contemporary Clinical Trials, 26(6), 637-45. doi:10.1016/j.cct.2005.09.002

Bron, T. I., Bijlenga, D., Marije Boonstra, A., Breuk, M., Pardoen, W. F. H., Beekman, A. T. F., \& Sandra Kooij, J. J. (2014). OROS-methylphenidate efficacy on specific executive functioning deficits in adults with ADHD: A randomized, placebocontrolled cross-over study. European Neuropsychopharmacology, 24(4), 519528. doi:10.1016/j.euroneuro.2014.01.007

Bush, G. (2011). Cingulate, frontal, and parietal cortical dysfunction in attentiondeficit/hyperactivity disorder. Biological Psychiatry, 69(12), 1160-7. doi:10.1016/j.biopsych.2011.01.022

Bush, G., Frazier, J. A., Rauch, S. L., Seidman, L. J., Whalen, P. J., Jenike, M. a, ... Biederman, J. (1999). Anterior cingulate cortex dysfunction in attentiondeficit/hyperactivity disorder revealed by $\mathrm{fMRI}$ and the Counting Stroop. Biological Psychiatry, 45(12), 1542-52. Retrieved from http://www.ncbi.nlm.nih.gov/pubmed/10376114

Bush, G., \& Shin, L. M. (2006). The Multi-Source Interference Task: an fMRI task that reliably activates the cingulo-frontal-parietal cognitive/attention network. Nature Protocols, 1(1), 308-313.

Bush, G., Shin, L. M., Holmes, J., Rosen, B. R., \& Vogt, B. a. (2003). The Multi-Source Interference Task: validation study with $\mathrm{fMRI}$ in individual subjects. Molecular Psychiatry, 8(1), 60-70. doi:10.1038/sj.mp.4001217

Bush, G., Spencer, T. J., Holmes, J., Shin, L. M., Valera, E. M., Seidman, L. J., ... Biederman, J. (2008). Functional magnetic resonance imaging of methylphenidate and placebo in attention-deficit/hyperactivity disorder during the multi-source interference task. Archives of General Psychiatry, 65(1), 102-14. doi:10.1001/archgenpsychiatry.2007.16

Coghill, D. R., Rhodes, S. M., \& Matthews, K. (2007). The neuropsychological effects of chronic methylphenidate on drug-naive boys with attention-deficit/hyperactivity disorder. Biological Psychiatry, 62(9), 954-62. doi:10.1016/j.biopsych.2006.12.030 
Cohen, J. (1973). Eta-Squared and Partial Eta-Squared in Fixed Factor Anova Designs. Educational and Psychological Measurement, 33(1), 107-112. doi:10.1177/001316447303300111

Cotan. (2012). Wechsler Adult Intelligence Scale, Fourth Edition, Nederlandstalige Bewerking (WAIS-IV-NL) (4th ed.). Amsterdam, The Netherlands: Pearson.

Cubillo, A., Halari, R., Ecker, C., Giampietro, V., Taylor, E., \& Rubia, K. (2010). Reduced activation and inter-regional functional connectivity of fronto-striatal networks in adults with childhood Attention-Deficit Hyperactivity Disorder (ADHD) and persisting symptoms during tasks of motor inhibition and cognitive switching. Journal of Psychiatric Research, 44(10), 629-39. doi:10.1016/j.jpsychires.2009.11.016

Daum, I., Rockstroh, B., Birbaumer, N., Elbert, T., Canavan, A., \& Lutzenberger, W. (1993). Behavioural treatment of slow cortical potentials in intractable epilepsy: neuropsychological predictors of outcome. Journal of Neurology, Neurosurgery, and Psychiatry, 56(1), 94-7.

De Sonneville, L. M. J. (1999). Amsterdam Neuropsychological Tasks: a computeraided assessment program. In B. P. L. M. Den Brinker, P. J. Beek, A. N. Brand, S. J. Maarse, \& L. J. M. Mulder (Eds.), Computers in Psychology, Vol. 6: Cognitive Ergonomics, Clinical Assessment and Computer-Assisted Learning (pp. 187203). Lisse, The Netherlands: Swets \& Zeitlinger.

De Sonneville, L. M. J. (2005). (Amsterdam Neuropsychological Tasks: Scientific and Clinical Applications, in Dutch). Amsterdamse Neuropsychologische Taken: Wetenschappelijke en klinische toepassingen. Tijdschrift Voor Neuropsychologie, $0,27-41$.

DeCharms, R. C., Maeda, F., Glover, G. H., Ludlow, D., Pauly, J. M., Soneji, D., ... Mackey, S. C. (2005). Control over brain activation and pain learned by using realtime functional MRI. Proceedings of the National Academy of Sciences of the United States of America, 102(51), 18626-31. doi:10.1073/pnas.0505210102

Delazer, M., Domahs, F., Bartha, L., Brenneis, C., Lochy, a, Trieb, T., \& Benke, T. (2003). Learning complex arithmetic-an fMRI study. Cognitive Brain Research, 18(1), 76-88. doi:10.1016/j.cogbrainres.2003.09.005

Dickstein, S. G., Bannon, K., Castellanos, F. X., \& Milham, M. P. (2006). The neural correlates of attention deficit hyperactivity disorder: an ALE meta-analysis. Journal of Child Psychology and Psychiatry, and Allied Disciplines, 47(10), 1051-62. doi:10.1111/j.1469-7610.2006.01671.x

DuPaul, G. J., Power, T. J., Anastopoulus, A. D., \& Reid, R. (1998). ADHD Rating Scale-IV: Checklists, Norms, and Clinical Interpretation. New York: The Guilford Press.

Faraone, S. V, \& Glatt, S. J. (2010). A comparison of the efficacy of medications for adult attention-deficit/hyperactivity disorder using meta-analysis of effect sizes. The Journal of Clinical Psychiatry, 71(6), 754-63. doi:10.4088/JCP.08m04902pur 
Fehr, T., Code, C., \& Herrmann, M. (2007). Common brain regions underlying different arithmetic operations as revealed by conjunct fMRI-BOLD activation. Brain Research, 1172, 93-102. doi:10.1016/j.brainres.2007.07.043

Forman, S. D., Cohen, J. D., Fitzgerald, M., Eddy, W. F., Mintun, M. a, \& Noll, D. C. (1995). Improved assessment of significant activation in functional magnetic resonance imaging (fMRI): use of a cluster-size threshold. Magnetic Resonance in Medicine, 33(5), 636-47.

Goebel, R., Esposito, F., \& Formisano, E. (2006). Analysis of functional image analysis contest (FIAC) data with brainvoyager QX: From single-subject to cortically aligned group general linear model analysis and self-organizing group independent component analysis. Human Brain Mapping, 27(5), 392-401. doi:10.1002/hbm.20249

Goebel, R., Zilverstand, A., \& Sorger, B. (2010). Real-time fMRI-based brain-computer interfacing for neurofeedback therapy and compensation of lost motor functions. Imaging in Medicine, 2(4), 407-415. doi:10.2217/iim.10.35

Graham, J., Banaschewski, T., Buitelaar, J., Coghill, D., Danckaerts, M., Dittmann, R. W., ... Taylor, E. (2011). European guidelines on managing adverse effects of medication for ADHD. European Child \& Adolescent Psychiatry, 20(1), 17-37.

Haller, S., Birbaumer, N., \& Veit, R. (2010). Real-time fMRI feedback training may improve chronic tinnitus. European Radiology, 20(3), 696-703. doi:10.1007/s00330-009-1595-z

Holzapfel, S. (1998). (Predictors of Selfregulation of Slow Cortical Potentials in Epilepsy, in German). Prädiktoren der Selbstregulation der langsamen Hirnpotentiale bei Epilepsie. Tübingen, Germany: Eberhards Karls Universität.

Hoogman, M. (2012). DRD4 and working memory in ADHD patients and healthy subjecs: differential effects on behavior and prefrontal cortex activity. In Imaging the effects of $A D H D$ risk genes (pp. 97-116). Nijmegen, The Netherlands: Donders Series.

Iverson, G. L. (2001). Interpreting change on the WAIS-III/WMS-III in clinical samples. Archives of Clinical Neuropsychology: The Official Journal of the National Academy of Neuropsychologists, 16(2), 183-91. Retrieved from http://www.ncbi.nlm.nih.gov/pubmed/14590186

Kessler, R. C., Adler, L., Barkley, R., Biederman, J., Conners, C. K., Demler, O., ... Zaslavsky, A. M. (2006). The prevalence and correlates of adult ADHD in the United States: results from the National Comorbidity Survey Replication. The American Journal of Psychiatry, 163(4), 716-723.

Kooij, J. J. S. (2003). (ADHD in adults. Introduction to diagnostic assessment and treatment, in Dutch). $A D H D$ bij volwassenen. Inleiding in diagnostiek en behandeling. (2nd ed.). Lisse, The Netherlands: Swets \& Zeitlinger.

Kooij, J. J. S., Buitelaar, J. K., van den Oord, E. J., Furer, J. W., Rijnders, C. a T., \& Hodiamont, P. P. G. (2005). Internal and external validity of attention-deficit 
hyperactivity disorder in a population-based sample of adults. Psychological Medicine, 35(6), 817-27.

Linden, D. E. J., Habes, I., Johnston, S. J., Linden, S., Tatineni, R., Subramanian, L., ... Goebel, R. (2012). Real-time self-regulation of emotion networks in patients with depression. PloS One, 7(6), e38115. doi:10.1371/journal.pone.0038115

Marchetta, N. D. J., Hurks, P. P. M., De Sonneville, L. M. J., Krabbendam, L., \& Jolles, J. (2008). Sustained and focused attention deficits in adult ADHD. Journal of Attention Disorders, 11(6), 664-76. doi:10.1177/1087054707305108

Murphy, K., \& Barkley, R. A. (1996). Prevalence of DSM-IV symptoms of ADHD in adult licensed drivers: Implications for clinical diagnosis. Journal of Attention Disorders. doi:10.1177/108705479600100303

Nigg, J. T. (2005). Neuropsychologic theory and findings in attentiondeficit/hyperactivity disorder: the state of the field and salient challenges for the coming decade. Biological Psychiatry, 57(11), 1424-35. doi:10.1016/j.biopsych.2004.11.011

O'Connell, R. G., Bellgrove, M. a, Dockree, P. M., Lau, A., Fitzgerald, M., \& Robertson, I. H. (2008). Self-Alert Training: volitional modulation of autonomic arousal improves sustained attention. Neuropsychologia, 46(5), 1379-90. doi:10.1016/j.neuropsychologia.2007.12.018

Pietrzak, R. H., Mollica, C. M., Maruff, P., \& Snyder, P. J. (2006). Cognitive effects of immediate-release methylphenidate in children with attention-deficit/hyperactivity disorder. Neuroscience and Biobehavioral Reviews, 30(8), 1225-45. doi:10.1016/j.neubiorev.2006.10.002

Rheinberg, F., Vollmeyer, R., \& Burns, B. D. (2001). QCM : A questionnaire to assess current motivation in learning situations. Diagnostica, 47(2), 57-66. doi:10.1026//0012-1924.47.2.57

Robertson, I. H., Manly, T., Andrade, J., Baddeley, B. T., \& Yiend, J. (1997). "Oops!”: performance correlates of everyday attentional failures in traumatic brain injured and normal subjects. Neuropsychologia, 35(6), 747-58.

Sattler. (2001). Assessment of children: Cognitive applications (4th ed.). San Diego, CA, USA: Jerome M. Sattler Publishers, Inc.

Scheinost, D., Stoica, T., Saksa, J., Papademetris, X., Constable, R. T., Pittenger, C., \& Hampson, M. (2013). Orbitofrontal cortex neurofeedback produces lasting changes in contamination anxiety and resting-state connectivity. Translational Psychiatry, 3(4), e250. doi:10.1038/tp.2013.24

Schneider, M. F., Krick, C. M., Retz, W., Hengesch, G., Retz-Junginger, P., Reith, W., \& Rösler, M. (2010). Impairment of fronto-striatal and parietal cerebral networks correlates with attention deficit hyperactivity disorder (ADHD) psychopathology in adults - a functional magnetic resonance imaging (fMRI) study. Psychiatry Research, 183(1), 75-84. doi:10.1016/j.pscychresns.2010.04.005 
Scott, N. W., McPherson, G. C., Ramsay, C. R., \& Campbell, M. K. (2002). The method of minimization for allocation to clinical trials. a review. Controlled Clinical Trials, 23(6), 662-74.

Simon, V., Czobor, P., Bálint, S., Mészáros, A., \& Bitter, I. (2009). Prevalence and correlates of adult attention-deficit hyperactivity disorder: meta-analysis. The British Journal of Psychiatry: The Journal of Mental Science, 194(3), 204-211. doi:10.1192/bjp.bp.107.048827

Slaats-Willemse, D., Swaab-Barneveld, H., De Sonneville, L., \& Buitelaar, J. (2005). Familial clustering of executive functioning in affected sibling pair families with ADHD. Journal of the American Academy of Child and Adolescent Psychiatry, 44(4), 385-91. doi:10.1097/01.chi.0000153227.34473.c7

Smilek, D., Carriere, J. S. a, \& Cheyne, J. A. (2010). Failures of sustained attention in life, lab, and brain: ecological validity of the SART. Neuropsychologia, 48(9), 2564-70. doi:10.1016/j.neuropsychologia.2010.05.002

Sonuga-Barke, E. J. S., Brandeis, D., Cortese, S., Daley, D., Ferrin, M., Holtmann, M., ... Sergeant, J. (2013). Nonpharmacological interventions for ADHD: systematic review and meta-analyses of randomized controlled trials of dietary and psychological treatments. The American Journal of Psychiatry, 170(3), 275-89. doi:10.1176/appi.ajp.2012.12070991

Spencer, T. J., Biederman, J., \& Mick, E. (2007). Attention-deficit/hyperactivity disorder: diagnosis, lifespan, comorbidities, and neurobiology. Journal of Pediatric Psychology, 32(6), 631-42. doi:10.1093/jpepsy/jsm005

Subramanian, L., Hindle, J. V, Johnston, S., Roberts, M. V, Husain, M., Goebel, R., \& Linden, D. (2011). Real-time functional magnetic resonance imaging neurofeedback for treatment of Parkinson's disease. The Journal of Neuroscience: The Official Journal of the Society for Neuroscience, 31(45), 16309-17. doi:10.1523/JNEUROSCI.3498-11.2011

Swanson, J., Baler, R. D., \& Volkow, N. D. (2011). Understanding the effects of stimulant medications on cognition in individuals with attention-deficit hyperactivity disorder: a decade of progress. Neuropsychopharmacology: Official Publication of the American College of Neuropsychopharmacology, 36(1), 207-26. doi:10.1038/npp.2010.160

Talairach, J., \& Tournoux, P. (1988). Co-planar stereotaxic atlas of the human brain: 3-dimensional proportional system: an approach to cerebral imaging. Neuropsychologia (Vol. 39). New York: Thieme Medical Publishers.

Van de Loo-Neus, G. H. H., Rommelse, N., \& Buitelaar, J. K. (2011). To stop or not to stop? How long should medication treatment of attention-deficit hyperactivity disorder be extended? European Neuropsychopharmacology.

Van Dongen-Boomsma, M., Vollebregt, M. a, Slaats-Willemse, D., \& Buitelaar, J. K. (2013). A randomized placebo-controlled trial of electroencephalographic (EEG) neurofeedback in children with attention-deficit/hyperactivity disorder. The Journal of Clinical Psychiatry, 74(8), 821-7. doi:10.4088/JCP.12m08321 
Van Veen, M. M., Kooij, J. J. S., Boonstra, A. M., Gordijn, M. C. M., \& Van Someren, E. J. W. (2010). Delayed Circadian Rhythm in Adults with AttentionDeficit/Hyperactivity Disorder and Chronic Sleep-Onset Insomnia. Biological Psychiatry, 67(11), 1091-1096.

Vollebregt, M. a, van Dongen-Boomsma, M., Buitelaar, J. K., \& Slaats-Willemse, D. (2013). Does EEG-neurofeedback improve neurocognitive functioning in children with attention-deficit/hyperactivity disorder? A systematic review and a doubleblind placebo-controlled study. Journal of Child Psychology and Psychiatry, and Allied Disciplines. doi:10.1111/jcpp.12143

Wechsler, D. (2008). Wechsler Adult Intelligence Scale, Fourth Edition (WAIS-IV) (4th ed.). San Antonio, TX, USA: Pearson.

Weiskopf, N. (2012). Real-time fMRI and its application to neurofeedback. Neurolmage, 62(2), 682-92. doi:10.1016/j.neuroimage.2011.10.009

Willcutt, E. G., Doyle, A. E., Nigg, J. T., Faraone, S. V, \& Pennington, B. F. (2005). Validity of the executive function theory of attention-deficit/hyperactivity disorder: a meta-analytic review. Biological Psychiatry, 57(11), 1336-46. doi:10.1016/j.biopsych.2005.02.006

Young, K. D., Zotev, V., Phillips, R., Misaki, M., Yuan, H., Drevets, W. C., \& Bodurka, J. (2014). Real-time FMRI neurofeedback training of amygdala activity in patients with major depressive disorder. PloS One, 9(2), e88785. doi:10.1371/journal.pone.0088785 
180 | CHAPTER 5 
Summary and Conclusions 


\section{Summary of results}

In 2010, only two studies on potential clinical application of fMRI-based neurofeedback had been published (chapter 1), one on reducing pain symptoms by modulating activation levels in a brain regions involved in conscious pain perception by deCharms et al. (2005), and another on learning how to attenuate the response of hyper-activated auditory cortex in tinnitus patients (Haller, Birbaumer, \& Veit, 2010). Since 2010, publications on fMRI neurofeedback studies have grown rapidly (Sulzer et al., 2013), and several other pioneering studies in new patient groups, such as Parkinson and depressed patients have been concluded (Linden et al., 2012; Subramanian et al., 2011; Young et al., 2014).

Among recent methodological advancements in fMRI neurofeedback research, the development of indicators of functional brain connectivity for neurofeedback trainings was something that had long been proposed, but only recently been investigated (Koush et al., 2013; Weiskopf et al., 2004; Zilverstand, Sorger, Zimmermann, Kaas, \& Goebel, 2014). The presented study explored if fMRI-based functional connectivity neurofeedback indicators, derived from short-window time course data at the level of single task periods, would provide unique, and relevant information on ongoing brain processing (chapter 2). The results demonstrated that short-window functional brain connectivity measures indeed capture unique information on relevant task aspects, and may therefore be a valuable tool for fMRI neurofeedback trainings (chapter 2). As the general goal in clinical applications of neurofeedback must be optimizing the information content of the feedback signal, and functional connectivity measures are among the most important biomarkers of neuropsychiatric disease, this has important implications for designing future clinical neurofeedback trainings (chapter 2).

To ensure that the selection of the feedback target region in a subsequent neurofeedback training in spider phobia would be well grounded in clinical neuroscience research, a study on the representation of subjective anxiety levels in the brain was conducted (chapter 3). Increasing levels of anxiety were provoked using a newly created, and validated stimulus set, and a parametric analysis of the fMRI data was conducted to investigate which brain regions hold a quantitative representation of subjective anxiety levels (chapter 3 ). The results demonstrated that processing of anxiety-provoking stimuli was organized hierarchically, and that brain regions at a 
lower processing level were involved in initial threat monitoring, while regions at an intermediate level indeed represented subjective anxiety levels (chapter 3 ). The study therefore provided important knowledge regarding which regions may be used for indexing subjective anxiety in the planned neurofeedback training in spider phobia (chapter 4).

Next, a neurofeedback training for spider phobics was implemented, guiding the participants with intermittent neurofeedback while they were learning how to regulate anxiety (chapter 4). In a controlled, randomized study, the neurofeedback group received feedback from the left dorsolateral prefrontal cortex and the right insula, as an indicator of engagement and regulation success, while the control group completed the same training, but did not receive any feedback information. Results from the analysis of the subjective anxiety ratings, and brain activation levels, demonstrated that neurofeedback participants achieved better down-regulation of anxiety during the training than control subjects. It was concluded that the provided neurofeedback enhanced the efficacy of learning to regulate clinical levels of anxiety (chapter 4). The implemented neurofeedback training had a focus on the cognitively more demanding representational learning, and seemed to be well suited for the target group, which was recruited from spider phobic undergraduate students.

The fourth experimental study was conducted with adults with attentiondeficit/hyperactivity disorder with high initial levels of impairment of cognitive functioning (chapter 5). Participants attended four weekly fMRI neurofeedback training sessions, during which they learned to up-regulate pathologically reduced activation levels in anterior cingulate cortex by performing a mental calculation task at varying levels of difficulty, while receiving guidance from continuously provided neurofeedback information. The study was designed as an exploratory, randomized controlled treatment study with blinding of the participants. Prior, and after the training, ADHD symptoms and cognitive functioning were assessed by neuropsychological testing. Results showed that neurofeedback participants achieved a significant increase in ACC activation levels over sessions. They did not demonstrate a significant reduction of ADHD symptoms, but improved considerably and significantly on measures of attentional control and working memory. Individual modulation performance was highly correlated with ability to sustain attention and working memory capacity. However, modulation success, was not highly correlated with treatment change. It could therefore not be finally evaluated if the improvements were a direct result of the 
neurofeedback guided modulation of anterior cingulate cortex activation levels, or a non-specific effect of the training per se (chapter 5). It was noticeable, that the individual ability to modulate the signal was generally lower than in the previous study (chapter 4, 5), even though the implemented approach was designed to be less cognitively demanding, as procedures were optimized towards supporting reinforcement learning, rather than representational learning. It was nonetheless remarkable, that the implemented training had a large positive effects on cognitive functioning, also in participants with initial high impairments.

\section{Conclusions}

Research on fMRI neurofeedback trainings for clinical applications is still in its infancy. In the future, it will be important to keep improving online analysis methods, and expanding available tools. It is furthermore of equal significance to firmly ground implemented setups in clinical neuroscience research, and tailor learning paradigms towards the targeted patient group. On a personal note, the presented research was very rewarding, as all participants were highly motivated, and expressed a large desire to further improve treatments for mental health. If neurofeedback training setups could be further optimized, they may thus indeed provide a unique opportunity for a novel, and efficacious treatment method. Sometime in the future, we may indeed learn 'changing our brain states' in order to change our behavior. 


\section{References}

DeCharms, R. C., Maeda, F., Glover, G. H., Ludlow, D., Pauly, J. M., Soneji, D., ... Mackey, S. C. (2005). Control over brain activation and pain learned by using realtime functional MRI. Proceedings of the National Academy of Sciences of the United States of America, 102(51), 18626-31. doi:10.1073/pnas.0505210102

Haller, S., Birbaumer, N., \& Veit, R. (2010). Real-time fMRI feedback training may improve chronic tinnitus. European Radiology, 20(3), 696-703. doi:10.1007/s00330-009-1595-z

Koush, Y., Rosa, M. J., Robineau, F., Heinen, K., W Rieger, S., Weiskopf, N., ... Scharnowski, F. (2013). Connectivity-based neurofeedback: dynamic causal modeling for real-time fMRI. Neurolmage, 81, 422-30. doi:10.1016/j.neuroimage.2013.05.010

Linden, D. E. J., Habes, I., Johnston, S. J., Linden, S., Tatineni, R., Subramanian, L., ... Goebel, R. (2012). Real-time self-regulation of emotion networks in patients with depression. PloS One, 7(6), e38115. doi:10.1371/journal.pone.0038115

Subramanian, L., Hindle, J. V, Johnston, S., Roberts, M. V, Husain, M., Goebel, R., \& Linden, D. (2011). Real-time functional magnetic resonance imaging neurofeedback for treatment of Parkinson's disease. The Journal of Neuroscience: The Official Journal of the Society for Neuroscience, 31(45), 16309-17. doi:10.1523/JNEUROSCI.3498-11.2011

Sulzer, J., Haller, S., Scharnowski, F., Weiskopf, N., Birbaumer, N., Blefari, M. L., ... Sitaram, R. (2013). Real-time fMRI neurofeedback: progress and challenges. Neurolmage, 76, 386-99. doi:10.1016/j.neuroimage.2013.03.033

Weiskopf, N., Mathiak, K., Bock, S. W., Scharnowski, F., Veit, R., Grodd, W., ... Birbaumer, N. (2004). Principles of a brain-computer interface (BCl) based on real-time functional magnetic resonance imaging (fMRI). IEEE Transactions on Bio-Medical Engineering, 51(6), 966-70. doi:10.1109/TBME.2004.827063

Young, K. D., Zotev, V., Phillips, R., Misaki, M., Yuan, H., Drevets, W. C., \& Bodurka, J. (2014). Real-time FMRI neurofeedback training of amygdala activity in patients with major depressive disorder. Plos One, 9(2), e88785. doi:10.1371/journal.pone.0088785

Zilverstand, A., Sorger, B., Zimmermann, J., Kaas, A., \& Goebel, R. (2014). Windowed Correlation: A Suitable Tool for Providing Dynamic fMRI-Based Functional Connectivity Neurofeedback on Task Difficulty. PLOS ONE, 9(1), e85929. doi:10.1371/journal.pone.0085929 
186 | SUMMARY AND CONCLUSIONS 


\section{Acknowledgments}

First of all, many thanks to my supervisor Rainer Goebel - his never ending optimism was great complement to my own complicated worrisome thoughts, and there were many inspiring moments, which helped this work along, especially when things got difficult! Also, a great thank you to Bettina Sorger, for continuously making sure that everything was perfect to the last detail - this dedication still amazes me! My biggest thanks go to the most essential person to this work, Jan Zimmermann, who helped me in a million ways on a personal and professional level, and made this book happen. Also, many great thanks to all my friends for listening and helping when I got stuck! You know who you are, but the ones on who's doors I knocked most often were: Franziska Dambacher, who is the most supportive person I could ever imagine, Thomas Emmerling, an excellent listener who never failed to come up with creative ideas, Valentin Kemper, always a solid rock to trust, Katie Wheat, with who I enjoyed great discussions about all our possible and not so possible futures, and Judith Eck, who understood so well what I was talking about. Also, my deepest thanks to the Dücker-Lange-Meyer WG, who made sure that I actually started my PhD by treating me with philosophical kitchen discussions and tea times in our little "garden", and Andrea Wolf, my invaluable telephone joker! I also had many great times with Amanda Kaas, and Pegah Sarkheil, who were great office mates, collaborators, and friends! And with Rosanne Rademaker - many great chats, hope you do move to the US with us! Inge Timmers, thank you so much for always being so helpful! Claudia Bartz - I was very happy you came back! And, very important to actually finishing this thesis: my two fun weekend companions at Uns 40 and Oxfordlaan 55 (reliably there at the oddest hours), Michelle Moerel, and Anna Gardumi! Many thanks also to my friends who know nothing about neuroscience and took my mind of the topic once in a while: Maria Klamerski, Helena Stjerna, Irene Fuchs, and Tobias Breitenstein most of all... Finally, to two more people who were absolutely essential to the process: Bernadette Jansma, and Judith Vanhommerig, thanks for inspiring me and believing in me throughout these years! And, last, and certainly not least, many thanks to my family, Barbara Wolpers, Axel Schulz-Walz, and Franziska Schirrmacher, for playing a big part in me becoming me. 


\section{Curriculum Vitae}

Anna Zilverstand (née Schulz-Walz) was born on September 23 ${ }^{\text {rd }}$, 1975 in Leverkusen, Germany. In 1993 she graduated from Wynyard Composite High School in Wynyard, Saskatchewan, Canada. In Germany she received her High School Diploma from Freiherr-vom-Stein Schule in Leverkusen, Germany in 1995. In the same year she enrolled at the Faculty of Architecture at RWTH Aachen University in Germany to study Architecture and Urban Planning (Social Sciences), which she completed with the Diplom (B.Sc and M.Sc.) in 2001. The following three years she worked as a project and content manager for government funded sustainable energy projects in Cologne, Germany. In 2004 she enrolled at the University of Maastricht, in the Netherlands, at the Psychology Faculty. She completed the Bachelor of Psychology in 2007 with highest distinction. During her Bachelor studies she was a research assistant to Dr. Maaike Cima, and Prof. Bernadette Jansma at the Faculty of Psychology and Neuroscience. In 2009 she graduated with highest distinction from the Research Master of Cognitive Neuroscience, writing her thesis under the supervision of Prof. Rainer Goebel on the feasibility of fMRI-based neurofeedback in spider phobia. From 2009 on she continued this research, working as a PhD candidate with Prof. Rainer Goebel, and Dr. Bettina Sorger on the topic of clinical applications of fMRI-based neurofeedback. 


\section{Publications}

Peer reviewed journals:

Bolte, J., Jansma, B. M., Zilverstand, A., \& Zwitserlood, P. (2009). Derivational morphology approached with event-related potentials. The Mental Lexicon, 4(3), 336-353. doi:http://dx.doi.org/10.1075/ml.4.3.02bol

Goebel, R., Zilverstand, A., \& Sorger, B. (2010). Real-time fMRI-based braincomputer interfacing for neurofeedback therapy and compensation of lost motor functions. Imaging in Medicine, 2(4), 407-415. doi:10.2217/iim.10.35

Zilverstand, A., Sorger, B., Zimmermann, J., Kaas, A., \& Goebel, R. (2014). Windowed Correlation: A Suitable Tool for Providing Dynamic fMRI-Based Functional Connectivity Neurofeedback on Task Difficulty. PLOS ONE, 9(1), e85929. doi:10.1371/journal.pone.0085929

In preparation:

Zilverstand, A., Sorger, B., Sarkheil, P., Goebel, R.: fMRI neurofeedback enhances the efficacy of cognitive reappraisal for anxiety regulation in spider phobia.

Zilverstand, A., Kaemingk, A. Sorger, B., Goebel, R.: Measuring anxiety based on the $\mathrm{fMRI}$ BOLD signal. A parametric fMRI study in spider phobia.

Sarkheil, P., Zilverstand, A., Schneider, F. Goebel, R. Mathiak, K.: fMRI neurofeedback enhances emotion regulation as evidenced by a reduced amygdala response.

Cima, M., Zilverstand, A., Smulders, F.T.Y., Meijer, E.: Error monitoring in an offender sample.

\section{Poster Presentations:}

Zilverstand, A., Kaemingk, A., Sorger, B., Goebel, R. (2013). Towards therapy in the MRI scanner: Cognitive reappraisal of parametrically modulated anxiety in spider phobia. Poster presented at the 43rd Annual Meeting of the Society for Neuroscience in San Diego, California, USA.

Zilverstand, A., Sorger, B., Zimmermann, J., Goebel, R. (2012). Towards effective connectivity fMRI neurofeedback: single-block windowed partial correlations. Poster presented at the 18th Annual Meeting of the Organization for Human Brain Mapping in Beijing, China.

Zilverstand, A., Sorger, B., Zimmermann, J., Goebel, R. (2012). Developing connectivity measures for fMRI neurofeedback: single-block windowed 
correlation measures are modulated by finger tapping task. Poster presented at the 1st Swiss Real time fMRI Neurofeedback Conference, Zuerich, Switzerland.

Zilverstand, A., Sorger, B., Zimmermann, J., Sarkheil, P., Kaas, A., Goebel, R. (2011). Towards network fMRI neurofeedback: which information is encoded in activation level vs. correlation? Poster presented at the 17th Annual Meeting of the Organization for Human Brain Mapping in Quebec City, Canada.

Sarkheil, P., Zilverstand, A., Killian-Huetten, N., Schneider, F., Mathiak, K., Goebel, R. (2011). Application of Real-time fMRI-Neurofeedback in Treatment of Emotional Disorders. Poster presented at the 17th Annual Meeting of the Organization for Human Brain Mapping in Quebec City, Canada.

Sorger, B., Peters, J., van den Boomen, C., Zilverstand, A., Reithler, J., Goebel, R. (2010). Real-time decoding of the locus of visuospatial attention using multivoxel pattern classification. Poster presented at the 16th Annual Meeting of the Organization for Human Brain Mapping in Barcelona, Spain.

Talks:

Yale University, New Haven, USA: "Towards clinical applications in fMRI neurofeedback. Network approaches for learning how to change brain states", November 2013

Rutgers University, Newark, USA: "Towards therapy in the MRI scanner: Cognitive reappraisal of parametrically modulated anxiety in spider phobia", November 2013

Mount Sinai Hospital, NY, USA: "Towards clinical applications in fMRI neurofeedback. Network approaches for learning how to change brain states", November 2013

Child Mind Institute, NY, USA: "Towards clinical applications in fMRI neurofeedback. Learning how to change complex brain states", November 2013

BrainGain Consortium Meeting, NL: "Optimization of fMRI neurofeedback techniques and clinical applications of fMRI neurofeedback", March 2013

Training Dutch Medical Doctors, NL: "Anxiety and the Brain", February 2011

BrainGain Consortium Meeting, NL: "Clinical applications: fMRI neurofeedback for Therapy", October 2010 